\title{
Liquiditätszusammenhänge zwischen Kassa- und Derivatemärkten
}

\author{
Dissertation \\ zur Erlangung des wirtschaftswissenschaftlichen Doktorgrades \\ der Wirtschaftswissenschaftlichen Fakultät \\ der Georg-August-Universität Göttingen
}

vorgelegt von

Dipl.-Math. oec. Paolo Krischak

aus Marburg

Göttingen, 2016 
Erstgutachter: Prof. Dr. Olaf Korn

Zweitgutachter: Prof. Dr. Jan Muntermann

Drittgutachter: Prof. Dr. Stefan Dierkes

Tag der mündlichen Prüfung:

Gleichzeitig erschienen in (bei):

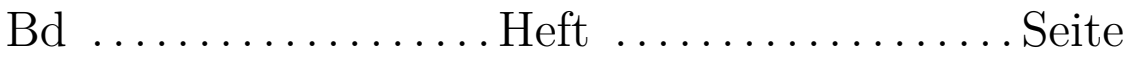

Ort .........................Jahr 


\section{Inhaltsverzeichnis}

$\begin{array}{lll}\text { Tabellenverzeichnis } & \text { III }\end{array}$

$\begin{array}{ll}\text { Abbildungsverzeichnis } & \text { IV }\end{array}$

1 Einleitung 1

2 Liquidität auf Finanzmärkten $\quad 6$

2.1 Liquiditätsbegriff . . . . . . . . . . . . . . . . . 6

2.2 Liquiditätsmessung . . . . . . . . . . . . . . . . . 7

2.2.1 Marktstrukturen .................. . 7

2.2.2 Round-Trip Kosten . . . . . . . . . . . . . . . . . . . . . . . 9 9

2.2 .3 Liquiditätsproxies . . . . . . . . . . . . . . . . . . . . 10

2.3 Determinanten von Liquidität . . . . . . . . . . . . . . . . . . . . . . 12

2.3.1 Inventarproblematik . . . . . . . . . . . . . . . . . 12

2.3 .2 Asymmetrische Information . . . . . . . . . . . . . . 16

2.3.3 Empirische Evidenz . . . . . . . . . . . . . . . . . . 19

2.4 Auswirkungen von Liquidität _. . . . . . . . . . . . . . . . 20

2.4.1 Preiseffekte . . . . . . . . . . . . . . . . . 20

2.4.1.1 Allgemeine Effekte auf Preise . . . . . . . . . . . 20

2.4.1.2 Effekte auf Futurespreise . . . . . . . . . . . . 21

2.4.1.3 Effekte auf Optionspreise . . . . . . . . . . . 22

2.4.2 Liquiditätseffekte . . . . . . . . . . . . . . . . . . . 24

2.4.2.1 Futuresmärkte . . . . . . . . . . . . 24

2.4 .2 .2 Optionsmärkte . . . . . . . . . . . . . 26

2.4.2.3 ETF-Märkte . . . . . . . . . . . . . 29

3 Liquiditäts- und Preiseffekte am Forwardmarkt 30

3.1 Einperiodenmodell . . . . . . . . . . . . . . . . . . . . . . . 30

3.1 .1 Basisfall . . . . . . . . . . . . . . . . . . 31

3.1.2 Geld-Brief-Spanne am Kassamarkt . . . . . . . . . . . . . . . . . 40

3.1.3 Asymmetrische Preiseinflussfunktion und Leerverkaufsverbote . . 43

3.1.4 Liquiditätsrisiko am Kassamarkt . . . . . . . . . . . . . . . . . . 46

3.2 Mehrperiodenmodell . . . . . . . . . . . . . . . . 52

3.2.1 Modellstruktur . . . . . . . . . . . . . . . . 52 
3.2.2 Kassamarktpositionen des Market Makers . . . . . . . . . . . . . 54

3.2.3 Preiseffekte . . . . . . . . . . . . . . . . . . . 57

3.2.4 Liquiditätseffekte . . . . . . . . . . . . . . . . . . 60

4 Empirische Analyse der Preis- und Liquiditätseffekte 62

4.1 Untersuchte Aktien- und Futuresmärkte . . . . . . . . . . . . . . . . 62

4.2 Preiseffekte . . . . . . . . . . . . . . . . . . 65

4.2.1 Variablen und erwartete Effekte . . . . . . . . . . . . . 65

4.2.2 Deskriptive Statistik . . . . . . . . . . . . . . . . . . . 68

4.2.3 Determinanten von Futurespreisen . . . . . . . . . . . . . . 72

4.2.3.1 Basisfall . . . . . . . . . . . . . . . 73

4.2.3.2 Alternative Risikomaße . . . . . . . . . . . . . . 75

4.2.3.3 Dividendeneffekte . . . . . . . . . . . . . . 77

4.2.3.4 Effekte von Finanzierungskosten . . . . . . . . . . . 80

4.2.3.5 Futuresliquidität . . . . . . . . . . . . . 82

4.2.3.6 Simultane Effekte . . . . . . . . . . . . . 82

4.3 Liquiditätseffekte . . . . . . . . . . . . . . . . . . 85

4.3.1 Variablen und erwartete Effekte . . . . . . . . . . . . 85

4.3 .2 Deskriptive Statistik . . . . . . . . . . . . . . . . 88

4.3.3 Determinanten der Futuresliquidität . . . . . . . . . . . . . 96

4.3.3.1 Basisfall . . . . . . . . . . . . . . . . . 96

4.3.3.2 Alternative Risikomaße . . . . . . . . . . . . . . . . . 98

4.3.3.3 Dividendeneffekte . . . . . . . . . . . . . . . . 100

4.3.3.4 Effekte von Finanzierungskosten . . . . . . . . . . . . . 101

4.3.3.5 Resultate für Indexfutures . . . . . . . . . . . . . . . . . 104

5 Schlussbetrachtung und Ausblick 108

$\begin{array}{ll}\text { Literaturverzeichnis } & 112\end{array}$

$\begin{array}{lr}\text { A Anhang } & 120\end{array}$

A.1 Liquiditätsrisiko am Kassamarkt: Herleitung des Forwardpreises und der optimalen Kassamarktposition des Market Makers . . . . . . . . . . . . . 120

A.2 Simultane Liquiditätseffekte . . . . . . . . . . . . . . . . . . . . 122 


\section{Tabellenverzeichnis}

4.1 Absolutbetrag der relativen Abweichungen der SSF-Preise von den Costof-Carry-Preisen . . . . . . . . . . . . . . . . . . . . 70

4.2 Illiquidität und Volatilität der Aktien . . . . . . . . . . . . . . . . . 71

4.3 Determinanten der Abweichungen von den Cost-of-Carry-Preisen: Basisfall 74

4.4 Determinanten der Abweichungen von den Cost-of-Carry-Preisen: Alternative Risikomaße . . . . . . . . . . . . . . . . . . . . . . . . . . . . 76

4.5 Dividendenbesteuerung . . . . . . . . . . . . . . . . 78

4.6 SSF Handelsvolumen $(01.01 .2010$ - 01.04.2013) . . . . . . . . . . . . . 78

4.7 Determinanten der Abweichungen von den Cost-of-Carry-Preisen: Dividenden . . . . . . . . . . . . . . . . . . . . . . 79

4.8 Determinanten der Abweichungen von den Cost-of-Carry-Preisen: Finanzierungskosten . . . . . . . . . . . . . . . . . . 81

4.9 Determinanten der Abweichungen von den Cost-of-Carry-Preisen: Futuresilliquidität . . . . . . . . . . . . . . . 83

4.10 Determinanten der Abweichungen von den Cost-of-Carry-Preisen: Simultane Effekte . . . . . . . . . . . . . . . . . . . . . . . 84

4.11 Illiquiditätsmaße der Aktien . . . . . . . . . . . . . . . . . . . . . 89

4.12 Illiquiditätsmaße der kurzfristigen SSF . . . . . . . . . . . . . . . . . 90

4.13 Illiquiditätsmaße der langfristigen SSF . . . . . . . . . . . . . . . . . . . 92

4.14 Volatilität und Liquiditätsrisiko der Aktien . . . . . . . . . . . . . . . . 93

4.15 Geld-Brief-Spanne und Liquiditätsrisiko der Aktien . . . . . . . . . . . . 94

4.16 Geld-Brief-Spanne der SSF . . . . . . . . . . . . . . . . . . 95

4.17 Determinanten der Futuresliquidität: Basisfall. . . . . . . . . . . . . . . 97

4.18 Determinanten der Futuresliquidität: Effekte alternativer Risikomaße . 99

4.19 SSF Handelsvolumen (01.01.2010 - 31.12.2011) . . . . . . . . . . . . . . . 101

4.20 Determinanten der Futuresliquidität: Dividenden . . . . . . . . . . . . 102

4.21 Determinanten der Futuresliquidität: Finanzierungsliquidität . . . . . . . 103

4.22 Illiquiditätsmaße der Indexfutures . . . . . . . . . . . . . . . . . 105

4.23 Determinanten der Futuresliquidität: Indexfutures . . . . . . . . . . . . 107

A.1 Determinanten der Futuresliquidität: Simultane Effekte . . . . . . . . . . 122 


\section{Abbildungsverzeichnis}

3.1 Orderbücher Kassa- und Forwardmarkt: Basisfall . . . . . . . . . . . . . 38

3.2 Orderbücher Kassa- und Forwardmarkt: Geld-Brief-Spanne . . . . . . . . 42

3.3 Orderbücher Kassa- und Forwardmarkt: Asymmetrische Preiseinflussfunk-

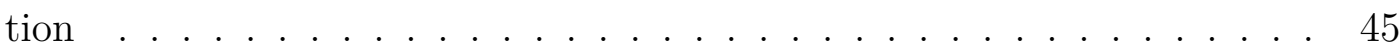

3.4 Orderbücher Kassa- und Forwardmarkt: Leerverkaufsverbote . . . . . . . 46

3.5 Illiquidität am Forwardmarkt: Effekte von Kassamarktliquidität, Risikoaversion, Kassamarktvolatilität und Liquiditätsrisiko . . . . . . . . . . 49

3.6 Illiquidität am Forwardmarkt: Effekte der Korrelation von Preis- und Liquiditätsrisiko sowie der erwarteten Rendite . . . . . . . . . . . . . . . 50

4.1 Relative Abweichungen der SSF-Preise von den Cost-of-Carry-Preisen . . 69 


\section{Einleitung}

Die klassische Bewertungstheorie von Derivaten fußt auf der Annahme friktionsloser Märkte, womit u. a. unterstellt wird, dass sowohl das Basisinstrument als auch die darauf geschriebenen Derivate perfekt liquide sind. In neueren Studien wird diese Annahme teilweise aufgehoben. So modellieren Cetin et al. (2006) und Liu \& Yong (2005) die Illiquidität am Kassamarkt und analysieren die Auswirkungen auf Optionspreise. Die Autoren untersuchen jedoch nicht explizit die Liquiditätseffekte am Derivatemarkt. Grundsätzlich ist davon auszugehen, dass sowohl Kassa- als auch Derivatemarkt nicht perfekt liquide sind und die Liquidität auf beiden Märkten in einer engen Verbindung steht. Eine Analyse der möglichen Liquiditätszusammenhänge von Kassa- und Derivatemärkten erfordert folglich die gemeinsame Betrachtung der Liquidität beider Märkte.

Die Fragestellung, wie die Liquidität von Kassa- und Derivatemärkten zusammenhängt, ist insbesondere vor dem Hintergrund von Übertragungseffekten zwischen Märkten, wie sie während der Finanzkrise aufgetreten sind, von Bedeutung. Regulatoren müssen die Liquiditätszusammenhänge verstehen, um die Auswirkungen ihrer Maßnahmen wie z. B. das Verbot von Leerverkäufen beurteilen zu können. Die Thematik ist darüber hinaus für Investoren und die Betreiber von Wertpapierbörsen von Relevanz. Investoren streben es an, die Illiquiditätskosten ihrer Handelsstrategien zu minimieren, und die Betreiber von Wertpapierbörsen versuchen, die Attraktivität ihres Handelsplatzes durch eine hohe Liquidität der dort gehandelten Wertpapiere zu erhöhen.

In welcher Beziehung die Liquidität auf Kassa- und Derivatemärkten zueinander steht, ist nicht eindeutig. Abhängig davon, ob auf den Märkten Substituts- oder Komplementärgüter gehandelt werden, sind zwei unterschiedliche Sichtweisen denkbar. Ist Ersteres der Fall, könnte sich eine inverse Liquiditätsbeziehung ergeben, was als Substitutionshypothese bezeichnet wird. Folgende Argumentation könnte dieser Sichtweise zugrundeliegen: Kommt es zu einem exogenen Schock, der die Liquidität nur eines Marktes ansteigen lässt, könnten Investoren auf diesen Markt wechseln. Wechseln uninformierte Investoren den Markt, würde dies dazu führen, dass sich die Informationsasymmetrien und damit die Liquidität auf beiden Märkten in unterschiedliche Richtungen entwickeln. Subrahmanyam (1991) analysiert einen solchen Wechsel vom Kassa- auf den Indexfuturesmarkt modelltheoretisch. Er argumentiert, dass die Einführung von Indexfutures die Liquidität des Kassamarktes verringert, da uninformierte Investoren aufgrund höherer 
Kosten adverser Selektion am Kassamarkt auf den Markt für Indexfutures wechseln. In ihrer empirischen Analyse untersuchen Jegadeesh \& Subrahmanyam (1993) die Auswirkungen der Einführung von S\&P 500 Indexfutures und finden Evidenz für eine inverse Liquiditätsbeziehung von Aktien- und Indexfuturesmarkt.

Werden Kassa- und Derivatemärkte als Komplementäre angesehen, kann eine gleichgerichtete Liquiditätsbeziehung durch die Hedgingaktivitäten von Market Makern erklärt werden. In ihrer grundlegenden Studie entwickeln Cho \& Engle (1999) die sogenannte „derivative-hedge-theory“ für Optionsmärkte. Ihre Theorie basiert auf der Annahme, dass die Market Maker am Optionsmarkt ihre Positionen am Kassamarkt absichern. Erhöht sich die Illiquidität am Kassamarkt, hat dies einen Anstieg der Hedgingkosten der Market Maker zur Folge. Um für diese zusätzlichen Kosten entschädigt zu werden, werden die Market Maker eine größere Geld-Brief-Spanne am Optionsmarkt stellen und damit eine gleichgerichtete Bewegung der Liquidität auf beiden Märkten bewirken. Unterschiedliche Studien zu Optionsmärkten finden empirische Evidenz für eine positive Liquiditätsbeziehung zwischen Aktien- und Optionsmärkten und unterstützen damit die komplementäre Sichtweise der beiden Märkte (vgl. z. B. Kaul et al. (2004), Engle \& Neri (2010) und Goyenko et al. (2014)).

Die zitierten Studien zur Liquiditätsbeziehung von Aktien- und Optionsmärkten behandeln die Zusammenhänge etablierter Märkte. Frühere Studien untersuchen außerdem die Auswirkungen der Einführung von neuen Optionsmärkten und finden ebenfalls Evidenz für eine gleichgerichtete Liquiditätsbeziehung (vgl. z. B. Damodaran \& Lim (1991), Fedenia \& Grammatikos (1992) und Kumar et al. (1998)). Über die Liquiditätszusammenhänge von Aktien- und Futuresmärkten ist dagegen wenig bekannt. Bisher beschränkt sich die Forschung auf die Untersuchung der Auswirkungen der Einführung neuer Indexfuturesmärkte (Subrahmanyam (1991) und Jegadeesh \& Subrahmanyam (1993)). Es existieren weder theoretische noch empirische Studien, die die Liquiditätszusammenhänge etablierter Aktien- und Futuresmärkte analysieren. Zentrales Ziel der vorliegenden Arbeit ist es, diese Forschungslücke zu schließen, indem zum ersten Mal die „derivativehedge-theorie“ für Futures explizit modelliert und damit eine theoretische Basis zur Analyse der Liquiditätszusammenhänge geschaffen wird. Aufbauend auf der theoretischen Untersuchung wird zusätzlich anhand einer umfangreichen empirischen Analyse ein besseres Verständnis der Liquiditätszusammenhänge von Aktien- und Futuresmärkten gewonnen.

Der erste Beitrag dieser Arbeit besteht in der Entwicklung eines Modells in Anleh- 
nung an Gârleanu et al. (2009), in dem die Angebotsseite auf dem Futuresmarkt durch einen repräsentativen Market Maker beschrieben wird, welcher die Nachfrage bedient und die Möglichkeit besitzt, seine Futuresposition über den Kassamarkt abzusichern. Die entscheidende Erweiterung ist, dass der Kassamarkt nicht perfekt liquide ist. Im Rahmen dieses Modells kann eine Bewertungsgleichung für Futures in Abhängigkeit der Nachfrage abgeleitet werden. Dieser Zusammenhang ermöglicht es, die Auswirkungen der Liquidität am Kassamarkt auf die Liquidität kurz- und langfristiger Kontrakte am Futuresmarkt zu analysieren. Anhand verschiedener Modellvarianten wird untersucht, wie diese Effekte durch die Berücksichtigung von unterschiedlichen Liquiditätsdimensionen, von Leerverkaufsbeschränkungen, eines Liquiditätsrisikos am Kassamarkt sowie der Risikopräferenzen des Market Makers und dessen Möglichkeit, sich dynamisch am Kassamarkt abzusichern, beeinflusst werden. Die Modellvariante mit Liquiditätsrisiko wird ebenfalls in der Studie von Korn et al. (2015) analysiert. Aus der modelltheoretischen Analyse werden empirisch testbare Hypothesen zur Liquiditätsübertragung vom Kassaauf den Futuresmarkt abgeleitet. Insbesondere zeigt sich, dass sich die Liquidität nicht eins zu eins überträgt, sondern mit anderen Faktoren wie dem Preis- und Liquiditätsrisiko am Kassamarkt sowie der Risikoaversion des Market Makers interagiert.

Ein weiterer wichtiger Aspekt ist, dass die Liquidität am Kassamarkt nicht nur Auswirkungen auf die Liquidität, sondern auch auf die No-Arbitrage-Preise am Futuresmarkt haben sollte. Im Rahmen eines Modells finden De Roon et al. (2000) allgemeine Evidenz, dass die Nachfrage nach Futures als Hedginginstrument bei Marktfriktionen Auswirkungen auf Futurespreise hat. Die aus dem nachfragebasierten Modell dieser Arbeit abgeleitete Darstellung des theoretischen Midquotes ermöglicht es, im Unterschied zu De Roon et al. (2000) zu untersuchen, wie sich die spezielle Friktion der Kassamarktilliquidität auf den No-Arbitrage-Preis am Futuresmarkt auswirkt. Zu den erwarteten Preiseffekten werden ebenfalls empirisch testbare Hypothesen abgeleitet. Darüber hinaus beschreibt das Modell einen möglichen Kausalzusammenhang zwischen der Illiquidität am Kassamarkt und den Preisabweichungen der Futurespreise von den Cost-of-Carry-Preisen. Ohne eine theoretische Basis ist dieser Zusammenhang nicht eindeutig, da einerseits eine hohe Liquidität das Ausnutzen von Preisabweichungen erleichtert, aber andererseits Preisabweichungen Arbitrageure anziehen, die wiederum die Liquidität beeinflussen könnten (Roll et al. (2007)).

Der zweite Beitrag dieser Arbeit liegt in der empirischen Analyse der abgeleiteten Preishypothesen. Die bisherigen empirischen Studien zur Auswirkung von Marktfriktionen auf 
die Abweichung vom No-Arbitrage-Preis am Futuresmarkt konzentrieren sich insbesondere auf die Analyse von Indexfuturesdaten. Unter Verwendung von Indexfutures weist die Studie von Roll et al. (2007) einen positiven Zusammenhang zwischen der Illiquidität am Kassamarkt und den Preisabweichungen am Futuresmarkt nach. Die vorliegende Arbeit untersucht dagegen die Preisabweichungen am Markt für Single Stock Futures (SSF). Zur Überprüfung der Hypothesen sind SSF das ideale Instrument, da das Hedging eines SSF mit einem einzigen Instrument, dem zugrundeliegenden Basiswertpapier, erfolgen kann und damit die Struktur des Modells widerspiegelt. In der empirischen Analyse wird unter Verwendung verschiedener Liquiditätsmaße der Einfluss der Aktienilliquidität der Unternehmen des deutschen Aktienindex (DAX30) auf die Preisabweichungen der entsprechenden SSF untersucht. Darüber hinaus werden weitere aus dem Modell identifizierte Einflussfaktoren in die Analyse einbezogen. Die Robustheit der Ergebnisse wird gewährleistet, indem Kontrollvariablen für die Besonderheiten des deutschen SSF-Marktes sowie nicht im Modell abgebildete Friktionen aufgenommen werden.

Der dritte Beitrag dieser Arbeit besteht in der empirischen Analyse der abgeleiteten Liquiditätshypothesen, die ebenfalls in der Studie von Korn et al. (2015) untersucht werden. Die Analyse der Liquiditätsbeziehung von Kassa- und Futuresmärkten ist insbesondere von Bedeutung, da sich die empirische Evidenz der Optionsliteratur aus zwei Gründen nicht auf Futuresmärkte übertragen lässt. Erstens sind Aktien und Futures beides lineare Instrumente, was sie eher zu Substituten als Aktien und Optionen machen sollte. Zweitens ist das Hedgingargument für Futures weniger bedeutend als für Optionen, da zur Absicherung gegenüber Risiken in Futurespositionen keine dynamische Hedgestrategie benötigt wird. Zur Analyse der Liquiditätsbeziehungen werden in dieser Arbeit die im DAX30 gelisteten Aktien und die darauf gehandelten SSF sowie der entsprechende Indexfutures verwendet. Die Arbeit ist damit die erste, die die Liquiditätsbeziehungen von Aktien- und SSF-Markt untersucht. Die anschließende Analyse von Indexfutures ist insbesondere von Interesse, da das informationsbasierte Argument von Subrahmanyam (1991) für eine gleichgerichtete Liquiditätsbeziehung im Fall von Indexfutures eine noch größere Bedeutung haben sollte.

Die Arbeit hebt sich außerdem von anderen Studien zur Liquiditätsübertragung durch die Messung der Liquidität ab. In den bisherigen Studien zu Liquiditätszusammenhängen wurde die Liquidität überwiegend durch die Geld-Brief-Spanne gemessen. Im Rahmen des Modells kann jedoch gezeigt werden, dass sich bestimmte Nachfrageeffekte erst bei größeren Mengen beobachten lassen. Zur Überprüfung dieser Effekte ist es daher nötig 
bei der Messung der Liquidität die Kauf- und Verkaufsaufträge über den besten Geldund Briefkurs hinaus zu berücksichtigen. Diese Arbeit verwendet das von der deutschen Börse entwickelte Xetra Liquidity Measure (XLM), welches sämtliche Orderbuchinformationen in einer Kennzahl verdichtet und damit eine adäquate Messung der Liquidität gewährleistet.

Die Arbeit ist in fünf Kapitel gegliedert. Kapitel 2 widmet sich den Grundlagen dieser Arbeit. Dazu wird zunächst der Begriff Liquidität definiert, die Messung erläutert sowie mögliche Ursachen und Auswirkungen auf andere Finanzmärkte diskutiert. Das Modell zur Analyse der Liquiditäts- und Preiseffekte am Futuresmarkt wird in Kapitel 3 entwickelt. Darüber hinaus werden empirisch testbare Hypothesen zu den Preis- und Liquiditätseffekten abgeleitet. Kapitel 4 hat die empirische Analyse dieser Hypothesen zum Gegenstand. Es werden die Datenbasis vorgestellt, die erwarteten Effekte diskutiert und die Ergebnisse erklärt. Die Arbeit schließt mit einer Schlussbetrachtung und einem Ausblick auf mögliche weitere Forschung in Kapitel 5. 


\section{Liquidität auf Finanzmärkten}

Ziel dieses Kapitels ist es, in die Thematik der Liquidität auf Finanzmärkten einzuführen. Zunächst wird der Begriff Liquidität definiert und Konzepte sowie Problematiken der Liquiditätsmessung erläutert. Im Anschluss daran werden mögliche Erklärungen der Liquidität von Wertpapieren diskutiert und die Arbeit in den Rahmen der bestehenden Literatur zur Liquidität auf Terminmärkten eingeordnet.

\subsection{Liquiditätsbegriff}

Zu Beginn soll der im Rahmen dieser Arbeit verwendete Liquiditätsbegriff definiert werden. Die Liquidität eines Wertpapiers setzt sich aus verschiedenen Dimensionen zusammen (vgl. z. B. Kyle (1985), Hasbrouck \& Schwartz (1988) und Amihud \& Mendelson (1991)). Eine Definition, welche die wesentlichen Liquiditätsdimensionen aus der Literatur berücksichtigt und in dieser Arbeit verwendet wird, ist die Folgende:

Ein Wertpapier ist liquide, sofern es jederzeit in kleinen und großen Mengen ohne einen Aufschlag oder Abschlag auf den aktuellen Marktpreis gekauft oder verkauft werden kann.

Diese Definition umfasst drei Dimensionen, welche unterschiedliche Aspekte der Liquidität abdecken. Kyle (1985) bezeichnet diese Dimensionen als Enge („tightness“), Tiefe („depth“) und Erneuerungskraft („,resiliency“). ${ }^{1}$ Die erste Dimension erfasst die Kosten für die erste Einheit eines Wertpapiers. Ein Wertpapier wird als „tight“ bezeichnet, sofern die Differenz zwischen dem besten Kauf- und Verkaufskurs klein ist. Die zweite Dimension erfasst neben diesem Kosten- auch einen Mengenaspekt. Ein Wertpapier weist eine hohe Tiefe auf, sofern eine große Anzahl von Kauf- und Verkaufsaufträgen nahe dem aktuellen Marktpreis existieren. Stehen in einem flachen Markt nicht ausreichend Kauf- oder Verkaufsaufträge nahe dem Marktpreis zur Verfügung, um eine bestimmte Nachfrage zu bedienen, muss auf Aufträge welche weiter vom Marktpreis entfernt sind

\footnotetext{
${ }^{1}$ Insbesondere die Bezeichnungen der Liquiditätsdimensionen Enge und Tiefe sind in der Literatur nicht einheitlich. Hasbrouck \& Schwartz (1988) verwenden die Begriffe Tiefe („depth“) und Breite („breadth“), wobei sie mit Tiefe die Enge-Dimension von Kyle (1985) und mit Breite dessen TiefeDimension bezeichnen. In dieser Arbeit wird die Terminologie von Kyle (1985) verwendet.
} 
zurückgegriffen werden. Der damit verbundene Preisauf- bzw. Preisabschlag führt zu höheren Kosten für den Käufer bzw. Verkäufer. Die dritte Dimension umfasst den Aspekt, dass ein Wertpapier jederzeit ohne Abschlag (Aufschlag) verkauft (gekauft) werden kann. Die Erneuerungskraft gibt die Geschwindigkeit an, mit der die Liquidität nach einem Liquiditätsschock zu einem "normalen" Level zurückkehrt (Foucault et al. (2005)). Ein Liquiditätsschock bezeichnet dabei Auftragsungleichgewichte, welche z. B. durch große Aufträge entstehen und zu einer temporären Abweichung vom Fundamentalwert führen. Die Erneuerungskraft eines Wertpapiers ist umso höher, je schneller neue Aufträge eingestellt werden und sich der Preis wieder dem fundamentalen Wert annähert.

\subsection{Liquiditätsmessung}

Die Messung von Liquidität hängt insbesondere von den verfügbaren Daten ab. Je nach Art der Organisationsstruktur eines Marktes stehen unterschiedliche Daten und damit unterschiedliche Informationen zur Verfügung. Im ersten Teil dieses Abschnitts werden daher die gängigen Marktstrukturen und deren Informationsgehalt beschrieben. Im zweiten Teil dieses Abschnitts werden die Aspekte eines theoretisch optimalen Liquiditätsmaßes für Wertpapiere diskutiert. Im Zuge dessen wird auf die Schwierigkeiten eingegangen, alle Liquiditätsdimensionen eines Wertpapiers in einer einzigen Kennzahl zu erfassen. Im dritten Teil werden wichtige in der Literatur verwendete Maße, die die Liquidität eines Wertpapiers approximieren, vorgestellt.

\subsubsection{Marktstrukturen}

Die vorherrschenden Marktstrukturen lassen sich in drei Arten unterteilen. Es gibt erstens Märkte, bei denen die Investoren über Intermediäre agieren, zweitens Märkte, bei denen Investoren direkt interagieren, und drittens Mischformen dieser beiden Märkte. Sofern nicht anders angegeben, basieren die Beschreibungen der verschiedenen Marktstrukturen auf den Ausführungen von De Jong \& Rindi (2009) sowie Hasbrouck (2006).

Die erste Art der Marktstruktur wird als Dealermarkt bezeichnet. In diesen Märkten wird die Liquidität von sogenannten designierten Liquiditätsanbietern, welche auch als Market Maker bezeichnet werden, garantiert. Der Handel in einem Dealermarkt muss über die Market Maker erfolgen. Diese sind verpflichtet Geld- und Briefkurse zu stellen 
zu denen sie bereit sind die Kauf- und Verkaufsaufträge der Investoren zu bedienen und handeln dabei auf eigene Rechnung.

Märkte auf denen die Zusammenführung von Kauf- und Verkaufsaufträgen der Investoren direkt und ohne Intermediäre erfolgt werden als Ordermärkte bezeichnet. Bei dieser Art von Marktstruktur wird die Liquidität durch das Einstellen neuer Aufträge der Investoren garantiert. Die Preise und Mengen unausgeführter Aufträge werden in einem Orderbuch gelistet. Ein neuer Auftrag wird mit denen im Orderbuch bestehenden verglichen. Sofern die Preise übereinstimmen, wird der Handel zum Preis des zuerst eingestellten Auftrags vollzogen. Der Informationsgehalt eines Dealer- und Ordermarktes unterscheidet sich folglich dahingehend, dass in einem Ordermarkt nicht nur Informationen über die quotierten Geld- und Briefkurse vorliegen, sondern darüber hinaus Quotierungen für unterschiedliche Mengen existieren.

Die Aufträge selbst lassen sich in zwei Arten unterteilen, die sich unterschiedlich auf die Liquidität auswirken. Im Fall einer Limit-Order wird ein akzeptabler Preis und eine Menge spezifiziert, sowie ob es sich um einen Kauf oder Verkauf handelt. Diese Art von Auftrag wird nur zum spezifizierten Preis ausgeführt. Eine Limit-Order ist damit für Investoren geeignet, die sensitiv bezüglich des Preises und weniger an der unmittelbaren Ausführung der Order interessiert sind, da diese Art Auftrag das Risiko der Nichtausführung birgt. Ist ein Investor dagegen an der unmittelbaren Ausführung seines Auftrags interessiert und bereit einen weniger vorteilhaften Preis zu akzeptieren, hat er die Möglichkeit, eine Market-Order zu platzieren. Im Gegensatz zur Limit-Order wird kein Preis angegeben und die Order sofort ausgeführt, sofern Nachfrage oder Angebot ausreichend vorhanden sind. Market-Orders entziehen Liquidität, wohingegen Limit-Orders die Liquidität erhöhen, indem sie die Tiefe des Orderbuches vergrößern.

Als drittes sind Hybridmärkte zu nennen, welche die Struktur der beiden zuvor beschriebenen Märkte kombinieren. In solchen Märkten existiert ein Orderbuch und der Handel erfolgt analog zum Handel in einem Ordermarkt. Ist das Orderbuch leer fällt der Handelsmechanismus auf einen Dealermarkt zurück. In diesem Fall nehmen designierte Market Maker die Gegenposition zu den neu eintreffenden Aufträgen ein und gewährleisten damit deren unmittelbare Ausführung. In einem Hybridmarkt wird die Liquidität folglich nicht nur durch die von den Investoren übermittelten Aufträge bereitgestellt, sondern darüber hinaus durch designierte Market Maker garantiert. Der Informationsgehalt ist vergleichbar mit dem eines Ordermarktes mit dem Unterschied, dass in einem Hybridmarkt auch im Fall eines leeren Orderbuches Geld- und Briefkurse durch die Mar- 
ket Maker gestellt werden. Aktuell ist der Handel an den meisten Börsen in hybrider Form organisiert. Ein Beispiel ist der New York Stock Exchange (NYSE), der Spezialisten beschäftigt, die das Orderbuch verwalten und darüber hinaus als Market Maker agieren.

\subsubsection{Round-Trip Kosten}

In der Forschung zur Liquiditätsmessung existiert eine Vielzahl von Maßen, welche die Liquidität eines Wertpapiers anhand von unterschiedlichen Informationen quantifizieren. Je mehr Liquiditätsdimensionen durch ein Maß erfasst werden sollen, umso mehr Informationen werden benötigt. Im Folgenden soll das Konzept eines theoretisch optimalen Liquiditätsmaßes erläutert werden. Dieses Maß ermöglicht es alle in der Liquiditätsdefinition dieser Arbeit genannten Dimensionen zu erfassen und wird als „Cost-of-a-RoundTrip" (CRT) bezeichnet.

Der $\operatorname{CRT}(X)$ wird für eine Menge von $X$ Wertpapieren berechnet und gibt die Kosten eines simultanen Kaufs und Verkaufs dieser Menge an. Gemessen werden die Kosten in Prozent des Wertpapierkurses. In einem perfekt liquiden Markt sollte eine solche Transaktion keine Kosten verursachen. Irvine et al. (2000) messen anhand der Kosten dieser theoretischen Transaktion die Liquidität eines Wertpapiers, wobei kleinere Kosten für eine bestimmte Menge an Wertpapieren für eine größere Liquidität stehen. Durch den Parameter $X$ können die zwei Liquiditätsdimensionen Enge und Tiefe erfasst werden.

Ein Spezialfall des CRT $(X)$ ist die Geld-Brief-Spanne. Die Geld-Brief-Spanne, definiert als Differenz zwischen dem besten Kauf- und Verkaufskurs, ist unmittelbar mit den Kosten einer sofortigen Nachfrage verbunden und daher ein natürlicher Startpunkt, um die Kosten der Liquidität für ein Wertpapier zu messen (Amihud \& Mendelson (1986)). Wird der $\operatorname{CRT}(X)$ für eine Einheit berechnet, entspricht er der Geld-Brief-Spanne. Folglich kann die Dimension Enge zu einem bestimmten Zeitpunkt durch den CRT(1) adäquat erfasst werden.

In dieser Arbeit wird die Geld-Brief-Spanne auf Basis der quotierten Preise gemessen. Eine Alternative zur quotierten Geld-Brief-Spanne ist es die realisierte Geld-Brief-Spanne aus den Transaktionspreisen zu bestimmen. Die Geld-Brief-Spanne allein als Liquiditätsmaß zu verwenden, ist jedoch kritisch zu sehen, da diese nur für die Menge an Wertpapieren gültig ist, welche zum besten Geld- und Briefkurs angeboten werden. Bei 
Aufträgen, die über diese Menge hinausgehen, verliert das Maß an Bedeutung. Nach Jones \& Lipson (2001) kann ein Markt liquide bei kleinen Aufträgen sein und dennoch können die Preise sehr sensitiv auf große Aufträge reagieren.

Das Konzept des CRT $(X)$ ermöglicht es, für einen Ordermarkt zusätzlich die Dimension der Markttiefe zu erfassen, indem er nicht für eine Einheit sondern für eine bestimmte Menge $X$ an Wertpapieren berechnet wird. Übersteigt diese Menge $X$ die Menge an Wertpapieren, welche zum besten Geld- und Briefkurs verfügbar sind, werden die nächstbesten Angebote aus dem Orderbuch berücksichtigt, um die Gesamtkosten der theoretischen Transaktion zu berechnen. ${ }^{2}$ Durch das Abbilden sämtlicher Orderbuchinformationen in einer Kennzahl kann die Dimension Tiefe zu einem bestimmten Zeitpunkt anhand des $\operatorname{CRT}(X)$ angemessen quantifiziert werden.

Neben der Erfassung der Liquiditätsdimensionen Enge und Tiefe ermöglicht es der $\operatorname{CRT}(X)$, unter Berücksichtigung der zeitlichen Dimension, eine Aussage zur Erneuerungskraft eines Wertpapiers zu treffen. Hierzu kann der $\operatorname{CRT}(X)$ für mehrere aufeinander folgende Zeitpunkte berechnet werden, um so die Entwicklung des $\operatorname{CRT}(X)$ über die Zeit zu analysieren. Gomber et al. (2015) untersuchen anhand einer Eventstudie, wie viel Zeit am deutschen Aktienmarkt nötig ist, bis die Liquidität nach einem großen Auftrag zu ihrem ursprünglichen Niveau zurückkehrt.

Bei der Berechnung des CRT $(X)$ ergeben sich zwei wesentliche Schwierigkeiten. Zum einen liegen die zur Berechnung nötigen Daten häufig nicht vollständig vor und zum anderen sind die Daten, sofern diese vorhanden sind, häufig nicht über einen langen historischen Zeitraum verfügbar. Aus diesem Grund wurden verschiedene Maße entwickelt, um die Liquidität aus den Transaktionspreisen zu schätzen. Wichtige Proxies, die in der empirischen Forschung Verwendung finden, sollen im nächsten Abschnitt kurz erläutert werden.

\subsubsection{Liquiditätsproxies}

Die in der Literatur verwendeten Liquiditätsproxies lassen sich in zwei Kategorien einteilen, die es zum Ziel haben, erstens die Geld-Brief-Spanne und zweitens den Preiseinfluss zu approximieren. Der Preiseinfluss gibt an wie stark sich die Ausführung eines Kauf-

${ }^{2}$ Eine präzise mathematische Darstellung zur Berechnung des CRT $(X)$ befindet in der Studie von Irvine et al. (2000) auf S. $7 \mathrm{f}$. 
oder Verkaufsauftrag auf den Preis eines Wertpapiers auswirkt. Da dieses Konzept nicht nur auf kleine Aufträge sondern auch auf große Aufträge anwendbar ist, kann mit dem Preiseinfluss die Dimension der Tiefe erfasst werden.

Eine grundlegende Arbeit zur Schätzung der Geld-Brief-Spanne wurde von Roll (1984) verfasst. In seiner Studie geht er von einem informationseffizienten Markt und stationären Preisveränderungen aus. Unter diesen Annahmen sowie der Unabhängigkeit der Preisveränderungen sollte der Mittelpunkt der Geld-Brief-Spanne, welcher auch als Fundamentalwert angesehen werden kann, zufällig schwanken. Die beobachtbaren Marktpreise sind jedoch nicht unabhängig voneinander, da jeder Handel entweder zum Geldoder Briefkurs abgeschlossen wird. Aus dieser Überlegung leitet Roll (1984) ein Maß für die Geld-Brief-Spanne ab. Bezeichnen $\Delta p_{t-1}$ und $\Delta p_{t}$ aufeinander folgende Preisveränderungen, kann die Geld-Brief-Spanne durch $\sqrt{-\operatorname{cov}\left(\Delta p_{t-1}, \Delta p_{t}\right)}$ approximiert werden.

In der Studie von Goyenko et al. (2009) finden sich viele unterschiedliche Varianten die Geld-Brief-Spanne zu approximieren. Neben dem klassischen Schätzer von Roll (1984) soll in dieser Arbeit ein weiterer aktueller Schätzer, der von Corwin \& Schultz (2012) entwickelte Hoch-Tief-Schätzer, vorgestellt werden. Dieser Schätzer benötigt wenig Rechenaufwand und eignet sich besser als der Roll-Schätzer zur Approximation der Liquidität eines Wertpapiers (Corwin \& Schultz (2012)). Die Studie von Schestag et al. (2013) zeigt für den US-Anleihemarkt, dass der Hoch-Tief-Schätzer einer der am besten geeigneten Schätzer zur Approximation der Geld-Brief-Spanne ist. Nach Corwin \& Schultz (2012) ist es unumstritten, dass Höchst- bzw. Tiefst-Kurse nahezu immer durch einen Kauf bzw. Verkauf zustande kommen. Damit beinhaltet das Verhältnis dieser beiden Kurse sowohl die Volatilität als auch die Geld-Brief-Spanne der Aktie. Corwin \& Schultz (2012) können einen Schätzer für die Geld-Brief-Spanne aus den Höchst- und Tiefstkursen ableiten, da lediglich die Varianz einer Aktie und nicht die Geld-Brief-Spanne proportional mit der Zeit ansteigt. Dieser Schätzer ist eine Funktion des Verhältnisses von Höchstund Tiefstkurs für zwei einzelne, aufeinander folgende Handelstage und des Verhältnisses von Höchst- und Tiefstkurs für eine zweitägige Handelsperiode.

Ein Maß zur Approximation des Preiseinflusses aus täglichen Kurs- und Volumendaten wird von Amihud (2002) entwickelt. Wird die Tagesrendite eines Wertpapiers mit $r_{t}$ und dessen tägliches Handelsvolumen in Dollar mit $H V_{t}$ bezeichnet, ist das Amihud-Maß 
(Amihud) des Wertpapiers für einen Beobachtungszeitraum von $n$ Tagen gegeben durch

$$
\text { Amihud }=\frac{1}{n} \sum_{t=1}^{n} \frac{\left|r_{t}\right|}{H V_{t}}
$$

und kann als tägliche Preisveränderung in Reaktion auf ein Handelsvolumen von einem Dollar interpretiert werden. Nach Goyenko et al. (2009) ist das Amihud-Maß am besten geeignet, um den Preiseinfluss zu erfassen. Die Studie von Goyenko et al. (2009) untersucht, ob die in der Forschung verwendeten Proxies tatsächlich in der Lage sind, Liquidität zu messen. Dazu werden Benchmarks sowohl für die Geld-Brief-Spanne als auch für den Preiseinfluss aus hochfrequenten Orderbuchdaten für den US-Markt berechnet. Goyenko et al. (2009) bestätigen, dass die aus täglichen Kurs- und Volumendaten berechneten Proxies sich zur Liquiditätsmessung eignen, wobei es schwieriger ist, den Preiseinfluss als die Geld-Brief-Spanne zu erfassen. Eine vergleichbare Studie von Fong et al. (2014) untersucht, anhand welcher Proxies die Liquidität auf internationalen Finanzmärkten am besten gemessen werden kann.

\subsection{Determinanten von Liquidität}

In diesem Abschnitt werden in der wissenschaftlichen Literatur diskutierte mögliche Ursachen für die Illiquidität von Finanzmärkten erläutert. Im Wesentlichen lassen sich drei Ursachen identifizieren: Handelskosten, Inventarkosten und Kosten asymmetrischer Information. Mit Handelskosten werden die Kosten bezeichnet, die bei der Bearbeitung von Kauf- und Verkaufsaufträgen entstehen. Nach Huang \& Stoll (1997) wird allgemein anerkannt, dass die Bereitsteller von Liquidität für die Handelskosten kompensiert werden müssen. Der Fokus modelltheoretischer Untersuchungen liegt daher überwiegend darauf die Illiquidität durch Inventarkosten und Kosten asymmetrischer Information zu erklären. Die Erläuterung entsprechender Modelle erfolgt im ersten und zweiten Teil dieses Abschnitts. Im letzten Teil wird die empirische Evidenz bezüglich der drei unterschiedlichen Kostenarten dargestellt.

\subsubsection{Inventarproblematik}

Durch das Eintreffen von Kauf- und Verkaufsaufträgen zu unterschiedlichen Zeitpunkten kann es an Börsen zu temporären Ungleichgewichten kommen. Die Auswirkungen 
dieser Ungleichgewichte für einen Dealermarkt werden modelltheoretisch von Garman (1976) analysiert. Im Rahmen dieses Modells wird ein risikoneutraler Market Maker betrachtet, der als Monopolist agiert und seinen Periodengewinn maximiert, indem er die Geld- und Briefkurse setzt zu denen er bereit ist Wertpapiere zu kaufen bzw. verkaufen. Die Investoren können nur über den Market Maker miteinander handeln. Das aggregierte Angebot und die aggregierte Nachfrage sind exogen für den Market Maker und er reagiert lediglich auf die eintreffenden Kauf- und Verkaufsaufträge, welche als unabhängige stochastische Prozesse modelliert werden. Aufgrund dieser Unabhängigkeit treffen die Kauf- und Verkaufsaufträge nicht synchron ein. Sofern Ungleichgewichte entstehen, ist der Market Maker verpflichtet, selbst entsprechende Positionen einzugehen, um das stetige Handeln zu ermöglichen.

Der Market Maker setzt die Geld- und Briefkurse lediglich einmal zu Beginn der Periode und besitzt ein bestimmtes Inventar. Ein negatives Inventar bedeutet die Insolvenz für den Market Maker. Die Gefahr für den Market Maker besteht darin, dass er in diesem Fall nicht weiter handeln und damit keinen Gewinn mehr erwirtschaften kann. Der Fokus des Modells liegt nicht auf dem Inventar des Market Makers, sondern wie die Illiquidität, in Form der Geld-Brief-Spanne, zustande kommt. Ein zentrales Ergebnis des Modells ist, dass diese Art von Marktstruktur eine Differenz zwischen Geld- und Briefkurs impliziert. Der Market Market muss den Briefkurs über den Geldkurs setzen, um die sichere Insolvenz zu vermeiden. Jedoch ist es dem Market Maker nicht möglich, unabhängig davon, welche Preise er setzt, die Insolvenz mit Sicherheit zu vermeiden.

Amihud \& Mendelson (1980) entwickeln ein Modell in dem wie in dem Modell von Garman (1976) ein risikoneutraler monopolistischer Market Maker seinen Gewinn durch das setzen der Geld-Brief-Spanne maximiert. Das Inventar beschreibt wieder eine Nebenbedingung, die der Market Maker einhalten muss und die seine Strategie Renditen zu generieren beeinflusst. Im Unterschied zu Garman (1976) setzt der Market Maker die Geld-Brief-Spanne nicht nur zu Beginn, sondern kann diese über die Zeit anpassen. Durch die Anpassung der Geld-und Briefkurse kann der Market Maker die Wahrscheinlichkeit beeinflussen, dass eine Nachfrage nach einem Wertpapier eingeht. Ist das Inventar des Market Makers gering, verkauft er nicht mehr und die Nachfrage wird nicht bedient. In diesem Fall erwirtschaftet der Market Maker keinen Gewinn. Daher gibt es eine optimale Inventarposition bei der die Monopolrendite des Market Makers am höchsten ausfällt. Ein weiteres Ergebnis des Modells ist, dass die Geld-Brief-Spanne wie bei Garman (1976) immer positiv ist. 
Einen anderen, risikobasierten Inventaransatz zur Erklärung der Geld-Brief-Spanne entwickelt Stoll (1978). In seinem Modell kann der Market Maker als risikoaverser Investor angesehen werden, welcher eine präferierte Portfolioposition hat und den Erwartungsnutzen seines Endvermögens maximiert. Um den unmittelbaren Handel zwischen Investoren zu ermöglichen, muss er Portfoliopositionen eingehen, die von seiner bevorzugten Position abweichen. Stoll (1978) nimmt an, dass der Market Maker diese Portfolioposition nicht absichern kann und damit Risiken ausgesetzt ist, die vergütet werden müssen. Im Fokus seiner Analyse stehen die Haltekosten des Market Makers, die aus den Risiken der nicht präferierten Portfolioposition resultieren.

Unter der Annahme, dass der Market Maker den wahren Wert des Wertpapiers kennt, leitet Stoll (1978) eine Funktion für die Haltekosten des Market Makers ab. Sowohl eine größere Risikoaversion des Market Makers als auch eine höhere Varianz des Wertpapierpreises führen zu größeren Haltekosten. Bei einer bereits bestehenden großen Inventarposition führt die Aufnahme weiteren Inventars ebenfalls zu höheren Kosten. Ein eingehender Auftrag erhöht oder verringert die Kosten, je nachdem ob der Market Maker sich durch den neuen Auftrag weiter von seiner präferierten Position entfernt oder sich ihr annähert. Steht der Market Maker im Wettbewerb wird er durch die gestellte Geld-Brief-Spanne lediglich für die Haltekosten entschädigt. O'Hara (2008) weißt darauf hin, dass die Geld-Brief-Spanne für einen risikoneutralen Market Maker entsprechend auf null sinken würde.

Ein weiteres risikobasiertes Modell zur Erklärung der Geld-Brief-Spanne entwickeln Ho \& Stoll (1981). In dem Modell setzt ein monopolistischer Market Maker mit konstanter relativer Risikoaversion die Geld-Brief-Spanne relativ zu einem exogen gegebenen wahren Preis und maximiert seinen Erwartungsnutzen. Anders als in der Studie von Stoll (1978) umfasst das Modell mehrere Perioden und die Portfoliorendite des Market Makers ist stochastisch. Der Fokus des Modells liegt auf den Unsicherheiten, denen der Market Maker ausgesetzt ist, und wie sich das damit verbundene Risiko auf seine Bereitschaft, Geld- und Briefkurse zu stellen, auswirkt.

Bei optimaler Preissetzung für den Market Maker kann die Geld-Brief-Spanne in eine risikoneutrale und eine für die Unsicherheit kompensierende Komponente unterteilt werden. Die risikoneutrale Komponente ist die Spanne, welche die erwarteten Gewinne maximiert. Sie ist umso kleiner, je elastischer die Nachfrage ist. Die zweite Komponente ist wie bei Stoll (1978) abhängig von der Risikoaversion des Market Makers und der Volatilität des Wertpapiers. 
Anders als Ho \& Stoll (1981) gehen O'Hara \& Oldfield (1986) in ihrem Modell weder von einem fixen, wahren Wertpapierpreis, noch von einem endlichen Beobachtungszeitraum aus. Sie analysieren die dynamische Preissetzung eines risikoaversen, nutzenmaximierenden, monopolistischen Market Makers, der über einen unbegrenzten Horizont agiert und seine Position zu keinem Zeitpunkt liquidiert. Der Market Maker ist zwei Formen von Unsicherheit ausgesetzt. Erstens besteht Unsicherheit über den Eingang von Aufträgen und zweitens ist der Wert seiner Inventarposition unsicher, da der Preis des Wertpapiers stochastisch ist.

Wie im Modell von Ho \& Stoll (1981) kann die resultierende Geld-Brief-Spanne unterteilt werden in eine risikoneutrale Komponente und eine Komponente, welche den Market Maker für die Risiken kompensiert. Ein wesentlicher Unterschied ist, dass im Modell von O'Hara \& Oldfield (1986) die Geld-Brief-Spanne nicht unabhängig vom Inventar des Market Makers ist. Zur Analyse der Auswirkungen des Inventars auf die Geld-Brief-Spanne unterstellen die Autoren, dass der Market Maker eine konstante absolute Risikoaversion besitzt. Wird nun das Optimierungsproblem des Market Makers gelöst zeigt sich, dass sowohl die Höhe als auch der Mittelpunkt der Geld-Brief-Spanne vom Wert des Inventars abhängig sind.

Die Studie von Ho \& Stoll (1983) trägt dem in der Realität existierenden Wettbewerb zwischen Market Makern Rechnung. Die Autoren entwickeln ein Modell, in dem die Market Maker mit anderen Market Makern und Investoren handeln. Die Investoren werden mit dem Market Maker handeln, welcher den besten Preis stellt. Folglich sollte die Preissetzung eines Market Makers die Aktionen der anderen Market Maker berücksichtigen. Ho \& Stoll (1983) lösen nicht dieses allgemeine Problem sondern lediglich eine vereinfachte Version des Modells, in der nur zwei Market Maker existieren und im Wettbewerb stehen. Darüber hinaus wird nur eine Periode und damit keine zeitliche Dimension des Problems betrachtet. Die resultierende Geld-Brief-Spanne hat eine einfache Struktur und hängt von der Varianz des Wertpapiers, den Handelskosten sowie der Risikoaversion des Market Makers ab. Der Wert des Inventars beeinflusst lediglich den Mittelpunkt der Geld-Brief-Spanne, nicht jedoch deren Höhe.

Ein besseres Verständnis der Determinanten der Geld-Brief-Spanne ist auch Gegenstand neuerer Studien. Jedoch hat nach Hasbrouck (2006) die Charakterisierung eines Market Makers über dessen Inventar an Relevanz verloren. Stattdessen schlägt er vor, die Inventarproblematik der Market Maker allgemeiner als Positionsmanagement zu inter- 
pretieren, da so die damit verbundenen Risiken auch für andere Investoren, welche z. B. in einem Ordermarkt als Liquiditätsanbieter agieren, von Bedeutung sind.

In dem Modell von Bollen et al. (2004) besitzt die Geld-Brief-Spanne neben der Inventarkomponente einen Anteil, welcher aus asymmetrischer Information resultiert. Um diesen Aspekt der Geld-Brief-Spanne besser zu verstehen, wird im nächsten Kapitel die Annahme aufgehoben, dass alle Marktteilnehmer die gleichen Informationen besitzen. Die Berücksichtigung der Informationsproblematik war ein bedeutender Schritt für die Forschung der Marktmikrostruktur (O'Hara (2008)).

\subsubsection{Asymmetrische Information}

Den Ausgangspunkt dafür, Informationsasymmetrien als Ursache für die Geld-BriefSpanne zu sehen, bildet eine Studie von Jack Treynor, welcher diese unter dem Pseudonym Bagehot (1971) veröffentlichte. In dieser Studie wird zwischen liquiditätsmotivierten Investoren ohne spezielle Informationen und informierten Investoren, welche private Informationen besitzen, unterschieden. Im Gegensatz zu den informierten Investoren, die nur am Markt agieren, sofern sie von ihren Informationen profitieren, ist der Market Maker zum Kaufen bzw. Verkaufen von Wertpapieren verpflichtet. Folglich ist der Market Maker bei einem Handel mit informierten Investoren schlechter gestellt und verliert im Durchschnitt. Daher setzt der Market Maker die Geld-Brief-Spanne so, dass er durch den Handel mit den uninformierten Investoren für diesen Verlust kompensiert wird.

Copeland \& Galai (1983) analysieren diese Idee als erste in einem formalen, modelltheoretischen Rahmen und zeigen, dass allein aus Informationsasymmetrien eine Geld-BriefSpanne resultiert. In ihrem statischen Modell mit nur einem Handelszeitpunkt kann jedoch nicht abgebildet werden, dass durch den Handelsprozess Informationen über den allgemein unbekannten Fundamentalwert öffentlich werden. Dieser Tatsache wird in einem Mehrperiodenmodell von Glosten \& Milgrom (1985) Rechnung getragen. Glosten \& Milgrom (1985) gehen in ihrem Modell von einem risikoneutralen Market Maker aus, der keinen Handelskosten- bzw. Inventarkosten ausgesetzt ist und aufgrund von Wettbewerb keinen Profit erwirtschaftet. Im Unterschied zu Copeland \& Galai (1983) werden die privaten Informationen in dem Mehrperiodenmodell von Glosten \& Milgrom (1985) nach einer Periode nicht offengelegt. Der Market Maker kann damit nicht unterscheiden, ob er mit einem informierten oder uninformierten Investor handelt. Die im Gleichgewicht gestellten Geld- und Briefkurse sind „regret-free“, d. h. der Market Maker glaubt, dass 
der Preis fair ist, unabhängig davon, ob es zu einem Kauf oder Verkauf kommt. Glosten \& Milgrom (1985) zeigen, dass die Erwartungen des Market Makers und der informierten Investoren konvergieren und damit alle Informationen im Preis widergespiegelt werden. Weitere Erkenntnisse aus dem Modell sind, dass die Geld-Brief-Spanne mit der Anzahl an informierten Investoren im Markt und der Qualität ihrer Informationen ansteigt. Darüber hinaus kann es zu Marktversagen kommen, wenn die informierten Investoren zu zahlreich oder ihre Informationen besonders gut sind.

Easley \& O’Hara (1987) entwickeln ein Modell ähnlich dem von Glosten \& Milgrom (1985), erweitern es aber dahingehend, dass Aufträge verschiedene Größen haben können. Easley \& O'Hara (1987) zeigen so, dass informierte Investoren es bevorzugen, große Mengen zu handeln, weil dadurch der Gewinn aus ihrer privaten Information am größten ist. Da dieses Phänomen nur bei informierten Investoren auftritt, nimmt der Market Maker große Handelsmengen als negative Information war und lässt große Aufträge zu schlechteren Preisen ausführen. Easley \& O'Hara (1987) finden damit einen informationsbasierten Erklärungsansatz dafür, dass große Mengen zu schlechteren Preisen gehandelt werden.

In den bisher beschriebenen informationsbasierten Modellen findet der Handel nacheinander statt, wobei lediglich ein Investor pro Zeitpunkt handeln kann. Eine weitere Klasse von informationsbasierten Modellen geht von strategisch handelnden Investoren aus, die den Zeitpunkt oder die Größe ihrer Aufträge wählen. Die grundlegende Arbeit für diese Modellklasse wurde von Kyle (1985) verfasst. Anders als bei Easley \& O'Hara (1987) berücksichtigt der strategisch handelnde Investor im Modell von Kyle (1985) den negativen Einfluss, den ein großer Auftrag auf den Preis hat.

Kyle (1985) entwickelt zunächst ein einperiodiges Modell in dem zufällig handelnde uninformierte Investoren und ein informierter Investor ihre Aufträge an einen risikoneutralen Market Maker übermitteln. Der Market Maker sieht nur die aggregierte Nachfrage und setzt basierend auf dieser Information den Preis fest, zu dem ein Handel abgeschlossen wird. Sofern ein Ungleichgewicht zwischen Kauf- und Verkaufsaufträgen besteht, handelt der Market Maker selbst und es kommt zur Markträumung. Ziel des risikoneutralen informierten Investors ist es, seinen Gewinn zu maximieren, indem er bei einer positiven (negativen) Information eine möglichst große Menge kauft (verkauft). Da der Market Maker nicht zwischen den Aufträgen von informierten und uninformierten Investoren unterscheiden kann, bilden die Aufträge der uninformierten Investoren eine Art Camouflage für die des informierten Investors. Kyle (1985) zeigt, dass die Liquidität 
proportional zur Höhe der zufälligen Aufträge der uninformierten Investoren und invers proportional zur Menge der privaten Information ist. Gemessen wird die Liquidität, die durch die Markttiefe repräsentiert wird, durch das sogenannte Kyle-lambda. Dabei gibt $\frac{1}{\lambda}$ die Auftragsgröße an, die benötigt wird um den Preis um einen Dollar steigen bzw. fallen zu lassen. In einer Modellvariante erweitert Kyle (1985) das Einperiodenmodell um mehrere Perioden, so dass es möglich ist, die Aufträge über die Zeit zu verteilen. ${ }^{3}$ Die zentralen Aussagen sind auch im Mehrperiodenmodell gültig.

Zur Klasse der informationsbasierten Modelle mit strategisch handelnden Investoren existiert eine große Anzahl wissenschaftlicher Studien, die das Kyle-Modell in verschieden Richtungen erweitern. Die Erweiterungen lassen sich in zwei wesentliche Richtungen einteilen. Der erste Strang analysiert die strategischen Entscheidungen mehrerer informierter Investoren im Rahmen mehrperiodiger Modelle (vgl. z.B. Holden \& Subrahmanyam (1992), Foster \& Viswanathan (1996) und Back et al. (2000)). Die Studien des zweiten Strangs beschäftigen sich mit dem strategischen Verhalten uninformierter Investoren und der Frage, wie diese ihre Aufträge zu einem bestimmen Zeitpunkt platzieren, um so die Verluste aus dem Handel mit informierten Investoren zu reduzieren (vgl. z.B. Admati \& Pfleiderer (1988), Seppi (1990) und Spiegel \& Subrahmanyam (1992)).

In einem neueren informationsbasierten Modell analysieren Cespa \& Foucault (2014) die Auswirkungen von Liquiditätsschocks und deren Bedeutung für die Liquiditätsbeziehung von Finanzmärkten. Ihr zentrales Argument besteht darin, dass der Market Maker eines Finanzmarktes die Preise auf einem verwandten Finanzmarkt als Informationsquelle nutzt. Im Rahmen eines Universums, welches aus zwei Wertpapieren A und B besteht, zeigen die Autoren, dass sich die Liquidität der Wertpapiere in die gleiche Richtung bewegt. Kommt es durch einen Liquiditätsschock zu einem Absinken der Liquidität von A, hat dies zur Folge, dass der Preis dieses Wertpapiers weniger informativ wird. Folglich ist der Market Maker für Wertpapier B aufgrund der schlechteren Informationen einer höheren Unsicherheit ausgesetzt, was sich in einer größeren Geld-Brief-Spanne bzw. Illiquidität des Wertpapiers B niederschlagen wird. Ihre Studie leistet darüber hinaus einen Beitrag zum Verständnis der Finanzmarktstabilität. Der ursprüngliche Liquiditätsschock kann, dadurch dass die gestiegene Illiquidität von Wertpapier B wiederum die Unsicherheit des Market Makers für Wertpapier A erhöht, eine Kettenreaktion auslösen, so dass der ursprüngliche Liquiditätsschock für das Wertpapier A verstärkt wird.

\footnotetext{
${ }^{3}$ Das Teilen großer Aufträge in kleinere und deren Aufteilung über die Zeit, findet in der Praxis häufige
} Anwendung, um den Preiseinfluss der Aufträge gering zu halten (Hasbrouck (2006)). 


\subsubsection{Empirische Evidenz}

Die relative Bedeutung der in der wissenschaftlichen Literatur identifizierten Determinanten der Geld-Brief-Spanne wird in verschiedenen Studien empirisch analysiert. Es besteht weitgehende Einigkeit darüber, dass ein großer Anteil der Geld-Brief-Spanne auf die Handelskosten zurückzuführen ist (vgl. z. B. Stoll (1989), George et al. (1991), Huang \& Stoll (1997) und Menyah \& Paudyal (2000)). Die Ergebnisse bzgl. der relativen Bedeutung von Inventarkosten und Kosten asymmetrischer Information sind dagegen gemischt.

Stoll (1989) zerlegt die Geld-Brief-Spanne in Anteile für Handelskosten, Inventarkosten und Kosten asymmetrischer Information. Unter Verwendung von quotierten Preisen der NASDAQ schätzt Stoll (1989), dass lediglich $10 \%$ der Geld-Brief-Spanne auf die Inventarkosten zurückzuführen sind. Auch in der Studie von Huang \& Stoll (1997) wird die Geld-Brief-Spanne in drei Anteile für die unterschiedlichen Kostenarten zerlegt. Die Autoren können anhand von quotierten Preisen der NYSE ebenfalls lediglich eine geringe Bedeutung von Inventarkosten nachweisen. Vergleichbare Ergebnisse finden auch die Studien von Madhavan \& Smidt (1991) und Manaster \& Mann (1996), die Transaktionspreise der NYSE bzw. der Chicago Mercantile Exchange untersuchen. Im Gegensatz zu diesen Ergebnissen dokumentiert die Studie von Bollen et al. (2004) für quotierte Preise der NASDAQ, dass die Inventarkosten den größten Anteil der Geld-Brief-Spanne ausmachen.

Den Anteil der Kosten asymmetrischer Information schätzt Stoll (1989) auf 43\%. Eine große Bedeutung haben diese Kosten auch nach Menyah \& Paudyal (2000). Für quotierte Preise der London Stock Exchange schätzen die Autoren den Anteil der Kosten asymmetrischer Information auf $47 \%$. Eine signifikante jedoch geringe Bedeutung der Kosten asymmetrischer Information findet dagegen die Studie von Huang \& Stoll (1997). Auch die Studie von George et al. (1991) zeigt, dass nur ein kleiner Anteil der Geld-BriefSpanne auf diese Kostenart zurückzuführen ist. Die empirische Evidenz der Studie von Bollen et al. (2004) legt ebenfalls eine geringe Relevanz von asymmetrischer Information nahe. 


\subsection{Auswirkungen von Liquidität}

\subsubsection{Preiseffekte}

Theoretische und empirische Studien stützen die These, dass Liquidität ein bewerteter Faktor auf Finanzmärkten ist. In diesem Abschnitt wird zunächst kurz darauf eingegangen, welche unterschiedlichen Formen von Liquidität auf Finanzmärkten eingepreist werden. Im Anschluss daran werden die Auswirkungen von Liquidität auf Preise an Futures- und Optionsmärkten beschrieben.

\subsubsection{Allgemeine Effekte auf Preise}

Wie sich Liquidität im Gleichgewicht auf Preise und Renditen von Wertpapieren auswirkt ist seit langem Gegenstand der Finanzforschung. ${ }^{4}$ Bereits in den 1980er Jahren wird in der Studie von Amihud \& Mendelson (1986) dargelegt, dass Investoren aufgrund der höheren Handelskosten bei illiquiden Wertpapieren in Form einer höheren erwarteten Rendite kompensiert werden müssen. Empirisch weisen sie einen positiven Zusammenhang zwischen der durchschnittlichen Aktienilliquidität und den Aktienrenditen nach. In einer neueren Studie finden Pastor \& Stambaugh (2003) empirische Evidenz, dass die Marktliquidität eingepreist wird. ${ }^{5}$

Acharya \& Pedersen (2005) entwickeln ein liquiditätsadjustiertes Capital Asset Pricing Model (CAPM), welches in der Lage ist diese empirischen Befunde zu erklären. In ihrem Gleichgewichtsmodell stehen nutzenmaximierende Investoren im Wettbewerb und sind beim Handel von Wertpapieren Illiquiditätskosten ausgesetzt. Eine Innovation von Acharya \& Pedersen (2005) ist es, dass die Illiquiditätskosten über die Zeit schwanken. Diese Schwankungen werden als Liquiditätsrisiko interpretiert. Im Rahmen ihres Modells zeigen die Autoren, dass die erwartete Rendite von vier verschiedenen Risikoformen, die sich alle in Form von Kovarianzen ausdrücken lassen, abhängig ist. Neben dem

\footnotetext{
${ }^{4}$ Da eine vollständige Darstellung der bestehenden Literatur zu den Auswirkungen von Liquidität auf die Bewertung von Wertpapieren nicht Ziel dieser Arbeit ist, sei auf den Übersichtsartikel von Amihud et al. (2005) hingewiesen, der sowohl die theoretische Modellierung als auch die empirische Evidenz $\mathrm{zu}$ diesem Thema behandelt.

${ }^{5}$ Weitere Studien, die empirische Evidenz dafür finden, dass Liquidität ein bewerteter Faktor ist, sind u. a. Amihud (2002), Bekaert et al. (2007), Korajczyk \& Sadka (2008), Watanabe \& Watanabe (2008) und Hasbrouck (2009).
} 
Marktrisiko, gemessen durch die Kovarianz der Wertpapierrendite mit der Marktrendite, gibt es drei unterschiedliche Formen des Liquiditätsrisikos. Die erwartete Rendite wird positiv von der Kovarianz der Wertpapier- und Marktliquidität, negativ von der Wertpapierrendite und der Marktliquidität sowie negativ von der Wertpapierliquidität und der Marktrendite beeinflusst. Empirisch zeigt sich, dass insbesondere bei Aktien mit geringer durchschnittlicher Liquidität der Einfluss dieser drei Liquiditätsrisiken stark ausgeprägt ist, wobei die letzte Form des Liquiditätsrisikos den größten Anteil hat.

\subsubsection{Effekte auf Futurespreise}

In der bestehenden Literatur finden sich im Wesentlichen zwei Gründe, welche die Existenz von Risikoprämien am Markt für Aktienindexfutures erklären. Der erste Grund wird im systematischen Risiko gesehen. Auf friktionslosen Märkten ist der Cost-of-Carry-Preis eines Indexfutures perfekt an den Index der Basiswertpapiere gekoppelt und hat daher das gleiche systematische Risiko wie der Index der Basiswertpapiere. Gegenüber diesem Risiko können sich die Investoren nicht absichern und müssen daher in Form von Risikoprämien entschädigt werden.

Der zweite Grund liegt in Risiken, die aus Marktfriktionen resultieren. Bei Marktfriktionen kann die Nachfrage nach Futures als Hedginginstrument („hedging pressure“) Auswirkungen auf die Risikoprämien am Futuresmarkt haben, da sich Investoren aufgrund der Marktfriktionen nicht gegenüber den Risiken ihrer Futuresposition absichern können. ${ }^{6}$ De Roon et al. (2000) entwickeln ein Modell in dem die erwarteten Futuresrenditen nicht nur vom „hedging pressure“ am eigenen Markt, sondern darüber hinaus vom „hedging pressure“ an anderen Märkten abhängen. In ihrer empirischen Analyse untersuchen sie 20 Futuresmärkte und zeigen, dass Futuresrenditen signifikant vom eigenen und dem „hedging pressure“ der anderen Märkte beeinflusst werden.

Explizit mit den Auswirkungen von Illiquidität auf die Preise am Futuresmarkt beschäftigt sich die Studie von Roll et al. (2007). Die Autoren untersuchen, ob ein Zusammenhang zwischen der Liquidität des S\&P 500 Index und den Abweichungen von der No-Arbitrage Beziehung zwischen Indexfutures und dem Index der Basiswertpapiere besteht. Ihrer Argumentation nach sollten Abweichungen vom No-Arbitrage Preis in

\footnotetext{
${ }^{6}$ Verschiedene theoretische Studien modellieren den Einfluss von systematischem Risiko und „hedging pressure" auf die Risikoprämien am Futuresmarkt (vgl. z.B. Stoll (1979) und Hirshleifer (1989)). Bessembinder \& Seguin (1993) finden empirische Evidenz für die Bedeutung der beiden Faktoren.
} 
Verbindung mit der Liquidität stehen, da eine hohe Liquidität das Ausnutzen von Fehlbewertungen erleichtert. Andererseits sollten Fehlbewertungen Arbitrageure anziehen und dadurch wiederum die Liquidität beeinflussen. Empirisch zeigen sie, dass Illiquidität eine Bewegung der Fehlbewertung zum friktionslosen Ideal von null erschwert. Des Weiteren finden sie Evidenz für die gegenseitige Beeinflussung von Fehlbewertung und Liquidität in Form einer Grangerkausalität. Eine Grangerkausalität der beiden Faktoren wird ebenfalls von Kadapakkam \& Kumar (2013) für den indischen SSF-Markt nachgewiesen. Lien et al. (2013) erweitern die Studie von Roll et al. (2007), indem sie eine nichtlineare Beziehung zwischen der Basis, definiert als Differenz der Preise von Futures und Basiswertpapier, und der S\&P 500 Liquidität untersuchen. Ihre empirische Analyse kommt zu dem Ergebnis, dass ein Anstieg der Illiquidität ebenfalls mit einem Anstieg der Basis einhergeht. ${ }^{7}$ Sinkt die Illiquidität, kommt es jedoch nicht immer zu einer Reduktion der Basis.

\subsubsection{Effekte auf Optionspreise}

Unter der Annahme friktionsloser Märkte kann eine Option nach Black \& Scholes (1973) durch ein Portfolio aus Aktien und risikolosem Instrument perfekt repliziert werden. Bestehen am Kassamarkt Friktionen, kann dies Kosten bei der Replikation verursachen und damit Auswirkungen auf den Optionspreis haben. In diesem Abschnitt werden direkt die Auswirkungen von Friktionen auf den Optionspreis beschrieben.

Die Studie von Gârleanu et al. (2009) findet allgemeine Evidenz zu den Auswirkungen von Friktionen auf den Optionspreis. Sie untersuchen, wie sich Nachfrageüberschüsse nach Optionen bei Marktunvollständigkeiten auf deren Preise auswirken. Dazu modellieren sie einen risikoaversen, im Wettbewerb stehenden Market Maker, der das Angebot an Optionen sicherstellt. Der Market Maker kann seine Position aufgrund von diskretem Handeln, stochastischer Volatilität und Preissprüngen im Basiswertpapier nicht perfekt absichern. Die Autoren zeigen, dass ein Anstieg der Nachfrageüberschüsse zu einem Anstieg des Optionspreises führt. Dieser Anstieg ist proportional zur Varianz der Risiken, die vom Market Maker nicht gehedgt werden können. Anhand eines Datensatzes von S\&P 500 Indexoptionen und Optionen auf Einzelaktien finden sie empirische Evidenz

\footnotetext{
${ }^{7}$ Es ist zu beachten, dass ein Anstieg der Basis nicht notwendigerweise mit einem Anstieg der Fehlbewertung einhergeht. So könnte ein Anstieg der Basis auch durch Zinsänderungen oder Dividendenzahlungen erklärt werden.
} 
dafür, dass Nachfrageüberschüsse das Level der Optionspreise erklären können. Die Studie von Bollen \& Whaley (2004) findet ebenfalls für einen Datensatz von S\&P 500 Indexoptionen und Optionen auf Einzelaktien empirische Evidenz, dass Veränderungen in der Nachfrage nach Optionen Auswirkungen auf die implizite Volatilität und damit auf die Optionspreise haben.

Neben diesen Studien zur allgemeinen Evidenz existieren verschiedene Studien die spezielle Evidenz für die Auswirkungen von Illiquidität auf den Optionspreis finden. Die frühe Literatur modelliert die Illiquidität am Kassamarkt in Form von Transaktionskosten und analysiert deren Auswirkungen auf die Replikationskosten und den Optionspreis. Auf einem illiquiden Kassamarkt würden die Transaktionskosten bei stetiger Adjustierung der Gewichte im Replikationsportfolio ins Unendliche steigen. Leland (1985) entwickelt eine alternative Strategie, die von den Transaktionskosten und der Häufigkeit der Umschichtungen im Replikationsportfolio abhängt. Die Strategie ermöglicht es die Transaktionskosten zu beschränken und wird umso präziser je häufiger umgeschichtet wird. Mit dem Einfluss von Transaktionskosten auf die Kosten der Replikation beschäftigt sich auch die Studie von Boyle \& Vorst (1992). In ihrem diskreten Mehrperiodenmodell liegen diese Kosten und damit die Optionspreise über denen von Leland (1985).

In den neueren Studien von Liu \& Yong (2005) und Cetin et al. (2006) wird die Illiquidität des Basiswertpapiers explizit modelliert und ihr Einfluss auf Optionspreise untersucht. In beiden Modellen entsteht bei der Replikation einer europäischen CallOption aufgrund der Illiquidität am Kassamarkt ein Preiseinfluss, der die Kosten für die Replikation erhöht. ${ }^{8}$ Im Modellrahmen von Liu \& Yong (2005) ist es möglich die Option exakt zu replizieren. Sie zeigen, dass auch bei kleinem Preiseinfluss die Replikationskosten signifikant über dem Black-Scholes-Preis liegen. Die von Cetin et al. (2006) entwickelten diskreten Handelsstrategien sind dagegen nicht in der Lage die Option perfekt zu replizieren, da sie berücksichtigen, dass in der Realität nicht stetig gehandelt werden kann. Empirisch zeigen die Autoren, dass selbst bei optimaler Hedgingstrategie die Liquiditätskosten einen signifikanten Teil des Optionspreises ausmachen.

Weitere empirische Studien zu den Auswirkungen der Illiquidität auf Optionspreise berücksichtigen sowohl die Illiquidität am Aktien- als auch am Optionsmarkt. Die in der Studie von Chou et al. (2011) dokumentierten hohen Preise von liquiden Optionen sind konsistent mit der Illiquiditätsprämien-Hypothese von Amihud \& Mendelson (1986). Die

\footnotetext{
${ }^{8}$ Weitere Studien, die die Auswirkungen eines Preiseinflusses auf die Replikationen von Optionen untersuchen, sind u. a. Frey (1998) und Frey (2000).
} 
Autoren finden darüber hinaus Evidenz, dass die Preise von Optionen auf illiquide Aktien höher sind als die Preise von Optionen auf liquide Aktien. Auch die Studien von Christoffersen et al. (2014) und Karakaya (2014) finden Evidenz für einen positiven Einfluss der Aktienilliquidität auf Optionspreise. Zu unterschiedlichen Ergebnissen kommen die beiden Studien dagegen in Bezug auf die Auswirkungen der Optionsliquidität. Die Ergebnisse von Christoffersen et al. (2014) implizieren eine Illiquiditätsprämie für Käufer von Optionen. Nach der Studie von Karakaya (2014) sollten dagegen die Verkäufer von Optionen eine Illiquiditätsprämie verdienen.

\subsubsection{Liquiditätseffekte}

Ziel dieses Abschnitts ist es, die Liquiditätszusammenhänge von Kassa- und Derivatemärkten zu erläutern. Insbesondere wird die Übertragung von Liquidität beschrieben, wobei der Fokus auf der Beziehung der Liquidität zweier Märkte zueinander liegt und weniger darauf, welcher Markt Ausgangspunkt der Liquiditätsübertragung ist. Das Kapitel gliedert sich in Abschnitte zur Liquidität auf Futures-, Options- und Exchange-TradedFunds- (ETF) Märkten.

\subsubsection{Futuresmärkte}

Subrahmanyam (1991) und Jegadeesh \& Subrahmanyam (1993) untersuchen Migrationseffekte von Investoren am Beispiel der Einführung von S\&P 500 Indexfutures und finden Evidenz für eine inverse Liquiditätsbeziehung des Futures- und zugrundeliegenden Aktienmarktes.

Die Einführung eines neuen Marktes für Indexfutures stellt eine besondere Art von Liquiditätsschock dar: Zunächst ist der Futuresmarkt nicht existent und damit perfekt illiquide. Mit dem Handelsbeginn von Indexfutures entsteht am Futuresmarktes Liquidität. Folglich bildet dieses Ereignis einen geeigneten Rahmen zur Analyse der Frage, wie ein Anstieg der Liquidität am Futuresmarkt auf die Liquidität am Kassamarkt wirkt.

Subrahmanyam (1991) analysiert die Migrationseffekte von Investoren nach Einführung eines Marktes für Aktienindexfutures modelltheoretisch. Dazu erweitert er das Modell von Kyle (1985) um mehrere Aktien und berücksichtigt informierte Investoren, welche auf Basis ihrer privaten Information handeln, und uninformierte Investoren deren 
Handelsmotive außerhalb der Finanzmärkte bestimmt werden. ${ }^{9}$ Ziel der uninformierten Investoren ist es, ein Aktienportfolio zu handeln und dabei die Kosten, welche aus dem Handel mit informierten Investoren entstehen, zu minimieren. Der Handel eines Aktienportfolios kann entweder über die Einzelaktien oder einen Aktienindexfutures erfolgen. Unter der Annahme, dass informierte Händler lediglich aktienspezifische Informationen besitzen, zeigt Subrahmanyam (1991), dass die Kosten aus asymmetrischer Information für die uninformierten Investoren im Markt des Aktienindexfutures geringer sind. Auch wenn die informierten Investoren über Informationen zu systematischen Faktoren verfügen, sind die Verluste der uninformierten Investoren am neuen Markt geringer. Dies hat zur Folge, dass uninformierte Investoren ihr Handeln auf den Markt des Aktienindexfutures verlagern.

Empirisch werden die Auswirkungen der Einführung von S\&P Indexfutures auf die Liquidität am Kassamarkt von Jegadeesh \& Subrahmanyam (1993) analysiert. Die Autoren entwerfen zwei unterschiedliche Szenarien, wie sich die Einführung von Indexfutures auf die Liquidität am Kassamarkt auswirken kann. Einerseits könnten die Informationsasymmetrien am Kassamarkt durch die Migration von uninformierten Investoren verstärkt werden. Für diese gestiegenen Informationsasymmetrien müssten die Market Maker am Kassamarkt entschädigt werden, was durch das Setzen einer größeren GeldBrief-Spanne erreicht werden könnte. Andererseits könnte der neue Futuresmarkt es den Market Makern ermöglichen, sich gegenüber ihren Inventarrisiken besser abzusichern. Nach diesem Hedgingargument sollte die Einführung der neuen Indexfutures eine Reduktion der Geld-Brief-Spanne zur Folge haben. Jegadeesh \& Subrahmanyam (1993) zeigen, dass sich die Illiquidität auf dem Kassamarkt, gemessen durch die Geld-BriefSpanne, mit der Eröffnung des Futuresmarktes erhöht.

In der bestehenden Literatur wurde nur das Ereignis der Einführung von Indexfutures analysiert, die Liquiditätsbeziehungen zwischen einem bereits etablierten Futures- und Kassamarkt jedoch nicht. Für einen bereits länger existierenden Futuresmarkt verliert das Argument von Subrahmanyam (1991) für eine inverse Liquiditätsbeziehung möglicherweise an Bedeutung, da die uninformierten Investoren bereits auf dem Futuresmarkt handeln und es nicht mehr zu bedeutenden Wechseln zwischen den beiden Märkten kommt. Ein Markt, dessen Liquiditätsbeziehungen bisher noch nicht untersucht wurden, ist der Markt für SSF. Auch für diesen Markt scheint das informationsbasierte Argument weniger relevant zu sein, da ein SSF ein Kontrakt auf eine spezielle Aktie ist und

\footnotetext{
${ }^{9}$ Handelsmotive können z. B. in Steuerplanungen oder dem Bedarf nach direktem Konsum liegen.
} 
damit auf beiden Märkten ähnliche Informationsasymmetrien vorliegen sollten. Wichtiger dagegen scheint das Hedgingargument von Jegadeesh \& Subrahmanyam (1993), da insbesondere SSF für eine Absicherung gegenüber Risiken im Basiswertpapier geeignet sind.

\subsubsection{Optionsmärkte}

Insbesondere Studien zur Liquidität von Optionsmärkten finden Hinweise für eine gleichgerichtete Bewegung der Liquidität auf Kassa- und Derivatemärkten. Der Großteil der Studien sieht den Ausgangspunkt der Liquiditätsübertragung am Kassamarkt. Zunächst werden die Studien erläutert, die die Auswirkungen von steigender oder fallender Liquidität am Kassamarkt auf die Liquidität am Optionsmarkt untersuchen. Ein zentraler Punkt dieser Studien sind die Hedgingkosten denen die Market Maker am Optionsmarkt ausgesetzt sind. Im Anschluss daran werden die Folgen von Liquiditätsschocks durch Leerverkaufsverbote am Kassamarkt beschrieben. Abschließend erfolgt eine kurze Darstellung der Studien, die einen Einfluss der Optionsliquidität auf die Kassamarktliquidität dokumentieren.

In einer grundlegenden Studie erläutern Cho \& Engle (1999) weshalb eine Geld-BriefSpanne am Derivatemarkt auch dann existiert, wenn der Market Maker keinen Inventarrisiken oder Kosten aus asymmetrischer Information ausgesetzt ist. Sie argumentieren, dass der Market Maker seine Position über den Kassamarkt absichert und ihm dabei Kosten aufgrund der Illiquidität des Kassamarktes entstehen, welche er in Form einer Geld-Brief-Spanne an den Derivatemarkt weitergibt. Steigt die Illiquidität am Kassamarkt, erhöhen sich die Hedgingkosten des Market Makers, was dazu führt, dass er die Geld-Brief-Spanne am Derivatemarkt erhöht. Folglich bewegt sich die Illiquidität nach der Theorie von Cho \& Engle (1999), welche sie „derivative-hedge-theorie“ nennen, in die gleiche Richtung. Anhand eines Datensatzes von S\&P 100 Indexoptionen vom Mai 1993 finden sie empirische Evidenz für ihre Theorie. Sie zeigen, dass die Geld-Brief-Spanne am Optionsmarkt in einem signifikanten, positiven Zusammenhang zu der Geld-BriefSpanne am Aktienmarkt und den Kosten, welche beim Aufsetzen eines Hedgeportfolios anfallen, steht. Landsiedl (2005) untersucht einen Datensatz der Wiener Börse AG vom Juni 2003 bis zum Dezember 2003 und findet ebenfalls empirische Evidenz für die „derivative-hedge-theorie“.

Kaul et al. (2004) erweitern die Studie von Cho \& Engle (1999), indem sie nicht nur die 
Kosten für das Aufsetzen eines Hedgeportfolios berücksichtigen (Hedgingkosten), sondern darüber hinaus die Kosten, welche sich durch das Adjustieren der Portfoliogewichte über die Zeit ergeben (Rebalancingkosten). Sie analysieren Aktienoptionen, die im Februar 1995 an der Chicago Board of Option Exchange (CBOE) gehandelt wurden, und zeigen, dass die Variablen für Hedging- und Rebalancingkosten in einem positiven Zusammenhang zu der Geld-Brief-Spanne am Optionsmarkt stehen. Eine weitere Ursache für die Geld-Brief-Spanne am Optionsmarkt sehen Kaul et al. (2004) in der asymmetrischen Information. Sie argumentieren, dass, sofern informierte Investoren simultan im Options- und dem zugrundeliegenden Aktienmarkt handeln, deren Verhalten sowohl die Geld-Brief-Spanne am Aktien- als auch am Optionsmarkt beeinflusst. Folglich sind Market Maker am Optionsmarkt, die sich nicht gegen die Kosten der asymmetrischen Information absichern können, den gleichen Informationsnachteilen wie die Market Maker am Kassamarkt ausgesetzt. Für ihren Datensatz können Kaul et al. (2004) einen positiven Zusammenhang zwischen den Kosten der asymmetrischen Information am Kassamarkt und der Geld-Brief-Spanne am Optionsmarkt nachweisen. Die Ergebnisse anderer empirischer Studien zu dieser Fragestellung sind jedoch gemischt. Nach Vijh (1990) und Cho \& Engle (1999) sind Markt Maker im Optionsmarkt keinen signifikanten Kosten aus asymmetrischer Information ausgesetzt. Die Studien von Easley et al. (1998) und Cao et al. (2005) legen dagegen die Existenz informierter Investoren am Optionsmarkt nahe.

Anhand eines größeren US-Optionsdatensatzes und einer anderen Methodik zur Messung der Rebalancingkosten als Kaul et al. (2004) weisen Engle \& Neri (2010) ebenfalls einen positiven Einfluss der Hedging- und Rebalancingkosten auf die Geld-Brief-Spanne am Optionsmarkt nach. Ein positiver Zusammenhang der beiden Variablen und der Geld-Brief-Spanne am Covered-Warrant-Markt in Italien wird von Petrella (2006) dokumentiert. In einer neueren Studie für den US-Markt bestätigen Goyenko et al. (2014) den positiven Einfluss von Hedging- und Rebalancingkosten auf die Geld-Brief-Spanne von Aktienoptionen. In ihrer Studie dokumentieren die Autoren eine größere Bedeutung der Rebalancing- gegenüber den Hedgingkosten. Darüber hinaus zeigen sie, dass Proxies für Inventarrisiken und Kosten adverser Selektion ebenfalls einen positiven Einfluss auf die Illiquidität am Optionsmarkt haben.Weitere empirische Evidenz für die komplementäre Sichtweise der Liquidität am Aktien- und Optionsmarkt findet die Studie von Christoffersen et al. (2014).

Eine spezielle Form des Liquiditätsschocks am Aktienmarkt stellt das Verbot von Leer- 
verkäufen dar. Wie die Liquidität am Optionsmarkt auf ein Leerverkaufsverbot am Aktienmarkt reagiert ist nicht offensichtlich, da unklar ist, ob Aktien und Optionen in diesem Fall als Komplemente oder Substitute zu sehen sind. Figlewski \& Webb (1993) betonen den Substitutionseffekt, indem sie argumentieren, dass das Kaufen von Put-Optionen durch Investoren, die Leerverkaufsbeschränkungen ausgesetzt sind, als eine alternative Handelsstrategie genutzt werden kann, um die gewünschte Position einzugehen. In ihrer Studie finden sie Evidenz dafür, dass Optionsmärkte die Effekte von Leerverkaufsbeschränkungen verringern. ${ }^{10}$

Grundy et al. (2012) weisen dagegen auf einen komplementären Aspekt der beiden Finanzinstrumente hin. Die Autoren formulieren die Hypothese, dass ein Market Maker durch den Verkauf von Put-Optionen höheren Risiken ausgesetzt ist, da seine Fähigkeit, sich über den Kassamarkt abzusichern, durch das Leerverkaufsverbot eingeschränkt wird. In ihrer empirischen Studie untersuchen Grundy et al. (2012) die Auswirkungen des Leerverkaufsverbots auf Finanzaktien durch US-Regulatoren während des Zeitraums vom 19. September bis zum 8. Oktober 2008. Die Autoren dokumentieren einen Anstieg der Geld-Brief-Spanne und einen Rückgang des Handelsvolumens am Optionsmarkt während der Periode der Leerverkaufsbeschränkungen. Der Anstieg der Geld-Brief-Spanne kann dabei nicht durch andere mögliche Treiber wie die gestiegene Volatilität oder den Preisverfall am Aktienmarkt erklärt werden. Sie folgern aus diesem Resultat, dass der komplementäre Aspekt von Aktien und Optionen überwiegt. Darüber hinaus zeigen sie, dass Investoren nicht auf Indexfutures-, SSF- oder ETF-Märkte ausweichen, um Shortpositionen gegenüber leerverkaufsbeschränkten Aktien einzugehen. $\mathrm{Zu}$ vergleichbaren Ergebnissen kommt die von Grundy et al. (2012) unabhängige Studie von Battalio \& Schultz (2011). Neben einem starken Anstieg der Geld-Brief-Spannen von Optionen auf leerverkaufsbeschränkte Aktien zeigen sie, dass die Qualität der Auftragsausführung abnimmt. Mit der Einführung des Leerverkaufsverbots werden Kaufaufträge (Verkaufsaufträge) zu Kursen ausgeführt, die signifikant über (unter) den gestellten Brief- und Geldkursen liegen.

Bisher wurde der Ausgangspunkt der Liquiditätsübertragung am Kassamarkt gesehen. Die Liquiditätsübertragung kann jedoch auch vom Optionsmarkt ausgehen. Insbesondere Studien zur Einführung neuer Optionen untersuchen diesen Kausalzusammenhang. Die Studie von Damodaran \& Lim (1991) findet Evidenz für eine gesunkene Geld-Brief-

10 Auch die Studien von Danielsen \& Sorescu (2001) und Blau \& Wade (2013) finden Evidenz für diesen Zusammenhang. 
Spanne der Aktien nach der Einführung von Einzeloptionen. Kumar et al. (1998) finden ebenfalls empirische Evidenz für einen Rückgang der Geld-Brief-Spanne der Aktien. Die Autoren zeigen darüber hinaus, dass die Komponente der Geld-Brief-Spanne, die auf adverse Selektion zurückzuführen ist, abnimmt und die Markttiefe ansteigt. Fedenia \& Grammatikos (1992) zeigen, dass die Einführung von Einzeloptionen insbesondere bei den weniger liquiden Aktien zu einer Reduktion in der Geld-Brief-Spanne führt. Kumar et al. (1995) untersuchen die Einführung von Indexoptionen und dokumentieren auch für diese Art von Optionen eine Reduktion der Geld-Brief-Spanne der zugrundeliegenden Aktien. Die empirische Evidenz zur Einführung von Optionen unterstützt damit ebenfalls die komplementäre Sichtweise von Aktien- und Optionsmärkten.

\subsubsection{ETF-Märkte}

Eine positive Beziehung zwischen der Liquidität eines ETF und seines Benchmarkindex wird in der Praxis häufig als gegeben angenommen (Calamia et al. (2014)). Diese Sichtweise ist u. a. darauf zurückzuführen, dass ein Index traditionell eins zu eins nachgebildet wird. Durch diese Art der Nachbildung entsteht ein Preiseinfluss, der dem Preiseinfluss entspricht, wenn das Aktienportfolio am Kassamarkt gekauft werden würde. Modelltheoretisch wird die Liquiditätsbeziehung von ETFs und ihren Benchmarkindizes von Calamia et al. (2014) analysiert. Im Rahmen ihres inventarbasierten Modells wird ein risikoaverser Market Maker für den ETF-Markt betrachtet, der seine Position am Ende des Beobachtungszeitraums glattstellen muss. Das Glattstellen der ETF-Position ist mit einem Handel am Aktienmarkt verbunden, wodurch Kosten aufgrund von Illiquidität am Kassamarkt verursacht werden.

Eine erste, aus dem Modell abgeleitete Hypothese besagt, dass ETFs auf illiquide Aktienindizes ebenfalls eine höhere Illiquidität aufweisen sollten. Darüber hinaus sollte nach dem Modell die Illiquidität des ETFs mit der Volatilität des Benchmarkindex und der Risikoaversion des Market Makers ansteigen. Empirisch weisen Calamia et al. (2014) einen positiven Zusammenhang zwischen der ETF Geld-Brief-Spanne und der Indexvolatilität nach. Die Geld-Brief-Spanne des Benchmarkindex ist jedoch nur für die GeldBrief-Spanne von ETFs mit geringen Handelsvolumina eine bedeutende Determinante. 


\section{Liquiditäts- und Preiseffekte am Forwardmarkt}

Ziel dieses Kapitels ist es modelltheoretisch zu analysieren, wie sich die Illiquidität eines Kassamarktes auf den Markt für Forwards überträgt. Im Rahmen eines Gleichgewichtsmodells werden verschiedene Faktoren identifiziert, die die Liquiditätsübertragung beeinflussen. Zunächst wird eine geschlossene Bewertungsgleichung für den Forwardpreis in Abhängigkeit der Forwardnachfrage hergeleitet. Diese Bewertungsgleichung bildet die Grundlage für ein Illiquiditätsmaß am Forwardmarkt, welches die Analyse der Transmissionseffekte vom Kassa- auf den Forwardmarkt ermöglicht.

Im Fokus des Modells steht ein repräsentativer, risikoaverser Market Maker für den Forwardmarkt. Der Handel am Forwardmarkt muss dabei nicht über den Market Maker erfolgen. Die Investoren können untereinander handeln und die Aufgabe des Market Makers besteht darin, den Nachfrageüberhang auszugleichen. Folglich kann mit dem Modell sowohl ein Dealer- als auch ein Hybridmarkt beschrieben werden. Durch seine Forwardposition ist der Market Maker Inventarrisiken ausgesetzt und kann sich gegenüber diesen absichern, was mit Hedgingkosten verbunden ist. Im Rahmen des Modells wägt der Market Maker zwischen Inventarrisiken und Hedgingkosten ab. Auswirkungen von asymmetrischen Informationen sind dagegen nicht Gegenstand des Modells.

Im ersten Teil des Kapitels wird die Illiquidität am Terminmarkt in einem Einperiodenmodell untersucht und es werden unterschiedliche Aspekte anhand verschiedener Modellvarianten analysiert. Das Modell wird im zweiten Teil des Kapitels um eine Periode erweitert. Im Rahmen des Mehrperiodenmodells werden die Hedgingstrategie des Market Makers sowie die Preis- und Liquiditäteffekte von Kontrakten unterschiedlicher Laufzeit analysiert.

\subsection{Einperiodenmodell}

Bereits anhand des Einperiodenmodells lassen sich wesentliche Treiber der Illiquidität am Forwardmarkt identifizieren und empirisch zu überprüfende Hypothesen zur Liquiditätsübertragung ableiten. Der zunächst dargestellte Basisfall für das Einperiodenmodell 
dient dazu, die dem Modell zugrunde liegende Struktur zu erklären. In verschiedenen Erweiterungen des Basisfalls wird der Kassamarkt auf unterschiedliche Weise modelliert und die damit verbundenen Auswirkungen diskutiert. Dabei werden die Effekte unterschiedlicher Liquiditätsdimensionen, das Verbot von Leerverkäufen sowie eine im Zeitablauf schwankende Liquidität am Kassamarkt untersucht.

\subsubsection{Basisfall}

Im Rahmen des Modells werden zwei Märkte, ein Kassa- und ein Forwardmarkt, betrachtet. Auf diesen Märkten kann zu Beginn $(t=0)$ und zum Ende $(t=1)$ der ersten und einzigen Periode des Modells gehandelt werden. Des Weiteren wird die Annahme eines risikolosen Zins von $r=0$ getroffen und unterstellt, dass das Kassainstrument keine zwischenzeitliche Zahlungen hat. ${ }^{1}$ Auf dem Terminmarkt agiert, wie in der Studie von Gârleanu et al. (2009), ein repräsentativer Market Maker, der durch eine streng konvexe Nutzenfunktion $u(\cdot)$ beschrieben wird. Dieser Market Maker kann als das Aggregat vieler im Wettbewerb stehender Market Maker angesehen werden. Aufgrund des Wettbewerbs agiert er sowohl auf dem Forward- als auch auf dem Kassamarkt als Preisnehmer. Zum Zeitpunkt $t=0$ gibt es eine exogene Nachfrage nach Forwardkontrakten. Der Market Maker stellt das Angebot an Forwards und nimmt daher die entgegengesetzte Position zur Nachfrage ein. Im Gleichgewicht muss die Nachfrage $H_{N}$ nach Forwardkontrakten dem Angebot $H_{A}$ des Market Makers entsprechen. Folglich gilt im Gleichgewicht: $H_{N} \equiv H_{A}$.

Der Market Maker kann sich gegenüber den Preisrisiken aus seiner Forwardposition durch eine entsprechende Position am Kassamarkt absichern. Die Menge an Kassainstrumenten, die der Market Maker hält, sei mit $X_{0}$ bezeichnet, der fundamentale Preis des Kassainstruments zum Zeitpunkt $t=0$ mit $P_{0}$ und der unsichere fundamentale Preis zum Zeitpunkt $t=1$ mit $\widetilde{P}_{1}$. Durch den Kauf von $X_{0}$ Kassainstrumenten entstehen Transaktionskosten in Höhe von $c\left(X_{0}\right)$. Mit diesen Kosten wird die Illiquidität des Kassamarktes modelliert.

Der Market Maker besitzt ein fixes Anfangsvermögen $W_{0}$ und entscheidet über den Kauf oder Verkauf von $X_{0}$ Kassainstrumenten, wobei Transaktionskosten in Höhe von

\footnotetext{
${ }^{1}$ Die zentralen Aussagen des Modells bestehen bleiben, wenn ein von null verschiedener Zins sowie zwischenzeitliche nicht stochastische Zahlungen des Kassainstruments berücksichtigt werden. Nach Cox et al. (1981) lässt sich das Modell bei nicht stochastischen Zinsen außerdem auf Futures übertragen.
} 
$c\left(X_{0}\right)$ anfallen. Seinen Investitions- oder Finanzierungsbedarf kann er über die risikolose Anleihe $B_{0}$ decken. Sein Vermögen zum Zeitpunkt $t=0$ ist somit gegeben als:

$$
W_{0}=X_{0} P_{0}+c\left(X_{0}\right)+B_{0}
$$

Am Ende der Periode zum Zeitpunkt $t=1$ laufen die Forwardkontrakte aus und der Market Maker stellt seine Position am Kassamarkt glatt. Aus seiner Investition am Kassamarkt erhält er den unsicheren Betrag $X_{0} \widetilde{P}_{1}$ und muss für das Glattstellen dieser Position Transaktionskosten in Höhe von $c\left(-X_{0}\right)$ zahlen. Durch den risikolosen Zins von null, hat die Anleihe zum Zeitpunkt $t=1$ weiterhin einen Wert von $B_{0}$. Darüber hinaus erhält der Market Maker eine unsichere Zahlung aus seiner Forwardposition in Höhe von $-H_{A}\left(\widetilde{P}_{1}-F_{0,1}\right)$, wobei $F_{0,1}$ den Forwardpreis zum Zeitpunkt $t=0$ bezeichnet und der Settlementpreis des Forwards dem fundamentalen Preis $\widetilde{P}_{1}$ entspricht. Sein Endvermögen $\widetilde{W}_{1}$ zum Zeitpunkt $t=1$ ist folglich gegeben durch:

$$
\widetilde{W}_{1}=X_{0} \widetilde{P}_{1}+B_{0}-H_{A}\left(\widetilde{P}_{1}-F_{0,1}\right)-c\left(-X_{0}\right)
$$

Ziel des Market Makers ist es, unter Einhaltung seiner Budgetbeschränkung (3.1) den Erwartungsnutzen seines Endvermögens $\widetilde{W}_{1}$ zum Zeitpunkt $t=1$ gemäß seiner Nutzenfunktion $u(\cdot)$, für die $u^{\prime}(\cdot)>0$ und $u^{\prime \prime}(\cdot)<0$ gilt, was Nichtsättigung und Risikoaversion impliziert, zu maximieren:

$$
\begin{gathered}
\max _{H_{A}, X_{0}} E_{0}\left[u\left(\widetilde{W}_{1}\right)\right], \\
\text { u.d.N. } \quad W_{0}=X_{0} P_{0}+c\left(X_{0}\right)+B_{0} .
\end{gathered}
$$

Um den Erwartungsnutzen seines Vermögens zu maximieren, optimiert der Market Maker über die Menge der Forwardkontrakte $H_{A}$ und Kassainstrumente $X_{0}$ in seinem Portfolio. Die Preisnehmer-Annahme für den Market Maker lässt sich daran erkennen, dass die Menge an Kassainstrumenten sowie die Menge der angebotenen Forwardkontrakte im Portfoliokalkül des Market Makers endogen und abhängig vom Forwardpreis sind. Im Gleichgewicht kommt es zur Markträumung am Forwardmarkt, das heißt die optimale Menge $H_{*}$, welche der Market Maker im Gleichgewicht anbietet, entspricht der Nachfrage nach Forwardkontrakten:

$$
H_{A} \equiv H_{N} \equiv H_{*}
$$


Um bei der Lösung des Portfolioproblems die Budgetbedingung des Market Makers zu berücksichtigen wird sein Anfangsvermögen, Gleichung (3.1), nach $B_{0}$ aufgelöst

$$
W_{0}-X_{0} P_{0}-c\left(X_{0}\right)=B_{0}
$$

und in sein Endvermögen, Gleichung (3.2), eingesetzt. Das Vermögen des Market Makers zum Zeitpunkt $t=1$ kann folglich auch geschrieben werden als:

$$
\widetilde{W}_{1}=W_{0}-X_{0} P_{0}-c\left(X_{0}\right)+X_{0} \widetilde{P}_{1}-c\left(-X_{0}\right)-H_{A}\left(\widetilde{P}_{1}-F_{0,1}\right) .
$$

Damit zeigt sich, dass die Investition des Market Makers in die risikolose Anlage im Rahmen der Erwartungsnutzenmaximierung seines Endvermögens $\widetilde{W}_{1}$ nicht explizit betrachtet werden muss.

Der Forwardpreis kann für jede in der Finanzwirtschaft übliche Form der Nutzenfunktion und unabhängig von der unterstellten Form der Transaktionskosten bestimmt werden. Dazu werden die First-Order-Conditions (FOC) gebildet und dabei Differentiation und Erwartungswert vertauscht: ${ }^{2}$

$$
\begin{aligned}
\frac{\partial E_{0}\left[u\left(\widetilde{W}_{1}\right)\right]}{\partial X_{0}} & =E_{0}\left[u^{\prime}\left(\widetilde{W}_{1}\right)\left(\widetilde{P}_{1}-P_{0}-c^{\prime}\left(X_{0}\right)+c^{\prime}\left(-X_{0}\right)\right)\right] \\
& =E_{0}\left[u^{\prime}\left(\widetilde{W}_{1}\right) \widetilde{P}_{1}\right]-E_{0}\left[u^{\prime}\left(\widetilde{W}_{1}\right)\left(P_{0}+c^{\prime}\left(X_{0}\right)-c^{\prime}\left(-X_{0}\right)\right)\right] \stackrel{!}{=} 0 \\
\frac{\partial E_{0}\left[u\left(\widetilde{W}_{1}\right)\right]}{\partial H_{A}} & =E_{0}\left[u^{\prime}\left(\widetilde{W}_{1}\right)\left(-\left(\widetilde{P}_{1}-F_{0,1}\right)\right)\right] \\
& =-E_{0}\left[u^{\prime}\left(\widetilde{W}_{1}\right) \widetilde{P}_{1}\right]+E_{0}\left[u^{\prime}\left(\widetilde{W}_{1}\right) F_{0,1}\right] \stackrel{!}{=} 0 .
\end{aligned}
$$

Durch das Addieren der Gleichungen (3.6) und (3.7) ergibt sich der Forwardpreis als Preis des Kassainstruments zuzüglich der Kosten, welche aus der Illiquidität des Kassainstruments resultieren:

$$
F_{0,1, *}=P_{0}+c^{\prime}\left(X_{0, *}\right)-c^{\prime}\left(-X_{0, *}\right) .
$$

Für den Spezialfall perfekter Liquidität auf dem Kassamarkt gilt $c\left(X_{0}\right)=0$ und der Forwardpreis entspricht dem Preis, welcher aus dem klassischen Cost-of-Carry-Modell resultiert.

\footnotetext{
${ }^{2}$ Für die genauen Anforderungen an die Nutzenfunktion, welche das Vertauschen von Differentiation und Erwartungswert erlauben, siehe z. B. Elstrodt (2005).
} 
Ziel ist es, anhand eines einfachen Modells Hypothesen zur Liquiditätsübertragung vom Kassa- auf den Forwardmarkt abzuleiten. Dazu ist es nötig das Modell zu lösen. Zur Bestimmung der endogenen Gleichgewichtsgröße $X_{0, *}$ müssen drei Annahmen spezifiziert werden, da $X_{0, *}$ von der Nutzenfunktion des Market Makers, dem Preis des Kassainstruments und den Transaktionskosten abhängt. Um die Hypothesen im Rahmen einer analytischen Lösung ableiten zu können, werden die folgenden Annahmen getroffen:

i) Der Market Maker besitzt die Nutzenfunktion $u\left(\widetilde{W}_{1}\right)=-\frac{1}{A} \exp \left(-A \widetilde{W}_{1}\right)$.

ii) Der Preis des Kassainstruments ist normalverteilt mit $\widetilde{P}_{1} \sim \mathcal{N}\left(P_{0}+\mu, \sigma\right)$.

iii) Die Transaktionskosten haben die Form $c\left(X_{0}\right)=b X_{0}^{2}$.

Nach Annahme (i) besitzt der Market Maker eine konstante absolute Risikoaversion (CARA) A. Zur Modellierung von Investoren ist die CARA-Nutzenfunktion eine Standardklasse in der Finanzwirtschaft, die es in Kombination mit Annahme (ii) ermöglicht eine analytische Lösung des Modells zu finden. ${ }^{3}$ Mit den in Annahme (iii) spezifizierten Transaktionskosten wird unterstellt, dass ein lineares Orderbuch existiert und der Preis des Kassainstruments linear mit der gekauften (verkauften) Menge steigt (fällt). Im Basisfall wird folglich die Liquiditätsdimension der Markttiefe modelliert. Der Illiquiditätsparameter $b$ kann auch als Tiefeparameter bezeichnet werden. Die Dimension der Marktenge konvergiert für eine marginale Einheit gegen null. Daher wird im folgenden Abschnitt die Marktenge durch die Berücksichtigung einer Geld-Brief-Spanne explizit modelliert und analysiert wie dies die Ergebnisse beeinflusst. Die Transaktionskosten zum Zeitpunkt $t=0$ und $t=1$ sind identisch, womit die Dimension der Erneuerungskraft als perfekt unterstellt wird. Verschiedene Studien zeigen, dass die Kassamarktliquidität nach einem großen Auftrag bereits innerhalb weniger Minuten zu ihrem ursprünglichen Niveau zurückkehrt (vgl. z. B. Gomber et al. (2015) für den deutschen Aktienmarkt). Da sich das Kalkül des Market Makers auf einen Zeitraum von einem Tag bis hin zu mehreren Wochen bezieht, ist die Annahme perfekter Erneuerungskraft plausibel.

Mit den Annahmen (i) - (iii) kann das Maximierungsproblem (3.3) analytisch gelöst und die optimale Menge des Market Makers an Kassainstrumenten in geschlossener Form angegeben werden. Der zu maximierende Erwartungsnutzen des Market Makers

\footnotetext{
${ }^{3}$ Es ist bekannt, dass aufgrund der unterstellten Normalverteilung negative Preise eine positive Wahrscheinlichkeit besitzen. Diese Verteilung wird wie in der finanzwissenschaftlichen Literatur üblich als Approximation für die Verteilung der Preise verwendet.
} 
ist unter Annahme einer CARA-Nutzenfunktion gegeben als:

$$
E_{0}\left[u\left(\widetilde{W}_{1}\right)\right]=E_{0}\left[-\frac{1}{A} \exp \left(-A \widetilde{W}_{1}\right)\right]
$$

Der Market Maker ist im Basisfall lediglich dem Preisrisiko ausgesetzt und der Preis des Kassainstruments ist normalverteilt, daher ist das Endvermögen $\widetilde{W}_{1}$ zum Zeitpunkt $t=1$ ebenfalls normalverteilt. Ist eine Zufallsvariable $Y$ normalverteilt mit Erwartungswert $\mu$ und Standardabweichung $\sigma$, dann gilt für die momenterzeugende Funktion ${ }^{4}$

$$
M_{Y}(t)=\exp \left(\mu+\frac{\sigma}{2}\right)
$$

Nach Gleichung (3.10) kann der Erwartungsnutzen daher umgeformt werden zu

$$
\begin{aligned}
& E_{0}\left[-\frac{1}{A} \exp \left(-A \widetilde{W}_{1}\right)\right] \\
= & -\frac{1}{A} \exp \left(-A E_{0}\left[\widetilde{W}_{1}\right]+\frac{1}{2} A^{2} \operatorname{Var}_{0}\left[\widetilde{W}_{1}\right]\right) .
\end{aligned}
$$

Wird Gleichung (3.11) der monoton steigenden Transformation $f(x)=-\frac{1}{A} \log (-A x)$ unterzogen, bleibt das Maximum des Erwartungsnutzen unverändert und es ergibt sich:

$$
E_{0}\left[\widetilde{W}_{1}\right]-\frac{A}{2} \operatorname{Var}_{0}\left[\widetilde{W}_{1}\right]
$$

Das Maximierungsproblem (3.3) ist folglich äquivalent zu

$$
\max _{H_{A}, X_{0}} E_{0}\left[\widetilde{W}_{1}\right]-\frac{1}{2} A \operatorname{Var}_{0}\left[\widetilde{W}_{1}\right]
$$

Unter den Annahmen (i) - (iii) kann der Erwartungswert und die Varianz des Vermögens des Market Makers anhand von Gleichung (3.5) berechnet werden:

$$
E_{0}\left[\widetilde{W}_{1}\right]=W_{0}+\mu X_{0}-2 b X_{0}^{2}-H_{A}\left(P_{0}+\mu-F_{0,1}\right)
$$

und

$$
\operatorname{Var}_{0}\left[\widetilde{W}_{1}\right]=\left(X_{0}-H_{A}\right)^{2} \sigma^{2}
$$

Im Gleichgewicht müssen die FOC und die Markträumungsbedingung (3.4) erfüllt sein.

\footnotetext{
${ }^{4}$ Die momenterzeugende Funktion einer Zufallsvariablen $Y$ ist definiert als $M_{Y}(t):=E\left[e^{t Y}\right]$.
} 
Mit den Momenten (3.13) und (3.14) lässt sich die FOC nach $X_{0}$ schreiben als

$$
\begin{aligned}
\frac{\partial\left(E_{0}\left[\widetilde{W}_{1}\right]-\frac{1}{2} A V a r_{0}\left[\widetilde{W}_{1}\right]\right)}{\partial X_{0}} \stackrel{!}{=} 0 \\
\Leftrightarrow \mu-4 b X_{0}-A\left(X_{0}-H_{A}\right) \sigma^{2}=0 .
\end{aligned}
$$

Wird Gleichung (3.15) nach $X_{0}$ aufgelöst, ergibt sich unter Berücksichtigung der Markträumungsbedingung (3.4) die optimale Menge $X_{0, *}$ an Kassainstrumenten, welche der Market Maker im Gleichgewicht hält:

$$
X_{0, *}=\underbrace{\frac{\mu}{4 b+A \sigma^{2}}}_{\text {spekulative Komponente }}+\underbrace{H_{*} \frac{A \sigma^{2}}{4 b+A \sigma^{2}}}_{\text {Hedgingkomponente }} .
$$

Die optimale Kassamarktposition des Market Makers setzt sich aus zwei Komponenten zusammen. Die erste Komponente ist von der erwarteten Preisänderung im Kassainstrument abhängig und ist folglich spekulativer Art. Die zweite Komponente ist von der nachgefragten Menge an Forwards abhängig und dient dem Market Maker dazu, sich gegenüber Preisveränderungen in seiner Forwardposition abzusichern. Sie wird daher im Folgenden als Hedgingkomponente bezeichnet.

Die spekulative Komponente ist positiv, sofern der Market Maker eine positive Rendite des Kassainstruments erwartet. Je höher die erwartete Rendite, umso größer ist die spekulative Komponente und damit die Kassamarktposition des Market Makers. Diesem Effekt wirken steigende Kosten durch die Illiquidität am Kassamarkt entgegen, da diese Kosten eine Spekulation mit dem Kassainstrument unattraktiver werden lassen. Je höher die Illiquidität am Kassamarkt, desto geringer ist die spekulative Komponente. Den gleichen Effekt bewirken eine steigende Risikoaversion des Market Makers und eine höhere Volatilität des Kassainstruments. Die Faktoren Risikoaversion und Volatilität treten immer gemeinsam auf. Deshalb wird das Produkt der beiden im Folgenden als Risiko am Kassamarkt bezeichnet.

Die Hedgingkomponente ist positiv (negativ), sofern es eine Nachfrage nach Longpositionen (Shortpositionen) am Terminmarkt $H_{*}>0\left(H_{*}<0\right)$ gibt. Besteht eine Nachfrage nach Longpositionen (Shortpositionen) am Terminmarkt, geht der Market Maker eine Shortpositionen (Longpositionen) in Forwards ein und kauft (verkauft) Kassainstrumente, um sich gegen Preisveränderungen in seiner Forwardmarktposition abzusichern. 
Die Höhe der Hedgingkomponente ist von der Illiquidität sowie dem Risiko am Kassamarkt abhängig. Je höher die Illiquidität am Kassamarkt und damit die zu zahlenden Transaktionskosten, desto weniger hedgt der Market Maker. Für den extremen Fall unendlich hoher Transaktionskosten geht die Hedgingkomponente des Market Makers gegen null. In diesem Fall ist es für den Market Maker aufgrund der hohen Transaktionskosten nicht sinnvoll sich gegenüber Preisveränderungen abzusichern. Das Risiko am Kassamarkt wirkt in die entgegengesetzte Richtung. Steigt das Risiko, hedgt der Market Maker mehr, da er eine offene Position hat und risikoavers ist. Geht das Produkt aus Risikoaversion und Volatilität gegen Unendlich, strebt die Hedgingkomponente gegen $H_{*}$, das heißt der Market Maker versucht sich vollständig gegen die Risiken aus seiner Forwardmarktposition abzusichern. Ist der Market Maker dagegen risikoneutral oder die Forwardmarktposition selbst risikolos, ist die Hedgingkomponente gleich null.

Wird die optimale Menge an Kassainstrumenten des Market Makers in Gleichung (3.8) eingesetzt, kann eine Bewertungsformel für den Forwardpreis in Abhängigkeit der Nachfrage angegeben werden:

$$
F_{0,1, *}\left(H_{*}\right)=P_{0}+4 b\left[\frac{\mu}{4 b+A \sigma^{2}}+H_{*} \frac{A \sigma^{2}}{4 b+A \sigma^{2}}\right] .
$$

Die Gleichung zeigt, dass der Forwardpreis neben der Nachfrage von allen übrigen Modellparametern abhängig ist. Eine Nachfrage nach Forward-Longpositionen $\left(H_{*}>0\right)$ erhöht den Forwardpreis. Wie stark der Einfluss der Nachfrage auf den Forwardpreis ist, hängt von der Illiquidität und dem Risiko am Kassamarkt ab.

Abbildung 3.1 veranschaulicht die dem Modell zugrunde liegende Vorstellung der Orderbücher am Kassa- und Forwardmarkt. Für das Orderbuch am Kassamarkt wird der Preis des Kassainstruments gegen die Menge an nachgefragten Kassainstrumenten abgetragen, $P\left(X_{0}\right)=P_{0}+2 b X_{0}$. Die Form des Orderbuchs am Terminmarkt ergibt sich aus der Gleichung (3.17) für den Forwardpreis. Die Form der Transaktionskosten im Basisfall impliziert ein lineares Orderbuch am Kassa- und Forwardmarkt. Je größer die Steigung des Orderbuches, desto sensitiver reagiert der Preis auf Änderungen in der Nachfrage. Ein Forward wird als illiquide bezeichnet, umso sensitiver sein Preis auf Veränderungen in der Nachfrage reagiert. In Abbildung 3.1 sind zwei verschiedene Szenarien dargestellt. Im ersten Szenario erhöht sich die Illiquidität am Kassamarkt. Anhand der Steigung des Orderbuchs am Forwardmarkt zeigt sich bereits, dass sich die Illiquidität nicht eins zu eins überträgt, sondern es zu einem größeren Anstieg der Illiquidität am Forwardmarkt 
Abbildung 3.1: Orderbücher Kassa- und Forwardmarkt: Basisfall
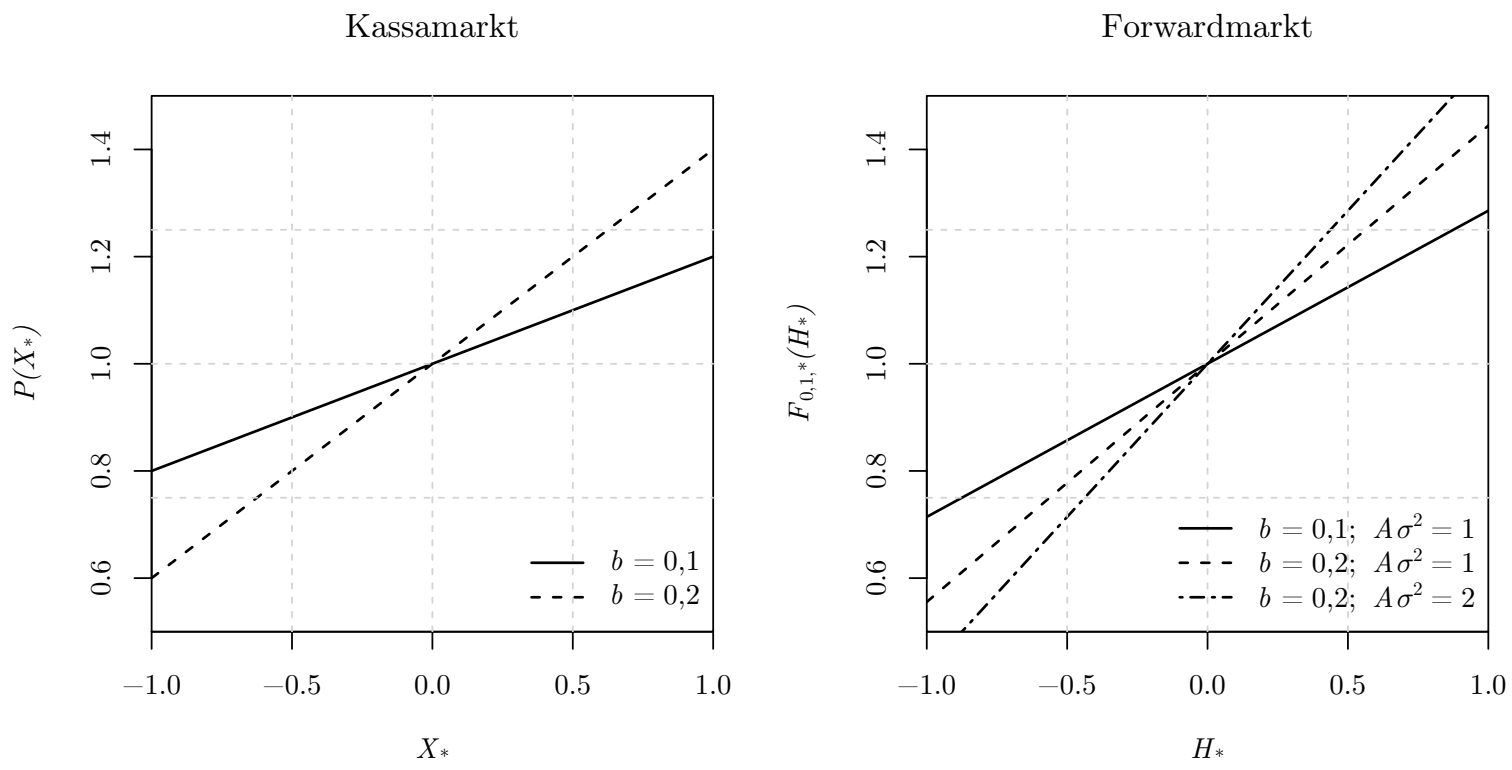

Diese Abbildung zeigt die Preiseinflussfunktionen des Kassa- und Forwardmarktes $\left(P\left(X_{*}\right)\right.$ und $\left.F_{0,1, *}\left(H_{*}\right)\right)$ für unterschiedliche Parameter der Kassamarktliquidität $(b)$ und des Risikos am Kassamarkt $\left(A \sigma^{2}\right)$. Ausgangspunkt bilden die Preiseinflussfunktionen für die Parameter $P_{0}=1, \mu=0$, $\sigma^{2}=0,25, A=4$ und $b=0,1$.

kommt. Im zweiten Szenario steigt sowohl Illiquidität als auch das Risiko am Kassamarkt, was einen noch größeren Anstieg der Illiquidität am Forwardmarkt zur Folge hat.

Aufgrund der Beziehung von Forwardpreis und Nachfrage in der Bewertungsgleichung (3.17) kann der Einfluss der Modellparameter auf die Illiquidität am Forwardmarkt genauer studiert werden. Gleichung (3.17) dient daher als Grundlage für das Illiquiditätsmaß $\operatorname{ILM}\left(H_{*}\right)$, das wie folgt definiert wird:

$$
\operatorname{ILM}\left(H_{*}\right)=\frac{F_{0,1, *}\left(H_{*}\right)-F_{0,1, *}(0)}{H_{*}} .
$$

$\operatorname{ILM}\left(H_{*}\right)$ misst den Preisunterschied, der sich bei einer Nachfrage von $H_{*}$ und einer Nachfrage von 0 ergeben würde, pro Nachfrage von $H_{*}$. Es nimmt sein Minimum von null an, wenn der Forwardmarkt perfekt liquide ist. Das $I L M\left(H_{*}\right)$ ist konzeptionell äquivalent zu dem Preiseinflussparameter $\lambda$ von Kyle (1985). Mit dem Illiquiditätsmaß kann 
insbesondere die Rolle der Kassamarktliquidität für den Forwardmarkt analysiert werden sowie welche weiteren Faktoren die Transmission der Kassamarktliquidität beeinflussen.

Für den Fall eines illiquiden Kassamarktes mit $b \neq 0$, ergibt sich ein $\operatorname{ILM}\left(H_{*}\right)$ in Höhe von:

$$
\operatorname{ILM}\left(H_{*}\right)=\frac{4 b A \sigma^{2}}{4 b+A \sigma^{2}} .
$$

Die Illiquidität des Terminmarktes hängt damit lediglich von den zwei Größen Illiquidität und Risiko am Kassamarkt ab. Je größer diese beiden Faktoren sind, desto größer ist die Illiquidität des Terminmarktes. Dabei zeigt sich, dass sich die Liquidität des Kassamarktes nicht eins zu eins überträgt, sondern in Verbindung mit der Volatilität des Kassainstruments und der Risikoaversion des Market Makers steht.

Im Spezialfall eines perfekt liquiden Kassamarktes $(b=0)$ nimmt das $\operatorname{ILM}\left(H_{*}\right)$ den Wert von null an. Ist der Kassamarkt perfekt liquide kommt es folglich zu keiner Illiquiditätsübertragung und somit ist der Terminmarkt ebenfalls perfekt liquide. Würde der Gleichgewichtspreis vom Cost-of-Carry-Preis abweichen, könnte der Market Maker einen risikolosen Gewinn (Verlust) machen. Dies wiederspricht jedoch der Annahme eines im Wettbewerb stehenden Market Makers. Bei perfekter Liquidität auf dem Kassamarkt nimmt der Market Maker die Rolle des Arbitrageurs aus der klassischen No-Arbitrage Bewertung ein.

Zusammenfassend lässt sich festhalten, dass sowohl eine steigende Illiquidität als auch ein steigendes Risiko am Kassamarkt zu einer höheren Illiquidität am Terminmarkt führen. Bereits mit der einfachen Modellierung von nicht perfekter Markttiefe in Form eines linearen Orderbuches lassen sich folglich zwei empirisch testbare Hypothesen zur Illiquiditätsübertragung ableiten:

HL1: Je höher die Illiquidität am Kassamarkt, desto höher die Illiquidität am Forwardmarkt.

HL2: Je höher das Risiko am Kassamarkt, desto höher die Illiquidität am Forwardmarkt.

Die Bewertungsgleichung (3.17) ermöglicht es darüber hinaus Hypothesen über den Forwardpreis abzuleiten. Diese Preishypothesen unterscheiden sich nicht von denen, die anhand des Mehrperiodenmodells abgeleitet werden. Da das Mehrperiodenmodell zusätzlich die explizite Modellierung von kurz- und langfristigen Kontrakten sowie die 
Analyse der Preiseffekte für diese Kontrakte ermöglicht, werden die Preishypothesen geschlossen im Rahmen des Mehrperiodenmodells diskutiert. In den folgenden Abschnitten wird zunächst die Liquiditätsübertragung anhand verschiedener Modellvarianten genauer studiert.

\subsubsection{Geld-Brief-Spanne am Kassamarkt}

In diesem Abschnitt wird die Annahme eines linearen Orderbuches aufgehoben und die Modellierung der Transaktionskosten am Kassamarkt um eine Liquiditätsdimension erweitert. Zusätzlich zur Tiefekomponente muss nun unabhängig von der Ordergröße bei einem Kauf oder Verkauf von Kassainstrumenten ein fixer Betrag in Höhe der halben Geld-Brief-Spanne gezahlt werden, d.h. es wird explizit die Dimension der Marktenge berücksichtigt. Ziel dieses Kapitels ist es zu analysieren, welche Auswirkungen die Modellierung dieser zusätzlichen Liquiditätsdimension am Kassamarkt auf die Hedgingstrategie des Market Makers, die Preissetzung am Terminmarkt und damit auf die Liquiditätsübertragung hat.

Wird die halbe Geld-Brief-Spanne mit $a$ bezeichnet, fallen für jedes gekaufte oder verkaufte Kassainstrument zusätzliche Kosten in Höhe von $a$ an. Für den Kauf von $X_{0}$ Wertpapieren fallen damit Transaktionskosten in Höhe von

$$
c\left(X_{0}\right)=a\left|X_{0}\right|+b X_{0}^{2}
$$

an. Der erste Summand $a\left|X_{0}\right|$ kann als Engekomponente und der zweite Summand $b X_{0}^{2}$ als Tiefekomponente bezeichnet werden.

Die Lösung des Maximierungsproblems (3.3) mit Transaktionskosten in Form von Gleichung (3.20) verläuft analog zum Basisfall. Jedoch muss beim Bilden der FOC nach $X_{0}$ aufgrund der Betragsfunktion in den Transaktionskosten eine Fallunterscheidung getroffen werden. Es sind drei Fälle zu unterscheiden: Der Market Maker geht eine Longposition $X_{0, *}>0$, eine Shortposition $X_{0, *}<0$ oder keine Position am Kassamarkt $X_{0, *}=0$ ein. Für diese Fälle ergibt sich die optimale Kassamarktposition des Market Makers als:

$$
X_{0, *}=\left\{\begin{array}{ccc}
\frac{-2 a}{4 b+A \sigma^{2}}+\frac{\mu}{4 b+A \sigma^{2}}+H_{*} \frac{A \sigma^{2}}{4 b+A \sigma^{2}} & X_{0, *}>0 \\
\frac{2 a}{4 b+A \sigma^{2}}+\frac{\mu}{4 b+A \sigma^{2}}+H_{*} \frac{A \sigma^{2}}{4 b+A \sigma^{2}} & \text { für } \quad & X_{0, *}<0 \\
0 & \text { sonst. }
\end{array}\right.
$$


Für den Fall einer Longposition (Shortposition) wird die optimale Menge des Market Makers um den Betrag $\frac{2 a}{4 b+A \sigma^{2}}$ reduziert (erhöht). Die zusätzlichen Kosten in Höhe von $a$ führen folglich dazu, dass der Market Maker sich im Optimum weniger stark am Kassamarkt engagiert. Dieser Effekt ist umso schwächer, je größer der Tiefeparameter $b$ und das Kassamarktrisiko ist. Im Falle eines hohen Risikos am Kassamarkt, ist der Market Maker bereit die höheren Kosten für die Absicherung seiner Forwardposition zu zahlen. Die beiden anderen Terme sind für den Fall einer Long- oder Shortposition des Market Makers am Kassamarkt identisch mit denen des Basisfalls. An der nicht differenzierbaren Stelle der Transaktionskostenfunktion ist die optimale Menge des Market Makers gleich null, das heißt er geht keine Position am Kassamarkt ein.

Zur Bestimmung der Bewertungsgleichungen für den Forwardpreis wird zunächst die FOC nach $H_{A}$ gebildet

$$
\begin{aligned}
\frac{\partial\left(E_{0}\left[\widetilde{W}_{1}\right]-\frac{1}{2} A \operatorname{Var}_{0}\left[\widetilde{W}_{1}\right]\right)}{\partial H_{A}} & \stackrel{!}{=} 0 \\
\Leftrightarrow-P_{0}-\mu+F_{0,1}+A\left(X_{0}-H_{A}\right) \sigma^{2} & =0,
\end{aligned}
$$

die optimale Menge $X_{0, *}$ in Gleichung (3.22) eingesetzt und nach $F_{0,1}$ aufgelöst. Daraus ergeben sich wiederum drei Fälle für den Forwardpreis. Um anzugeben welche Forwardpreisgleichung für eine bestimmte Nachfrage an Forwardkontrakten gültig ist, wird in die Ungleichungen aus (3.21) die optimale Menge $X_{0, *}$ eingesetzt und nach $H_{*}$ aufgelöst. Damit sind die Forwardpreisgleichungen gegeben durch:

$$
F_{0,1, *}\left(H_{*}\right)=\left\{\begin{array}{ccc}
P_{0}+\frac{4 b \mu}{4 b+A \sigma^{2}}-a \frac{2 A \sigma^{2}}{4 b+A \sigma^{2}}+H_{*} \frac{4 b A \sigma^{2}}{4 b+A \sigma^{2}} & H_{*}>\frac{2 a-\mu}{A \sigma^{2}} \\
P_{0}+\frac{4 b \mu}{4 b+A \sigma^{2}}+a \frac{2 A \sigma^{2}}{4 b+A \sigma^{2}}+H_{*} \frac{4 b A \sigma^{2}}{4 b+A \sigma^{2}} & \text { für } & H_{*}<-\frac{2 a+\mu}{A \sigma^{2}} \\
P_{0}+\mu+A \sigma^{2} H_{*} & \text { sonst. }
\end{array}\right.
$$

Zur Veranschaulichung der Orderbücher am Kassa- und Terminmarkt wird erneut der Preis für ein Kassainstrument gegen die gekaufte Menge an Kassainstrumenten abgetragen und ebenso der Preis für einen Forward gegen die angebotene Menge an Forwards. Abbildung 3.2 zeigt, dass das Orderbuch am Terminmarkt keine konstante Steigung aufweist. Der Forwardpreis reagiert damit auf Änderungen in der Nachfrage unterschiedlich sensitiv. Die Symmetrie um den Punkt $(0,1)$ ist nur für den Fall von $\mu=0$ gegeben. Wie sich an Gleichung (3.23) nachvollziehen lässt, verschiebt eine positive (negative) erwartete Rendite die Preiseinflussfunktion $F_{0,1, *}\left(H_{*}\right)$ nach links (rechts). 
Abbildung 3.2: Orderbücher Kassa- und Forwardmarkt: Geld-Brief-Spanne

Kassamarkt

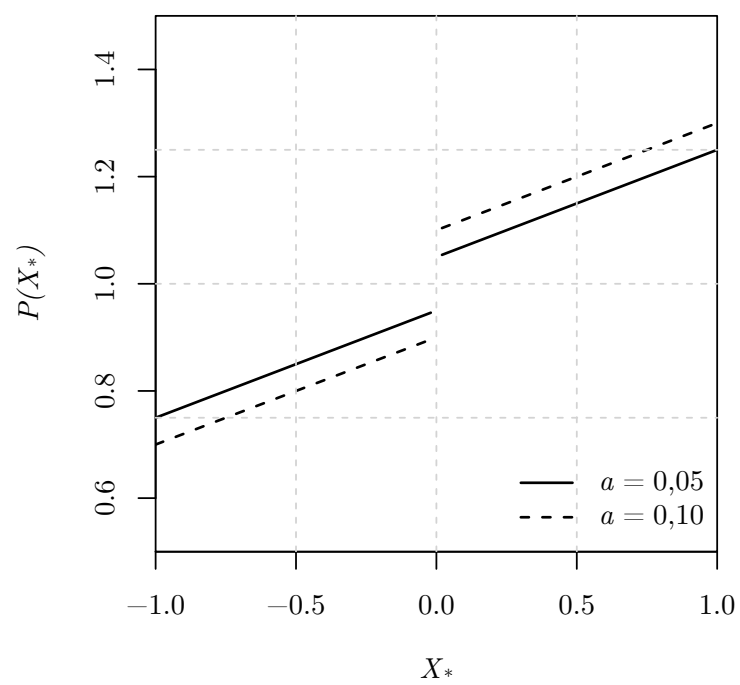

Forwardmarkt

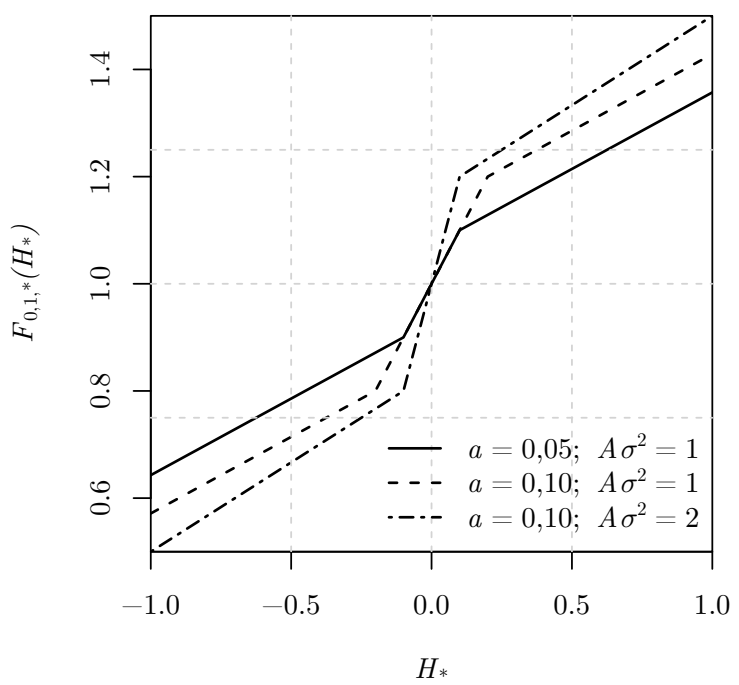

Diese Abbildung zeigt die Preiseinflussfunktionen des Kassa- und Forwardmarktes $\left(P\left(X_{*}\right)\right.$ und $\left.F_{0,1, *}\left(H_{*}\right)\right)$ für unterschiedliche Parameter der halben Geld-Brief-Spanne am Kassamarkt $(a)$ und des Risikos am Kassamarkt $\left(A \sigma^{2}\right)$. Ausgangspunkt bilden die Preiseinflussfunktionen für die Parameter $P_{0}=1, \mu=0, \sigma^{2}=0,25, A=4, a=0,05$ und $b=0,1$.

Um das Phänomen unterschiedlicher Steigungen genauer zu analysieren, werden analog zum Basisfall die Illiquiditätsmaße aus den Forwardgleichungen für die entsprechenden Fälle abgeleitet:

$$
I L M\left(H_{*}\right)=\left\{\begin{array}{ccc}
A \sigma^{2} & \text { für } & -\frac{2 a+\mu}{A \sigma^{2}}<H_{*}<\frac{2 a-\mu}{A \sigma^{2}} \\
\frac{4 b A \sigma^{2}}{4 b+A \sigma^{2}} & & \text { sonst. }
\end{array}\right.
$$

Anhand des $I L M\left(H_{*}\right)$ zeigt sich, dass die Illiquiditätsübertragung vom Kassa- auf den Terminmarkt identisch zum Basisfall ist, sofern die Nachfrage nach Forwardpositionen größer als $\frac{2 a-\mu}{A \sigma^{2}}$ oder kleiner als $-\frac{2 a+\mu}{A \sigma^{2}}$ ist. Liegt die Forwardnachfrage innerhalb dieses Intervalls, hedgt sich der Market Maker nicht. In diesem Bereich geht der Market Maker aufgrund der zusätzlichen Kosten am Kassamarkt keine Position im Kassainstrument ein wie sich anhand der Gleichungen (3.21) und (3.23) nachvollziehen lässt. Dies führt dazu, dass sich das Risiko, dem der Market Maker ausgesetzt ist, direkt auf den Terminmarkt überträgt. Der Forwardpreis reagiert in diesem Fall besonders sensitiv auf Veränderungen 
in der Nachfrage, dass heißt der Terminmarkt ist in diesem Fall besonders illiquide. Dies zeigt sich auch an der höheren Steigung in der Orderbuchgrafik.

Die Größe des Intervalls, in dem der Market Maker sich nicht absichert, ist von der GeldBrief-Spanne, dem Kassamarktrisiko und der erwarteten Rendite abhängig. Je größer die Geld-Brief-Spanne, umso größer ist der Bereich in dem sich der Market Maker nicht absichert. Diesem Effekt wirkt ein steigendes Risiko am Kassamarkt entgegen. Das Risiko wirkt auf zwei Arten. Zum einen verstärkt sich durch ein erhöhtes Risiko die Illiquidität am Terminmarkt allgemein und besonders stark im Bereich in dem der Market Maker nicht hedgt. Zum anderen wird dieser Bereich, in dem der Market Maker sich hedgt, durch ein höheres Risiko kleiner, da es aufgrund des gestiegenen Risikos für den Market Maker optimal ist sich trotz der zusätzlichen Kosten abzusichern.

Der Extremfall einer Geld-Brief-Spanne, die gegen Unendlich geht, ist in den Auswirkungen vergleichbar mit einem Tiefeparameter $b$, der gegen Unendlich strebt. Wie sich anhand von Gleichung (3.24) nachvollziehen lässt sind die Kosten für die Absicherung über den Kassamarkt in beiden Fällen so hoch, dass der Kassamarkt nicht zum Hedgen genutzt werden kann.

Die erwartete Rendite hat keine Auswirkungen auf die Größe des Intervalls, sondern verschiebt es lediglich entlang der Achse für die Forwardnachfrage. Das $\operatorname{ILM}\left(H_{*}\right)$ ist in allen Fällen unabhängig von der Geld-Brief-Spanne. Der Einfluss der Geld-Brief-Spanne auf die Liquiditätsübertragung beschränkt sich damit darauf, dass durch sie festgelegt wird bei welcher Nachfrage der Market Maker keine Position am Kassamarkt eingeht.

\subsubsection{Asymmetrische Preiseinflussfunktion und Leerverkaufsverbote}

Die Annahme bezüglich der Transaktionskosten am Kassamarkt im Basisfall impliziert, dass die Kosten für Käufe und Leerverkäufe von Kassainstrumenten identisch sind. Im folgenden Abschnitt wird diese Annahmen aufgehoben und der Basisfall dahingehend erweitert, dass die Transaktionskosten aus einem asymmetrischen Orderbuch resultieren. Der Fokus liegt dabei auf der Tiefe des Orderbuches und für den Engeparameter gilt $a=0$.

Aufgrund der Asymmetrie des Orderbuches existieren zwei unterschiedliche Tiefeparameter. Der erste Tiefeparameter ist gültig für Longpositionen $\left(b_{l}\right)$ und der zweite für 
Shortpositionen $\left(b_{s}\right)$ am Kassamarkt. Dabei wird die Annahme getroffen, dass der Preiseinfluss am Kassamarkt stärker im Falle eines Leerverkaufes ist, $b_{s}>b_{l}$. Der Market Maker steht folglich höheren Kosten gegenüber, wenn er eine Shortposition eingeht. Die Transaktionskosten am Kassamarkt haben damit folgende Form:

$$
c\left(X_{0}\right)=\left\{\begin{array}{lll}
b_{l} X_{0}^{2} & \text { für } \quad \begin{array}{l}
X_{0} \geq 0 \\
b_{s} X_{0}^{2}
\end{array} & X_{0}<0 .
\end{array}\right.
$$

Diese Form der Transaktionskosten verändert das Hedgingverhalten des Market Makers in der Weise, dass seine optimale Kassamarktposition nun ebenfalls davon abhängt, ob eine Long- oder Shortposition zur Absicherung benötigt wird. Das Vorgehen zur Lösung des Portfolioproblems des Market Makers verläuft wie im Basisfall mit dem Unterschied, dass beim Bilden der FOC nach $X_{0}$ aufgrund der asymmetrischen Preiseinflussfunktion am Kassamarkt eine Fallunterscheidung getroffen werden muss. Die optimale Menge an Kassainstrumenten ergibt sich damit als:

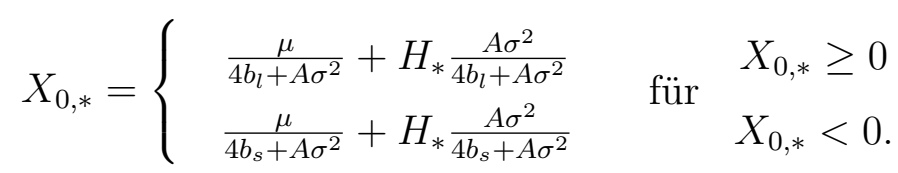

Durch die höheren Transaktionskosten für eine Shortposition fällt die optimale Menge des Market Makers in diesem Fall kleiner aus als die optimale Menge im Fall einer Longposition. Die Wirkung der Parameter auf die optimale Menge am Kassamarkt bleibt identisch zum Basisfall. Wird diese optimale Menge in die Gleichung (3.22) eingesetzt, können die Bewertungsgleichungen für den Forwardpreis gefunden werden: ${ }^{5}$

$$
F_{0,1, *}\left(H_{*}\right)=\left\{\begin{array}{l}
P_{0}+4 b_{l}\left[\frac{\mu}{4 b_{l}+A \sigma^{2}}+H_{*} \frac{A \sigma^{2}}{4 b_{l}+A \sigma^{2}}\right] \\
P_{0}+4 b_{s}\left[\frac{\mu}{4 b_{s}+A \sigma^{2}}+H_{*} \frac{A \sigma^{2}}{4 b_{s}+A \sigma^{2}}\right]
\end{array} \quad \text { für } \begin{array}{l}
H_{*} \geq-\frac{\mu}{A \sigma^{2}} \\
H_{*}<-\frac{\mu}{A \sigma^{2}} .
\end{array}\right.
$$

Die Abbildung 3.3 liefert eine anschauliche Darstellung der Preiseinflussfunktionen am Kassa- und Forwardmarkt. Im ersten Szenario wird deutlich, dass es durch das asymmetrische Orderbuch am Kassamarkt auch auf dem Forwardmarkt zu asymmetrischen Effekten kommt. Im zweiten Szenario können die Auswirkungen einer positiven erwarteten Rendite auf das Hedgingverhalten des Market Makers nachvollzogen werden. Die positive erwartete Rendite führt dazu, dass der Market Maker trotz einer Nachfrage

${ }^{5}$ Die Umformung der Ungleichungen erfolgt wie in Abschnitt 3.1.2. 
Abbildung 3.3: Orderbücher Kassa- und Forwardmarkt: Asymmetrische Preiseinflussfunktion
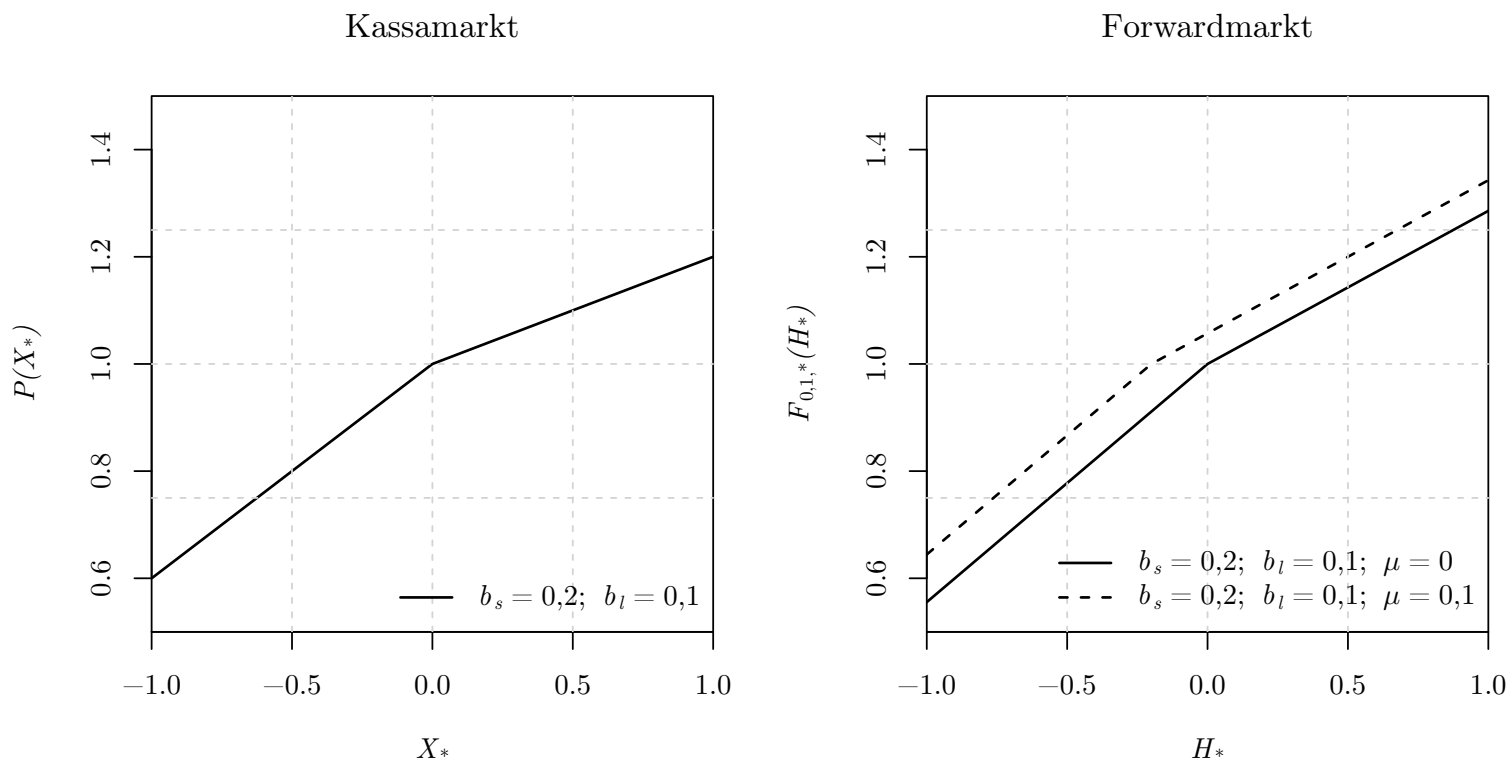

Diese Abbildung zeigt die Preiseinflussfunktionen des Kassa- und Forwardmarktes $\left(P\left(X_{*}\right)\right.$ und $\left.F_{0,1, *}\left(H_{*}\right)\right)$ für unterschiedliche Parameter der Kassamarktliquidität $\left(b_{l}\right.$ und $\left.b_{s}\right)$ und der erwarteten Rendite am Kassamarkt $(\mu)$. Ausgangspunkt bilden die Preiseinflussfunktionen für die Parameter $P_{0}=1$, $\mu=0, \sigma^{2}=0,25, A=4$ sowie $b_{l}=0,1$ und $b_{s}=0,2$.

nach Forward-Shortpositionen selbst eine Longposition im Kassainstrument hält. Je höher die erwartete Rendite, umso größer muss die Nachfrage nach Shortpositionen sein, damit der Market Maker trotz der positiven erwarteten Rendite eine Shortposition zur Absicherung am Kassamarkt eingeht. Dem Effekt einer positiven erwarteten Rendite wirkt die Volatilität des Kassainstruments sowie die Risikoaversion des Market Makers entgegen. Die Berechnung des $I L M\left(H_{*}\right)$ ergibt:

$$
\operatorname{ILM}\left(H_{*}\right)=\left\{\begin{array}{ccl}
\frac{4 b_{l} A \sigma^{2}}{4 b_{l}+A \sigma^{2}} \\
\frac{4 b_{s} A \sigma^{2}}{4 b_{s}+A \sigma^{2}} & \text { für } \quad \begin{array}{l}
H_{*} \geq-\frac{\mu}{A \sigma^{2}} \\
\end{array} \quad H_{*}<-\frac{\mu}{A \sigma^{2}} .
\end{array}\right.
$$

Anhand des $\operatorname{ILM}\left(H_{*}\right)$ lässt sich erkennen, dass bei höheren Transaktionskosten für Shortpositionen auch der Forwardpreis für Shortpositionen sensitiver auf Änderungen in der Nachfrage als bei Käufen reagiert, da $\frac{4 b_{l} A \sigma^{2}}{4 b_{l}+A \sigma^{2}}<\frac{4 b_{s} A \sigma^{2}}{4 b_{s}+A \sigma^{2}}$.

Der Extremfall unendlich hoher Transaktionskosten für Leerverkäufe am Kassamarkt ist 
Abbildung 3.4: Orderbücher Kassa- und Forwardmarkt: Leerverkaufsverbote
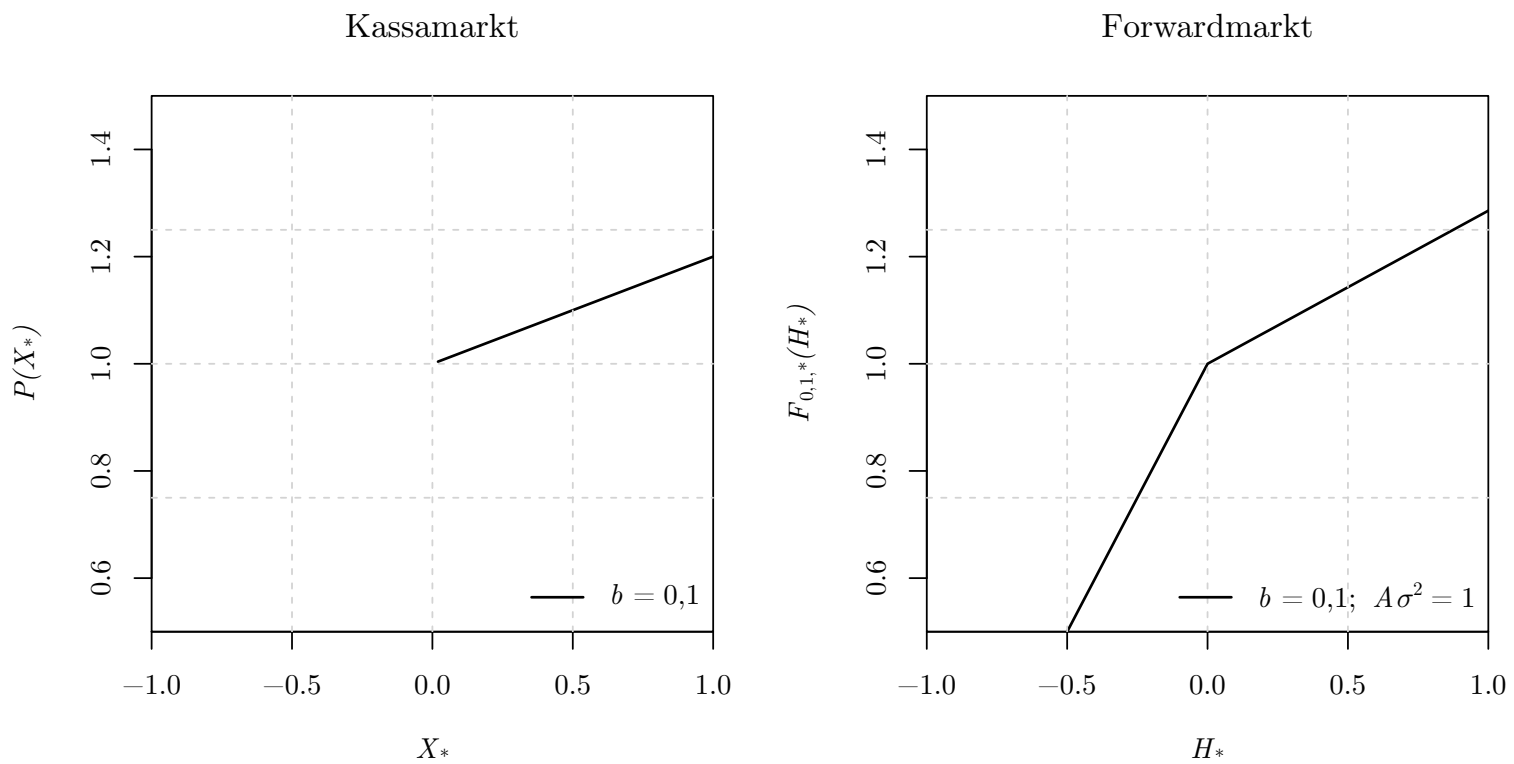

Diese Abbildung zeigt die Preiseinflussfunktionen des Kassa- und Forwardmarktes $\left(P\left(X_{*}\right)\right.$ und $\left.F_{0,1, *}\left(H_{*}\right)\right)$ bei einem Verbot von Leerverkäufen. Die Parameter der Preiseinflussfunktionen sind $P_{0}=1$, $\mu=0, \sigma^{2}=0,25, A=4$ und $b=0,1$.

vergleichbar mit einem Verbot von Leerverkäufen. In diesem Spezialfall stehen dem Market Maker Leerverkäufe zur Absicherung seiner Forwardposition nicht zur Verfügung. Da der Market Maker weiterhin verpflichtet ist die Nachfrage nach Shortpositionen am Forwardmarkt zu bedienen, die entsprechende Gegenposition jedoch nicht absichern kann, wird sich das Risiko, dem der Market Maker ausgesetzt ist, direkt auf die Illiquidität am Forwardmarkt auswirken. Abbildung 3.4 zeigt, dass der Forwardpreis wesentlich sensitiver auf Veränderungen in der Nachfrage nach Shortpositionen als auf Veränderungen in der Nachfrage nach Longpositionen reagiert.

\subsubsection{Liquiditätsrisiko am Kassamarkt}

In der Realität schwankt die Liquidität und damit die Transaktionskosten am Kassamarkt im Zeitablauf. Um diesem Phänomen Rechnung zu tragen, wird die Annahme aufgehoben, dass die Transaktionskosten über die Zeit konstant sind. Schwankende Transaktionskosten bedeuten für das Modell, dass der Market Maker die Transaktionskosten lediglich für den Zeitpunkt $t=0$ kennt. Die Transaktionskosten beim Glattstellen 
seiner Kassamarktposition in $t=1$ sind jedoch unbekannt. Die zum Zeitpunkt $t=1$ unbekannten Transaktionskosten stellen für den Market Maker ein zusätzliches Risiko dar. Dieses Risiko kann als Liquiditätsrisiko interpretiert werden.

Zum Zeitpunkt $t=0$ wird weiterhin von einem linearen Orderbuch mit bekannter Steigung $b_{0}$ ausgegangen. Die Steigung $\widetilde{b}_{1}$ zum Zeitpunkt $t=1$ ist dagegen stochastisch. Der Market Maker ist nun zwei Risiken ausgesetzt: dem Preisrisiko $\left(\widetilde{P}_{1}\right)$ sowie dem Liquiditätsrisiko $\left(\widetilde{b}_{1}\right)$ am Kassamarkt. Dabei wird angenommen, dass die zwei Zufallsvariablen eine gemeinsame Normalverteilung haben:

$$
\left(\begin{array}{c}
\widetilde{P}_{1} \\
\tilde{b}_{1}
\end{array}\right) \sim \mathcal{N}\left[\left(\begin{array}{c}
P_{0}+\mu \\
b_{0}
\end{array}\right)\left(\begin{array}{cc}
\sigma^{2} & \sigma \eta \rho \\
\sigma \eta \rho & \eta^{2}
\end{array}\right)\right],
$$

wobei mit $\eta$ der Volatilitätsparameter für das Liquiditätsrisiko und mit $\rho$ der Korrelationskoeffizient der Zufallsvariablen $\widetilde{P}_{1}$ und $\widetilde{b}_{1}$ bezeichnet wird. Damit ist das Vermögen des Market Makers zum Zeitpunkt $t=1$ gegeben als:

$$
\widetilde{W}_{1}=W_{0}-P_{0} X_{0}-b_{0} X_{0}^{2}+\widetilde{P}_{1} X_{0}-\tilde{b}_{1} X_{0}^{2}-H_{A}\left(\widetilde{P}_{1}-F_{0,1}\right) .
$$

Unter der Annahme eines normalverteilten Liquiditätsrisikos kann gezeigt werden, dass eine eindeutige reelle Lösung für die optimale Menge an Kassainstrumenten $X_{0, *}$ existiert. Die Herleitung der geschlossenen Formel für $X_{0, *}$ sowie des Forwardpreises $F_{0,1, *}\left(H_{*}\right)$ erfolgt im Anhang A.1. Es zeigt sich, dass unter Berücksichtigung des Liquiditätsrisikos zwischen der optimalen Menge $X_{0, *}$ und der Nachfrage $H_{*}$ eine nichtlineare Beziehung besteht. Der Forwardpreis $F_{0,1, *}\left(H_{*}\right)$ ergibt sich als:

$$
F_{0,1, *}\left(H_{*}\right)=P_{0}+4 b_{0} X_{0, *}+\frac{1}{2} A\left[3 X_{0, *}^{3} \eta^{2}-2\left(X_{0, *}^{2}-X_{0, *} H_{*}\right) \sigma \eta \rho\right]
$$

Gleichung (3.26) zeigt, dass der Forwardpreis wie im Basisfall von allen Modellparametern abhängig ist.

Im Folgenden werden die Auswirkungen der verschiedenen Modellparameter auf die Illiquidität des Forwardmarkets analysiert. Wird die Forwardpreisgleichung (3.26) unter Verwendung der optimalen Kassamarktposition $X_{0, *}$ in Gleichung (3.18) eingesetzt, ergibt sich die geschlossene Formel für das $I L M\left(H_{*}\right)$ anhand derer komparativ statische Grafiken erstellt werden. Ausgangspunkt bildet jeweils das $\operatorname{ILM}\left(H_{*}\right)$, das für die $\mathrm{Pa}$ rameter $\mu=0,05, \sigma=0,3, A=4, b=0,5 \%, \eta=0,2 \%$ und $\rho=0$ berechnet 
wird. ${ }^{6}$ Darauf aufbauend werden einzelne Parameter verändert, um deren Einfluss auf die Illiquidität am Forwardmarkt zu untersuchen.

Zunächst wird untersucht, ob die Effekte für die aktuelle Kassamarktliquidität $b_{0}$, Volatilität $\sigma$ und Risikoaversion $A$ im Fall mit Liquiditätsrisiko mit denen des Basisfalls vergleichbar sind. Panel A, B und C in Abbildung 3.5 zeigen die Resultate für komparativ statische Veränderungen der Parameter $b_{0}, \sigma$ und $A$. Diese Grafiken bestätigen die Resultate aus dem Basisfall qualitativ. Eine steigende Illiquidität, eine steigende Volatilität und eine steigende Risikoaversion führen zu einer größeren Illiquidität am Forwardmarkt. Darüber hinaus zeigt sich, dass die Illiquidität am Forwardmarkt von der Nachfrage abhängt und die Illiquidität mit einer höheren absoluten Nachfrage ansteigt. Der Illiquiditätseffekt ist für die Volatilität und Risikoaversion ausgeprägter für große absolute Werte von $H_{*}$. Für die Illiquidität des Kassamarktes zeigt sich der Effekt bereits bei kleinen Mengen deutlich.

Panel D zeigt wie ein steigendes Liquiditätsrisiko die Illiquidität am Forwardmarkt beeinflusst. Auch ein höheres Liquiditätsrisiko wirkt sich positiv auf die Illiquidität des Forwardmarktes aus. Dieser Effekt ist intuitiv, da der Market Maker einem höheren Risiko ausgesetzt ist. Der Effekt zeigt sich ebenfalls besonders stark für hohe absolute Werte der Nachfrage. Für sehr kleine Werte von $H_{*}$ kommt es dagegen nur zu minimalen Effekten. Damit kann eine weitere empirische zu überprüfende Hypothese zu den Liquiditätseffekten abgeleitet werden:

\section{HL3: Je höher das Liquiditätsrisiko am Kassamarkt, desto höher die Illiquidität am For- wardmarkt.}

Eine weitere Frage ist, wie sich Veränderungen in der Korrelation zwischen Preis- und Liquiditätsrisiko sowie der erwarteten Rendite auf die Illiquidität am Forwardmarkt auswirken. Panel A und B in Abbildung 3.6 zeigen die Resultate für komparativ statische Veränderungen der Parameter $\rho$ und $\mu$. Die beiden Effekte unterscheiden sich von den vorherigen dadurch, dass die Wirkungen asymmetrisch auftreten, das heißt, es kommt zu unterschiedlichen Effekten, wenn die Werte von $H_{*}$ größer oder kleiner null sind.

Bei einer Forwardnachfrage nach Longpositionen $\left(H_{*}>0\right)$ ist die Illiquidität hoch, sofern die Korrelation positiv ist. Besteht dagegen eine Forwardnachfrage nach Shortpositionen

\footnotetext{
${ }^{6}$ Die Parameter für erwartete Rendite, Standardabweichung, Illiquidität und Illiquiditätsrisiko des Kassainstruments sind vergleichbar mit den Werten der DAX30 Aktien, die in der anschließenden empirischen Analyse verwendet werden. Korn et al. (2015) zeigen, dass die Ergebnisse für alternative Parameterkonstellationen qualitativ unverändert bleiben.
} 
Abbildung 3.5: Illiquidität am Forwardmarkt: Effekte von Kassamarktliquidität, Risikoaversion, Kassamarktvolatilität und Liquiditätsrisiko

Panel A

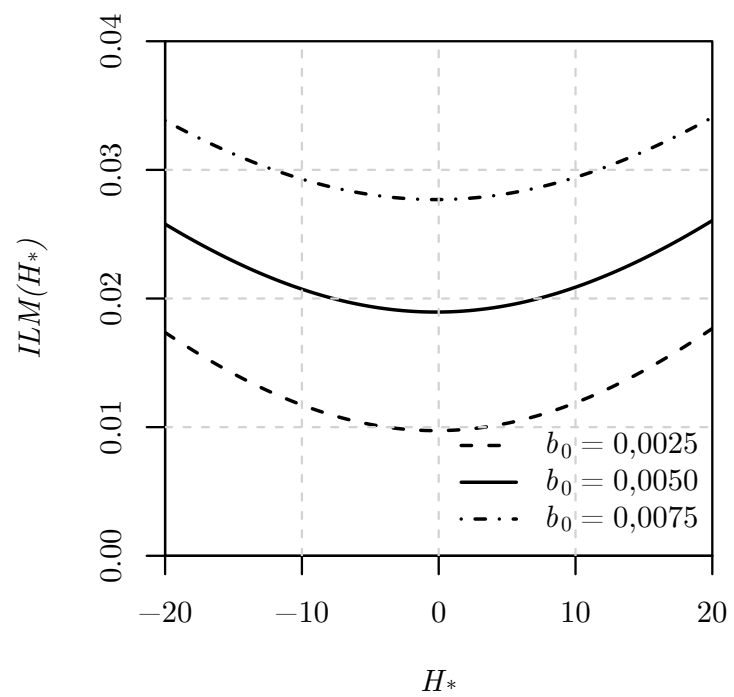

Panel C

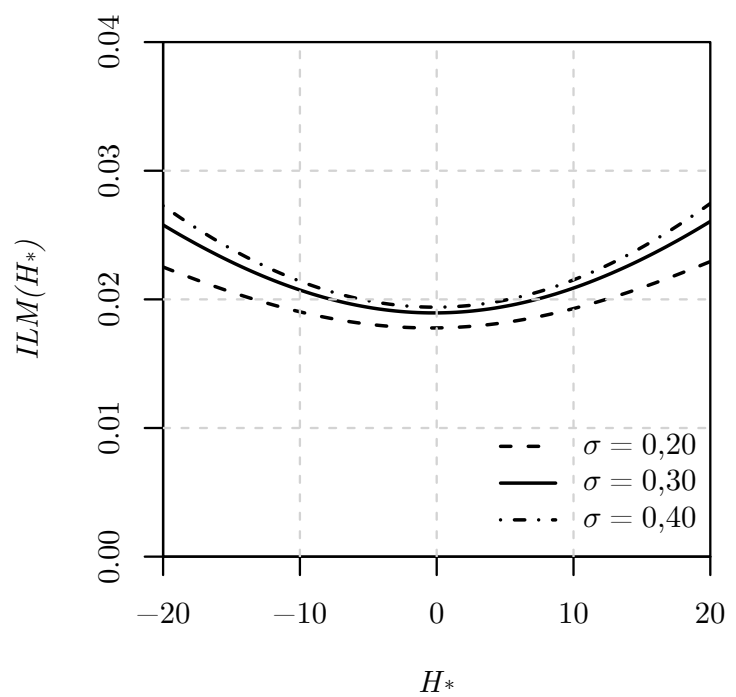

Panel B

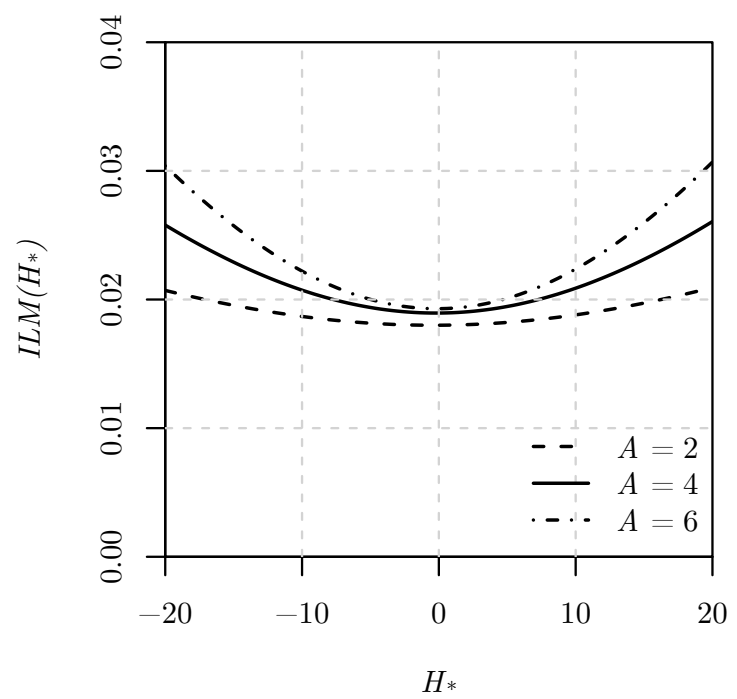

Panel D

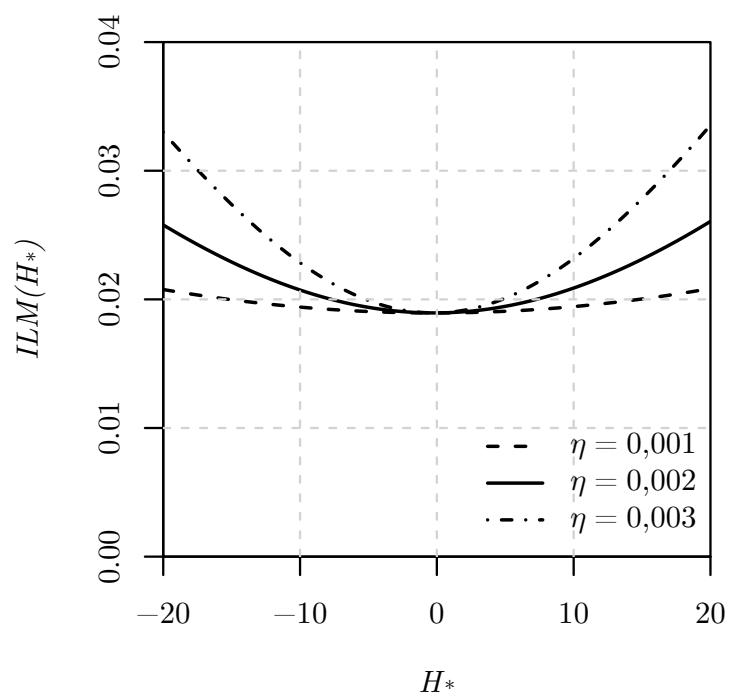

Diese Abbildung zeigt das $I L M\left(H_{*}\right)$ für unterschiedliche Parameter der Kassamarktliquidität ( $b_{0}$, Panel A), der Risikoaversion des Market Makers ( $A$, Panel B), der Kassamarktvolatilität ( $\sigma$, Panel C) und des Liquiditätsrisikos ( $\eta$, Panel D). Ausgangspunkt bildet jeweils das $I L M\left(H_{*}\right)$ für die Parameter $\mu=0,05$, $\sigma=0,3, A=4, b_{0}=0,5 \%, \eta=0,2 \%$ und $\rho=0$. 
Abbildung 3.6: Illiquidität am Forwardmarkt: Effekte der Korrelation von Preis- und Liquiditätsrisiko sowie der erwarteten Rendite

Panel A

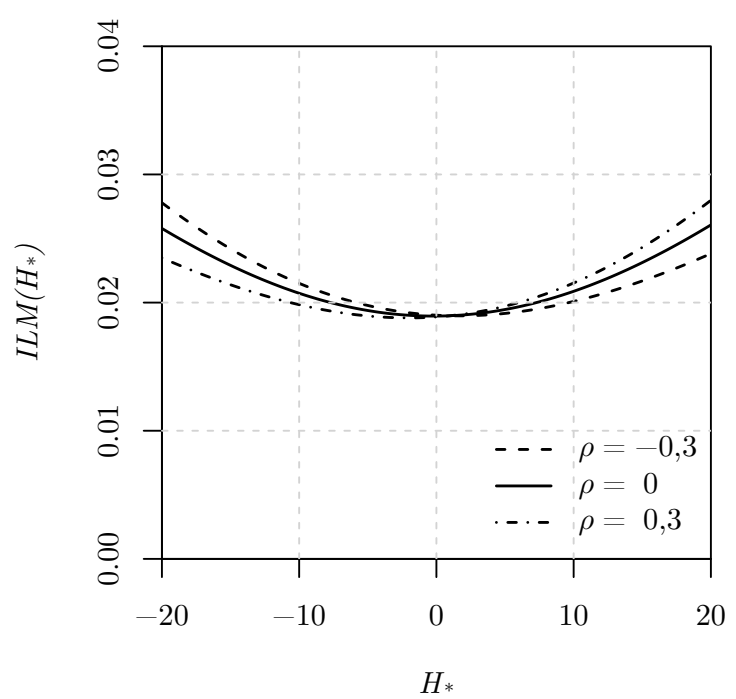

Panel B

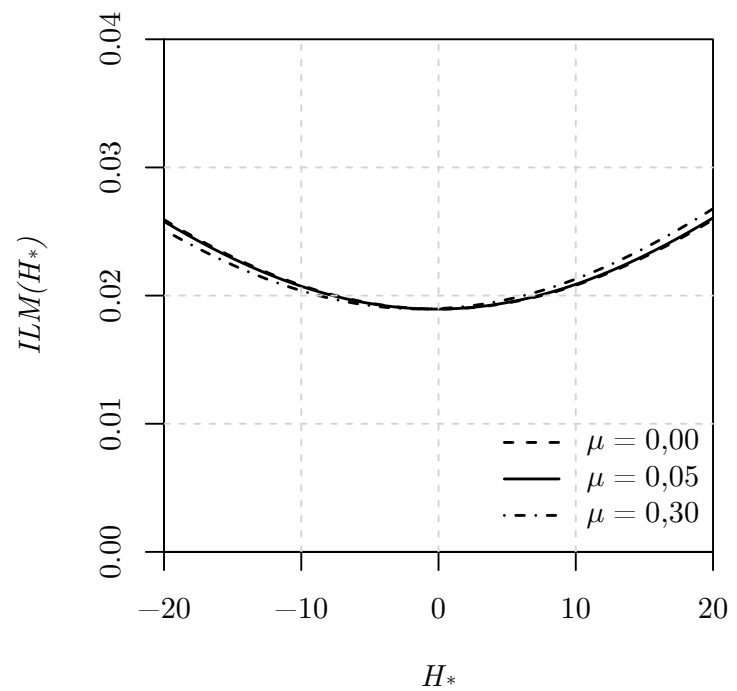

Diese Abbildung zeigt das $I L M\left(H_{*}\right)$ für unterschiedliche Parameter der Korrelation von Preis- und Liquiditätsrisiko ( $\rho$, Panel A), sowie der erwarteten Rendite am Kassamarkt ( $\mu$, Panel B). Ausgangspunkt bildet jeweils das $\operatorname{ILM}\left(H_{*}\right)$ für die Parameter $\mu=0,05, \sigma=0,3, A=4, b_{0}=0,5 \%, \eta=0,2 \%$ und $\rho=0$.

$\left(H_{*}<0\right)$ ist der entgegengesetzte Effekt zu beobachten. Intuitiv kann dieser asymmetrische Effekt wie folgt erläutert werden: Bei einer Nachfrage nach Longpositionen am Forwardmarkt geht der Market Maker die entgegengesetzte Position am Forwardmarkt ein, um die Nachfrage zu bedienen und ist damit short am Forwardmarkt. Diese Shortposition sichert er wiederum mit einer Longposition am Kassamarkt ab. Jedoch sichert sich der Market Marker aufgrund der Illiquidität am Kassamarkt nicht vollständig ab. ${ }^{7}$ Folglich hält der Market Maker eine Netto-Shortposition. Steigt der Preis am Kassamarkt, verliert die Gesamtposition des Market Makers an Wert. Ist der Preis am Kassamarkt außerdem positiv mit der Illiquidität am Kassamarkt korreliert, ist es wahrscheinlich, dass es neben dem Preisanstieg am Kassamarkt zu einem Anstieg der Illiquidität am Kassamarkt kommt. In diesem Fall verliert der Market Maker doppelt: Zum einen auf-

${ }^{7}$ Ist die erwartete Rendite am Kassamarkt ungleich null, wird der Market Maker zusätzlich zu seiner Hedgingposition eine spekulative Position eingehen. Die Schlussfolgerungen bzgl. der Illiquiditätstransmissionen sind unabhängig von der spekulativen Position des Market Markers. Dies kann gezeigt werden, indem das Modell für $\mu=0$ gelöst wird. 
grund des Preisanstiegs am Kassamarkt und zum anderen durch die aufgrund der höheren Illiquidität gestiegenen Transaktionskosten beim Glattstellen seiner Position. Für dieses Risiko muss der risikoaverse Market Maker kompensiert werden, was zu einem illiquideren Forwardmarkt führt. Durch ein vergleichbares Argument lässt sich der weniger illiquide Forwardmarkt im Fall einer negativen Korrelation erklären. Die negative Korrelation von Preis- und Liquiditätsrisiko generiert eine Art natürlichen Hedge und reduziert dadurch das Gesamtrisiko des Market Makers. Folglich steigt die Illiquidität des Forwardmarktes bei positiven Werten für $H_{*}$ mit steigendem $\rho$. Der umgekehrte Effekt stellt sich für negative Werte von $H_{*}$ ein, da der Market Maker in diesem Fall eine Netto-Longposition hält. In diesem Fall sinkt die Illiquidität des Forwardmarktes für steigendes $\rho$.

Zur Erklärung des asymmetrischen Effekts für die erwartete Rendite, wird erneut von einer Nachfrage nach Longpositionen am Forwardmarkt ausgegangen, was bedeutet, dass der Market Maker eine Netto-Shortposition hält. Eine hohe positive erwartete Rendite bedeutet in diesem Fall für den Market Maker, dass das Hedging teuer ist und mit steigender erwarteter Rendite weniger attraktiv wird. Daher verlangt der Market Maker eine Kompensation in Form höherer Forwardpreise, was wiederum zu höherer Illiquidität für $H_{*}>0$ führt. Besteht dagegen eine Nachfrage nach Shortpositionen, hält der Market Maker eine Netto-Longposition. Eine steigende erwartete Rendite lässt diese Position profitabler werden, wodurch der Market Maker eine geringere Kompensation benötigt, was den umgekehrten Effekt eines liquideren Forwardmarktes erklärt. Die ökonomische Bedeutung der erwarteten Rendite ist jedoch gering.

Im Rahmen des Einperiodenmodells konnten bereits wesentliche Treiber für die Illiquidität am Forwardmarkt identifiziert werden. Die Illiquidität, das Liquiditätsrisiko und die Volatilität des Kassainstruments sowie die Risikoaversion des Market Makers stehen in einem positiven Zusammenhang zur Illiquidität am Forwardmarkt. Die Erweiterung um das Liquiditätsrisiko zeigt darüber hinaus, dass insbesondere die Einflüsse der letzten drei Variablen stärker ausgeprägt für eine große absolute Nachfrage nach Forwardkontrakten sind. Im folgenden Abschnitt soll mit der Restlaufzeit der Forwardkontrakte ein weiterer wesentlicher Treiber für die Illiquidität am Forwardmarkt analysiert werden. Dazu ist es nötig den Basisfall des Modells um eine Periode zu erweitern und ein dynamisches Modell zu entwickeln. 


\subsection{Mehrperiodenmodell}

In diesem Abschnitt wird zunächst die Struktur des Mehrperiodenmodells erläutert. Durch die Erweiterung des Einperiodenmodells um eine zusätzliche Periode hat der Market Maker die Möglichkeit sich dynamisch am Kassamarkt abzusichern. Es werden die optimalen Kassamarktpositionen des Market Makers bestimmt und untersucht, ob der Market Maker die Option der dynamischen Absicherung nutzt. Darüber hinaus werden sowohl die Preis- als auch die Liquiditätseffekte von Forwards mit unterschiedlicher Laufzeit analysiert.

\subsubsection{Modellstruktur}

Auf dem Kassa- und Forwardmarkt kann zu Beginn $(t=0)$, zum Ende der ersten $(t=1)$ und zum Ende der zweiten Periode $(t=2)$ gehandelt werden. Auf dem Forwardmarkt werden Kontrakte mit Verfallszeitpunkt in $t=1$ und Verfallszeitpunkt in $t=2$ gehandelt. Zum Zeitpunkt $t=0$ gibt es eine exogene Nachfrage $H_{0,1, N}$ nach Forwardkontrakten mit Verfallszeitpunkt in $t=1$. Diese Kontrakte haben einen Preis von $F_{0,1}$, einen Settlementpreis von $\widetilde{P}_{1}$ und eine Laufzeit von $t=0$ bis $t=1$. Darüber hinaus gibt es ebenfalls zum Zeitpunkt $t=0$ eine exogene Nachfrage $H_{0,2, N}$ nach Forwardkontrakten mit Verfallszeitpunkt in $t=2$. Diese Kontrakte haben einen Preis von $F_{0,2}$, einen Settlementpreis von $\widetilde{P}_{2}$ und eine Laufzeit von $t=0$ bis $t=2$.

Wie im Einperiodenmodell agiert auch im Mehrperiodenmodell ein repräsentativer Market Maker auf dem Forwardmarkt. Dieser Market Maker stellt das Angebot an Forwardkontrakten mit Verfallszeitpunkt in $t=1$ und $t=2\left(H_{0,1, A}\right.$ und $\left.H_{0,2, A}\right)$ und sichert seine Position über den Kassamarkt ab. Im Gleichgewicht muss das Angebot des Market Makers der Nachfrage nach Forwardkontrakten entsprechen:

$$
H_{0,1, N} \equiv H_{0,1, A} \equiv H_{0,1, *} \quad \text { und } \quad H_{0,2, N} \equiv H_{0,2, A} \equiv H_{0,2, *} .
$$

Auch zum Zeitpunkt $t=1$ muss im Gleichgewicht die Nachfrage nach Kontrakten mit Verfallszeitpunkt in $t=2\left(H_{1,2, N}\right)$ dem Angebot nach Kontrakten mit Verfallszeitpunkt in $t=2\left(H_{1,2, A}\right)$ entsprechen:

$$
H_{1,2, N} \equiv H_{1,2, A} \equiv H_{1,2, *} .
$$


Zum Zeitpunkt $t=1$ gibt es keine Änderungen in der Nachfrage nach Forwardkontrakten mit Verfallszeitpunkt in $t=2$. Folglich besteht zum Zeitpunkt $t=1$ die gleiche Nachfrage nach Forwardkontrakten mit Verfallszeitpunkt in $t=2$ wie zum Zeitpunkt $t=0$ und es gilt:

$$
H_{1,2, N}=H_{0,2, N}
$$

Das Ziel des Market Makers im Mehrperiodenmodell ist es sein Endvermögen $\widetilde{W}_{2}$ zu maximieren. Zum Zeitpunkt $t=0$ besitzt der Market Maker wie im Basisfall ein fixes Vermögen in Höhe von $W_{0}$ und geht eine Position in $X_{0}$ Kassainstrumenten ein, für die er Transaktionskosten in Höhe von $b X_{0}^{2}$ zahlt. In $t=1$ hat diese Kassamarktposition aus Sicht von $t=0$ einen Wert von $X_{0}\left(\widetilde{P}_{1}-P_{0}\right)$. Aus seiner Investition in Forwards mit Verfallszeitpunkt in $t=1$ erhält der Market Maker den aus Sicht von $t=0$ unsicheren Betrag in Höhe von $-H_{0,1, A}\left(\widetilde{P}_{1}-F_{0,1}\right)$. Seine Postition in Forwards mit Verfallszeitpunkt in $t=2$ ist zum Zeitpunkt $t=1$ aus Sicht von $t=0$ gegeben durch $-H_{0,2, A}\left(\widetilde{F}_{1,2}-\right.$ $F_{0,2}$ ), wobei $\widetilde{F}_{1,2}$ den unsicheren Preis des Forwards mit Verfallszeitpunkt in $t=2$ zum Zeitpunkt $t=1$ bezeichnet. Aufgrund des risikolosen Zins von $r=0$ ergibt sich das Vermögen $\widetilde{W}_{1}$ des Market Makers zum Zeitpunkt $t=1$ aus Sicht von $t=0$ als:

$$
\widetilde{W}_{1}=W_{0}+X_{0}\left(\widetilde{P}_{1}-P_{0}\right)-b X_{0}^{2}-H_{0,1, A}\left(\widetilde{P}_{1}-F_{0,1}\right)-H_{0,2, A}\left(\widetilde{F}_{1,2}-F_{0,2}\right) .
$$

Zum Zeitpunkt $t=1$ ist das Vermögen $W_{1}$ des Market Maker fix und er hat die Möglichkeit seine Position am Kassamarkt anzupassen. Er passt seine Kassamarktposition an, so dass seine neue Kassamarktposition $X_{1}$ optimal ist. Dabei fallen Transaktionskosten in Höhe von $b\left(X_{1}-X_{0}\right)^{2}$ an. Zum Zeitpunkt $t=2$ stellt der Market Maker seine Kassamarktposition glatt und erhält den aus Sicht von $t=1$ unsicheren Betrag $X_{1}\left(\widetilde{P}_{2}-P_{1}\right)$ abzüglich der Transaktionskosten in Höhe von $b\left(-X_{1}\right)^{2}$. Darüber hinaus laufen die Forwardkontrakte mit Verfallszeitpunkt in $t=2$ aus und der Market Maker erhält eine aus Sicht von $t=1$ unsichere Zahlung von $H_{0,2, A}\left(\widetilde{P}_{2}-F_{1,2}\right)$ aus dieser Position. Damit ist das Vermögen $\widetilde{W}_{2}$ des Market Makers zum Zeitpunkt $t=2$ aus Sicht von $t=1$ gegeben durch:

$$
\widetilde{W}_{2}=W_{1}+X_{1}\left(\widetilde{P}_{2}-P_{1}\right)-b\left(X_{1}-X_{0}\right)^{2}-H_{0,2, A}\left(\widetilde{P}_{2}-F_{1,2}\right)-b\left(-X_{1}\right)^{2} .
$$




\subsubsection{Kassamarktpositionen des Market Makers}

Um die optimalen Kassamarktpositionen des Market Makers $X_{0, *}$ und $X_{1, *}$ zu den Zeitpunkten $t=0$ und $t=1 \mathrm{zu}$ bestimmen, muss das Portfolioproblem des Market Makers gelöst werden. Dabei handelt es sich, aufgrund der Option des Market Makers zum Zeitpunkt $t=1$ erneut zu handeln, um ein dynamisches Portfolioproblem, das per Rückwärtsinduktion gelöst werden kann. Das Vorgehen erfolgt dabei in zwei Schritten. Zunächst wird die optimale Kassamarktposition des Market Makers zum Zeitpunkt $t=1$ bestimmt. Dazu wird der Erwartungsnutzen seines Vermögens $E_{1}\left[u\left(\widetilde{W}_{2}\right)\right]$ unter den zum Zeitpunkt $t=1$ verfügbaren Informationen maximiert. Zu diesem Zeitpunkt ist sein Vermögen $W_{1}$ bekannt und es kommt zu keiner neuen Nachfrage nach Forwardkontrakten mit Verfallszeitpunkt in $t=2$. Der Market Maker optimiert daher ein Portfolio, welches lediglich aus $X_{1}$ Wertpapieren und $H_{0,2, A}$ Forwardkontrakten besteht. In einem zweiten Schritt kann mit Hilfe seiner zuvor bestimmten optimalen Kassamarktposition $X_{1, *}$ der Erwartungsnutzen seines Vermögens $E_{0}\left[u\left(\widetilde{W}_{2}\right)\right]$ unter den zum Zeitpunkt $t=0$ verfügbaren Informationen maximiert werden, um seine optimale Kassamarkposition $X_{0, *}$ zum Zeitpunkt $t=0 \mathrm{zu}$ bestimmen.

Der Market Maker wird, wie im Einperiodenmodell, durch eine CARA-Nutzenfunktion beschrieben und die Preise des Kassainstruments $\widetilde{P}_{1}$ und $\widetilde{P}_{2}$ sind unabhängig normalverteilt mit $\widetilde{P}_{1} \sim \mathcal{N}\left(P_{0}+\mu, \sigma\right)$ und $\widetilde{P}_{2} \sim \mathcal{N}\left(P_{1}+\mu, \sigma\right)$. Unter diesen Annahmen ist die Maximierung des Erwartungsnutzens

$$
\max _{H_{0,2, A}, X_{1}} E_{1}\left[u\left(\widetilde{W}_{2}\right)\right]
$$

äquivalent $\mathrm{zu}$

$$
\max _{H_{0,2, A}, X_{1}} E_{1}\left[\widetilde{W}_{2}\right]-\frac{1}{2} A \operatorname{Var}_{1}\left(\widetilde{W}_{2}\right)
$$

Der Erwartungswert des Vermögens $\widetilde{W}_{2}$ unter den Informationen zum Zeitpunkt $t=1$ ist gegeben als

$$
E_{1}\left[\widetilde{W}_{2}\right]=W_{1}+\mu X_{1}-b\left(X_{1}-X_{0}\right)^{2}-\mu H_{0,2, A}-H_{0,2, A}\left(P_{1}-F_{1,2}\right)-b\left(-X_{1}\right)^{2}
$$

und die Varianz als

$$
\operatorname{Var}_{1}\left[\widetilde{W}_{2}\right]=\left(X_{1}-H_{0,2, A}\right)^{2} \sigma^{2}
$$


Mit den Momenten (3.32) und (3.33) lässt sich die FOC nach $X_{1}$ schreiben als

$$
\begin{aligned}
\frac{\partial\left(E_{1}\left[\widetilde{W}_{2}\right]-\frac{1}{2} A \operatorname{Var}_{1}\left[\widetilde{W}_{2}\right]\right)}{\partial X_{1}} & \stackrel{!}{=} 0 \\
\Leftrightarrow \mu-2 b\left(X_{1}-X_{0}\right)+2 b\left(-X_{1}\right)-A\left(X_{1}-H_{0,2, A}\right) \sigma^{2} & =0 .
\end{aligned}
$$

Durch das Auflösen von Gleichung (3.34) nach $X_{1}$ ergibt sich unter Berücksichtigung der Gleichgewichtsbedingung (3.28) sowie der Gleichung (3.29) die Menge, die der Market Maker im Optimum zum Zeitpunkt $t=1$ hält:

$$
X_{1, *}=\frac{\mu+2 b X_{0}}{4 b+A \sigma^{2}}+H_{0,2, *} \frac{A \sigma^{2}}{4 b+A \sigma^{2}} .
$$

Die optimale Menge $X_{1, *}$ besteht aus einer spekulativen Komponente sowie einer Hedgingkomponente, die zur Absicherung gegenüber den Risiken aus der Forwardposition mit Verfallszeitpunkt in $t=2$ dient. Neben diesen Komponenten ist die optimale Menge $X_{1, *}$ außerdem von der zum Zeitpunkt $t=0$ eingegangenen Kassamarktposition $X_{0}$ abhängig. Um diese Position zu bestimmen, wird im zweiten Schritt der Rückwärtsinduktion der Erwartungsnutzen des Endvermögens $E_{0}\left[u\left(W_{2}\right)\right]$ maximiert. Dazu optimiert der Market Maker ein Portfolio bestehend aus Forwardkontrakten mit Verfallszeitpunkt in $t=1$ und $t=2\left(H_{0,1, A}\right.$ und $\left.H_{0,2, A}\right)$ sowie seiner Kassamarktposition $X_{0}$. Die Maximierung des Erwartungsnutzen des Market Makers lässt sich umformen zu:

$$
\max _{H_{0,1, A}, H_{0,2, A}, X_{0}} E_{0}\left[\widetilde{W}_{2}\right]-\frac{1}{2} A \operatorname{Var}_{0}\left(\widetilde{W}_{2}\right)
$$

Nach dem Einsetzen von Gleichung (3.30) in (3.31), lassen sich die ersten beiden Momente des Vermögens $\widetilde{W}_{2}$ schreiben als

$$
\begin{aligned}
E_{0}\left[\widetilde{W}_{2}\right]= & W_{0}+X_{0} \mu-b X_{0}^{2}-H_{0,1, A}\left(\mu+P_{0}-F_{0,1}\right) \\
& -H_{0,2, A}\left(2 \mu+P_{0}-F_{0,2}\right)+X_{1, *} \mu-b\left(X_{1, *}-X_{0}\right)-b\left(-X_{1, *}\right)^{2}
\end{aligned}
$$

und

$$
\operatorname{Var}_{0}\left(\widetilde{W}_{2}\right)=\left(X_{0}-H_{0,1, A}-H_{0,2, A}\right)^{2} \sigma^{2}+\left(X_{1, *}-H_{0,2, A}\right)^{2} \sigma^{2}
$$

Im Optimum müssen die Gleichgewichtsbedingungen (3.27) sowie die FOC erfüllt sein. Beim Bilden der FOC nach $X_{0}$ ist zu beachten, dass die Menge $X_{1, *}$ von $X_{0}$ abhängig 
ist:

$$
\begin{aligned}
\frac{\partial\left(E_{0}\left[\widetilde{W}_{2}\right]-\frac{1}{2} A \operatorname{Var}_{0}\left[\widetilde{W}_{2}\right]\right)}{\partial X_{0}} \stackrel{!}{=} 0 \\
\Leftrightarrow \frac{1}{4 b+A \sigma^{2}}\left[\mu\left(6 b+A \sigma^{2}\right)-X_{0}\left(12 b^{2}+8 b A \sigma^{2}+\left(A \sigma^{2}\right)^{2}\right)\right. \\
\left.+H_{0,1, A}\left(4 b A \sigma^{2}+\left(A \sigma^{2}\right)^{2}\right)+H_{0,2, A}\left(6 b A \sigma^{2}+\left(A \sigma^{2}\right)^{2}\right)\right]=0 .
\end{aligned}
$$

Durch das Auflösen von Gleichung (3.36) nach $X_{0}$ ergibt sich die optimale Kassamarktposition des Market Makers zum Zeitpunkt $t=0$ :

$$
X_{0, *}=\frac{\mu}{2 b+A \sigma^{2}}+H_{0,1, *} \frac{4 b A \sigma^{2}+\left(A \sigma^{2}\right)^{2}}{12 b^{2}+8 b A \sigma^{2}+\left(A \sigma^{2}\right)^{2}}+H_{0,2, *} \frac{A \sigma^{2}}{2 b+A \sigma^{2}} .
$$

Die optimale Kassamarktposition zum Zeitpunkt $t=0$ besteht aus einer spekulativen sowie zwei Hedgingkomponenten. Die erste Hedgingkomponente dient dazu sich gegenüber den Risiken aus der Forwardposition mit Verfallszeitpunkt in $t=1$ abzusichern und die zweite Komponente dazu Risiken aus der Forwardposition mit Verfallszeitpunkt in $t=2$ abzusichern. Wie im Basisfall erhöht eine positive erwartete Rendite die Kassamarktposition des Market Makers. Dieser Effekt wird durch steigende Transaktionskosten und ein steigendes Kassamarktrisiko verringert. Die Auswirkungen der unterschiedlichen Modellparameter auf die beiden Hedgingkomponenten sind ebenfalls vergleichbar mit dem Basisfall. Bei steigendem Kassamarktrisiko hedgt der Market Maker mehr und bei steigenden Transaktionskosten weniger. Beide Hedgingkomponenten streben gegen null bzw. gegen die Nachfrage nach Forwardkontrakten, wenn die Transaktionskosten bzw. das Kassamarktrisiko gegen unendlich streben. Gibt es keine Transaktionskosten werden die Risiken aus den unterschiedlichen Kontrakten voll gehedgt. Sofern kein Kassamarktrisiko existiert, sind beide Hedgingkomponenten gleich null.

Die Kosten der Illiquidität steigen quadratisch mit der gekauften Menge an Kassainstrumenten an. Aufgrund dieser Art der Transaktionskosten ist es möglicherweise für den Market Maker optimal, zunächst weniger zu hedgen und seine Portfolioposition nachträglich aufzustocken. Es ist daher von Interesse zu untersuchen, ob der Market Maker den Zeitpunkt $t=1$ nutzt um seine Portfolioposition anzupassen.

Um die optimale Menge $X_{1, *}$ in Abhängigkeit der exogenen Modellparameter auszu- 
drücken, wird die optimale Menge $X_{0, *}$ in Gleichung (3.35) eingesetzt

$$
\begin{aligned}
X_{1, *} & =\frac{\mu+2 b\left[\frac{\mu}{2 b+A \sigma^{2}}+H_{0,1, *} \frac{4 b A \sigma^{2}+\left(A \sigma^{2}\right)^{2}}{12 b^{2}+8 b A \sigma^{2}+\left(A \sigma^{2}\right)^{2}}+H_{0,2, *} \frac{A \sigma^{2}}{2 b+A \sigma^{2}}\right]}{4 b+A \sigma^{2}}+H_{0,2, *} \frac{A \sigma^{2}}{4 b+A \sigma^{2}} \\
\Leftrightarrow X_{1, *} & =\frac{\mu}{2 b+A \sigma^{2}}+H_{0,1, *} \frac{\left(A \sigma^{2}\right)^{2}+4 b A \sigma^{2}}{\left(A \sigma^{2}\right)^{2}+8 b A \sigma^{2}+12 b^{2}}+H_{0,2, *} \frac{A \sigma^{2}}{2 b+A \sigma^{2}} .
\end{aligned}
$$

Es zeigt sich, dass diese Menge der optimalen Menge für $X_{0, *}$ aus Gleichung (3.37) entspricht und der Market Maker zum Zeitpunkt $t=1$ folglich keinen weiteren Handel betreibt. Um zu untersuchen, ob sich seine Kassamarktposition im Rahmen des dynamischen Modells von seiner Kassamarktposition im Einperiodenmodell unterscheidet wird angenommen, dass es keine Nachfrage nach Forwardkontrakten mit Verfallszeitpunkt in $t=1$ gibt $\left(H_{0,1, N}=0\right)$. Unter dieser Annahme vereinfacht sich Gleichung (3.37) für die optimale Menge des Market Makers zu:

$$
X_{0, *}=\frac{\mu}{2 b+A \sigma^{2}}+H_{0,2, *} \frac{A \sigma^{2}}{2 b+A \sigma^{2}}
$$

Im Basisfall wirkt die Illiquidität doppelt so stark. Die Kassamarktposition (3.38) ist jedoch identisch zur Kassamarktposition im Basisfall (3.16), wenn dafür korrigiert wird, dass der Market Maker sich im dynamischen Modell über zwei Perioden absichert. Die Möglichkeit sich dynamisch abzusichern hat folglich keine Auswirkungen auf die Wahl der Kassamarktposition zu Beginn der ersten Periode.

\subsubsection{Preiseffekte}

Zur Analyse der Preiseffekte müssen zunächst die Preise der Forwardkontrakte mit Verfallszeitpunkt in $t=1$ und $t=2$ bestimmt werden. Im Optimum müssen die Gleichgewichtsbedingungen (3.27) gelten sowie die FOC. Die FOC nach $H_{0,1, A}$ und $H_{0,2, A}$ sind gegeben durch:

$$
\begin{aligned}
& \frac{\partial E_{0}\left[u\left(\widetilde{W}_{2}\right)\right]}{\partial H_{0,1, A}} \stackrel{!}{=} 0 \\
& \Leftrightarrow F_{0,1}-P_{0}-\mu+A\left(X_{0}-H_{0,1, A}-H_{0,2, A}\right) \sigma^{2}=0
\end{aligned}
$$


und

$$
\begin{gathered}
\frac{\partial E_{0}\left[u\left(\widetilde{W}_{2}\right)\right]}{\partial H_{0,2, A}} \stackrel{!}{=} 0 \\
\Leftrightarrow-\frac{1}{4 b+A \sigma^{2}}\left[-F_{0,2}\left(4 b+A \sigma^{2}\right)+P_{0}\left(4 b+A \sigma^{2}\right)\right. \\
+\mu\left(8 b+A \sigma^{2}\right)-X_{0}\left(6 b A \sigma^{2}+\left(A \sigma^{2}\right)^{2}\right) \\
\left.+H_{0,1, A}\left(4 b A \sigma^{2}+\left(A \sigma^{2}\right)^{2}\right)+H_{0,2, A}\left(8 b A \sigma^{2}+\left(A \sigma^{2}\right)^{2}\right)\right]=0 .
\end{gathered}
$$

Durch das Einsetzen der optimalen Menge $X_{0, *}$ in Gleichung (3.39) und Auflösen der Gleichung nach $F_{0,1}$ ergibt sich die Bewertungsgleichung für Forwards mit Verfallszeitpunkt in $t=1$ :

$$
\begin{aligned}
F_{0,1, *}\left(H_{0,1, *}, H_{0,2, *}\right)= & \frac{1}{12 b^{2}+8 b A \sigma^{2}+\left(A \sigma^{2}\right)^{2}}\left[P _ { 0 } \left(12 b^{2}+8 b A \sigma^{2}\right.\right. \\
& \left.+\left(A \sigma^{2}\right)^{2}\right)+\mu\left(12 b^{2}+2 b A \sigma^{2}\right) \\
& +H_{0,1, *}\left(12 b^{2} A \sigma^{2}+4 b\left(A \sigma^{2}\right)^{2}\right) \\
& \left.+H_{0,2, *}\left(12 b^{2} A \sigma^{2}+2 b\left(A \sigma^{2}\right)^{2}\right)\right] .
\end{aligned}
$$

Die Bewertungsgleichung für Forwards mit Verfallszeitpunkt in $t=2$ ergibt sich indem die optimale Menge $X_{0, *}$ in Gleichung (3.40) eingesetzt und nach $F_{0,2}$ aufgelöst wird:

$$
F_{0,2, *}\left(H_{0,1, *}, H_{0,2, *}\right)=\frac{4 H_{0,2, *} b A \sigma^{2}+2 H_{0,1, *} b A \sigma^{2}+P_{0} A \sigma^{2}+4 b \mu+2 P_{0} b}{2 b+A \sigma^{2}} .
$$

Die Gleichungen (3.41) und (3.42) zeigen, dass die Preise von Forwardkontrakte mit Verfallszeitpunkt in $t=1$ bzw. $t=2$ von allen Modellparametern abhängig sind. Beide Forwardpreise sind von der Nachfrage nach Kontrakten mit Verfallszeitpunkt in $t=1$ bzw. $t=2$ abhängig. Eine Nachfrage nach Longposition am Forwardmarkt erhöht beide Preise. Wie im Einperiodenmodell überträgt sich die Nachfrage dabei nicht eins zu eins sondern interagiert mit der Illiquidität und dem Risiko am Kassamarkt.

Die Bewertungsgleichungen (3.41) und (3.42) geben die Forwardpreise in Abhängigkeit der Nachfrage an. Diese allgemeinen Preise sind nicht beobachtbar. Ziel ist es empirisch überprüfbare Hypothesen abzuleiten und zu diesem Zweck müssen Preise verwendet werden, die beobachtbar sind. Es wird die spezielle Nachfrage von $H_{0,1, *}=H_{0,2, *}=1$ und $H_{0,1, *}=H_{0,2, *}=-1$ gewählt, da der Preis für die erste Einheit in der Realität dem Geldbzw. Briefkurs entspricht und beobachtbar ist. Anhand der Forwardbewertungsgleichun- 
gen (3.41) bzw. (3.42) kann der Midquote $\left(M I D_{0, t}\right)$ für Forwards mit Verfallszeitpunkt in $t=1$ bzw. $t=2$ somit explizit dargestellt werden durch:

$$
M I D_{0, t}=\frac{F_{0, t}(1,1)+F_{0, t}(-1,-1)}{2},
$$

mit $t \in\{1,2\}$. Speziell soll untersucht werden, wie die Forwardpreise auf einem illiquiden Markt von denen auf einem perfekt liquiden Markt abweichen. Der Referenzpreis für einen perfekt liquiden Markt ist der Preis aus dem Cost-of-Carry-Modell, welcher sich aus den Bewertungsgleichungen (3.41) und (3.42) für den Fall $b=0$ ergibt. Ohne die Berücksichtigung von Zinseffekten ist der Cost-of-Carry-Preis $(C o C)$ folglich sowohl für Forwards mit Verfallszeitpunkt in $t=1$ als auch in $t=2$ gegeben durch $P_{0}{ }^{8}$ Die Abweichung des Midquotes eines illiquiden Forwards mit Verfallszeitpunkt in $t=1$ vom Cost-of-Carry-Preis ergibt sich damit als:

$$
M I D_{0,1}-C o C=\frac{2 \mu b}{2 b+A \sigma^{2}}
$$

Die Abweichung des Forwards mit Verfallszeitpunkt in $t=1$ vom Cost-of-Carry-Preis wird getrieben durch die erwartete Rendite, die Illiquidität sowie das Risiko am Kassamarkt. Dabei nimmt die erwartete Rendite eine entscheidende Rolle ein. Für den Fall einer positiven erwarteten Rendite liegt der Preis des illiquiden Forwards über dem Costof-Carry-Preis. Dabei ist die Abweichung umso größer, je höher die erwartete Rendite ist. Eine hohe erwartete Rendite lässt eine Longposition am Kassamarkt attraktiver werden. Besteht eine Nachfrage nach Longpositionen am Forwardmarkt, hält der Market Maker eine Netto-Shortposition. Folglich erhöht er den Forwardpreis mit steigender erwarteter Rendite, damit weniger Investoren Longpositionen in Forwards nachfragen. Für den Fall einer negativen erwarteten Rendite liegt der Preis des illiquiden Forwards unter dem Cost-of-Carry-Preis. Eine höhere Illiquidität am Kassamarkt bewirkt, dass der Absolutbetrag der Abweichung vom Cost-of-Carry-Preis für $\mu \neq 0$ größer wird. Mit der Illiquidität am Kassamarkt steigen ebenfalls die Transaktionskosten des Market Makers. Folglich setzt er die Preise so, dass eine Investition in Forwards weniger attraktiv wird und er damit geringere Mengen am Kassamarkt zur Absicherung handeln muss. Ein steigendes Risiko am Kassamarkt verringert den Absolutbetrag der Abweichung vom Cost-of-Carry-Preis für $\mu \neq 0$. Dieser Effekt ist dadurch zu erklären, dass die Abwei-

\footnotetext{
${ }^{8}$ Die Berücksichtigung von Zinseffekten erfolgt in der anschießenden empirischen Analyse.
} 
chung vom Cost-of-Carry-Preis durch die spekulative Komponente des Market Makers getrieben wird und der Market Maker mit steigendem Risiko weniger spekuliert. Zusammenfassend lassen sich zwei empirisch testbare Hypothesen zu den Preiseffekten am Forwardmarkt formulieren:

HP1: Je höher die Illiquidität am Kassamarkt, desto höher der Absolutbetrag der Abweichung vom Cost-of-Carry-Preis am Forwardmarkt.

HP2: Je höher das Risiko am Kassamarkt, desto höher der Absolutbetrag der Abweichung vom Cost-of-Carry-Preis am Forwardmarkt.

Die Differenz zwischen dem Midquote eines illiquiden Forwards mit Verfallszeitpunkt in $t=2$ und dem Cost-of-Carry-Preis entspricht:

$$
M I D_{0,2}-C o C=\frac{4 \mu b}{2 b+A \sigma^{2}} .
$$

Die Auswirkungen der erwarteten Rendite, der Illiquidität und dem Risiko am Kassamarkt auf den Absolutbetrag der Abweichung vom Cost-of-Carry-Preis sind für einen Forward mit Verfallszeitpunkt in $t=2$ und einen Forward mit Verfallszeitpunkt in $t=1$ identisch. Um die Unterschiede in den Abweichungen vom Cost-of-Carry-Preis von Forwards mit Verfallszeitpunkt in $t=2$ und Forwards mit Verfallszeitpunkt in $t=1 \mathrm{zu}$ analysieren, wird die Differenz der Gleichungen (3.45) und (3.44) gebildet:

$$
M I D_{0,2}-M I D_{0,1}=\frac{2 \mu b}{2 b+A \sigma^{2}}
$$

Gleichung (3.46) zeigt, dass gegeben $\mu \neq 0$ der Absolutbetrag der Abweichung vom Cost-of-Carry-Preis für Forwards mit Verfallszeitpunkt in $t=2$ größer ist, als die für Forwards mit Verfallszeitpunkt in $t=1$. Damit lässt sich folgende dritte empirisch testbare Preishypothese ableiten:

HP3: Der Absolutbetrag der Abweichung vom Cost-of-Carry-Preis am Forwardmarkt ist größer für Forwardkontrakte mit längerer Restlaufzeit als für Forwardkontrakte mit kürzerer Restlaufzeit.

\subsubsection{Liquiditätseffekte}

Zur Untersuchung der Liquiditätseffekte von Forwardkontrakten mit unterschiedlichen Laufzeiten können anhand der Bewertungsgleichungen (3.41) und (3.42) die entsprechen- 
den Illiquiditätsmaße bestimmt werden. Die Illiquiditätsmaße für Forwardkontrakte mit Verfallszeitpunkt in $t=1$ und $t=2$ ergeben sich als:

$$
I L M_{0,1}=\frac{4 b A \sigma^{2}\left(3 b+A \sigma^{2}\right)}{12 b^{2}+8 b A \sigma^{2}+\left(A \sigma^{2}\right)^{2}}
$$

und

$$
I L M_{0,2}=\frac{4 b A \sigma^{2}}{2 b+A \sigma^{2}}
$$

Die Illiquiditätsmaße zeigen, dass sowohl die Illiquidität des Forwards mit Verfallszeitpunkt in $t=1$ als auch in $t=2$ gegen null geht, sofern der Kassamarkt perfekt liquide oder risikolos ist. Für beide Kontrakte wirkt sich eine steigende Illiquidität des Kassamarktes oder ein steigendes Risiko am Kassamarkt negativ auf die Forwardliquidität aus. Die Illiquiditätsübertragung auf Forwards mit unterschiedlicher Laufzeit unterscheidet sich damit nicht wesentlich von der Übertragung im Einperiodenmodell.

Eine zentrale Frage die im Rahmen des Mehrperiodenmodells beantwortet werden soll ist, ob Forwards mit Verfallszeitpunkt in $t=2$ unabhängig von den Modellparametern eine höhere Illiquidität als Forwards mit Verfallszeitpunkt in $t=1$ aufweisen. Um diese Frage zu beantworten, wird die Differenz der Illiquiditätsmaße $I L M_{0,2}$ und $I L M_{0,1}$ berechnet:

$$
I L M_{0,2}-I L M_{0,1}=\frac{12 b^{2} A \sigma^{2}}{12 b^{2}+8 b A \sigma^{2}+\left(A \sigma^{2}\right)^{2}} .
$$

Die Differenz hängt von der Illiquidität und Volatilität am Kassamarkt sowie der Risikoaversion des Market Makers ab. Sämtliche dieser Parameter sind strikt größer null. Damit ist auch der Bruch (3.49) größer null, was bedeutet, dass Forwards mit Verfallszeitpunkt in $t=2 \mathrm{im}$ Mehrperiodenmodell unabhängig von der Parameterwahl illiquider sind als Forwards mit Verfallszeitpunkt in $t=1$. Als vierte empirisch testbare Hypothese zu den Liquiditätseffekten wird damit die folgende Hypothese formuliert:

HL4: Forwardkontrakte mit längerer Restlaufzeit sind illiquider als Forwardkontrakte kürzerer Restlaufzeit. 


\section{Empirische Analyse der Preis- und Liquiditätseffekte}

Gegenstand dieses Kapitels ist die empirische Analyse der in Kapitel 3 abgeleiteten Preisund Liquiditätshypothesen. Im ersten Teil werden zur Überprüfung der Hypothesen geeignete Aktien- und Futuresmärkte vorgestellt und die Besonderheiten dieser Märkte erläutert. Der zweite und dritte Teil des Kapitels hat die Untersuchung der Preis- und Liquiditätseffekte zum Thema.

\subsection{Untersuchte Aktien- und Futuresmärkte}

Zunächst müssen geeignete Aktien- und Futuresmärkte ausgewählt werden, um die Preisund Liquiditätseffekte empirisch untersuchen zu können. Der einfachste Futureskontrakt ist ein sogenannter SSF. Ein SSF ist ein relativ neues Derivat, das in der Funktionsweise mit den etablierten Indexfutures vergleichbar ist. Der wesentliche Unterschied besteht in dem zugrundeliegenden Basisinstrument, welches im Fall eines SSF eine Einzelaktie ist und nicht wie im Fall von Indexfutures ein Aktienindex. Wird ein SSF-Kontrakt zwischen zwei Parteien abgeschlossen, verpflichtet sich eine Partei die dem Kontrakt zugrunde liegende Aktie zu einem zukünftigen Zeitpunkt und festgelegten Preis zu kaufen, die andere Partei nimmt die Gegenseite dieser Position ein.

Eine Einsatzmöglichkeit für SSF besteht folglich in der Absicherung gegenüber Preisveränderungen von Einzelaktien. Des Weiteren wird das Eingehen von Shortpositionen erleichtert, da bei einer SSF-Shortposition im Gegensatz zu einem Leerverkauf keine Gebühren durch das Leihen einer Aktie anfallen. Darüber hinaus wird keine Partei benötigt, die bereit ist, die entsprechende Aktie zu verleihen. Danielsen et al. (2009) finden empirische Evidenz für den US-Markt, dass SSF-Shortpositionen ein günstiges Substitut gegenüber Leerverkäufen darstellen. Ein weiterer Vorteil von SSF besteht in ihrer Hebelwirkung, d. h. mit SSF ist es Investoren möglich, große Positionen im Verhältnis zum eingesetzten Kapital aufzubauen. Häufigen Einsatz finden SSF beim sogenannten Dividendenstripping, einer Strategie, bei der die Zahlung einer Dividende umgangen wird. SSF eignen sich für eine solche Strategie, da die Zahlung einer Dividende nur an den 
Halter einer Aktie und nicht an den Halter des entsprechenden SSF erfolgt. Die Vermeidung einer Dividendenzahlung kann erfolgen, indem der Investor die Aktie vor dem Ex-Tag verkauft und gleichzeitig eine Longposition in einem SSF auf die entsprechende Aktie eingeht. Auf diese Weise bleibt das Exposure seines Portfolios unverändert, ohne dass eine Dividende an den Investor gezahlt wird. Es werden zwei Annahmen getroffen, um zu plausibilisieren wie ein Investor von dieser Strategie profitieren könnte: Erstens wird von Risikoprämien abstrahiert und zweitens wird unterstellt, dass beim Verkauf der Aktie kein zu versteuernder Gewinn realisiert wird. Am Ex-Tag wird die Futuresposition glattgestellt und die Aktie zurückgekauft. Der erwartete Gewinn aus der SSF-Position ist gleich null, da der Kursabschlag im Basiswertpapier vom SSF antizipiert wird. Der Gewinn aus der Aktienposition entspricht der Höhe des Kursabschlags. Ist der Kursabschlag höher als die nach Steuern verbleibende Dividende, können Investoren mit dieser Strategie ihre Steuerlast senken. Hinweise auf eine entsprechende Strategie finden Bialkowski \& Jakubowski (2012), indem sie zeigen, dass das Handelsvolumen der an der European Exchange (Eurex) gehandelten SSF um den Ex-Tag ansteigt.

Aus drei Gründen sind SSF das ideale Instrument, um die aus dem Modell abgeleiteten Preis- und Liquiditätshypothesen zu testen: Erstens kann das Hedging eines SSF mit einem einzigen Instrument, dem zugrundeliegenden Basiswertpapier, erfolgen. Dies spiegelt die Struktur des Modells wider. Zweitens lassen sich sowohl der Mittelpunkt der Geld-Brief-Spanne als auch die Liquidität eines SSF und dessen Basiswertpapiers einfach messen. Drittens sind SSF auf verschiedene Aktien verfügbar, was es ermöglicht, einen Paneldatensatz zu konstruieren, um so sowohl die Zeitreihen- als auch die Querschnittseigenschaften zu analysieren. Indexfutures sind insbesondere aufgrund der ersten zwei Argumente nicht optimal für die Überprüfung der aus dem Modell abgeleiteten Hypothesen geeignet. Sie können nicht über ein einziges Instrument abgesichert werden, sondern müssen über ein Aktienportfolio gehedgt werden. Für dieses Hedgeportfolio existiert darüber hinaus weder eine klar definierte Geld-Brief-Spanne noch ein einfaches Liquiditätsmaß.

Zur Überprüfung der Hypothesen müssen SSF gewählt werden, die an einer Börse gehandelt werden deren Marktstruktur die des Modells reflektiert. Darüber hinaus müssen der Mittelpunkt der Geld-Brief-Spanne und die Liquidität auf dem Aktien- und Futuresmarkt auf eine konsistente Weise gemessen werden können. Diese Anforderungen werden von den Unternehmen im DAX30 und den entsprechenden SSF erfüllt. Die 
DAX30 Aktien sind die wichtigsten deutschen Aktien und machen $83 \%$ der gesamten Marktkapitalisierung des deutschen Aktienmarktes aus. ${ }^{1}$

Die SSF auf deutsche Aktien werden an der Eurex über ein zentrales Limit-Orderbuch gehandelt. Sofern sich zwei Parteien einigen, können SSF außerdem Over-The-Counter (OTC) gehandelt werden. Zur Sicherung der Liquidität und damit auch der Preisqualität existieren für die DAX30 SSF sogenannte designierte Market Maker, die zusätzliche Liquidität durch das gleichzeitige Einstellen von Geld- und Briefkursen zur Verfügung stellen. Die Marktstruktur an der Eurex für SSF ist damit nicht rein ordergetrieben, sondern eine Kombination aus Order- und Dealermarkt. Auch für die Marktstruktur des Modells gilt, dass Investoren untereinander handeln können und lediglich der Angebotsbzw. Nachfrageüberschuss in Höhe von $H_{*}$ durch den repräsentativen Market Maker bedient wird. Folglich ist die Marktstruktur der Eurex ideal, um die Modellhypothesen zu überprüfen. Die empirische Analyse beschränkt sich auf die DAX30 SSF, da die designierten Market Maker nur für diese SSF die Liquidität gewährleisten (Eurex (2015)).

Eingeführt wurden SSF an der Eurex im Oktober 2005. Seitdem ist die Zahl der angebotenen SSF sowie das Handelsvolumen stetig gestiegen. Inzwischen werden mehr als 900 SSF auf Aktien aus 20 verschiedenen Ländern angeboten. Die Eurex ist einer der wichtigsten Märkte für SSF weltweit und besaß im Jahr 2013 einen Marktanteil von 19\%, was einem Volumen von 946,3 Millionen Kontrakten entsprach. Institutionelle Investoren bilden den Großteil der am Markt für SSF aktiven Investoren (Eurex (2014)).

Das Modell macht keine Aussage zur Marktstruktur am Kassamarkt. Folglich ist es nicht entscheidend, dass die Marktstrukturen am SSF- und Aktienmarkt vergleichbar sind, vereinfacht jedoch die empirische Analyse. Der Handel an der deutschen Börse erfolgt über Xetra, die vollelektronische Handelspattform der deutschen Börse AG für Aktien. Das Marktmodell für den Aktienhandel ist wie das der Eurex für SSF ordergetrieben. Die Liquidität wird folglich durch die Investoren selbst und deren Einstellen von Limit-Orders bereitgestellt. Für bedeutende Aktien wird die Liquidität und Preisqualität darüber hinaus durch sogenannte designierte Sponsoren sichergestellt. Fällt die Liquidität einer solchen Aktie unter einen von der Börse festgelegten Wert, sind die Banken und Wertpapierhäuser, welche als designierte Sponsoren agieren, verpflichtet Geld- und Briefkurse zu stellen (Xetra (2015)). Der Handelsmechanismus für die DAX30 Aktien und SSF ist folglich vergleichbar.

${ }^{1}$ Dieser Wert ist dem Factbook der Deutschen Börse (2009) entnommen und bezieht sich auf das Jahresende 2009, den Beginn des Untersuchungszeitraums. 
Die Geld-Brief-Spannen stehen sowohl für Aktien als auch für SSF zur Verfügung. Darüber hinaus wird von der deutschen Börse für beide Märkte das gleiche Liquiditätsmaß berechnet. Dadurch kann sichergestellt werden, dass die Liquidität auf beiden Märkten auf identische Weise gemessen wird.

\subsection{Preiseffekte}

Die Analyse der Preiseffekte gliedert sich in drei Teile. Der erste Teil stellt die Datenbasis vor, definiert die verwendeten Variablen und erläutert die erwarteten Effekte. Deskriptive Statistiken der zentralen Variablen werden im zweiten Teil des Abschnittes dargestellt. Die genaue Untersuchung der Preiseffekte am SSF-Markt inklusive verschiedener Robustheitstests bildet den letzten Teil dieses Abschnitts.

\subsubsection{Variablen und erwartete Effekte}

Auf friktionslosen Märkten sollten Futures zum Cost-of-Carry-Preis gehandelt werden. Daher dient dieser als Benchmark für die empirische Analyse. Für die Überprüfung der Preishypothesen müssen folglich die Abweichungen der SSF-Midquotes von den Costof-Carry-Preisen bestimmt werden. Die Berechnung der Cost-of-Carry-Preise erfolgt auf Basis der Aktienmidquotes. Die Midquotes der SSF und Aktien ergeben sich als Durchschnitt aus den entsprechenden Geld- und Briefkursen. Damit die Abweichung adäquat gemessen werden kann, müssen sowohl die Geld- und Briefkurse der SSF als auch die der Aktien zum gleichen Zeitpunkt gemessen werden. Aus diesem Grund ist es nicht möglich Schlusskurse für die empirische Analyse zu verwenden, da der Handel an der Eurex um 17.45 Uhr endet, der Handel auf Xetra jedoch bereits um 17.30 Uhr. Um 16.00 Uhr befinden sich beide Märkte im fortlaufenden Handel. Daher werden die zu diesem Zeitpunkt gültigen Geld- und Briefkurse der SSF und Aktien zur Bestimmung der Midquotes verwendet. ${ }^{2}$ Die SSF-Daten sind für den Zeitraum vom Januar 2010 bis zum April 2013 über die Thompson Reuters Tick History verfügbar. Die Daten der Aktien werden für den gleichen Zeitraum direkt über die deutsche Börse bezogen. Berechnet

\footnotetext{
${ }^{2}$ Stehen zu diesem Zeitpunkt mehrere Geld- und/oder Briefkurse zur Verfügung, werden die Kurse mit der kleinsten Geld-Brief-Spanne verwendet.
} 
werden die Cost-of-Carry-Preise anhand der folgenden Formel:

$$
\operatorname{CoC}_{t}(T)=M I D_{A, t}\left(1+r_{t, T}(T-t)\right)-\operatorname{Div}\left(1+r_{t, t_{D}, T}\left(T-t_{D}\right)\right),
$$

mit $C o C_{t}(T)$ als Cost-of-Carry-Preis eines SSF im Zeitpunkt $t$ mit Verfallszeitpunkt $T$, $M I D_{A, t}$ als Aktienmidquote im Zeitpunkt $t, r_{t, T}$ als Zins im Zeitpunkt $t$ für eine Laufzeit der Länge $(T-t)$, Div als Dividendenzahlung in Euro, $t_{D}$ als Ex-Tag ${ }^{3}$ und $r_{t, t_{D}, T}$ als Terminzins im Zeitpunkt $t$ für eine Laufzeit der Länge $\left(T-t_{D}\right)$.

Für die Berechnung der Cost-of-Carry-Preise werden die Zinssätze auf dem Interbankenmarkt für Termingelder in Euro verwendet. Als Ein-Tages-Zinssatz wird der Euro Over Night Index Average (EONIA) und als Drei-Monats-Zinssatz die Euro Inter Bank Offered Rate (EURIBOR) verwendet. Beide Zinssätze stehen über Datastream zur Verfügung. Der Untersuchungszeitraum liegt in der beginnenden Niedrigzinsphase mit einem mittleren Drei-Monats-EURIBOR (EONIA) von 0,85\% p. a. (0,47\% p. a.). Die Ein-Tages- und Drei-Monats-Zinsen sind zu jedem Zeitpunkt positiv. Zinsen für eine Laufzeit zwischen einem Tag und drei Monaten werden durch lineare Interpolation bestimmt. Zinsen für eine Laufzeit größer als drei Monate werden durch Extrapolation bestimmt. Auch durch dieses Vorgehen kommt es zu keinem Zeitpunkt zu negativen Zinsen. Der maximale Zinssatz im Untersuchungszeitraum liegt bei 3,15\% p. a. Ausreißer in den Cost-of-Carry-Preisen sind folglich nicht auf die verwendeten Zinssätze zurückzuführen. Aufgrund der unterschiedlichen Preisniveaus werden in der empirischen Analyse relativen Abweichungen von den Cost-of-Carry-Preisen $\left(A B W_{C o C, t}(T)\right)$ verwendet

$$
A B W_{C o C, t}(T)=\frac{M I D_{F, t}(T)-C o C_{t}(T)}{C o C_{t}(T)},
$$

mit $M I D_{F, t}$ als Midquote eines SSF im Zeitpunkt $t$ mit Verfallszeitpunkt $T$.

Durch das in Abschnitt 3.2 entwickelte Modell konnten folgende fünf Faktoren identifiziert werden, welche die Abweichungen der SSF-Midquotes von den Cost-of-CarryPreisen beeinflussen: Die erwartete Rendite, Illiquidität und Volatilität des Basiswertpapiers sowie die Restlaufzeit der SSF-Kontrakte und die Risikoaversion des Market Makers.

Eine Schwierigkeit, die Modellvorhersagen empirisch zu überprüfen, besteht darin, dass

${ }^{3}$ Tatsächlich können Zinseinkünfte erst ab der Auszahlung der Dividende generiert werden. Der Zeitraum zwischen Ex-Tag und Auszahlung der Dividende ist in der Realität gering. In dieser Arbeit wird der Ex-Tag verwendet. 
das Vorzeichen der Abweichung von der erwarteten Rendite im Beobachtungszeitraum abhängt. Für den Fall einer positiven (negativen) erwarteten Rendite liegt der SSFMidquote über (unter) dem Cost-of-Carry-Preis. Die Auswirkungen der übrigen Faktoren auf den SSF-Midquote sind ebenfalls abhängig von der erwarteten Rendite. Bei positiver erwarteter Rendite würde zum Beispiel eine steigende Illiquidität des Basiswertpapiers einen zusätzlichen Anstieg des Midquotes bewirken. Ist die erwartete Rendite dagegen negativ würde eine steigende Illiquidität den Midquote sinken lassen. Um dieser Problematik Rechnung zu tragen, wird in der empirischen Analyse der Absolutbetrag der relativen Abweichungen von den Cost-of-Carry-Preisen (abs_ABW $\left.W_{C o C}\right)$ als abhängige Variable betrachtet. Die erwartete Rendite wird nicht als erklärende Variable in die empirische Analyse aufgenommen, da die erwartete Rendite insbesondere über kurze Zeiträume schwer zu schätzen ist (Merton (1980)).

Im Modell tritt die Nachfrage $H_{*}$ bei der Bestimmung des Midquotes nicht auf. Grundsätzlich könnte diese jedoch einen Einfluss auf die Höhe der SSF-Midquotes haben. Gibt es eine Nachfrage nach SSF-Longpositionen, könnte der Market Maker diese Position unattraktiver werden lassen, indem er eine Geld-Brief-Spanne stellt, so dass der Midquote des SSF über dem Cost-of-Carry-Preis liegt. Eine Geld-Brief-Spanne, deren Midquote kleiner als der Cost-of-Carry-Preis ist, würde der Market Maker dagegen bei einer Nachfrage nach SSF-Shortpositionen stellen. Ein weiteres Argument für die Analyse des Absolutbetrags der Abweichung ist, dass anhand der verfügbaren Daten nicht festgestellt werden kann, ob eine Nachfrage nach Long- oder Shortpositionen vorliegt.

Ziel der empirischen Analyse ist es herauszufinden, welche Faktoren den Absolutbetrag der relativen Abweichungen von den Cost-of-Carry-Preisen treiben. Die erste aus dem Modell in Abschnitt 3.2 abgeleitete Hypothese ist, dass der Absolutbetrag der Preisabweichungen am SSF-Markt durch eine steigende Illiquidität am Aktienmarkt ebenfalls steigen sollte. Die Illiquidität am Aktienmarkt wird durch die relative Geld-Brief-Spanne am Aktienmarkt $\left(G B S_{A}\right)$ um 16.00 Uhr gemessen. Berechnet wird die relative GeldBrief-Spanne als Quotient aus der Geld-Brief-Spanne und dem Midquote. Folglich wird $G B S_{A}$ als erste erklärende Variable in die empirische Analyse aufgenommen und ein positiver Zusammenhang zum Absolutbetrag der Preisabweichungen erwartet.

Eine hohe Volatilität und Risikoaversion sollten nach dem Modell in Abschnitt 3.2 dagegen den Absolutbetrag der Preisabweichungen am SSF-Markt verringern. Modelltheoretisch wird die Abweichung vom Cost-of-Carry-Preis durch die spekulative Komponente des Market Makers getrieben. Mit steigendem Risiko wird die spekulative Komponente 
kleiner und damit auch die Abweichung vom Cost-of-Carry-Preis. Die Literatur zu Indexfutures lässt dagegen die entgegengesetzte Wirkung erwarten. Verschiedene Studien zeigen, dass ein höheres Risiko zu größeren Fehlbewertungen am Futuresmarkt führt (vgl. z. B. Yadav \& Pope (1994)). Für die empirische Analyse der Auswirkungen des Risikos werden die Faktoren Risikoaversion des Market Makers und Volatilität des Basiswertpapiers nicht separat gemessen, da die Risikoaversion des Market Maker schwer $\mathrm{zu}$ messen ist und verschiedene Studien gezeigt haben, dass diese hoch ist, wenn ebenfalls die Volatilität hoch ist (vgl. z. B. Bekaert et al. (2013)). Als Risikomaß wird die annualisierte Standardabweichung der täglichen Aktienrenditen (Volatilität) über ein rollierendes Fenster von 30 Handelstagen geschätzt und als zweite erklärende Variable in die empirische Analyse aufgenommen. Die benötigten Daten stehen über Datastream zur Verfügung.

Die letzte aus dem Modell abgeleitete Hypothese ist, dass der Absolutbetrag der Preisabweichungen für langfristige SSF größer ist als der für kurzfristige SSF. Für die empirische Analyse wird daher für jeden Tag der Absolutbetrag der Preisabweichung für den SSF mit der kürzesten Restlaufzeit sowie für den SSF mit der zweitkürzesten Restlaufzeit berechnet. Zur Untersuchung der Laufzeiteffekte wird eine Dummy Variable verwendet, die den Wert 0 für die SSF mit der kürzesten Restlaufzeit und 1 für die SSF mit der zweitkürzesten Restlaufzeit annimmt. Die Dummy Variable (Laufzeit) wird als dritte erklärende Variable in der empirischen Analyse verwendet.

\subsubsection{Deskriptive Statistik}

In diesem Abschnitt werden zunächst deskriptive Statistiken der Abweichungen von den Cost-of-Carry-Preisen untersucht. Es wird dabei zwischen den Preisabweichungen von SSF mit der kürzesten und zweitkürzesten Restlaufzeit unterschieden. Kurz vor dem Verfallsdatum kommt es durch das Rollen der SSF zu einem Anstieg des Handelsvolumens. Um daraus resultierende Verzerrungen zu vermeiden, werden SSF im Verfallsmonat von der empirischen Analyse ausgeschlossen (Bialkowski \& Jakubowski (2012)). Darüber hinaus werden in der Analyse die Geld-Brief-Spannen der Aktien und SSF mit Werten kleiner gleich null oder größer als 1000 Basis Punkte (BP) nicht berücksichtigt. Dieser Filter dient dazu ökonomisch nicht plausible Werte von der Analyse auszuschließen. Durch dieses Vorgehen werden weniger als 1\% der Beobachtungen gelöscht. 
Abbildung 4.1: Relative Abweichungen der SSF-Preise von den Cost-of-Carry-Preisen

Panel A: kurzfristige SSF

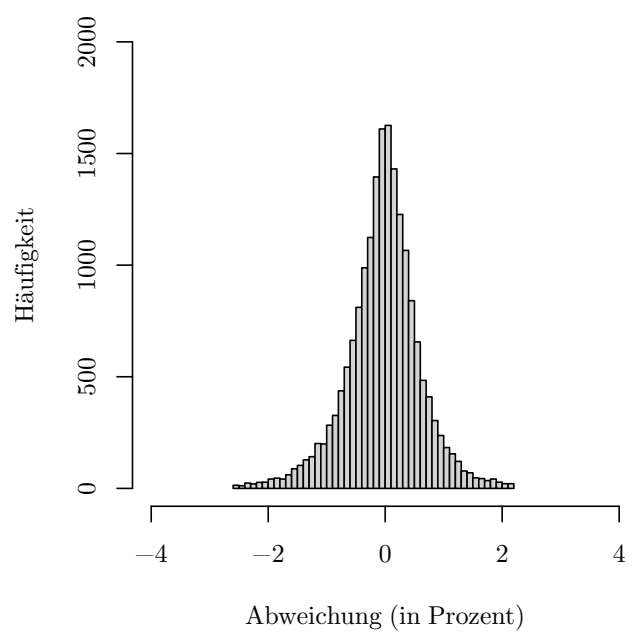

Panel B: langfristige SSF

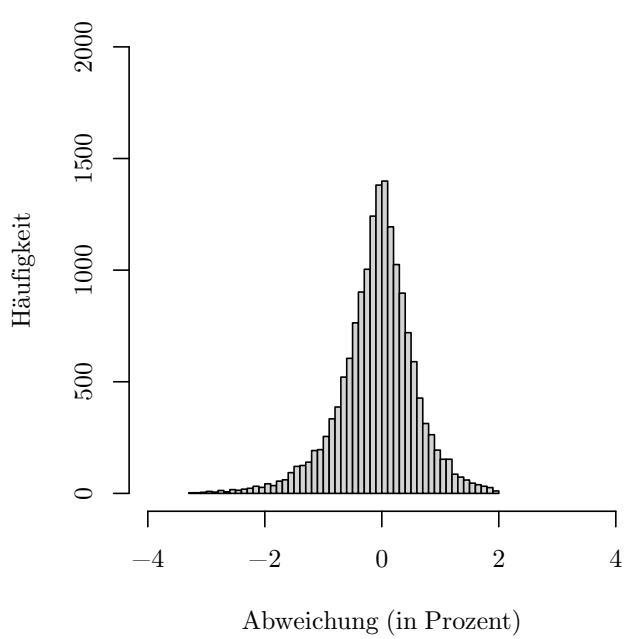

Diese Abbildung zeigt die relativen Abweichungen der kurz- und langfristigen SSF-Midquotes von den Cost-of-CarryPreisen. Der Beobachtungszeitraum ist vom 01.01.2010 bis zum 01.04.2013.

Zunächst werden die relativen Abweichungen allgemein und im Anschluss daran der Absolutbetrag der relativen Abweichungen untersucht. Dazu werden die relativen Differenzen der empirischen Midquotes und Cost-of-Carry-Preise für kurz- und langfristige SSF bestimmt und in Abbildung 4.1 in Form zweier Histogramme dargestellt. Panel A zeigt die Preisabweichungen der kurzfristigen SSF und Panel B die der langfristigen SSF. Anhand des Histogramms in Panel A wird deutlich, dass es keine eindeutige Überoder Unterbewertung in Bezug auf den Cost-of-Carry-Preis von kurzfristigen SSF gibt. Die empirischen Midquotes liegen zu ca. 51\% (49\%) unter (über) dem Cost-of-CarryPreis. Insgesamt stehen 20.039 Beobachtungen zur Verfügung. Der Mittelwert und die Standardabweichung der relativen Abweichungen liegen bei -0,03\% und 0,63\%. Das Minimum (Maximum) liegt bei $-2,59 \%$ (2,19\%). Auch für langfristige SSF zeigt sich in Panel B der Abbildung 4.1 keine extreme Unter- bzw. Überbewertung der SSF. Von insgesamt 17.497 Beobachtungen liegen 53\% (47\%) der langfristigen SSF-Midquotes unter (über) dem Cost-of-Carry-Preis. Mit einem Mittelwert von -0,08\%, einer Standardabweichung von 0,65\% und einem Minimum (Maximum) von -3,28\% (1,94\%) ist die Unterbewertung der langfristigen SSF etwas ausgeprägter als die der kurzfristigen SSF. 
Tabelle 4.1: Absolutbetrag der relativen Abweichungen der SSF-Preise von den Cost-of-Carry-Preisen

\begin{tabular}{|c|c|c|c|c|c|c|}
\hline & \multicolumn{6}{|c|}{ Absolutbetrag der Preisabweichungen (in Prozent) } \\
\hline & \multicolumn{3}{|c|}{ kurzfristige SSF } & \multicolumn{3}{|c|}{ langfristige SSF } \\
\hline & Mittelwert & Median & $\mathrm{N}$ & Mittelwert & Median & $\mathrm{N}$ \\
\hline Merck & 0,3167 & 0,2369 & 400 & 0,2936 & 0,2145 & 248 \\
\hline Henkel & 0,3232 & 0,3384 & 3 & 0,3250 & 0,3678 & 3 \\
\hline Fresenius Medical Care & 0,3481 & 0,2475 & 847 & 0,3497 & 0,2486 & 698 \\
\hline Beiersdorf & 0,3688 & 0,2563 & 506 & 0,3818 & 0,2940 & 371 \\
\hline SAP & 0,3716 & 0,2921 & 653 & 0,3734 & 0,2930 & 628 \\
\hline Linde & 0,3768 & 0,2566 & 850 & 0,3595 & 0,2508 & 701 \\
\hline $\mathrm{K}+\mathrm{S}$ & 0,3816 & 0,3217 & 299 & 0,3618 & 0,3046 & 203 \\
\hline Deutsche Telekom & 0,3820 & 0,2962 & 850 & 0,4776 & 0,3180 & 791 \\
\hline Deutsche Post & 0,3908 & 0,3002 & 850 & 0,3895 & 0,2934 & 703 \\
\hline RWE & 0,4161 & 0,3063 & 674 & 0,4468 & 0,3240 & 533 \\
\hline Adidas & 0,4231 & 0,3231 & 849 & 0,4327 & 0,3339 & 703 \\
\hline MAN & 0,4234 & 0,2851 & 380 & 0,4933 & 0,3478 & 332 \\
\hline Siemens & 0,4248 & 0,3297 & 850 & 0,4348 & 0,3269 & 821 \\
\hline Münchener Rück & 0,4300 & 0,3057 & 544 & 0,4269 & 0,3139 & 512 \\
\hline Metro & 0,4314 & 0,3179 & 697 & 0,4234 & 0,3308 & 588 \\
\hline Bayer & 0,4356 & 0,3104 & 850 & 0,4406 & 0,3220 & 793 \\
\hline Allianz & 0,4553 & 0,3377 & 850 & 0,4651 & 0,3444 & 850 \\
\hline E.ON & 0,4643 & 0,3360 & 835 & 0,4758 & 0,3644 & 830 \\
\hline Lufthansa & 0,4747 & 0,3753 & 848 & 0,5022 & 0,3903 & 703 \\
\hline BASF & 0,4868 & 0,3622 & 836 & 0,4800 & 0,3519 & 778 \\
\hline ThyssenKrupp & 0,5007 & 0,3915 & 832 & 0,5279 & 0,4216 & 730 \\
\hline BMW & 0,5230 & 0,3759 & 850 & 0,5580 & 0,3813 & 725 \\
\hline Daimler & 0,5294 & 0,3832 & 850 & 0,5478 & 0,4118 & 789 \\
\hline Volkswagen Vz. & 0,5336 & 0,3953 & 416 & 0,5525 & 0,4098 & 298 \\
\hline Deutsche Börse & 0,5372 & 0,3765 & 398 & 0,4100 & 0,3136 & 267 \\
\hline HeidelbergCement & 0,5527 & 0,4134 & 734 & 0,5614 & 0,4312 & 623 \\
\hline Salzgitter & 0,5609 & 0,6231 & 19 & 0,5239 & 0,5239 & 2 \\
\hline Deutsche Bank & 0,5881 & 0,4626 & 850 & 0,5907 & 0,4724 & 809 \\
\hline Infinion & 0,5996 & 0,4558 & 850 & 0,6160 & 0,4494 & 849 \\
\hline Commerzbank & 0,6057 & 0,4408 & 404 & 0,7138 & 0,6017 & 357 \\
\hline Fresenius & 0,6881 & 0,5941 & 365 & 0,6919 & 0,6630 & 259 \\
\hline
\end{tabular}

Diese Tabelle zeigt den Mittelwert, den Median sowie die Anzahl der täglichen Beobachtungen (N) des Absolutbetrags der relativen Abweichungen der kurz- und langfristigen SSF-Midquotes von den Cost-of-Carry-Preisen $\left(a b s \_A B W_{C o C}\right)$. Der Beobachtungszeitraum ist vom 01.01.2010 bis zum 01.04.2013.

Gegenstand der empirischen Analyse ist es zu untersuchen, ob der Absolutbetrag der Preisabweichungen in einem Zusammenhang zu der Friktion eines illiquiden Aktienmarkes steht. In Tabelle 4.1 werden der Mittelwert und Median sowie die Anzahl der Beobachtungen der Preisabweichungen der kurz- und langfristigen SSF auf Unternehmensebene dargestellt. Die geringste mittlere Abweichung weist der SSF auf die Merck 
Tabelle 4.2: Illiquidität und Volatilität der Aktien

\begin{tabular}{|c|c|c|c|c|c|c|}
\hline & \multicolumn{3}{|c|}{$G B S_{A}$ (in Prozent) } & \multicolumn{3}{|c|}{ Volatilität (in Prozent) } \\
\hline & Mittelwert & Median & $\mathrm{N}$ & Mittelwert & Median & $\mathrm{N}$ \\
\hline Siemens & 0,0386 & 0,0442 & 834 & 23,45 & 22,35 & 869 \\
\hline SAP & 0,0471 & 0,0540 & 834 & 19,91 & 18,49 & 869 \\
\hline BASF & 0,0476 & 0,0553 & 814 & 26,75 & 25,26 & 869 \\
\hline Allianz & 0,0483 & 0,0572 & 834 & 26,79 & 22,74 & 869 \\
\hline RWE & 0,0502 & 0,0582 & 814 & 25,96 & 22,58 & 869 \\
\hline Bayer & 0,0532 & 0,0618 & 834 & 25,53 & 23,78 & 869 \\
\hline Daimler & 0,0539 & 0,0606 & 834 & 31,07 & 29,42 & 869 \\
\hline Deutsche Bank & 0,0550 & 0,0642 & 814 & 36,67 & 33,36 & 869 \\
\hline E.ON & 0,0571 & 0,0665 & 814 & 26,44 & 23,72 & 869 \\
\hline BMW & 0,0573 & 0,0676 & 834 & 31,08 & 29,12 & 869 \\
\hline Fresenius Medical Care & 0,0596 & 0,0729 & 814 & 18,07 & 17,04 & 869 \\
\hline Henkel & 0,0651 & 0,0764 & 834 & 21,21 & 20,16 & 869 \\
\hline Adidas & 0,0656 & 0,0813 & 834 & 25,41 & 23,80 & 869 \\
\hline Merck & 0,0685 & 0,0836 & 834 & 22,24 & 20,25 & 869 \\
\hline Beiersdorf & 0,0696 & 0,0851 & 834 & 18,28 & 17,44 & 869 \\
\hline Deutsche Börse & 0,0709 & 0,1132 & 814 & 27,66 & 25,22 & 869 \\
\hline ThyssenKrupp & 0,0714 & 0,0839 & 834 & 34,47 & 32,77 & 869 \\
\hline MAN & 0,0731 & 0,0970 & 669 & 29,87 & 28,27 & 869 \\
\hline Metro & 0,0736 & 0,0882 & 685 & 29,57 & 26,84 & 869 \\
\hline $\mathrm{K}+\mathrm{S}$ & 0,0738 & 0,0883 & 834 & 27,51 & 25,05 & 869 \\
\hline Münchener Rück & 0,0763 & 0,0761 & 834 & 21,79 & 19,66 & 869 \\
\hline Volkswagen Vz. & 0,0765 & 0,0833 & 834 & 34,09 & 32,05 & 869 \\
\hline Linde & 0,0769 & 0,0780 & 834 & 20,42 & 19,57 & 869 \\
\hline Deutsche Post & 0,0781 & 0,0977 & 814 & 23,74 & 22,39 & 869 \\
\hline HeidelbergCement & 0,0808 & 0,1039 & 696 & 36,39 & 35,25 & 869 \\
\hline Fresenius & 0,0859 & 0,1246 & 814 & 19,88 & 19,63 & 869 \\
\hline Lufthansa & 0,0966 & 0,1167 & 834 & 30,13 & 28,47 & 869 \\
\hline Salzgitter & 0,1027 & 0,1116 & 118 & 35,03 & 31,77 & 869 \\
\hline Deutsche Telekom & 0,1063 & 0,1124 & 834 & 21,58 & 18,46 & 869 \\
\hline Infinion & 0,1646 & 0,1789 & 814 & 37,90 & 35,40 & 869 \\
\hline Commerzbank & 0,1745 & 0,3221 & 834 & 43,71 & 39,44 & 869 \\
\hline
\end{tabular}

Diese Tabelle zeigt den Mittelwert, den Median sowie die Anzahl der täglichen Beobachtungen (N) der Geld-Brief-Spanne $\left(G B S_{A}\right)$ und der Volatilität (Volatilität) der Aktien. Der Beobachtungszeitraum ist vom 01.01.2010 bis zum 01.04.2013.

Aktie auf. Die Abweichung beträgt 0,32\% für den kurz- und 0,29\% für den langfristigen SSF. Insgesamt gibt es neun Unternehmen bei denen die mittlere Abweichung der langfristigen Kontrakte kleiner als die der kurzfristigen Kontrakte ist. Der kurzfristige SSF auf das Unternehmen Fresenius weißt mit 0,69\% die größte mittlere Abweichung auf. Die maximale mittlere Abweichung für die langfristigen Kontrakte liegt bei 0,71\% für den Commerzbank SSF. Der Median liegt allgemein unter dem Mittelwert, was auf eine rechtsschiefe Verteilung schließen lässt. Ausgenommen davon sind lediglich die Un- 
ternehmen Henkel und Salzgitter, für die nur eine geringe Anzahl an Beobachtungen verfügbar ist. Der gesamte Beobachtungszeitraum umfasst 869 Handelstage, jedoch sind die Geld-Brief-Spannen für Aktien und SSF nicht für jeden Zeitpunkt verfügbar. Für die Unternehmen Salzgitter, Heidelberg Cement, MAN und Metro verringert sich die Anzahl der Beobachtungen auch dadurch, dass sie nicht während des gesamten Untersuchungszeitraums im DAX30 gelistet waren. Für die Unternehmen Continental und Lanxess, welche gegen Ende des Beobachtungszeitraums in den DAX30 aufgenommen wurden, sind keine Beobachtungen verfügbar.

In Tabelle 4.2 sind deskriptive Statistiken der beiden modelltheoretisch motivierten Variablen $G B S_{A}$ und Volatilität abgebildet. Siemens ist mit einer mittleren Geld-BriefSpanne von 0,044\% die liquideste Aktie. Die illiquideren Aktien weisen Mittelwerte von ca. $0,1 \%$ auf. Die Commerzbank Aktie stellt mit einer Geld-Brief-Spanne im Mittel von 0,17\% und im Median von 0,32\% einen besonders illiquiden Ausreißer dar. Anhand der deskriptiven Statistiken zur Volatilität der Aktien, die im Mittel zwischen $18 \%$ und $43 \%$ p.a. liegt, wird deutlich, dass eine Marktphase ohne extreme Schwankungen betrachtet wird. Die letzte modelltheoretische Variable ist die Laufzeit. Dabei entfallen ca. $53 \%$ $(47 \%)$ der Beobachtung auf kurzfristige (langfristige) SSF-Kontrakte, wobei die durchschnittliche Restlaufzeit ca. 31 Tage für kurzfristige und ca. 62 Tage für langfristige Kontrakte beträgt.

\subsubsection{Determinanten von Futurespreisen}

Ziel dieses Abschnittes ist es die Determinanten der Preise am SSF-Markt zu identifizieren. Dazu wird zunächst die Bedeutung der aus dem Modell abgeleiteten Variablen für die Preisabweichungen am SSF-Markt untersucht. Die darauffolgende Analyse dient insbesondere dazu Friktionen, die nicht im Modell enthalten sind, empirisch zu erfassen und deren Auswirkungen auf die Preisabweichungen am SSF-Markt zu analysieren. Anhand von Kontrollvariablen werden die Auswirkungen der Friktionen zunächst separat analysiert. Im letzten Abschnitt werden die simultanen Effekte aller Kontrollvariablen in einer Regression untersucht. Besonderer Fokus der Untersuchung liegt dabei auf der Fragestellung, ob die Illiquidität ein relevanter Faktor für die Preisabweichungen am SSF-Markt ist. 


\subsubsection{Basisfall}

Zur Überprüfung der Preishypothesen werden Fixed-Effects Panelregressionen durchgeführt. Das Regressionsmodell hat die folgende Form:

$$
\text { abs_ABW } W_{C o C i, t}=\alpha_{i}+\beta x_{i, t}+\varepsilon_{i, t},
$$

mit abs_AB $W_{C o C i, t}$ als Absolutbetrag der relativen Abweichung vom Cost-of-CarryPreis des SSF auf die Aktie $i$ zum Zeitpunkt $t, \alpha_{i}$ ist der über die Zeit konstante fixe Effekt der Aktie $i, \beta$ ist der Zeilenvektor der Regressionskoeffizienten und $x_{i, t}$ ist der Spaltenvektor der erklärenden Variablen von Aktie $i$ zum Zeitpunkt $t$. Für den Fehlerterm $\varepsilon_{i, t}$ wird angenommen, dass dieser einen Mittelwert von null hat und unabhängig von den erklärenden Variablen $x_{i, t}$ ist. Anhand des Modells aus Abschnitt 3.2 konnten die Variablen $G B S_{A}$, Volatilität und Laufzeit als Faktoren, die die Preisabweichungen am SSF-Markt beeinflussen, identifiziert werden. Eine Panelregression mit diesen Variablen bildet den Basisfall für die empirische Analyse der Preishypothesen. Dabei handelt es sich um ein unbalanciertes Panel Set, da sich die Zusammensetzung des DAX30 Index während des Untersuchungszeitraums geändert hat und die Geld-Brief-Spanne nicht für alle Aktien zu jedem Zeitpunkt zur Verfügung steht.

Die erste Spalte der Tabelle 4.3 zeigt die Ergebnisse der Basisregression. Die p-Werte sind in Klammern angegeben und basieren auf geclusterten Standardfehlern nach Petersen (2009). Es zeigt sich, dass sämtliche Variablen auf dem 1\% Niveau signifikant sind. Eine höhere Illiquidität am Aktienmarkt gemessen durch die Geld-Brief-Spanne sowie eine längere Restlaufzeit haben eine größere Abweichung von den Cost-of-Carry-Preisen zur Folge und stützen damit die modelltheoretischen Vorhersagen. Die Volatilität dagegen besitzt nicht das erwartete Vorzeichen. Dem Modell nach sollte eine steigende Volatilität zu kleineren Preisabweichungen führen. Empirisch zeigt sich jedoch, dass ein steigendes Risiko größere Preisabweichungen zur Folge hat. Dieses Ergebnis ist konsistent mit den empirischen Befunden aus der Literatur zu Fehlbewertungen von Indexfutures (vgl. z.B. Yadav \& Pope (1994)). Wie bereits in Abschnitt 4.2.2 erläutert, resultiert der negative Einfluss der Volatilität im Modell aus der sinkenden spekulativen Komponente des Market Makers. Es kann jedoch nicht belegt werden, dass Market Maker riskante Positionen zu spekulativen Zwecken halten. Dagegen ist es plausibel, dass eine hohe Volatilität die Bewertung von Futures in der Realität erschwert.

Eine der zentralen Fragestellungen im Rahmen der Preishypothesen ist, ob sich ein po- 
Tabelle 4.3: Determinanten der Abweichungen von den Cost-of-Carry-Preisen: Basisfall

\begin{tabular}{lclc}
\hline & $a b s \_A B W_{C o C}$ & & $a b s \_A B W_{C o C}$ \\
\hline$G B S_{A}$ & $0,536^{* * *}$ & Amihud $_{A}$ & $1,367^{* * *}$ \\
& $(0,000)$ & & $(0,000)$ \\
Volatilität & $0,007^{* * *}$ & Volatilität & $0,007^{* * *}$ \\
& $(0,000)$ & & $(0,000)$ \\
Laufzeit & $0,009^{* * *}$ & Laufzeit & $0,014^{* * *}$ \\
& $(0,003)$ & & $(0,000)$ \\
Konstante & $0,213^{* * *}$ & Konstante & $0,220^{* * *}$ \\
& $(0,000)$ & & $(0,000)$ \\
\hline Beobachtungen & 31.672 & & 34.828 \\
within $-R^{2}$ & 0,0564 & & 0,0595 \\
\hline
\end{tabular}

Die Tabelle zeigt die Ergebnisse der Fixed-Effects Panelregressionen mit dem Absolutbetrag der relativen Abweichungen der SSF-Midquotes von den Cost-of-Carry-Preisen ( $\left.a b s \_A B W_{C o C}\right)$ als abhängiger Variable. Die erklärenden Variablen in der ersten Regression sind $G B S_{A}$, Volatilität und Laufzeit. Die erklärenden Variablen in der zweiten Regression sind Amihud $_{A}$, Volatilität und Laufzeit. Der als Konstante ausgewiesene Wert ist der mean fixed effect. Die p-Werte sind in Klammern angegeben und wurden bestimmt durch geclusterte Standardfehler. Der Beobachtungszeitraum ist vom 01.01.2010 bis zum 01.04.2013. ***,**, und * geben die statistische Signifikanz auf dem $1 \%, 5 \%$, and $10 \%$ Niveau an.

sitiver Zusammenhang zwischen der Friktion eines illiquiden Aktienmarktes und den Preisabweichungen am SSF-Markt nachweisen lässt. Aus diesem Grund wird der Basisfall zusätzlich mit einem anderen Liquiditätsmaß geschätzt. Als alternatives Liquiditätsmaß wird ein Preiseinflussmaß verwendet, da es im Gegensatz zur Geld-Brief-Spanne nicht nur die Engekomponente sondern auch die Tiefekomponente der Liquidität berücksichtigt. Nach Goyenko et al. (2009) ist das Amihud-Maß am besten geeignet, um den Preiseinfluss zu erfassen, und wird deshalb in der Analyse verwendet. Das Maß wird als rollierender Durchschnitt der letzten 30 Handelstage nach Gleichung (2.1) berechnet und als $\mathrm{Amihud}_{A}$ bezeichnet. Zur Gewährleistung der Stationarität wird das Maß analog zum Vorgehen in der Studie von Acharya \& Pedersen (2005) mit dem Quotienten aus der aktuellen DAX30 Marktkapitalisierung und der DAX30 Marktkapitalisierung zu Beginn des Untersuchungszeitraums multipliziert. Sämtliche zur Berechnung benötigten Daten sind über Datastream verfügbar. Die Ergebnisse der Regression mit der Variable Amihud $_{A}$ als Liquiditätsmaß sind in der zweiten Spalte der Tabelle 4.3 abgebildet. Die Liquidität, gemessen durch das Amihud-Maß, ist ebenfalls hoch signifikant und besitzt das erwartete Vorzeichen. Auch die Vorzeichen und Signifikanzen der Variablen Volatilität und Laufzeit bleiben unverändert. Da der Fokus der Preishypothesen auf der Illiquidität des Aktienmarktes liegt, wird diese Robustheitsanalyse für alle folgenden Modellspezifikationen durchgeführt. 


\subsubsection{Alternative Risikomaße}

Ein Standardargument in der Finanzwirtschaft ist, dass nur das systematische Risiko und nicht das Gesamtrisiko von Relevanz ist, da das unsystematische Risiko durch Diversifikation eliminiert werden kann. Dieses Argument könnte auch für die Market Maker im SSF-Markt zutreffen, wenn diese durch das Handeln in verschiedenen Aktien zumindest ein gewisses Level an Diversifikation erreichen. Als erster Robustheitstest wird daher in den Regressionen der Tabelle 4.4 Panel A die Variable Volatilität durch das systematische Preisrisiko (Syst_Risiko) ersetzt. Die Variable Syst_Risiko wird dabei als Kovarianz der täglichen Aktienrenditen mit den täglichen DAX30 Indexrenditen über ein rollierendes Fenster der letzten 30 Handelstage berechnet. Zwischen dem systematischen Preisrisiko und den Abweichungen von den Cost-of-Carry-Preisen lässt sich ebenfalls ein signifikanter positiver Zusammenhang nachweisen. Insbesondere werden die Vorzeichen und Signifikanzen der Variablen $G B S_{A}$ und Laufzeit nicht durch die andere Risikomessung beeinflusst. Auch in der Regression, die die Variable Amihud $_{A}$ als Liquiditätsmaß verwendet, kann der positive Zusammenhang zwischen dem systematischen Preisrisiko und den Abweichungen von den Cost-of-Carry-Preisen nachgewiesen werden. Der signifikante Einfluss der Variablen Amihud $_{A}$ und Laufzeit auf die Preisabweichungen am SSF-Markt bleibt ebenfalls bestehen. Die Ergebnisse der Basisregression können somit als robust gegenüber diesem alternativen Risikomaß angesehen werden.

Neben dem Preisrisiko könnten die Preisabweichungen am SSF-Markt auch von anderen Risikofaktoren beeinflusst werden. So könnte eine im Zeitablauf stark schwankende Liquidität für den Market Maker ein zusätzliches Risiko bedeuten. Die Variation der Liquidität am Aktienmarkt wird im Folgenden als Liquiditätsrisiko bezeichnet. Das Modell in Abschnitt 3.2 macht keine Aussage bzgl. der Auswirkungen eines Liquiditätsrisikos auf die Preisabweichungen am SSF-Markt. Eine plausible Hypothese im Kontext des Verhaltens von Market Makern bei Friktionen ist, dass ein hohes Liquiditätsrisiko mit einer größeren Abweichung vom No-Arbitrage Preis einhergeht, da eine stark schwankende Liquidität am Aktienmarkt die Umsetzung einer Arbitragestrategie erschwert. In Panel B von Tabelle 4.4 wird die Basisregression um eine zusätzliche Variable, die das Liquiditätsrisiko am Aktienmarkt erfasst, erweitert. In der ersten Regression wird das Liquiditätsrisiko ( LiqRisiko $_{G B S}$ ) als die annualisierte Standardabweichung der relativen Geld-Brief-Spanne am Aktienmarkt für ein rollierendes Fenster der letzten 30 Handelstage geschätzt. Durch die Aufnahme dieser Kontrollvariable soll außerdem untersucht werden, ob die Effekte der modelltheoretischen Variablen robust gegenüber der 
Tabelle 4.4: Determinanten der Abweichungen von den Cost-of-Carry-Preisen: Alternative Risikomaße

Panel A: Systematisches Risiko

\begin{tabular}{lccc}
\hline & $a b s \_A B W_{C o C}$ & & $a b s \_A B W_{C o C}$ \\
\hline GBS $S_{A}$ & $0,559^{* * *}$ & Amihud & $1,446^{* * *}$ \\
Syst_Risiko & $(0,000)$ & & $(0,000)$ \\
& $0,037^{* * *}$ & Syst_Risiko & $0,038^{* * *}$ \\
Laufzeit & $(0,000)$ & & $(0,000)$ \\
& $0,009^{* * *}$ & Laufzeit & $0,014^{* * *}$ \\
Konstante & $(0,005)$ & & $(0,001)$ \\
& $0,352^{* * *}$ & Konstante & $0,361^{* * *}$ \\
& $(0,000)$ & & $(0,000)$ \\
\hline \multirow{2}{*}{ Beobachtungen } & 31.672 & & 34.828 \\
within- $R^{2}$ & 0,0477 & & 0,0488 \\
\hline
\end{tabular}

Panel B: Liquiditätsrisiko

\begin{tabular}{lccc}
\hline & $a b s \_A B W_{C o C}$ & & $a b s \_A B W_{C o C}$ \\
\hline$G B S_{A}$ & $0,540^{* * *}$ & Amihud $_{A}$ & $1,161^{* * *}$ \\
& $(0,000)$ & & $(0,000)$ \\
Volatilität & $0,007^{* * *}$ & Volatilität & $0,007^{* * *}$ \\
& $(0,000)$ & Laufzeit & $(0,000)$ \\
Laufzeit & $0,009^{* * *}$ & & $0,014^{* * *}$ \\
& $(0,003)$ & LiqRisiko Amihud & $(0,000)$ \\
LiqRisiko & & $0,713^{* *}$ \\
& $-0,006$ & Konstante & $(0,032)$ \\
Konstante & $(0,798)$ & & $0,221^{* * *}$ \\
& $0,214^{* * *}$ & & $(0,000)$ \\
\hline Beobachtungen & $(0,000)$ & & 34.828 \\
within- $R^{2}$ & 31.672 & & 0,0609 \\
\hline
\end{tabular}

Die Tabelle zeigt die Ergebnisse der Fixed-Effects Panelregressionen mit dem Absolutbetrag der relativen Abweichungen der SSF-Midquotes von den Cost-of-Carry-Preisen ( $a b s \_A B W_{C o C}$ ) als abhängiger Variable. Die erklärenden Variablen in der ersten Regression sind $G B S_{A}$, Syst_Risiko (Panel A), Volatilität (Panel B), Liq_Risiko GBS (Panel B) und

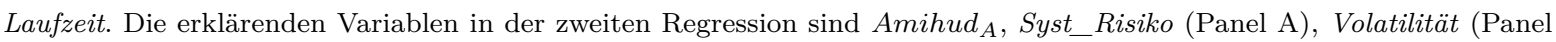
B), Liq_RisikoAmihud (Panel B) und Laufzeit. Der als Konstante ausgewiesene Wert ist der mean fixed effect. Die pWerte sind in Klammern angegeben und wurden bestimmt durch geclusterte Standardfehler. Der Beobachtungszeitraum ist vom 01.01.2010 bis zum 01.04.2013. ***, **, und * geben die statistische Signifikanz auf dem $1 \%, 5 \%$, and $10 \%$ Niveau an.

Berücksichtigung des Liquiditätsrisikos sind. Die erste Regression zeigt, dass die Vorzeichen und Signifikanzen der modelltheoretischen Variablen durch die Aufnahme der Variable LiqRisiko ${ }_{G B S}$ unverändert bleiben. Ein signifikanter Zusammenhang zwischen LiqRisiko $_{G B S}$ und den Preisabweichungen am SSF-Markt kann jedoch nicht nachgewiesen werden. Die Berechnung des in der zweiten Regression verwendeten Liquiditätsrisikos

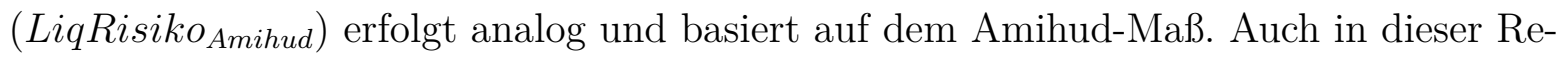


gression bleiben die Effekte der modelltheoretischen Variablen unverändert. Als zentrales Resultat lässt sich damit festhalten, dass unabhängig davon, wie Illiquidität und Liquiditätsrisiko gemessen werden, die modelltheoretischen Variablen in einem signifikanten und positiven Zusammenhang zu den Preisabweichungen am SSF-Markt stehen. Die Variable LiqRisiko $_{\text {Amihud }}$ selbst hat einen positiven Einfluss auf die Preisabweichungen am SSF-Markt und ist auf dem 5\% Niveau signifikant. Die Evidenz für einen positiven Zusammenhang von Liquiditätsrisiko und Preisabweichungen am SSF-Markt kann damit als schwach bezeichnet werden.

\subsubsection{Dividendeneffekte}

Bialkowski \& Jakubowski (2012) dokumentieren in ihrer Studie einen Anstieg des Handelsvolumens von SSF um den Ex-Tag. Dies sei darauf zurückzuführen, dass Investoren aufgrund der Besteuerung von Dividenden in der Zeit des Halbeinkünfteverfahrens Anreize hätten, Dividendenzahlungen mit Hilfe von SSF zu umgehen. Im Folgenden wird zunächst untersucht, ob vergleichbare Anreize auch in der Zeit nach Abschaffung des Halbeinkünfteverfahrens existieren. Darüber hinaus wird überprüft, ob das Handelsvolumen der SSF weiterhin um den Ex-Tag ansteigt, da eine zusätzliche Nachfrage nach SSF Auswirkungen auf die Preisabweichungen am SSF-Markt haben könnte.

In Tabelle 4.5 wird die Dividendenbesteuerung eines privaten, konfessionslosen Investors sowie einer Kapitalgesellschaft während der Zeit des Untersuchungszeitraums dargestellt. Der private Investor ist mit einem persönlichen Steuersatz von über $25 \%$ und einer Beteiligungsquote von unter $1 \%$ am Unternehmen der maximalen Dividendenbesteuerung ausgesetzt. Die Kapitalgesellschaft dagegen unterliegt mit einer Beteiligungsquote von über $15 \%$ am Unternehmen der geringsten Dividendenbesteuerung. Nach der Abgeltungssteuer werden die Einkünfte aus Kapitalerträgen von privaten Investoren mit maximal $25 \%$ besteuert. Unter Berücksichtigung des Solidaritätszuschlags (SolZ) verbleiben dem privaten Investor 73,625 \% einer Dividendenzahlung. Ist der aus der Dividendenzahlung resultierende prozentuale Kursabschlag größer als 73,625 \%, hat der private Investor einen Anreiz die Dividendenzahlung zu umgehen, indem er seine Aktie vor dem Ex-Tag verkauft, diese nach dem Ex-Tag zurückkauft und das zwischenzeitliche Preisexposure mit einer SSF-Longposition absichert. Zur Berechnung dieses Kursabschlags wird die Differenz von Schlusskurs vor dem Ex-Tag und Eröffnungskurs am Ex-Tag durch den Schlusskurs vor dem Ex-Tag geteilt. Der Kursabschlag für alle Dividendenzahlungen 
Tabelle 4.5: Dividendenbesteuerung

\begin{tabular}{lclc}
\hline & privater Investor & Kapitalgesellschaft \\
\hline Dividendenzahlung & 100 & & 100 \\
ESt $(>25 \%)$ & 25 & KSt $(15 \%$ auf $5 \%)$ & 0,75 \\
SolZ $(5,5 \%)$ & 1,375 & GewSt $(14 \%$ auf $5 \%)$ & 0,70 \\
verbleibend nach Steuern & 73,625 & & 98,55 \\
effektive Dividendensteuer & $26,375 \%$ & & $1,45 \%$ \\
\hline
\end{tabular}

Diese Tabelle zeigt die Besteuerung von Dividenden für einen privaten, konfessionslosen Investor und eine Kapitalgesellschaft in der Zeit vom 01.01.2010 bis zum 01.04.2013. Der private Investor hat eine Beteiligungsquote am Unternehmen von unter $1 \%$ der Marktkapitalisierung. Für die Kapitalgesellschaft liegt die Beteiligungsquote am Unternehmen bei über $15 \%$ der Marktkapitalisierung.

Tabelle 4.6: SSF Handelsvolumen (01.01.2010 - 01.04.2013)

\begin{tabular}{lccc}
\hline & \multicolumn{3}{c}{ Mittelwert Handelsvolumen (in Stück) } \\
\cline { 2 - 4 } & vollständiger Zeitraum & 40 Tage um Ex-Tag & 10 Tage um Ex-Tag \\
\hline kurzfristige SSF & 2.288 & 14.669 & 47.261 \\
langfristige SSF & 295 & 1.279 & 4.202 \\
\hline
\end{tabular}

Diese Tabelle zeigt das mittlere Handelsvolumen der kurz- und langfristigen SSF in Stück für den vollständigen Beobachtungszeitraum, ein symmetrisches Fenster von 40 sowie 10 Tagen um den Ex-Dividendentag. Der Beobachtungszeitraum ist vom 01.01.2010 bis zum 01.04.2013.

im Untersuchungszeitraum liegt im Median bei 82,808\%. Folglich wird der private Investor überwiegend nicht ausreichend für den entstehenden Kursabschlag entschädigt. Private Investoren bilden damit eine Gruppe, die Anreize hat, Dividendenzahlungen $\mathrm{zu}$ vermeiden und zu diesem Zweck möglicherweise verstärkt um den Ex-Tag herum SSF handeln. Für die Kapitalgesellschaft mit einer deutlich geringeren Steuerbelastung besteht dagegen die Möglichkeit durch den Kauf einer Aktie und dem Eingehen einer SSF-Shortposition vor dem Ex-Tag sowie dem Verkauf der Aktie und dem Glattstellen der SSF-Position nach dem Ex-Tag ihren Gewinn zu erhöhen.

Tabelle 4.6 zeigt, dass SSF auch in der Zeit vom 01.01.2010 bis zum 01.04.2013 verstärkt um den Ex-Tag herum gehandelt wurden. Das mittlere Handelsvolumen über den gesamten Beobachtungszeitraum liegt bei 2.288 Kontrakten pro Tag für kurzfristige SSF. Das mittlere Handelsvolumen für den Zeitraum von 10 Tagen um den Ex-Tag liegt dagegen mit 47.261 Kontrakten pro Tag für kurzfristige SSF wesentlich höher. Das erhöhte Handelsvolumen um die Dividendenzahlung lässt sich ebenfalls für einen Zeitraum von 40 Tagen um den Ex-Tag sowie für langfristige SSF-Kontrakte nachweisen. Insbesondere 
Tabelle 4.7: Determinanten der Abweichungen von den Cost-of-Carry-Preisen: Dividenden

Panel A: Dividenden 10 Tage

\begin{tabular}{lccc}
\hline & $a b s \_A B W_{C o C}$ & $a b s \_A B W_{C o C}$ \\
\hline$G B S_{A}$ & $0,537^{* * *}$ & Amihud & $1,375^{* * *}$ \\
& $(0,000)$ & & $(0,000)$ \\
Volatilität & $0,007^{* * *}$ & Volatilität & $0,007^{* * *}$ \\
& $(0,000)$ & Laufzeit & $(0,000)$ \\
Laufzeit & $0,010^{* * *}$ & & $0,014^{* * *}$ \\
& $(0,000)$ & Dividende $(10$ Tage $)$ & $(0,000)$ \\
Dividende (10 Tage) & $0,116^{* * *}$ & & $0,110^{* * *}$ \\
& $(0,002)$ & Konstante & $0,000)$ \\
Konstante & $0,208^{* * *}$ & & $0,216^{* * *}$ \\
& $(0,000)$ & & $(0,000)$ \\
\hline Beobachtungen & 31.672 & & 34.828 \\
within- $R^{2}$ & 0,0584 & 0,0612 \\
\hline
\end{tabular}

Panel B: Dividenden 40 Tage

\begin{tabular}{lccc}
\hline & $a b s \_A B W_{C o C}$ & & $a b s \_A B W_{C o C}$ \\
\hline$G B S_{A}$ & $0,539^{* * *}$ & Amihud $_{A}$ & $1,385^{* * *}$ \\
& $(0,000)$ & & $(0,000)$ \\
Volatilität & $0,007^{* * *}$ & Volatilität & $0,007^{* * *}$ \\
& $(0,000)$ & Laufzeit & $(0,000)$ \\
Laufzeit & $0,009^{* * *}$ & & $0,014^{* * *}$ \\
& $(0,003)$ & Dividende (40 Tage) & $(0,001)$ \\
Dividende (40 Tage) & $0,035^{* * *}$ & Konstante & $0,031^{* * *}$ \\
& $(0,003)$ & & $(0,008)$ \\
Konstante & $0,207^{* * *}$ & & $0,216^{* * *}$ \\
& $(0,000)$ & & $(0,000)$ \\
\hline Beobachtungen & 31.672 & & 34.828 \\
within- $R^{2}$ & 0.0570 & 0,0597 \\
\hline
\end{tabular}

Die Tabelle zeigt die Ergebnisse der Fixed-Effects Panelregressionen mit dem Absolutbetrag der relativen Abweichungen der SSF-Midquotes von den Cost-of-Carry-Preisen $\left(a b s \_A B W_{C o C}\right)$ als abhängiger Variable. Die erklärenden Variablen in der ersten Regression sind $G B S_{A}$, Volatilität, Dividende 10 Tage (Panel A), Dividende 40 Tage (Panel B) und Laufzeit. Die erklärenden Variablen in der zweiten Regression sind Amihud $_{A}$, Volatilität, Dividende 10 Tage (Panel A), Dividende 40 Tage (Panel B) und Laufzeit. Der als Konstante ausgewiesene Wert ist der mean fixed effect. Die p-Werte sind in Klammern angegeben und wurden bestimmt durch geclusterte Standardfehler. Der Beobachtungszeitraum ist vom 01.01.2010 bis zum 01.04.2013. ${ }^{* *},{ }^{* *}$, und ${ }^{*}$ geben die statistische Signifikanz auf dem $1 \%, 5 \%$, and $10 \%$ Niveau an.

existiert auch auf Basis der einzelnen SSF kein Fall, für den das mittlere Handelsvolumen des gesamten Untersuchungszeitraums größer als das um den Ex-Tag war.

Aufgrund des erhöhten Handelsvolumens um den Ex-Tag wird in einer neuen Modellspezifikation untersucht, ob diese zusätzliche Nachfrage nach SSF einen Einfluss auf die 
Preisabweichungen am SSF-Markt hat. Ist die Überschussnachfrage um den Ex-Tag ungleich null und haben die Market Maker Informationen darüber, ob es sich dabei um eine Nachfrage nach Long- oder Shortpositionen handelt, stehen aber nicht im vollständigen Wettbewerb, können sie die zusätzliche Nachfrage ausnutzen, um ihren Profit zu erhöhen. ${ }^{4}$ Besteht eine Überschussnachfrage nach Long- bzw. Shortpositionen, können die Markt Maker den Geld- bzw. Briefkurs so setzen, dass die nachgefragte Position teurer wird. Das Einseitige verschieben des Geld- bzw. Briefkurses würde zu einer größeren Abweichung des Midquotes vom Cost-of-Carry-Preis führen.

Die Auswirkungen des gestiegenen Handelsvolumens auf die Preisabweichungen am SSFMarkt werden mit Hilfe einer Dummy Variablen untersucht. Diese hat den Wert Eins für ein symmetrisches Fenster von 10 Tagen um den Ex-Tag und sonst den Wert null (Dividende 10 Tage). Die Ergebnisse unter Einbeziehung dieser Kontrollvariable sind in Tabelle 4.7 Panel A dargestellt. Die Variable Dividende 10 Tage ist hoch signifikant und hat das erwartete positive Vorzeichen. Dieses Resultat ist unabhängig davon, ob die Illiquidität am Aktienmarkt durch die Geld-Brief-Spanne oder das Amihud-Maß gemessen wird. Zur Überprüfung der Robustheit dieser Ergebnisse wird in Panel B eine Dummy Variable verwendet, die den Wert Eins für ein symmetrisches Fenster von 40 Tagen um den Ex-Tag annimmt (Dividende 40 Tage). Der mit dem unvollständigen Wettbewerb zwischen den Markt Makern konsistente positive Einfluss kann auch für diese Kontrollvariable bestätigt werden. Insbesondere zeigt sich, dass sich in beiden Fällen weder die Signifikanzen noch die Vorzeichen der modelltheoretischen Variablen verändern. Die Robustheit des Basisfalls kann folglich erneut dokumentiert werden.

\subsubsection{Effekte von Finanzierungskosten}

Der zentrale Aspekt der modelltheoretischen Analyse in Kapitel 3 war die Ableitung empirisch testbarer Hypothesen zu den Preis- und Liquiditätseffekten auf dem Futuresmarkt. Zu diesem Zweck wurde die Illiquidität sowohl auf dem Aktien- als auch auf dem Futuresmarkt modelliert. Die Modellierung der Kosten, die bei der Finanzierung über den Anleihemarkt entstehen, war dagegen nicht Teil der Untersuchung. Vereinfachend wurde angenommen, dass der Market Maker sich zu einem konstanten Zinssatz am Anleihemarkt risikolos finanzieren kann.

\footnotetext{
${ }^{4}$ Baule (2011) zeigt, dass Market Maker im Fall von Diskont-Zertifikaten zusätzliche Nachfrage antizipieren und ausnutzen.
} 
Tabelle 4.8: Determinanten der Abweichungen von den Cost-of-Carry-Preisen: Finanzierungskosten

\begin{tabular}{|c|c|c|c|}
\hline & $a b s \_A B W_{C o C}$ & & $a b s \_A B W_{C o C}$ \\
\hline$G B S_{A}$ & $\begin{array}{l}0,535^{* * *} \\
(0,000)\end{array}$ & $\operatorname{Amihud}_{A}$ & $\begin{array}{l}1,446^{* * *} \\
(0,000)\end{array}$ \\
\hline Volatilität & $\begin{array}{l}0,006^{* * *} \\
(0,000)\end{array}$ & Volatilität & $\begin{array}{l}0,006^{* * *} \\
(0,000)\end{array}$ \\
\hline Laufzeit & $\begin{array}{l}0,007^{* *} \\
(0,029)\end{array}$ & Laufzeit & $\begin{array}{l}0,011^{* * *} \\
(0,004)\end{array}$ \\
\hline$L I B O R$ & $\begin{array}{l}0,070^{* * *} \\
(0,000)\end{array}$ & $L I B O R$ & $\begin{array}{l}0,077^{* * *} \\
(0,000)\end{array}$ \\
\hline Konstante & $\begin{array}{l}0,184^{* * *} \\
(0,000)\end{array}$ & Konstante & $\begin{array}{l}0,187^{* * *} \\
(0,000)\end{array}$ \\
\hline $\begin{array}{l}\text { Beobachtungen } \\
\text { within }-R^{2}\end{array}$ & $\begin{array}{l}31.672 \\
0,0591\end{array}$ & & $\begin{array}{l}34.828 \\
0,0624\end{array}$ \\
\hline
\end{tabular}

Die Tabelle zeigt die Ergebnisse der Fixed-Effects Panelregressionen mit dem Absolutbetrag der relativen Abweichungen der SSF-Midquotes von den Cost-of-Carry-Preisen $\left(a b s \_A B W_{C o C}\right)$ als abhängiger Variable. Die erklärenden Variablen in der ersten Regression sind $G B S_{A}$, Volatilität, Laufzeit und LIBOR. Die erklärenden Variablen in der zweiten Regression sind Amihud $_{A}$, Volatilität, Laufzeit und LIBOR. Der als Konstante ausgewiesene Wert ist der mean fixed effect. Die p-Werte sind in Klammern angegeben und wurden bestimmt durch geclusterte Standardfehler. Der Beobachtungszeitraum ist vom 01.01.2010 bis zum 01.04.2013. ***,**, und * geben die statistische Signifikanz auf dem $1 \%, 5 \%$, and $10 \%$ Niveau an.

Ziel dieses Abschnittes ist es nun in der empirischen Analyse für im Zeitablauf schwankenden Finanzierungskosten zu kontrollieren und die Auswirkungen auf die Preisabweichungen am SSF-Markt zu untersuchen. Die Market Maker sind aufgrund der Margin Anforderungen an ihre SSF-Position Kosten, die bei der Kapitalbeschaffung über den Anleihemarkt entstehen, ausgesetzt. Halten sie eine Longposition (Shortposition) in SSF, werden sie eine Geld-Brief-Spanne stellen, so dass der Midquote unter (über) dem Costof-Carry-Preis liegt. Dadurch setzen sie Anreize, dass Investoren SSF kaufen (verkaufen) und ihre eigene SSF-Position sowie die damit verbundenen Kosten sinken. Sind die Kosten der Finanzierung hoch, werden sie stärkere Anreize setzen. Folglich ist bei hohen Finanzierungskosten eine größere Abweichung vom Cost-of-Carry-Preis zu erwarten.

Die Kosten der Finanzierung werden in dieser Arbeit durch den Drei-Monats-LIBOR Zinssatz $(L I B O R)$ approximiert. In Tabelle 4.8 wird der Basisfall um die Variable LIBOR erweitert, um die Auswirkungen schwankender Finanzierungskosten empirisch zu analysieren. Die Messung der Finanzierungskosten ist mit einer gewissen Problematik behaftet, da der Beobachtungszeitraum bereits in einer Phase der expansiven Geldpolitik und damit geringer Zinsen liegt. Die Signifikanz und das positive Vorzeichen der Va- 
riable $L I B O R$ sind konsistent mit der vorangegangenen Überlegung und bestätigen den positiven Einfluss der Finanzierungskosten auf die Preisabweichungen am SSF-Markt. Ein weiteres Ziel dieser Robustheitsanalyse ist zu überprüfen, ob die Vorzeichen und Signifikanzen der modelltheoretischen Variablen trotz dieser Kontrollvariablen unverändert bleiben. Es zeigt sich, dass die Illiquidität unabhängig von ihrer Messung weiterhin ein relevanter Faktor für die Preisabweichungen am SSF-Markt ist. Auch die übrigen modelltheoretischen Variablen werden nicht durch die Aufnahme der Variable LIBOR beeinflusst.

\subsubsection{Futuresliquidität}

Eine weitere Friktion, die die Abweichungen von den Cost-of-Carry-Preisen beeinflussen könnte und deren Auswirkungen nicht direkt im Modell untersucht werden können, ist Illiquidität des Futuresmarktes selbst. Die Optionsliteratur findet Evidenz, dass die Illiquidität am Terminmarkt ein Faktor ist, der die Preise am Terminmarkt beeinflusst (vgl. z. B. Chou et al. (2011)). Auch am SSF-Markt könnte die Illiquidität Auswirkungen auf die Abweichungen von den Cost-of-Carry-Preisen haben. Liegt eine Preisabweichung eines SSF vor, würde die Illiquidität am SSF-Markt das Umsetzen einer Arbitragestrategie und damit die Bewegung zum No-Arbitrage-Preis erschweren. Folglich ist bei einer hohen (geringen) Illiquidität am SSF-Markt eine hohe (geringe) Abweichung vom Costof-Carry-Preis zu erwarten.

Die Auswirkungen dieser Friktion werden untersucht, indem die Illiquidität des SSFMarktes gemessen durch die Geld-Brief-Spanne $\left(G B S_{F}\right)$ als Kontrollvariable in die Analyse aufgenommen wird. Die Ergebnisse dieser Regressionen sind in Tabelle 4.9 abgebildet. Wie erwartet kann ein positiver und signifikanter Zusammenhang zwischen der Illiquidität am Terminmarkt und den Preisabweichungen am SSF-Markt nachgewiesen werden. Dieses Ergebnis ist unabhängig davon, wie die Illiquidität am Aktienmarkt gemessen wird. Beide Regressionen bestätigen darüber hinaus erneut die Robustheit der Ergebnisse des Basisfalls.

\subsubsection{Simultane Effekte}

In diesem Abschnitt werden die Preiseffekte unter Berücksichtigung aller Kontrollvariablen in einer Regression analysiert. Durch die simultane Analyse der Kontrollvariablen 
Tabelle 4.9: Determinanten der Abweichungen von den Cost-of-Carry-Preisen: Futuresilliquidität

\begin{tabular}{|c|c|c|c|}
\hline & $a b s \_A B W_{C o C}$ & & $a b s \_A B W_{C o C}$ \\
\hline$G B S_{A}$ & $\begin{array}{l}0,489^{* * *} \\
(0,000)\end{array}$ & $\operatorname{Amihud}_{A}$ & $\begin{array}{l}1,282^{* * *} \\
(0,000)\end{array}$ \\
\hline Volatilität & $\begin{array}{l}0,006^{* * *} \\
(0,000)\end{array}$ & Volatilität & $\begin{array}{l}0,006^{* * *} \\
(0,000)\end{array}$ \\
\hline Laufzeit & $\begin{array}{l}0,005^{* * *} \\
(0,062)\end{array}$ & Laufzeit & $\begin{array}{l}0,010^{* * *} \\
(0,005)\end{array}$ \\
\hline$G B S_{F}$ & $\begin{array}{l}0,057^{* * *} \\
(0,000)\end{array}$ & $G B S_{F}$ & $\begin{array}{l}0,060^{* * *} \\
(0,000)\end{array}$ \\
\hline Konstante & $\begin{array}{l}0,174^{* * *} \\
(0,000)\end{array}$ & Konstante & $\begin{array}{l}0,178^{* * *} \\
(0,000)\end{array}$ \\
\hline $\begin{array}{l}\text { Beobachtungen } \\
\text { within } R^{2}\end{array}$ & $\begin{array}{l}31.672 \\
0,0847\end{array}$ & & $\begin{array}{l}34.828 \\
0,0927\end{array}$ \\
\hline
\end{tabular}

Die Tabelle zeigt die Ergebnisse der Fixed-Effects Panelregressionen mit dem Absolutbetrag der relativen Abweichungen der SSF-Midquotes von den Cost-of-Carry-Preisen $\left(a b s \_A B W_{C o C}\right)$ als abhängiger Variable. Die erklärenden Variablen in der ersten Regression sind $G B S_{A}$, Volatilität, Laufzeit und $G B S_{F}$. Die erklärenden Variablen in der zweiten Regression sind Amihud $_{A}$, Volatilität, Laufzeit und $G B S_{F}$. Der als Konstante ausgewiesene Wert ist der mean fixed effect. Die p-Werte sind in Klammern angegeben und wurden bestimmt durch geclusterte Standardfehler. Der Beobachtungszeitraum ist vom 01.01.2010 bis zum 01.04.2013. ***,**, und * geben die statistische Signifikanz auf dem $1 \%, 5 \%$, and $10 \%$ Niveau an.

soll ausgeschlossen werden, dass die Ergebnisse der Einzeleffekte durch die Nichtberücksichtigung von signifikanten Variablen verzerrt sind. Tabelle 4.10 zeigt die Ergebnisse der Regressionen unter Berücksichtigung aller Kontrollvariablen. Mit einer einzigen Ausnahme stimmen die Wirkungen und Signifikanzen der Variablen mit denen der Einzeleffekte überein. In den Regressionen sind weiterhin beide Variablen für die Illiquidität am Aktienmarkt positiv und signifikant. Auch die Effekte für die Volatilität am Aktienmarkt sowie für sämtliche Kontrollvariablen sind in beiden Regressionen unverändert gegenüber den Einzeleffekten. Lediglich die Variable Laufzeit ist in der Regression, die die Geld-Brief-Spanne als Liquiditätsmaß verwendet, insignifikant. Folglich ist es möglich die Unterschiede in den Preisabweichungen von kurz- und langfristigen SSF durch die anderen Variablen zu erklären. In der Regression, die das Amihud-Maß verwendet, ist die Variable Laufzeit auf dem 5\% Niveau signifikant. Insgesamt sind die Ergebnisse damit als sehr robust zu bezeichnen. 
Tabelle 4.10: Determinanten der Abweichungen von den Cost-of-Carry-Preisen: Simultane Effekte

\begin{tabular}{|c|c|c|c|}
\hline & $a b s \_A B W_{C o C}$ & & $a b s \_A B W_{C o C}$ \\
\hline$G B S_{A}$ & $\begin{array}{l}0,491^{* * *} \\
(0,000)\end{array}$ & $\operatorname{Amihud}_{A}$ & $\begin{array}{l}1,174^{* * *} \\
(0,000)\end{array}$ \\
\hline Volatilität & $\begin{array}{l}0,005^{* * *} \\
(0,000)\end{array}$ & Volatilität & $\begin{array}{l}0,005^{* * *} \\
(0,000)\end{array}$ \\
\hline Laufzeit & $\begin{array}{c}0,003 \\
(0,198)\end{array}$ & Laufzeit & $\begin{array}{l}0,008^{* *} \\
(0,018)\end{array}$ \\
\hline LiqRisiko $_{G B S}$ & $\begin{array}{l}-0,000 \\
(0,970)\end{array}$ & LiqRisiko $_{\text {Amihud }}$ & $\begin{array}{l}0,155^{* *} \\
(0,048)\end{array}$ \\
\hline Dividende (10 Tage) & $\begin{array}{l}0,100^{* * *} \\
(0,000)\end{array}$ & Dividende (10 Tage) & $\begin{array}{l}0,092^{\text {*** }} \\
(0,000)\end{array}$ \\
\hline LIBOR & $\begin{array}{l}0,058^{* * *} \\
(0,000)\end{array}$ & $L I B O R$ & $\begin{array}{l}0,064^{* * *} \\
(0,000)\end{array}$ \\
\hline$G B S_{F}$ & $\begin{array}{l}0,056^{* * *} \\
(0,000)\end{array}$ & $G B S_{F}$ & $\begin{array}{l}0,058^{* * *} \\
(0,000)\end{array}$ \\
\hline Konstante & $\begin{array}{l}0,147^{* * *} \\
(0,000)\end{array}$ & Konstante & $\begin{array}{l}0,148^{* * *} \\
(0,000)\end{array}$ \\
\hline $\begin{array}{l}\text { Beobachtungen } \\
\text { within- } R^{2}\end{array}$ & $\begin{array}{l}31.672 \\
0,0885\end{array}$ & & $\begin{array}{l}34.828 \\
0,0990\end{array}$ \\
\hline
\end{tabular}

Die Tabelle zeigt die Ergebnisse der Fixed-Effects Panelregressionen mit dem Absolutbetrag der relativen Abweichungen der SSF-Midquotes von den Cost-of-Carry-Preisen (abs_AB $\left.W_{C o C}\right)$ als abhängiger Variable. Die erklärenden Variablen in der ersten Regression sind $G B S_{A}$, Volatilität, Laufzeit, LiqRisiko $o_{B S}$, Dividende (10 Tage), LIBOR und $G B S_{F}$. Die erklärenden Variablen in der zweiten Regression sind Amihud $_{A}$, Volatilität, Laufzeit, LiqRisiko Amihud, Dividende (10 Tage), $L I B O R$ und $G B S_{F}$. Der als Konstante ausgewiesene Wert ist der mean fixed effect. Die p-Werte sind in Klammern angegeben und wurden bestimmt durch geclusterte Standardfehler. Der Beobachtungszeitraum ist vom 01.01.2010 bis zum 01.04.2013. ${ }^{* * *},{ }^{* *}$, und ${ }^{*}$ geben die statistische Signifikanz auf dem $1 \%, 5 \%$, and $10 \%$ Niveau an. 


\subsection{Liquiditätseffekte}

Der Aufbau des Abschnittes zur Überprüfung der Liquiditätseffekte erfolgt analog zudem der Preiseffekte. Zunächst wird die Datenbasis beschrieben und es werden die erwarteten Effekte erläutert. Deskriptive Statistiken der zentralen Variablen und die empirische Analyse der Liquiditätshypothesen bilden den zweiten und dritten Teil dieses Abschnittes.

\subsubsection{Variablen und erwartete Effekte}

Die Modellvariante aus Abschnitt 3.1 mit Liquiditätsrisiko erlaubt, Vorhersagen über die gesamte Preiseinflussfunktion am Futuresmarkt zu treffen. Anhand der Abbildungen 3.5 und 3.6 ist ersichtlich, dass die Liquiditätseffekte unter Berücksichtigung eines Liquiditätsrisikos stärker für (absolut) große Werte von $H_{*}$ sind. Dieses zentrale Resultat ist der Grund dafür, dass ein Liquiditätsmaß, das nur die Liquidität zum besten Geldund Briefkurs erfasst, zur Überprüfung der modelltheoretischen Liquiditätshypothesen nicht ausreichend ist. Hierfür wird daher ein Maß benötig, das die Liquidität im Orderbuch über den besten Geld- und Briefkurs hinaus abbildet. Der in Abschnitt 2.2.2 diskutierte CRT ist in der Lage, neben der Marktenge ebenfalls die Markttiefe zu erfassen, und erfüllt damit dieses Kriterium. Die Deutsche Börse folgt dem Konzept des CRT und entwickelte das Xetra Liquiditätsmaß ${ }^{5}$ (XLM), welches ebenfalls auf den Kosten einer gleichzeitigen Kauf- und Verkaufstransaktion basiert. Die Berechnung des XLM erfolgt anhand von sekündlichen Schnappschüssen des gesamten Orderbuches während der Handelszeiten. Dabei werden auch die nicht sichtbaren Aufträge, sogenannte Iceberg Orders ${ }^{6}$, berücksichtigt. In der empirischen Analyse werden tägliche Durchschnitte dieser hochfrequenten Daten verwendet. Das XLM gibt damit die durchschnittlichen täglichen Illiquiditätskosten in Prozent an, welche bei einem gleichzeitigen Kauf und Verkauf in Höhe eines bestimmten Eurobetrags entstehen würden. In der empirischen Studie werden zwei XLMs verwendet, die sich auf einen Betrag von 50.000 bzw. 100.000 Euro beziehen. Das XLM ist das ideale Maß für die empirische Überprüfung der aus

${ }^{5}$ Für eine ausführliche Abhandlung zum XLM sei auf Gomber \& Schweickert (2002) sowie Gomber et al. (2015) verwiesen.

6 Als Iceberg Order wird ein Kauf- oder Verkaufsauftrag bezeichnet, bei dem die übrigen Marktteilnehmer lediglich einen kleinen Teil des tatsächlichen Auftragsvolumens im offenen Orderbuch sehen können. 
dem Modell abgeleiteten Hypothesen, da es die Eigenschaften des ILM genauestens abbildet. Beide Maße geben die Illiquiditätskosten pro Einheit für eine gegebene Auftragsgröße an. Der einzige Unterschied besteht darin, dass das XLM die Größe des Auftrags in Euro und nicht wie das $I L M$ in Stück misst. Für die empirische Analyse ist die Formulierung in Euro geeigneter, da so die Vergleichbarkeit von Aktien und SSF mit unterschiedlichen Preisniveaus gewährleistet ist. Des Weiteren wird das $X L M$ von der deutschen Börse sowohl für SSF als auch für Aktien berechnet, was es erlaubt, die Illiquidität auf beiden Märkten konsistent zu erfassen. Die Illiquiditätsmaße für den Datensatz der DAX30 Aktien und SSF sind für den Zeitraum vom 01.01.2010 bis 31.12.2011 verfügbar. Neuere Daten des XLM können nicht bezogen werden, da die Deutsche Börse den Vertrieb der XLM Daten für SSF seit Ende 2011 eingestellt hat. Damit ergibt sich für die Überprüfung der Liquiditätshypothesen ein anderer Untersuchungszeitraum als für die Preishypothesen, da diese die SSF XLM Daten nicht benötigen.

Sämtliche Modellspezifikationen in der empirischen Analyse werden nicht nur mit dem XLM analysiert, sondern darüber hinaus mit der quotierten Geld-Brief-Spanne, welche als alternatives Liquiditätsmaß für ein XLM mit kleiner Auftragsgröße anzusehen ist. Dieses Vorgehen erfolgt aus zwei Gründen: Erstens ist die Geld-Brief-Spanne das in der akademischen Literatur am weitesten verbreitete Liquiditätsmaß und zweitens sollte das Liquiditätsrisiko dem Modell nach nur einen Einfluss auf die Illiquidität am Futuresmarkt für große Werte von $H_{*}$ haben, nicht jedoch für kleine Werte von $H_{*}$. Diese Vorhersage kann überprüft werden, indem die Ergebnisse für das $X L M$, das für große Werte von $H_{*}$ steht, mit denen für die Geld-Brief-Spanne, die für kleine Werte von $H_{*}$ steht, verglichen werden. Die Berechnung der relativen Geld-Brief-Spannen $(G B S)$ für den Zeitraum vom 01.01.2010 - 31.12.2011 erfolgt auf Basis des Datensatzes, der zur Überprüfung der Preishypothesen verwendet wurde.

Ziel der empirischen Analyse ist es, die Hypothesen bzgl. der Illiquiditätsübertragung vom Aktien- auf den Futuresmarkt zu überprüfen. Die abhängige Variable ist daher die Illiquidität des SSF-Markt. Die Variablen für die Illiquidität der SSF-Kontrakte werden mit $X L M_{F}$ und $G B S_{F}$ bezeichnet.

Das Modell sagt voraus, dass die Illiquidität des SSF-Marktes von der Illiquidität des Aktienmarktes abhängt. Daher wird die Illiquidität des Aktienmarktes gemessen durch das $X L M$ der Aktien $\left(X L M_{A}\right)$ und die relative Geld-Brief-Spanne der Aktien $\left(G B S_{A}\right)$ als erste erklärende Variable in der empirischen Analyse verwendet. Nach der „derivativehedge-theory" sollte die Illiquidität am Futuresmarkt mit steigender Illiquidität am Akti- 
enmarkt zunehmen. Sofern Aktien- und Futuresmarkt als komplementäre Märte gesehen werden, folgt diese Hypothese, da die Märkte aufgrund der Hedgingaktivität der Market Maker verbunden sind. Eine alternative Sichtweise ist, dass SSF als Substitute für Aktien eingesetzt werden könnten. SSF könnten ein attraktives Substitut darstellen, da sie weniger Kapitaleinsatz als Aktien benötigen. Werden SSF und Aktien als Substitute angesehen, wäre eine inverse Liquiditätsbeziehung zu erwarten.

Eine hohe Risikoaversion des Market Makers sowie eine hohe Volatilität der Aktienrenditen sollten dem Modell nach ebenfalls zu einem illiquideren SSF-Markt führen. Analog zum Vorgehen bei den Preishypothesen werden diese Faktoren nicht separat analysiert. Die annualisierte Standardabweichung der täglichen Aktienrenditen, geschätzt über ein rollierendes Fenster von 30 Handelstagen, wird erneut als zweite erklärende Variable verwendet und als Volatilität bezeichnet. Die Volatilität wirkt sich im Rahmen des Modells positiv auf die Illiquidität des Futuresmarktes aus, da der Market Maker bei einer hohen Volatilität einem größeren Risiko ausgesetzt ist. Es gibt jedoch Argumente außerhalb des modelltheoretischen Rahmens, die die entgegengesetzte Richtung erwarten lassen. Historisch betrachtet war die hohe Volatilität von Kassamärkten eine wesentliche Einflussgröße für die Entwicklung von großen und liquiden Futuresmärkten, um die steigende Nachfrage nach Hedginginstrumenten für die Kassapreisrisiken zu bedienen. ${ }^{7}$

Die dritte aus dem Modell abgeleitete erklärende Variable ist das Liquiditätsrisiko am Aktienmarkt. Gemessen wird das Liquiditätsrisiko als Standardabweichung der täglichen $X L M_{A}$ und $G B S_{A}$ Werte, geschätzt über ein rollierendes Fenster von 30 Handelstagen. Das Liquiditätsrisiko wird als LiqRisiko bzw. LiqRisiko $o_{G B S}$ bezeichnet und soll das Risiko reflektieren, dem der Market Maker aufgrund schwankender Illiquidität ausgesetzt ist. Ein höheres Liquiditätsrisiko erhöht die Kosten eines risikoaversen Market Makers und dementsprechend folgt aus dem Modell, dass ein steigendes Liquiditätsrisiko die Illiquidität am Futuresmarkt verstärken sollte. Nach der Substitutionshypothese wäre dagegen die entgegengesetzte Wirkung zu erwarten. Sind Aktien und SSF als Substitute anzusehen, könnte ein steigendes Liquiditätsrisiko am Aktienmarkt Investoren dazu veranlassen auf den Futuresmarkt zu wechseln, was mit einer höheren Liquidität an diesem Markt einhergehen würde.

Analog zum Vorgehen der empirischen Analyse der Preishypothesen werden die SSFKontrakte mit der kürzesten und zweitkürzesten Laufzeit für jede Aktie, die während

\footnotetext{
${ }^{7}$ Ein Beispiel stellt der Beginn des Handels von Crude Oil Futures an der New York Mercantile Ex-
} change im Jahr 1986 nach einer Periode von Preisturbulenzen an den weltweiten Ölmärkten dar. 
des Beobachtungszeitraums im DAX30 war, in die Analyse miteinbezogen. Die SSF auf das gleiche Basiswertpapier mit unterschiedlicher Laufzeit werden verwendet, um die aus dem Modell abgeleitete Hypothese bzgl. der Laufzeiteffekte zu überprüfen. Wie im Fall der Preishypothesen wird eine Dummy Variable (Laufzeit) verwendet, die den Wert 0 für Futures mit der kürzesten Laufzeit und 1 für Futures mit der zweitkürzesten Laufzeit annimmt. Dem Modell nach sollten langfristige Futures illiquider als kurzfristige Futures sein. Folglich ist ein positiver Einfluss der Dummy Variablen zu erwarten.

Die Korrelation zwischen dem Preisrisiko und dem Liquiditätsrisiko sowie die erwartete Preisveränderung beeinflussen dem Modell nach ebenfalls die Liquidität am Futuresmarkt. Beide Effekte hängen jedoch davon ab, ob im Aggregat eine Nachfrage nach Longoder Shortpositionen besteht. Weder das XLM noch die Geld-Brief-Spanne ermöglichen es, zwischen einem durch Kauf oder Verkauf initiierten Preiseinfluss zu unterscheiden. Folglich ist es nicht möglich, die modelltheoretischen Vorhersagen zur Korrelation von Preis- und Liquiditätsrisiko sowie zur erwarteten Preisveränderung empirisch zu überprüfen.

\subsubsection{Deskriptive Statistik}

Das entscheidende Maß für die empirische Analyse der Liquiditätshypothesen ist das $X L M$. Daher werden zunächst deskriptive Statistiken des XLM der Aktien und SSF dargestellt. Die Werte des XLM beziehen sich auf ein Volumen von 50.000 und 100.000 Euro. Das XLM wird in Prozent angegeben und entspricht den Kosten, die aus einem gleichzeitigen Kauf und Verkauf im Wert von 50.000 bzw. 100.000 Euro resultieren würden. Die Analyse basiert wie im Fall der Preishypothesen auf einem Datensatz bei dem Werte größer als 1000 BP gelöscht wurden. ${ }^{8}$

In Tabelle 4.11 ist der Mittelwert, der Median sowie die Anzahl der verfügbaren Beobachtungen für das $X L M_{A}$ im Zeitraum vom Januar 2010 bis Dezember 2011 für alle 31 in diesem Zeitraum im DAX30 gelisteten Aktien dargestellt. Der mittlere Preiseinfluss für die liquidesten Aktien liegt ca. bei 0,06\%, wobei das Unternehmen Siemens mit einem mittleren Preiseinfluss von 0,0524\% (0,059\%) für ein Volumen von 50.000 (100.000) Euro die liquideste Aktie besitzt. Die illiquidesten Aktien während des Beobachtungszeitraums sind Heidelberg Cement mit einem $X L M_{A}$ von $0,131 \%$ für ein

\footnotetext{
${ }^{8}$ Im Extrem existieren Beobachtungen von über 10.000 BP.
} 
Tabelle 4.11: Illiquiditätsmaße der Aktien

\begin{tabular}{|c|c|c|c|c|c|c|}
\hline & \multicolumn{6}{|c|}{ Illiquiditätsmaß Aktien (XLM $M_{A}$ in Prozent) } \\
\hline & \multicolumn{3}{|c|}{50.000 Euro } & \multicolumn{3}{|c|}{ 100.000 Euro } \\
\hline & Mittelwert & Median & $\mathrm{N}$ & Mittelwert & Median & $\mathrm{N}$ \\
\hline Siemens & 0,0524 & 0,0468 & 510 & 0,0590 & 0,0563 & 509 \\
\hline SAP & 0,0577 & 0,0500 & 510 & 0,0662 & 0,0611 & 509 \\
\hline Deutsche Telekom & 0,0594 & 0,0615 & 509 & 0,0694 & 0,0701 & 509 \\
\hline E.ON & 0,0613 & 0,0518 & 508 & 0,0697 & 0,0625 & 507 \\
\hline BASF & 0,0625 & 0,0565 & 510 & 0,0716 & 0,0684 & 509 \\
\hline Allianz & 0,0629 & 0,0577 & 509 & 0,0757 & 0,0699 & 509 \\
\hline RWE & 0,0646 & 0,0552 & 510 & 0,0759 & 0,0684 & 509 \\
\hline Deutsche Bank & 0,0646 & 0,0575 & 510 & 0,0792 & 0,0701 & 510 \\
\hline Daimler & 0,0661 & 0,0612 & 509 & 0,0812 & 0,0742 & 509 \\
\hline Bayer & 0,0669 & 0,0587 & 509 & 0,0757 & 0,0706 & 508 \\
\hline BMW & 0,0788 & 0,0749 & 510 & 0,0921 & 0,0917 & 509 \\
\hline Münchener Rück & 0,0814 & 0,0774 & 510 & 0,0910 & 0,0895 & 509 \\
\hline Fresenius Medical Care & 0,0916 & 0,0828 & 508 & 0,1106 & 0,1038 & 507 \\
\hline Adidas & 0,0929 & 0,0881 & 509 & 0,1169 & 0,1114 & 509 \\
\hline Linde & 0,0944 & 0,0870 & 510 & 0,1094 & 0,1040 & 509 \\
\hline ThyssenKrupp & 0,0945 & 0,0826 & 510 & 0,1136 & 0,1041 & 509 \\
\hline Deutsche Börse & 0,0953 & 0,0866 & 509 & 0,1182 & 0,1058 & 509 \\
\hline Merck & 0,0956 & 0,0892 & 509 & 0,1199 & 0,1143 & 509 \\
\hline Henkel & 0,0983 & 0,0909 & 510 & 0,1194 & 0,1151 & 509 \\
\hline Deutsche Post & 0,0989 & 0,0946 & 509 & 0,1201 & 0,1149 & 509 \\
\hline Beiersdorf & 0,0993 & 0,0949 & 509 & 0,1237 & 0,1181 & 509 \\
\hline Metro & 0,1000 & 0,0891 & 509 & 0,1215 & 0,1131 & 508 \\
\hline $\mathrm{K}+\mathrm{S}$ & 0,1009 & 0,0930 & 509 & 0,1263 & 0,1191 & 509 \\
\hline Volkswagen Vz. & 0,1009 & 0,1007 & 509 & 0,1246 & 0,1220 & 509 \\
\hline MAN & 0,1030 & 0,0919 & 509 & 0,1284 & 0,1147 & 509 \\
\hline Infinion & 0,1057 & 0,1010 & 508 & 0,1353 & 0,1304 & 508 \\
\hline Fresenius & 0,1088 & 0,1077 & 509 & 0,1363 & 0,1343 & 509 \\
\hline Lufthansa & 0,1155 & 0,1087 & 509 & 0,1428 & 0,1347 & 509 \\
\hline Salzgitter & 0,1196 & 0,1188 & 118 & 0,1478 & 0,1483 & 118 \\
\hline Commerzbank & 0,1288 & 0,1194 & 509 & 0,1687 & 0,1634 & 509 \\
\hline HeidelbergCement & 0,1310 & 0,1171 & 392 & 0,1606 & 0,1576 & 391 \\
\hline
\end{tabular}

Diese Tabelle zeigt den Mittelwert, den Median sowie die Anzahl der täglichen Beobachtungen (N) des $X L M_{A}$ Illiquiditätsmaßes für ein Volumen von 50.000 Euro und 100.000 Euro für die unterschiedlichen Aktien. Der Beobachtungszeitraum ist vom 01.01.2010 bis zum 31.12.2011.

Volumen von 50.000 Euro und Commerzbank mit einem $X L M_{A}$ von 0,1687\% für ein Volumen von 100.000 Euro. Für beide Volumina sind die Unterschiede zwischen Mittelwert und Median gering. Wie die Anzahl der Beobachtungen $(N)$ zeigt, ist das $X L M_{A}$ nicht zu jedem Zeitpunkt verfügbar. Beispielsweise fehlen für das Unternehmen E.ON im Beobachtungszeitraum, welcher aus 521 Tagen besteht, 13 bzw. 14 Werte.

Deskriptive Statistiken für das Liquiditätsmaß $X L M_{F}$ für kurzfristige SSF sind in Ta- 
Tabelle 4.12: Illiquiditätsmaße der kurzfristigen SSF

\begin{tabular}{|c|c|c|c|c|c|c|}
\hline & \multicolumn{6}{|c|}{ Illiquiditätsmaß kurzfristige $\mathrm{SSF}$ ( $X L M_{F}$ in Prozent) } \\
\hline & \multicolumn{3}{|c|}{ 50.000 Euro } & \multicolumn{3}{|c|}{ 100.000 Euro } \\
\hline & Mittelwert & Median & $\mathrm{N}$ & Mittelwert & Median & $\mathrm{N}$ \\
\hline Adidas & - & - & - & - & - & - \\
\hline $\mathrm{K}+\mathrm{S}$ & 0,2751 & 0,2825 & 13 & - & - & - \\
\hline Henkel & 0,2798 & 0,2321 & 29 & - & - & - \\
\hline Salzgitter & 0,2958 & 0,2977 & 8 & - & - & - \\
\hline Volkswagen Vz. & 0,4430 & 0,3748 & 307 & 0,4437 & 0,3710 & 294 \\
\hline RWE & 0,5285 & 0,3503 & 505 & 0,5781 & 0,3707 & 504 \\
\hline Siemens & 0,6068 & 0,3598 & 505 & 0,6402 & 0,3834 & 504 \\
\hline E.ON & 0,6081 & 0,4045 & 421 & 0,7671 & 0,4856 & 342 \\
\hline BASF & 0,6260 & 0,3819 & 479 & 0,6456 & 0,4047 & 440 \\
\hline Merck & 0,6345 & 0,6708 & 233 & 0,7539 & 0,9402 & 203 \\
\hline Deutsche Börse & 0,6368 & 0,3393 & 308 & 0,7903 & 0,8004 & 166 \\
\hline Daimler & 0,6515 & 0,3898 & 505 & 0,7384 & 0,5463 & 505 \\
\hline Allianz & 0,6530 & 0,3723 & 187 & 0,7016 & 0,4009 & 172 \\
\hline Beiersdorf & 0,6735 & 0,4156 & 257 & 0,9379 & 0,9535 & 67 \\
\hline Bayer & 0,6818 & 0,4342 & 505 & 0,7380 & 0,4898 & 504 \\
\hline SAP & 0,6826 & 0,5190 & 165 & 0,5125 & 0,2991 & 136 \\
\hline MAN & 0,7393 & 0,5494 & 373 & 0,9513 & 0,9766 & 360 \\
\hline BMW & 0,7579 & 0,4532 & 506 & 1,0672 & 0,9998 & 505 \\
\hline Linde & 0,8481 & 0,7589 & 502 & 1,0402 & 1,0172 & 501 \\
\hline Deutsche Bank & 0,8703 & 0,6183 & 313 & 0,9313 & 0,7074 & 312 \\
\hline Münchener Rück & 0,9605 & 0,5186 & 182 & 0,5682 & 0,2994 & 156 \\
\hline Fresenius & 1,1297 & 0,8323 & 228 & 1,3140 & 1,2450 & 205 \\
\hline Deutsche Telekom & 1,1753 & 0,7496 & 499 & 0,7035 & 0,4684 & 325 \\
\hline Lufthansa & 1,2139 & 1,1032 & 470 & 1,7861 & 1,4413 & 188 \\
\hline ThyssenKrupp & 1,2882 & 1,1952 & 502 & 1,5509 & 1,3660 & 472 \\
\hline Metro & 1,3103 & 1,2392 & 494 & 1,4179 & 1,2960 & 493 \\
\hline Fresenius Medical Care & 1,4059 & 1,3322 & 499 & 1,5183 & 1,4186 & 498 \\
\hline Deutsche Post & 1,4296 & 1,1693 & 500 & 1,7376 & 1,6595 & 180 \\
\hline HeidelbergCement & 1,4575 & 1,3815 & 383 & 1,5783 & 1,4614 & 382 \\
\hline Infinion & 1,5849 & 1,5517 & 256 & 1,6483 & 1,5383 & 69 \\
\hline Commerzbank & 2,6813 & 1,9359 & 212 & 1,9966 & 1,0649 & 84 \\
\hline
\end{tabular}

Diese Tabelle zeigt den Mittelwert, den Median sowie die Anzahl der täglichen Beobachtungen (N) des $X L M_{F}$ Illiquiditätsmaßes für ein Volumen von 50.000 Euro und 100.000 Euro für die kurzfristigen SSF. Der Beobachtungszeitraum ist vom 01.01.2010 bis zum 31.12.2011.

belle 4.12 und für langfristige SSF in Tabelle 4.13 dargestellt. Erneut werden SSF im Verfallsmonat von der Analyse ausgeschlossen. Die durchschnittliche Restlaufzeit der SSF mit der kürzesten Laufzeit beträgt ca. 34 Tage und die mit der zweitkürzesten Laufzeit ca. 65,5 Tage. Von den gesamten Beobachtungen entfallen ca. 54\% auf kurzfristige und ca. $46 \%$ auf langfristige SSF.

Tabelle 4.12 zeigt, dass die kurzfristigen SSF wesentlich illiquider als die entsprechenden 
Aktien sind. Der Mittelwert des $X L M_{F}$ für ein Volumen von 50.000 Euro (100.000 Euro) reicht für die kurzfristigen $\mathrm{SSF}$ von $0,28 \%$ bis $2,68 \%(0,44 \%$ bis $1,99 \%)$. Das Zustandekommen des kleineren $X L M_{F}$ Mittelwertes für das Volumen von 100.000 Euro kann durch die Berechnung des XLM erklärt werden. Das XLM ist ein bedingtes Maß in dem Sinne, dass es für ein gegebenes Volumen nur dann berechnet werden kann, sofern im Limit-Orderbuch Aufträge in Höhe dieses Volumens vorhanden sind. Werden in einer illiquiden Marktphase lediglich Aufträge mit einem Volumen von bis zu 50.000 Euro eingestellt, kann das XLM für ein Volumen von 100.000 Euro an diesen Tagen nicht berechnet werden. Aus der Anzahl der Beobachtungen wird deutlich, dass die fehlenden Beobachtungen mit steigendem Volumen ebenfalls zunehmen. Aufgrund dieser fehlenden Werte kann der Mittelwert des $X L M_{F}$ über den gesamten Beobachtungszeitraum für ein Volumen von 100.000 Euro geringer als der für ein Volumen von 50.000 Euro ausfallen. Die Unterschiede zwischen Mittelwert und Median sind für das $X L M_{F}$ größer als für das $X L M_{A}$ und für die meisten SSF ist der Median kleiner als der Mittelwert, was auf eine rechtsschiefe Verteilung der $X L M_{F}$ Werte schließen lässt. Die Anzahl der fehlenden Beobachtungen ist für beide Volumina größer als die am Aktienmarkt.

Ein wesentlicher Unterschied zwischen den kurz- und langfristigen SSF, der aus den Tabellen 4.12 und 4.13 ersichtlich wird, liegt in der Höhe der Illiquidität. Für ein Volumen von 50.000 Euro (100.000 Euro) liegen die mittleren $X L M_{F}$ der unterschiedlichen SSF mit Werten zwischen 0,54\% und 3,26\% (0,34\% und 4,54\%) deutlich über denen der kurzfristigen SSF. Ebenfalls Ausgeprägter für die langfristigen SSF sind die Unterschiede zwischen Mittelwert und Median sowie die Anzahl der fehlenden Beobachtungen.

Tabelle 4.14 zeigt die deskriptive Statistik zu den modelltheoretischen Variablen Volatilität und LiqRisiko im Zeitraum von Januar 2010 bis Dezember 2011. Die Volatilität der Aktienrenditen deutet mit Mittelwerten zwischen 17,11\% und 36,16\% p. a. wie im Fall der Preishypothesen auf eine Marktphase ohne extreme Schwankungen hin. Das Illiquiditätsrisiko, berechnet aus dem $X L M_{A}$ für 50.000 und 100.000 Euro, weist anders als die Volatilität extreme Ausreißer auf. Das mittlere Liquiditätsrisiko für beide Volumina liegt für etwa zwei Drittel der Aktien zwischen $0,1 \%$ und $0,7 \%$ p. a. Die übrigen Mittelwerte weisen extrem hohe Werte von bis zu 33,61\% auf. Die Unterschiede im Median der Liquiditätsrisiken sind verglichen mit den Mittelwerten gering. Folglich sind die hohen Mittelwerte das Resultat von extremen Ausreißern. Diese extremen Werte können als Liquiditätsschocks interpretiert werden. In der empirischen Analyse ist daher zu un- 
Tabelle 4.13: Illiquiditätsmaße der langfristigen SSF

\begin{tabular}{|c|c|c|c|c|c|c|}
\hline & \multicolumn{6}{|c|}{ Illiquiditätsmaß langfristige $\mathrm{SSF}\left(X L M_{F}\right.$ in Prozent) } \\
\hline & \multicolumn{3}{|c|}{ 50.000 Euro } & \multicolumn{3}{|c|}{ 100.000 Euro } \\
\hline & Mittelwert & Median & $\mathrm{N}$ & Mittelwert & Median & $\mathrm{N}$ \\
\hline Adidas & - & - & - & - & - & - \\
\hline $\mathrm{K}+\mathrm{S}$ & - & - & - & - & - & - \\
\hline Salzgitter & - & - & - & - & - & - \\
\hline Volkswagen Vz. & 0,5473 & 0,3814 & 269 & 0,5399 & 0,3771 & 261 \\
\hline Merck & 0,6616 & 0,6631 & 97 & 0,8174 & 0,9830 & 79 \\
\hline Deutsche Börse & 0,6931 & 0,3856 & 242 & 1,0625 & 0,9332 & 84 \\
\hline RWE & 0,7420 & 0,4853 & 486 & 0,7860 & 0,5978 & 478 \\
\hline Henkel & 0,7445 & 0,7445 & 2 & - & - & - \\
\hline Münchener Rück & 0,7764 & 0,6076 & 179 & 0,6149 & 0,4669 & 137 \\
\hline E.ON & 0,8121 & 0,6930 & 380 & 0,8413 & 0,5672 & 318 \\
\hline SAP & 0,8194 & 1,0342 & 147 & 0,3432 & 0,2991 & 68 \\
\hline BASF & 0,8236 & 0,7608 & 420 & 0,8353 & 0,7919 & 388 \\
\hline Siemens & 0,8757 & 0,5017 & 501 & 0,7929 & 0,5232 & 483 \\
\hline BMW & 0,8758 & 1,0717 & 459 & 1,0769 & 1,1109 & 439 \\
\hline Allianz & 0,8830 & 0,5542 & 172 & 0,9240 & 0,6573 & 161 \\
\hline Beiersdorf & 0,9248 & 0,6369 & 226 & 0,9764 & 1,0000 & 32 \\
\hline Daimler & 0,9290 & 0,7006 & 466 & 1,0314 & 1,0507 & 461 \\
\hline Bayer & 0,9534 & 1,0280 & 467 & 0,9721 & 1,0650 & 452 \\
\hline Fresenius & 1,0121 & 0,7902 & 118 & 1,3040 & 1,1753 & 88 \\
\hline MAN & 1,0393 & 1,1529 & 314 & 1,1651 & 1,1668 & 289 \\
\hline Linde & 1,1714 & 1,2644 & 448 & 1,2944 & 1,3025 & 431 \\
\hline Lufthansa & 1,1724 & 1,1137 & 388 & 1,7166 & 1,3973 & 104 \\
\hline Deutsche Post & 1,2561 & 1,1371 & 426 & 1,5651 & 1,3435 & 62 \\
\hline Metro & 1,3846 & 1,3371 & 425 & 1,4671 & 1,3749 & 416 \\
\hline ThyssenKrupp & 1,4227 & 1,2606 & 435 & 1,6028 & 1,3500 & 389 \\
\hline Deutsche Telekom & 1,4572 & 0,7867 & 426 & 0,6366 & 0,4496 & 275 \\
\hline Fresenius Medical Care & 1,5032 & 1,4645 & 423 & 1,5633 & 1,5250 & 422 \\
\hline HeidelbergCement & 1,5578 & 1,4627 & 344 & 1,6131 & 1,5116 & 342 \\
\hline Deutsche Bank & 1,5607 & 1,2656 & 304 & 1,5942 & 1,2723 & 304 \\
\hline Infinion & 1,6294 & 1,4603 & 140 & 1,7448 & 1,7746 & 22 \\
\hline Commerzbank & 3,2669 & 2,0715 & 128 & 4,5423 & 4,4007 & 22 \\
\hline
\end{tabular}

Diese Tabelle zeigt den Mittelwert, den Median sowie die Anzahl der täglichen Beobachtungen $(\mathrm{N})$ des $X L M_{F}$ Illiquiditätsmaßes für ein Volumen von 50.000 Euro und 100.000 Euro für die langfristigen SSF. Der Beobachtungszeitraum ist vom 01.01.2010 bis zum 31.12.2011.

tersuchen, ob die Ergebnisse durch diese Liquiditätsschocks getrieben bzw. beeinflusst werden.

Deskriptive Statistiken zur Geld-Brief-Spanne als alternativem Liquiditätsmaß sowie dem daraus berechneten Liquiditätsrisiko sind in Tabelle 4.15 abgebildet. Die Mittelwerte der Geld-Brief-Spanne am Aktienmarkt liegen zwischen 0,0464\% und 0,2082\% und sind damit etwas keiner als das $X M L_{A}$ für 50.000 Euro. Die Siemens AG und 
Tabelle 4.14: Volatilität und Liquiditätsrisiko der Aktien

\begin{tabular}{|c|c|c|c|c|c|c|}
\hline & \multirow{2}{*}{\multicolumn{2}{|c|}{ Volatilität (in Prozent) }} & \multicolumn{4}{|c|}{ Liquiditätsrisiko (in Prozent) } \\
\hline & & & \multicolumn{2}{|c|}{50000 Euro } & \multicolumn{2}{|c|}{100000 Euro } \\
\hline & Mittelwert & Median & Mittelwert & Median & Mittelwert & Median \\
\hline Münchener Rück & 17,11 & 21,93 & 15,1119 & 0,1126 & 14,9132 & 0,1380 \\
\hline Fresenius Medical Care & 17,84 & 19,08 & 0,4079 & 0,1596 & 0,1973 & 0,1812 \\
\hline Beiersdorf & 18,46 & 19,23 & 0,1878 & 0,1654 & 0,2312 & 0,1960 \\
\hline Deutsche Telekom & 18,54 & 21,43 & 0,1295 & 0,1330 & 0,1441 & 0,1418 \\
\hline Linde & 19,71 & 21,91 & 13,2837 & 0,1585 & 12,4046 & 0,1694 \\
\hline SAP & 19,88 & 21,35 & 0,4084 & 0,1055 & 0,1403 & 0,1215 \\
\hline RWE & 20,97 & 26,34 & 8,1943 & 0,1177 & 9,0716 & 0,1379 \\
\hline Merck & 21,02 & 23,47 & 9,2391 & 0,1647 & 9,7503 & 0,1924 \\
\hline Allianz & 21,58 & 28,55 & 13,2804 & 0,1398 & 13,3153 & 0,1548 \\
\hline Deutsche Post & 21,72 & 24,06 & 0,1818 & 0,1623 & 0,2160 & 0,1945 \\
\hline E.ON & 21,79 & 26,37 & 0,6963 & 0,1006 & 0,4201 & 0,1231 \\
\hline Bayer & 22,65 & 26,26 & 0,5226 & 0,1118 & 0,1520 & 0,1314 \\
\hline Henkel & 22,83 & 24,30 & 0,4631 & 0,1813 & 0,2331 & 0,2083 \\
\hline Fresenius & 22,84 & 23,01 & 0,1986 & 0,1673 & 0,2697 & 0,2070 \\
\hline Deutsche Börse & 24,22 & 28,39 & 5,2268 & 0,1843 & 5,5112 & 0,2212 \\
\hline Siemens & 24,23 & 26,38 & 9,9121 & 0,0868 & 9,6329 & 0,1024 \\
\hline Adidas & 24,96 & 27,44 & 0,1957 & 0,1625 & 0,2395 & 0,1982 \\
\hline Metro & 25,03 & 29,04 & 0,5205 & 0,1748 & 0,2457 & 0,2133 \\
\hline BASF & 25,97 & 28,42 & 0,4440 & 0,1144 & 0,1423 & 0,1303 \\
\hline $\mathrm{K}+\mathrm{S}$ & 26,74 & 30,41 & 0,2257 & 0,1861 & 0,2797 & 0,2296 \\
\hline Lufthansa & 26,86 & 30,04 & 0,2274 & 0,2057 & 0,2706 & 0,2498 \\
\hline Daimler & 28,34 & 32,74 & 3,3842 & 0,1233 & 3,7480 & 0,1486 \\
\hline ThyssenKrupp & 28,59 & 33,98 & 0,5229 & 0,1575 & 0,2177 & 0,1891 \\
\hline Deutsche Bank & 28,62 & 36,68 & 7,9891 & 0,1236 & 8,0458 & 0,1512 \\
\hline BMW & 28,76 & 33,16 & 2,0649 & 0,1248 & 1,8749 & 0,1473 \\
\hline Salzgitter & 29,27 & 34,16 & 25,0526 & 0,2470 & 33,6163 & 0,2888 \\
\hline MAN & 29,31 & 33,07 & 8,2008 & 0,2003 & 8,3931 & 0,2390 \\
\hline Volkswagen Vz. & 33,92 & 36,58 & 0,2212 & 0,1861 & 0,2796 & 0,2323 \\
\hline Infinion & 35,09 & 39,78 & 0,2423 & 0,2035 & 0,3045 & 0,2552 \\
\hline Commerzbank & 35,79 & 42,03 & 0,2851 & 0,2661 & 0,3885 & 0,3770 \\
\hline HeidelbergCement & 36,16 & 38,15 & 0,8162 & 0,2189 & 0,3288 & 0,2888 \\
\hline
\end{tabular}

Diese Tabelle zeigt den Mittelwert, den Median sowie die Anzahl der täglichen Beobachtungen (N) der Volatilität (Volatilität) und des Liquiditätsrisikos (LiqRisiko) der Aktien. Der Beobachtungszeitraum ist vom 01.01.2010 bis zum 31.12.2011.

die Commerzbank AG stellen auch gemessen durch die Geld-Brief-Spanne die liquideste bzw. illiquideste Aktie dar. Zwischen Mittelwert und Median gibt es für keine Aktie große Abweichungen. Die Variable LiqRisiko $_{G B S}$ liegt zwischen 0,2120\% und 1,5660\% p. a. Anders als bei der Variable LiqRisiko sind die Unterschiede zwischen Mittelwert und Median der Variable LiqRisiko ${ }_{G B S}$ klein.

In Tabelle 4.16 wird die deskriptive Statistik zur Geld-Brief-Spanne der SSF darge- 
Tabelle 4.15: Geld-Brief-Spanne und Liquiditätsrisiko der Aktien

\begin{tabular}{|c|c|c|c|c|c|c|}
\hline & \multicolumn{3}{|c|}{$G B S_{A}$ (in Prozent) } & \multicolumn{3}{|c|}{ Liquiditätsrisiko (in Prozent) } \\
\hline & Mittelwert & Median & $\mathrm{N}$ & Mittelwert & Median & $\mathrm{N}$ \\
\hline Siemens & 0,0464 & 0,0415 & 497 & 0,3815 & 0,3187 & 497 \\
\hline SAP & 0,0517 & 0,0477 & 497 & 0,3633 & 0,3037 & 497 \\
\hline RWE & 0,0519 & 0,0437 & 497 & 0,3837 & 0,3222 & 497 \\
\hline BASF & 0,0560 & 0,0495 & 497 & 0,4310 & 0,4104 & 497 \\
\hline Allianz & 0,0572 & 0,0491 & 497 & 0,4181 & 0,3797 & 497 \\
\hline E.ON & 0,0594 & 0,0459 & 497 & 0,3616 & 0,2761 & 497 \\
\hline Deutsche Bank & 0,0594 & 0,0490 & 497 & 0,4807 & 0,3251 & 497 \\
\hline Daimler & 0,0599 & 0,0561 & 497 & 0,4543 & 0,4195 & 497 \\
\hline Bayer & 0,0605 & 0,0522 & 497 & 0,5465 & 0,3790 & 497 \\
\hline BMW & 0,0736 & 0,0659 & 497 & 0,6126 & 0,5675 & 497 \\
\hline Fresenius Medical Care & 0,0738 & 0,0647 & 497 & 0,6025 & 0,5059 & 497 \\
\hline ThyssenKrupp & 0,0745 & 0,0679 & 497 & 0,5493 & 0,4774 & 497 \\
\hline Münchener Rück & 0,0771 & 0,0805 & 497 & 0,5190 & 0,4544 & 497 \\
\hline Henkel & 0,0794 & 0,0702 & 497 & 0,6713 & 0,6632 & 497 \\
\hline Linde & 0,0805 & 0,0818 & 497 & 0,5849 & 0,5515 & 497 \\
\hline Merck & 0,0819 & 0,0668 & 497 & 0,7922 & 0,6364 & 497 \\
\hline Metro & 0,0840 & 0,0697 & 497 & 0,7566 & 0,6186 & 497 \\
\hline Adidas & 0,0847 & 0,0703 & 497 & 0,8392 & 0,7108 & 497 \\
\hline $\mathrm{K}+\mathrm{S}$ & 0,0884 & 0,0741 & 497 & 0,7131 & 0,6079 & 497 \\
\hline MAN & 0,0908 & 0,0724 & 497 & 0,8331 & 0,7053 & 497 \\
\hline Volkswagen Vz. & 0,0908 & 0,0820 & 497 & 0,8171 & 0,7546 & 497 \\
\hline Beiersdorf & 0,0913 & 0,0731 & 497 & 0,8390 & 0,8311 & 497 \\
\hline Deutsche Post & 0,1030 & 0,0796 & 497 & 0,6230 & 0,5607 & 497 \\
\hline Deutsche Telekom & 0,1081 & 0,0999 & 497 & 0,2120 & 0,0509 & 497 \\
\hline HeidelbergCement & 0,1112 & 0,0876 & 379 & 1,2195 & 1,2765 & 379 \\
\hline Salzgitter & 0,1116 & 0,1027 & 118 & 0,8193 & 0,8512 & 118 \\
\hline Lufthansa & 0,1120 & 0,0889 & 497 & 0,7470 & 0,6532 & 497 \\
\hline Deutsche Börse & 0,1242 & 0,0752 & 497 & 1,1151 & 0,5948 & 497 \\
\hline Fresenius & 0,1570 & 0,1157 & 497 & 1,5660 & 1,7001 & 497 \\
\hline Infinion & 0,1802 & 0,1704 & 497 & 0,4156 & 0,1989 & 497 \\
\hline Commerzbank & 0,2082 & 0,1688 & 497 & 0,4404 & 0,3655 & 497 \\
\hline
\end{tabular}

Diese Tabelle zeigt den Mittelwert, den Median sowie die Anzahl der täglichen Beobachtungen (N) der Geld-Brief-Spanne $\left(G B S_{A}\right)$ und des Liquiditätsrisikos $\left(\right.$ LiqRisiko $\left._{G B S}\right)$ der Aktien. Der Beobachtungszeitraum ist vom 01.01.2010 bis zum 31.12.2011.

stellt. Es entfallen ca. $52 \%$ (48\%) der Beobachtung auf kurzfristige (langfristige) SSFKontrakte mit einer durchschnittlichen Restlaufzeit von ca. 31 Tagen (62 Tagen). Die Illiquidität ist mit einer Geld-Brief-Spanne von $0,2815 \%$ bis 2,7868\% (0,3045\% bis $2,6106 \%$ ) für kurzfristige (langfristige) SSF-Kontrakte deutlich höher als die Geld-BriefSpanne am Aktienmarkt. Auch gemessen durch die Geld-Brief-Spanne sind die Mittelwerte der kurzfristigen SSF überwiegend kleiner als die der langfristigen SSF. Auffällig ist, dass die Illiquidität der SSF gemessen durch die Geld-Brief-Spanne größer ist, als 
Tabelle 4.16: Geld-Brief-Spanne der SSF

\begin{tabular}{|c|c|c|c|c|c|c|}
\hline & \multicolumn{6}{|c|}{ Geld-Brief-Spanne $\left(G B S_{F}\right.$ in Prozent) } \\
\hline & \multicolumn{3}{|c|}{ kurzfristige SSF } & \multicolumn{3}{|c|}{ langfristige SSF } \\
\hline & Mittelwert & Median & $\mathrm{N}$ & Mittelwert & Median & $\mathrm{N}$ \\
\hline $\mathrm{K}+\mathrm{S}$ & 0,2815 & 0,2804 & 228 & 0,3045 & 0,2804 & 201 \\
\hline Henkel & 0,2992 & 0,2991 & 3 & 0,3988 & 0,3989 & 3 \\
\hline Salzgitter & 0,3273 & 0,3151 & 18 & 0,5991 & 0,5991 & 2 \\
\hline Volkswagen Vz. & 0,3689 & 0,2811 & 300 & 0,5121 & 0,2812 & 274 \\
\hline Merck & 0,4516 & 0,2816 & 353 & 0,4964 & 0,2810 & 243 \\
\hline Beiersdorf & 0,5200 & 0,2997 & 447 & 0,6987 & 0,4140 & 365 \\
\hline Deutsche Börse & 0,6537 & 0,2822 & 346 & 0,6691 & 0,3432 & 267 \\
\hline Fresenius & 0,8300 & 0,2812 & 337 & 0,7338 & 0,2807 & 259 \\
\hline MAN & 0,9432 & 1,1327 & 379 & 1,1279 & 1,1744 & 332 \\
\hline Deutsche Post & 1,0781 & 1,0913 & 497 & 1,1781 & 1,1099 & 445 \\
\hline RWE & 1,1582 & 1,1119 & 497 & 1,1992 & 1,1399 & 478 \\
\hline Deutsche Telekom & 1,2105 & 1,0347 & 497 & 1,4356 & 1,0946 & 493 \\
\hline Allianz & 1,2223 & 1,0515 & 497 & 1,2367 & 1,0565 & 497 \\
\hline Lufthansa & 1,2541 & 1,1317 & 495 & 1,3778 & 1,1731 & 442 \\
\hline E.ON & 1,2728 & 0,5794 & 482 & 1,3942 & 1,0544 & 477 \\
\hline Siemens & 1,2955 & 1,0938 & 497 & 1,4597 & 1,1093 & 493 \\
\hline ThyssenKrupp & 1,2961 & 1,1652 & 497 & 1,3417 & 1,1764 & 486 \\
\hline Fresenius Medical Care & 1,3268 & 1,3586 & 494 & 1,4763 & 1,4904 & 445 \\
\hline Linde & 1,3304 & 1,3778 & 497 & 1,4801 & 1,4246 & 445 \\
\hline Adidas & 1,3348 & 1,2908 & 496 & 1,4405 & 1,3618 & 445 \\
\hline SAP & 1,3643 & 1,0449 & 497 & 1,3763 & 1,0550 & 497 \\
\hline BASF & 1,3970 & 1,0749 & 483 & 1,5655 & 1,0915 & 443 \\
\hline Daimler & 1,4282 & 1,1073 & 497 & 1,7908 & 1,1399 & 459 \\
\hline HeidelbergCement & 1,4706 & 1,4552 & 378 & 1,6477 & 1,5064 & 364 \\
\hline BMW & 1,5170 & 1,1353 & 497 & 1,5433 & 1,1585 & 451 \\
\hline Bayer & 1,5371 & 1,1451 & 497 & 1,7643 & 1,1879 & 469 \\
\hline Deutsche Bank & 1,5633 & 1,1112 & 497 & 1,7895 & 1,1836 & 485 \\
\hline Metro & 1,7036 & 1,2933 & 494 & 1,8750 & 1,3509 & 443 \\
\hline Infinion & 1,8513 & 1,1672 & 497 & 1,8714 & 1,1659 & 497 \\
\hline Commerzbank & 2,0046 & 1,1756 & 404 & 2,0470 & 1,2533 & 357 \\
\hline Münchener Rück & 2,7868 & 1,4169 & 191 & 2,6106 & 1,2542 & 191 \\
\hline
\end{tabular}

Diese Tabelle zeigt den Mittelwert, den Median sowie die Anzahl der täglichen Beobachtungen (N) der Geld-Brief-Spanne $\left(G B S_{F}\right)$ der kurz- und langfristigen SSF. Der Beobachtungszeitraum ist vom 01.01.2010 bis zum 31.12.2011.

wenn diese durch das $X L M_{F}$ für ein Volumen von 50.000 Euro gemessen wird. Dieser Vergleich ist jedoch aus zwei Gründen problematisch: Erstens ist das XLM ein Tagesdurchschnitt und die Geld-Brief-Spanne bezieht sich auf den Zeitpunkt um 16.00 Uhr. Zweitens kann das XLM nur berechnet werden, sofern ausreichend Liquidität zur Verfügung steht. Auch an Tagen an denen aufgrund eines leeren Orderbuchs kein $X L M$ berechnet werden kann, sind die Market Maker verpflichtet Geld- und Briefkurse zu 
stellen. Folglich gehen die besonders illiquiden Tage nicht bei der Berechnung des $X L M$ ein. Für die Geld-Brief-Spanne sind diese Tage jedoch verfügbar, was die Verzerrung des $X L M$ bzw. die großen Werte der Geld-Brief-Spanne erklärt.

\subsubsection{Determinanten der Futuresliquidität}

Der Fokus dieses Abschnitts liegt auf der Analyse der modelltheoretischen Hypothesen zur Liquiditätsübertragung. Die Robustheit der Ergebnisse wird gewährleistet, indem unterschiedliche Maße für die modelltheoretischen Variablen untersucht werden und für weitere nicht im Modell enthaltene Friktionen kontrolliert wird. Im letzten Teil dieses Abschnitts werden Indexfuturesdaten anstelle der SSF-Daten verwendet. Auf diese Weise können die modelltheoretischen Vorhersagen anhand eines Datensatzes überprüft werden, für den das Substitutionsargument eine größere Bedeutung haben sollte.

\subsubsection{Basisfall}

Zur Überprüfung der Liquiditätshypothesen dienen ebenfalls Fixed-Effects Panelregressionen. Diese haben die Form:

$$
X L M_{F i, t}=\alpha_{i}+\beta x_{i, t}+\varepsilon_{i, t},
$$

mit $X L M_{F i, t}$ als Illiquiditätsmaß des SSF auf die Aktie $i$ zum Zeitpunkt $t$. Es handelt sich wie bei der Überprüfung der Preishypothesen um einen unbalancierten Paneldatensatz und es wird weiterhin angenommen, dass der Fehlerterm $\varepsilon_{i, t}$ einen Mittelwert von null hat und unabhängig von dem Vektor der erklärenden Variablen $x_{i, t}$ ist. Die in Abschnitt 4.3.2 eingeführten erklärenden Variablen, die dem Modell nach die Illiquidität der SSF beeinflussen sollen, sind gegeben als $X L M_{S}$, Volatilität, LiqRisiko und Laufzeit. Eine Panelregression mit diesen Variablen bildet daher den Basisfall zur Überprüfung der Liquiditätshypothesen.

Die Ergebnisse der Regression sind in Tabelle 4.17 abgebildet. Die verwendeten XLM Maße beziehen sich auf ein Volumen von 50.000 und 100.000 Euro. Die p-Werte sind erneut in Klammern unter den Schätzern für die Parameterwerte angegeben und basieren auf geclusterten Standardfehlern nach Petersen (2009). Sämtliche Ergebnisse unterstützen die modelltheoretischen Vorhersagen. Für eine Auftragsgröße von 50.000 Euro sind 
Tabelle 4.17: Determinanten der Futuresliquidität: Basisfall.

\begin{tabular}{|c|c|c|c|c|}
\hline & \multicolumn{2}{|c|}{$X L M_{F}$} & & \multirow{2}{*}{$G B S_{F}$} \\
\hline & 50.000 Euros & 100.000 Euros & & \\
\hline$X L M_{A}$ & $\begin{array}{l}0,223^{* * *} \\
(0,000)\end{array}$ & $\begin{array}{l}0,936^{* *} \\
(0,016)\end{array}$ & $G B S_{A}$ & $\begin{array}{l}0,841^{* * *} \\
(0,001)\end{array}$ \\
\hline Volatilität & $\begin{array}{l}0,006^{* * *} \\
(0,002)\end{array}$ & $\begin{array}{l}0,004^{* *} \\
(0,036)\end{array}$ & Volatilität & $\begin{array}{l}0,025^{* * *} \\
(0,000)\end{array}$ \\
\hline LiqRisiko & $\begin{array}{l}0,002^{* * *} \\
(0,000)\end{array}$ & $\begin{array}{l}0,002^{* * *} \\
(0,000)\end{array}$ & LiqRisiko $_{G B S}$ & $\begin{array}{l}-0,005 \\
(0,956)\end{array}$ \\
\hline Laufzeit & $\begin{array}{l}0,173^{* * *} \\
(0,000)\end{array}$ & $\begin{array}{l}0,149^{* * *} \\
(0,000)\end{array}$ & Laufzeit & $\begin{array}{l}0,121^{* * *} \\
(0,000)\end{array}$ \\
\hline Konstante & $\begin{array}{l}0,729^{* * *} \\
(0,000)\end{array}$ & $\begin{array}{l}0,755^{* * *} \\
(0,000)\end{array}$ & Konstante & $\begin{array}{l}0,503^{\text {*** }} \\
(0,000)\end{array}$ \\
\hline $\begin{array}{l}\text { Beobachtungen } \\
\text { within }-R^{2}\end{array}$ & $\begin{array}{l}18.886 \\
0,0216\end{array}$ & $\begin{array}{l}15.189 \\
0,0627\end{array}$ & & $\begin{array}{l}21.988 \\
0,0480\end{array}$ \\
\hline
\end{tabular}

Die Tabelle zeigt die Ergebnisse der Fixed-Effects Panelregressionen mit der Liquidität des Futuresmarktes als abhängiger Variable. Die abhängige Variable der ersten beiden Regressionen ist das Liquiditätsmaß $X L M_{F}$ für ein Volumen von 50.000 Euro und 100.000 Euro. Die erklärenden Variablen sind XLM , Volatilität, LiqRisiko, und Laufzeit. Die abhängige Variable der dritten Regression ist die Geld-Brief-Spanne $G B S_{F}$. Die erklärenden Variablen sind $G B S_{S}$, Volatilität, LiqRisiko $_{G B S}$, und Laufzeit. Der als Konstante ausgewiesene Wert ist der mean fixed effect. Die p-Werte sind in Klammern angegeben und wurden bestimmt durch geclusterte Standardfehler. Der Beobachtungszeitraum ist vom 01.01.2010 bis zum 31.12.2011. ${ }^{* *},{ }^{* *}$, und $*$ geben die statistische Signifikanz auf dem 1\%, $5 \%$, and $10 \%$ Niveau an.

alle vier aus dem Modell abgeleiteten Determinanten auf dem $1 \%$ Niveau statistisch signifikant und besitzen das erwartete Vorzeichen. Eine steigende Illiquidität, eine höhere Volatilität und ein höheres Liquiditätsrisiko am Aktienmarkt sowie eine längere Restlaufzeit führen zu einer höheren Illiquidität der SSF-Kontrakte.

Die Ergebnisse für eine Auftragsgröße von 100.000 Euro stellen einen ersten Robustheitstest dar. Alle Koeffizienten sind weiterhin statistisch signifikant mit dem erwarteten Vorzeichen. Der geringe Anstieg der p-Werte könnte auf die geringere Anzahl an Beobachtungen für diese Auftragsgröße zurückzuführen sein.

In der letzten Spalte sind die Ergebnisse einer Regression abgebildet, in der die Illiquidität am Aktienmarkt und SSF-Markt durch die Geld-Brief-Spanne gemessen wird. Die Resultate sind vergleichbar mit den Regressionen, die das $X M L$ verwenden. Insbesondere sind die Geld-Brief-Spanne und die Volatilität am Aktienmarkt sowie eine längere Laufzeit signifikant und positiv mit der Illiquidität der SSF-Kontrakte verbunden. Die Effekte dieser Variablen können damit als robust gegenüber der Wahl des Liquiditätsmaßes angesehen werden. Die einzige Variable, für die nach dem Modell eine Abweichung zu erwarten ist, ist das Liquiditätsrisiko. Die Regressionsergebnisse zeigen, dass das Li- 
quiditätsrisiko nicht signifikant ist, wenn es durch die annualisierte Standardabweichung der Geld-Brief-Spanne am Aktienmarkt gemessen wird. Die Modellvorhersage, dass das Liquiditätsrisiko nur im Fall von großen Aufträgen einen positiven Effekt auf die Illiquidität am SSF-Markt hat, kann damit bestätigt werden.

\subsubsection{Alternative Risikomaße}

In den Basisregressionen wird sowohl das Preisrisiko als auch als auch das Illiquiditätsrisiko durch die Standardabweichung der Aktienrenditen bzw. des Aktienilliquiditätsmaßes berechnet. In diesem Abschnitt soll untersucht werden, ob die Ergebnisse robust gegenüber alternativen Risikomaßen sind.

Zunächst werden die Effekte von systematischen Risikomaßen auf die Illiquidität am SSF-Markt untersucht. Die Variable für das systematische Preisrisiko (Syst_Risiko) wird dabei wie im Abschnitt 4.2.3.2 berechnet. Zusätzlich zum systematischen Preisrisiko wird das systematische Liquiditätsrisiko als Kovarianz der täglichen Illiquidität der Einzelaktien mit Illiquidität des Aktienindex über ein rollierendes Fenster der letzten 30 Handelstage berechnet. Die Illiquidität des Aktienindex wird dabei als arithmetisches Mittel der Illiquidität der Einzelaktien berechnet. Die erste Variable (Syst_LiqRisiko) für das systematische Liquiditätsrisiko wird auf Basis des XLM und die zweite Variable (Syst_LiqRisiko $\left.{ }_{G B S}\right)$ auf Basis der Geld-Brief-Spanne berechnet.

Tabelle 4.18 Panel A zeigt die Regressionsergebnisse unter Verwendung von Maßen für systematisches Risiko. Die Ergebnisse sind mit denen aus dem Basisfall vergleichbar. Insbesondere ist der Koeffizient für das systematische Preisrisiko in allen drei Regressionen positiv und signifikant. Auch für das systematische Liquiditätsrisiko können die im Basisfall nachgewiesenen Effekte bestätigt werden. Für die Variable Syst_LiqRisiko ${ }_{X M}$ lässt sich ein positiver und signifikanter Zusammenhang zur Illiquidität am Futuresmarkt nachweisen. Für die Variable Syst_LiqRisiko $o_{G B S}$ kann dagegen wie im Basisfall kein signifikanter Zusammenhang nachgewiesen werden. Die übrigen modelltheoretischen Variablen sind weiterhin signifikant mit dem erwarteten Vorzeichen. Folglich sind die Ergebnisse des Basisfalls als robust gegenüber den Maßen für systematisches Risiko zu bezeichnen.

Anhand der deskriptiven Statistik ist zu erkennen, dass insbesondere das aus dem $X L M_{A}$ berechnete Liquiditätsrisiko von extremen Ausreißern geprägt ist. Ziel des nächsten Analyseschrittes ist es, zu untersuchen, ob die Ergebnisse der Basisregressionen weiterhin 
Tabelle 4.18: Determinanten der Futuresliquidität: Effekte alternativer Risikomaße

Panel A: Maße für systematisches Risiko

\begin{tabular}{|c|c|c|c|c|}
\hline & \multicolumn{2}{|c|}{$X M L_{F}$} & & \multirow{2}{*}{$G B S_{F}$} \\
\hline & 50,000 Euros & 100,000 Euros & & \\
\hline$X L M_{A}$ & $\begin{array}{l}0,226^{* * *} \\
(0,002)\end{array}$ & $\begin{array}{l}0,970^{* *} \\
(0,013)\end{array}$ & $G B S_{A}$ & $\begin{array}{l}0,864^{* * *} \\
(0,001)\end{array}$ \\
\hline Syst_Risiko & $\begin{array}{l}0,031^{* * *} \\
(0,001)\end{array}$ & $\begin{array}{l}0,020^{* *} \\
(0,035)\end{array}$ & Syst_Risiko & $\begin{array}{l}0,148^{* * *} \\
(0,000)\end{array}$ \\
\hline Syst_LiqRisiko & $\begin{array}{l}0,007^{* * *} \\
(0,000)\end{array}$ & $\begin{array}{l}0,006^{* *} \\
(0,001)\end{array}$ & Syst_LiqRisiko $G B S$ & $\begin{array}{r}-75,995 \\
(0,200)\end{array}$ \\
\hline Laufzeit & $\begin{array}{l}0,173^{* * *} \\
(0,000)\end{array}$ & $\begin{array}{l}0,149^{* * *} \\
(0,000)\end{array}$ & Laufzeit & $\begin{array}{l}0,120^{* * *} \\
(0,000)\end{array}$ \\
\hline Konstante & $\begin{array}{l}0,853^{* * *} \\
(0,000)\end{array}$ & $\begin{array}{l}0,834^{* * *} \\
(0,000)\end{array}$ & Konstante & $\begin{array}{l}0,945^{* * *} \\
(0,000)\end{array}$ \\
\hline $\begin{array}{l}\text { Beobachtungen } \\
\text { within }-R^{2}\end{array}$ & $\begin{array}{l}18.860 \\
0,0192\end{array}$ & $\begin{array}{l}15.189 \\
0,0640\end{array}$ & & $\begin{array}{l}21.988 \\
0,0524\end{array}$ \\
\hline \multicolumn{5}{|c|}{ Panel B: Robuste Risikomaße } \\
\hline & \multicolumn{2}{|c|}{$X M L_{F}$} & & \multirow{2}{*}{$G B S_{F}$} \\
\hline & 50,000 Euros & 100,000 Euros & & \\
\hline$X L M_{A}$ & $\begin{array}{l}0,228^{* * *} \\
(0,001)\end{array}$ & $\begin{array}{l}0,974^{* *} \\
(0,015)\end{array}$ & $G B S_{A}$ & $\begin{array}{l}1,056^{* * *} \\
(0,000)\end{array}$ \\
\hline$I Q A \_$Volatilität & $\begin{array}{l}0,003^{* * *} \\
(0,005)\end{array}$ & $\begin{array}{l}0,002 \\
(0,112)\end{array}$ & $I Q A \_$Volatilität & $\begin{array}{l}0,013^{* * *} \\
(0,000)\end{array}$ \\
\hline$I Q A \_$LiqRisiko & $\begin{array}{l}0,392^{* *} \\
(0,032)\end{array}$ & $\begin{array}{l}0,226^{* *} \\
(0,047)\end{array}$ & $I Q A_{\_}$LiqRisiko $G B S$ & $\begin{array}{c}0,096 \\
(0,236)\end{array}$ \\
\hline Laufzeit & $\begin{array}{l}0,173^{* * *} \\
(0,000)\end{array}$ & $\begin{array}{l}0,150^{* * *} \\
(0,000)\end{array}$ & Laufzeit & $\begin{array}{l}0,120^{* * *} \\
(0,000)\end{array}$ \\
\hline Konstante & $\begin{array}{l}0,732^{* * *} \\
(0,000)\end{array}$ & $\begin{array}{l}0,759^{* * *} \\
(0,000)\end{array}$ & Konstante & $\begin{array}{l}0,662^{* * *} \\
(0,000)\end{array}$ \\
\hline $\begin{array}{l}\text { Beobachtungen } \\
\text { within }-R^{2}\end{array}$ & $\begin{array}{l}18.860 \\
0,0399\end{array}$ & $\begin{array}{l}15.189 \\
0,0738\end{array}$ & & $\begin{array}{l}21.988 \\
0,0416\end{array}$ \\
\hline
\end{tabular}

Die Tabelle zeigt die Ergebnisse der Fixed-Effects Panelregressionen mit der Liquidität des Futuresmarktes als abhängiger Variable. Die abhängige Variable der ersten beiden Regressionen ist das Liquiditätsmaß $X L M_{F}$ für ein Volumen von 50.000 Euro und 100.000 Euro. Die erklärenden Variablen sind XLM S, Syst_Risiko (Panel A), IQA_Volatilität (Panel B), Syst_LiqRisiko (Panel A), IQA_LiqRisiko (Panel B), und Laufzeit. Die abhängige Variable der dritten Regression ist die Geld-Brief-Spanne $G B S_{F}$. Die erklärenden Variablen sind $G B S_{A}$, Syst_Risiko (Panel A), IQA_Volatilität (Panel B),

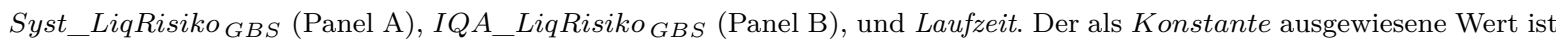
der mean fixed effect. Die p-Werte sind in Klammern angegeben und wurden bestimmt durch geclusterte Standardfehler. Der Beobachtungszeitraum ist vom 01.01.2010 bis zum 31.12.2011. ***,**, und * geben die statistische Signifikanz auf dem $1 \%, 5 \%$, and $10 \%$ Niveau an.

Bestand haben, wenn den extremen Werten bei der Berechnung der Risikomaße weniger Gewicht beigemessen wird. Zu diesem Zweck wird der Interquartilsabstand (IQA) als 
robuster Schätzer für die Volatilität der Aktienrenditen und der Aktienilliquidität verwendet. Im Unterschied zur Standardabweichung wird bei der Berechnung des IQA extremen Werten kein Gewicht beigemessen. Ökonomisch lässt sich dieses Volatilitätsmaß folglich als ein Maß interpretieren, das weniger stark auf Preis- bzw. Liquiditätsschocks reagiert.

Die Regressionsergebnisse der robusten Risikomaße sind in Panel B der Tabelle 4.18 angegeben. Die Variablen für die Aktienmarktliquidität und Laufzeit der Kontrakte sind in allen drei Spezifikationen weiterhin signifikant mit den erwarteten Vorzeichen. Die Ergebnisse für das Liquiditätsrisiko werden ebenfalls nicht durch die robuste Risikomessung beeinflusst. Wie im Basisfall ist das durch den IQA gemessene und aus dem $X L M$ berechnete Liquiditätsrisiko (IQA_LiqRisk) positiv und signifikant. Für die aus der Geld-Brief-Spanne berechnete Variable IQA_LiqRisk $k_{G B S}$ kann in Übereinstimmung zum Basisfall kein Zusammenhang zu der Illiquidität am SSF-Markt festgestellt werden. Folglich werden die Ergebnisse der Basisregression nicht durch Liquiditätsschocks getrieben. Die Variable für das robuste Preisrisiko IQA_Volatilität hat ein positives Vorzeichen und ist signifikant in den Regressionen, die das XLM für ein Volumen von 50.000 Euro und die Geld-Brief-Spanne verwenden. Lediglich in der XLM Regression für ein Volumen von 100.000 Euro liegt der Koeffizient von IQA_Volatilität knapp unter dem $10 \%$ Signifikanzniveau. Insgesamt lassen die Resultate den Schluss zu, dass die Ergebnisse robust gegenüber den alternativen Maßen für Preis- und Liquiditätsrisiko sind.

\subsubsection{Dividendeneffekte}

Tabelle 4.19 zeigt, dass SSF auch im Untersuchungszeitraum vom 01.01.2010 bis zum 31.12.2011 verstärkt um den Ex-Tag gehandelt werden. Dieses zusätzliche Handelsvolumen könnte anders als bei den Preishypothesen sowohl einen positiven als auch einen negativen Effekt auf die Illiquidität am SSF-Markt haben. Ist der Wettbewerb zwischen den Market Makern unvollkommen, wäre wie im Fall der Preishypothesen ein positiver Zusammenhang zwischen dem Ex-Tag und der Illiquidität am SSF-Markt zu erwarten. In diesem Fall würden die Market Maker die zusätzliche Nachfrage antizipieren und würden, sofern sie nicht wissen, ob es sich um eine Netto-Nachfrage nach Short- oder Longpositionen handelt, durch das Setzen einer breiteren Geld-Brief-Spanne ihren Profit erhöhen. Sind die Investoren, welche Dividendenstripping betreiben, uninformiert, wäre dagegen ein negativer Zusammenhang zu erwarten. Durch die zusätzlichen uninformier- 
Tabelle 4.19: SSF Handelsvolumen (01.01.2010 - 31.12.2011)

\begin{tabular}{lccc}
\hline & \multicolumn{3}{c}{ Mittelwert Handelsvolumen (in Stück) } \\
\cline { 2 - 4 } & vollständiger Zeitraum & 40 Tage um Ex-Tag & 10 Tage um Ex-Tag \\
\hline kurzfristige SSF & 4.155 & 19.925 & 66.705 \\
langfristige SSF & 518 & 2.276 & 7.676 \\
\hline
\end{tabular}

Diese Tabelle zeigt das mittlere Handelsvolumen der kurz- und langfristigen SSF in Stück für den vollständigen Beobachtungszeitraum, ein symmetrisches Fenster von 40 sowie 10 Tagen um den Ex-Dividendentag. Der Beobachtungszeitraum ist vom 01.01.2010 bis zum 31.12.2011.

ten Investoren um den Ex-Tag, wären die Market Maker geringeren Kosten adverser Selektion ausgesetzt, was wiederum zu einer geringeren Illiquidität führen würde.

In Tabelle 4.20 Panel A (Panel B) wird der Basisfall um die Variable Dividende 10 Tage (Dividende 40 Tage) erweitert, um so die Auswirkungen des gestiegenen Handelsvolumen um den Ex-Tag zu untersuchen. Sowohl in Panel A als auch in Panel B bleiben die modelltheoretischen Variablen unbeeinflusst von der zusätzlichen Kontrollvariable, was die Robustheit des Basisfalls und die Bedeutung der modelltheoretischen Variablen erneut bestätigt. Beide Dividenden Dummys haben in allen Regressionen ein positives Vorzeichen. Die Evidenz für einen unvollständigen Wettbewerb zwischen den Market Makern ist dennoch lediglich als schwach zu bezeichnen, da die Dividenden Dummys lediglich in den Regressionen, die die Geld-Brief-Spanne verwenden, auf dem $10 \%$ Niveau signifikant sind. In den Regressionen, die das XLM als Illiquiditätsmaß verwenden, kann dagegen keine Signifikanz nachgewiesen werden. Eine mögliche Erklärung für die insignifikanten Ergebnisse ist das gemeinsame Auftreten von uninformierten Investoren um den Ex-Tag und unvollständigem Wettbewerb zwischen den Market Makern. In diesem Fall würden sich die entgegengesetzten Effekte aufheben und dadurch nicht nachweisen lassen.

\subsubsection{Effekte von Finanzierungskosten}

In diesem Abschnitt sollen die Auswirkungen von Finanzierungskosten auf die Illiquidität am SSF-Markt untersucht werden. Aufgrund der Margin Anforderungen von SSFPositionen sind die Market Maker Kosten ausgesetzt, die von der Größe ihrer SSFPosition abhängen. Wie bereits im Rahmen der Preishypothesen erläutert, können sie durch das Verschieben des Midquotes Anreize setzen, um eine Verringerung ihrer SSFPosition und den damit verbundenen Kosten zu erreichen. Diese Anreize können nicht 
Tabelle 4.20: Determinanten der Futuresliquidität: Dividenden

Panel A: 10 Tage um Ex-Tag

\begin{tabular}{|c|c|c|c|c|}
\hline & \multicolumn{2}{|c|}{$X M L_{F}$} & & \multirow{2}{*}{$G B S_{F}$} \\
\hline & 50,000 Euros & 100,000 Euros & & \\
\hline$X L M_{A}$ & $\begin{array}{l}0,224^{* * *} \\
(0,000)\end{array}$ & $\begin{array}{l}0,940^{* *} \\
(0,016)\end{array}$ & $G B S_{A}$ & $\begin{array}{l}0,848^{* * *} \\
(0,001)\end{array}$ \\
\hline Volatilität & $\begin{array}{l}0,006^{* * *} \\
(0,002)\end{array}$ & $\begin{array}{l}0,004^{* *} \\
(0,034)\end{array}$ & Volatilität & $\begin{array}{l}0,025^{* * *} \\
(0,000)\end{array}$ \\
\hline LiqRisiko & $\begin{array}{l}0,002^{* * *} \\
(0,000)\end{array}$ & $\begin{array}{l}0,002^{* * *} \\
(0,000)\end{array}$ & LiqRisiko $_{G B S}$ & $\begin{array}{l}-0,000 \\
(0,998)\end{array}$ \\
\hline Laufzeit & $\begin{array}{l}0,173^{* * *} \\
(0,000)\end{array}$ & $\begin{array}{l}0,150 * * * \\
(0,000)\end{array}$ & Laufzeit & $\begin{array}{l}0,120 * * * \\
(0,000)\end{array}$ \\
\hline Dividende (10 Tage) & $\begin{array}{l}0,099 \\
(0,211)\end{array}$ & $\begin{array}{l}0,068 \\
(0,408)\end{array}$ & Dividende (10 Tage) & $\begin{array}{l}0,122^{* * *} \\
(0,000)\end{array}$ \\
\hline Konstante & $\begin{array}{l}0,723^{* * *} \\
(0,000)\end{array}$ & $\begin{array}{l}0,750^{* * *} \\
(0,000)\end{array}$ & Konstante & $\begin{array}{l}0,487 * * * \\
(0,000)\end{array}$ \\
\hline $\begin{array}{l}\text { Beobachtungen } \\
\text { within }-R^{2}\end{array}$ & $\begin{array}{l}18.860 \\
0,0216\end{array}$ & $\begin{array}{l}15.189 \\
0,0629\end{array}$ & & $\begin{array}{l}21.988 \\
0,0486\end{array}$ \\
\hline \multicolumn{5}{|c|}{ Panel B: 40 Tage um Ex-Tag } \\
\hline & \multicolumn{2}{|c|}{$X M L_{F}$} & & \multirow{2}{*}{$G B S_{F}$} \\
\hline & 50,000 Euros & 100,000 Euros & & \\
\hline$X L M_{A}$ & $\begin{array}{l}0,229^{* * *} \\
(0,000)\end{array}$ & $\begin{array}{l}0,956^{* *} \\
(0,016)\end{array}$ & $G B S_{A}$ & $\begin{array}{l}0,897^{* * *} \\
(0,000)\end{array}$ \\
\hline Volatilität & $\begin{array}{l}0,006^{* * *} \\
(0,001)\end{array}$ & $\begin{array}{l}0,004^{* *} \\
(0,032)\end{array}$ & Volatilität & $\begin{array}{l}0,025^{* * *} \\
(0,000)\end{array}$ \\
\hline LiqRisiko & $\begin{array}{l}0,002^{* * *} \\
(0,000)\end{array}$ & $\begin{array}{l}0,002^{* * *} \\
(0,000)\end{array}$ & LiqRisiko $_{G B S}$ & $\begin{array}{l}0,027 \\
(0,729)\end{array}$ \\
\hline Laufzeit & $\begin{array}{l}0,173^{* * *} \\
(0,000)\end{array}$ & $\begin{array}{l}0,149^{* * *} \\
(0,000)\end{array}$ & Laufzeit & $\begin{array}{l}0,121^{* * *} \\
(0,000)\end{array}$ \\
\hline Dividende (40 Tage) & $\begin{array}{l}0,095 \\
(0,121)\end{array}$ & $\begin{array}{l}0,056 \\
(0,334)\end{array}$ & Dividende (40 Tage) & $\begin{array}{l}0,286^{* * *} \\
(0,000)\end{array}$ \\
\hline Konstante & $\begin{array}{l}0,708^{* * *} \\
(0,000)\end{array}$ & $\begin{array}{l}0,742^{* * *} \\
(0,000)\end{array}$ & Konstante & $\begin{array}{l}0,441^{* * *} \\
(0,000)\end{array}$ \\
\hline $\begin{array}{l}\text { Beobachtungen } \\
\text { within }-R^{2}\end{array}$ & $\begin{array}{l}18.860 \\
0,0218\end{array}$ & $\begin{array}{l}15.189 \\
0,0634\end{array}$ & & $\begin{array}{l}21.988 \\
0,0498\end{array}$ \\
\hline
\end{tabular}

Die Tabelle zeigt die Ergebnisse der Fixed-Effects Panelregressionen mit der Liquidität des Futuresmarktes als abhängiger Variable. Die abhängige Variable der ersten beiden Regressionen ist das Liquiditätsmaß $X L M_{F}$ für ein Volumen von 50.000 Euro und 100.000 Euro. Die erklärenden Variablen sind $X L M_{S}$, Volatilität, LiqRisiko, Laufzeit und Dividende (10 Tage) (Panel A) sowie Dividende (40 Tage) (Panel B). Die abhängige Variable der dritten Regression ist die Geld-Brief-Spanne $G B S_{F}$. Die erklärenden Variablen sind $G B S_{A}$, Volatilität, LiqRisiko $G B S$, Laufzeit und Dividende (10 Tage) (Panel A) sowie Dividende (40 Tage) (Panel B). Der als Konstante ausgewiesene Wert ist der mean fixed effect. Die p-Werte sind in Klammern angegeben und wurden bestimmt durch geclusterte Standardfehler. Der Beobachtungszeitraum ist vom 01.01.2010 bis zum 31.12.2011. ${ }^{* * *},{ }^{* *}$, und ${ }^{*}$ geben die statistische Signifikanz auf dem 1\%, 5\%, and $10 \%$ Niveau an. 
Tabelle 4.21: Determinanten der Futuresliquidität: Finanzierungsliquidität

\begin{tabular}{|c|c|c|c|c|}
\hline & \multicolumn{2}{|c|}{$X M L_{F}$} & & \multirow{2}{*}{$G B S_{F}$} \\
\hline & 50,000 Euros & 100,000 Euros & & \\
\hline \multirow[t]{2}{*}{$X L M_{A}$} & $0,247^{* * *}$ & $0,927^{* *}$ & $G B S_{A}$ & $0,809^{* * *}$ \\
\hline & $(0,000)$ & $(0,016)$ & & $(0,005)$ \\
\hline \multirow[t]{2}{*}{ Volatilität } & $0,010^{* * *}$ & $0,006^{* * *}$ & Volatilität & $0,015^{* * *}$ \\
\hline & $(0,000)$ & $(0,003)$ & & $(0,000)$ \\
\hline \multirow[t]{2}{*}{ LiqRisiko } & $0,002^{* * *}$ & $0,001 * * *$ & LiqRisiko $_{G B S}$ & $-0,026$ \\
\hline & $(0,000)$ & $(0,002)$ & & $(0,833)$ \\
\hline \multirow[t]{2}{*}{ Laufzeit } & $0,179 * * *$ & $0,153^{* * *}$ & Laufzeit & $0,103^{* * *}$ \\
\hline & $(0,000)$ & $(0,000)$ & & $(0,000)$ \\
\hline \multirow[t]{2}{*}{$L I B O R$} & $-0,375^{* * *}$ & $-0,213^{* * *}$ & $L I B O R$ & $0,871^{* * *}$ \\
\hline & $(0,001)$ & $(0,006)$ & & $(0,000)$ \\
\hline \multirow[t]{2}{*}{ Konstante } & $1,018^{* * *}$ & $0,926^{* * *}$ & Konstante & $-0,129$ \\
\hline & $(0,000)$ & $(0,000)$ & & $(0,350)$ \\
\hline \multirow{2}{*}{$\begin{array}{l}\text { Beobachtungen } \\
\text { within }-R^{2}\end{array}$} & 18.860 & 15.189 & & 21.988 \\
\hline & 0,0356 & 0,0725 & & 0,0801 \\
\hline
\end{tabular}

Die Tabelle zeigt die Ergebnisse der Fixed-Effects Panelregressionen mit der Liquidität des Futuresmarktes als abhängiger Variable. Die abhängige Variable der ersten beiden Regressionen ist das Liquiditätsmaß $X L M_{F}$ für ein Volumen von 50.000 Euro und 100.000 Euro. Die erklärenden Variablen sind XLM , Volatilität, LiqRisiko, Laufzeit und LIBOR. Die abhängige Variable der dritten Regression ist die Geld-Brief-Spanne $G B S_{F}$. Die erklärenden Variablen sind $G B S A$, Volatilität, LiqRisiko ${ }_{G B S}$, Laufzeit und LIBOR. Der als Konstante ausgewiesene Wert ist der mean fixed effect. Die p-Werte sind in Klammern angegeben und wurden bestimmt durch geclusterte Standardfehler. Der Beobachtungszeitraum ist vom 01.01.2010 bis zum 31.12.2011. ***, **, und * geben die statistische Signifikanz auf dem $1 \%, 5 \%$, and $10 \%$ Niveau an.

gesetzt werden, indem eine größere Geld-Brief-Spanne gestellt wird, die keine Auswirkungen auf den Midquote hat. Durch dieses Vorgehen würden Investoren sowohl weniger SSF kaufen als auch verkaufen. Die Wirkung auf die Position des Market Makers ist damit unklar. Das Setzen einer größeren Geld-Brief-Spanne wäre lediglich sinnvoll, wenn die SSF-Position des Market Makers bereits nahe null liegt, da sie in diesem Fall als Schutz vor einer Vergrößerung der Position und den damit einhergehenden Kosten dienen würde. Folglich ist ein positiver Zusammenhang zwischen den Finanzierungskosten und der Illiquidität am SSF-Markt möglich, jedoch abhängig von der nicht beobachtbaren Position der Market Maker.

Gemessen werden die Finanzierungskosten wie im Fall der Preishypothesen durch die Variable LIBOR. In Tabelle 4.21 werden die Ergebnisse der Regressionen dargestellt. Die neue Kontrollvariable bewirkt in keiner der drei Regressionen eine Veränderung der modelltheoretischen Variablen. Ein positiver und signifikanter Einfluss der Variable $L I B O R$ wird in der Regression, die die Geld-Brief-Spanne verwendet, erkenntlich. 
In den Regressionen, die das XLM Maß verwenden, zeigt sich dagegen ein signifikant negativer Zusammenhang der Variable LIBOR mit der Illiquidität am SSF-Markt. Die Evidenz für einen Einfluss der Finanzierungskosten auf die Illiquidität am SSF-Markt ist somit als gemischt zu bezeichnen. Für die nicht eindeutige Wirkung der Finanzierungsliquidität gibt es zwei mögliche Erklärungsansätze. Erstens ist die Variable LIBOR wie bereits im Rahmen der Preishypothesen erläutert aufgrund der expansiven Geldpolitik und der damit einhergehenden geringen Zinsen möglicherweise nicht geeignet, um die Kosten der Finanzierung und deren Schwankungen adäquat zu erfassen. Zweitens kann wie im vorherigen Abschnitt erläutert keine Hypothese zu den Auswirkungen der Finanzierungsliquidität formuliert werden, die in jedem Fall Gültigkeit besitzt. Als Ergebnis kann jedoch festgehalten werden, dass die Variable LIBOR zu keinen Änderungen der modelltheoretischen Variablen führt.

Werden die Kontrollvariablen für Dividendeneffekte und Finanzierungskosten in einer Regression simultan berücksichtigt, kommt es zu keinen signifikanten Veränderungen gegenüber den Einzeleffekten. Die entsprechenden Regressionsergebnisse sind in Tabelle A.1 im Anhang dargestellt.

\subsubsection{Resultate für Indexfutures}

Zur Überprüfung der modelltheoretischen Vorhersagen sind wie in Abschnitt 4.1 dargelegt insbesondere SSF-Kontrakte geeignet. In Übereinstimmung mit dem Modell existiert für die Market Maker am SSF-Markt mit dem Basiswertpapier ein natürliches Hedginginstrument, die Marktstruktur der Eurex ist mit der im Modell unterstellten vergleichbar und die auf unterschiedliche Aktien gehandelten SSF ermöglichen es, neben den Zeitreiheneigenschaften auch den Querschnitt zu analysieren. Jedoch ist unter Verwendung von SSF-Daten aus zwei Gründen eher ein wie im Modell abgeleiteter komplementärer Zusammenhang zu erwarten. Erstens ist die Liquidität der SSF wie in Abschnitt 4.3.2 dokumentiert deutlich geringer als die der zugrundliegenden Aktien. Eine Konsequenz daraus könnte sein, dass die SSF-Kontrakte kein attraktives Substitut für die entsprechenden Basiswertpapiere darstellen. Zweitens handeln nach der Substitutionshypothese von Subrahmanyam (1991) informierte Investoren aufgrund ihrer privaten Informationen über die Einzelaktien bevorzugt am Aktienmarkt. Uninformierte Investoren dagegen weichen auf den Indexfuturesmarkt aus, um das Risiko und die Kosten adverser Selektion zu vermeiden. Dieses Argument trifft nicht auf den SSF-Markt zu, da informierte 
Tabelle 4.22: Illiquiditätsmaße der Indexfutures

\begin{tabular}{lcccccc}
\hline & \multicolumn{5}{c}{ DAX30 Indexfutures $\left(G B S_{F}^{\text {Index }}\right.$ und $X L M_{F}^{\text {Index }}$ in Prozent $)$} \\
\cline { 2 - 7 } & \multicolumn{2}{c}{ kurzfristige Indexfutures } & \multicolumn{3}{c}{ langfristige Indexfutures } \\
\cline { 2 - 7 } & Mittelwert & Median & $\mathrm{N}$ & Mittelwert & Median & $\mathrm{N}$ \\
\hline Geld-Brief-Spanne & 0,0136 & 0,0140 & 495 & 0,0224 & 0,0221 & 495 \\
XLM 250.000 Euro & 0,0871 & 0,0120 & 506 & 1,1796 & 0,8280 & 479 \\
XLM 500.000 Euro & 0,1201 & 0,0139 & 506 & 1,5244 & 1,1619 & 450 \\
\hline
\end{tabular}

Diese Tabelle zeigt den Mittelwert, den Median sowie die Anzahl der täglichen Beobachtungen (N) der Liquiditätsmaße $G B S_{F}^{\text {Index }}$ und $X L M_{F}^{\text {Index }}$ der kurz- und langfristigen Indexfutures. Der Beobachtungszeitraum ist vom 01.01.2010 bis zum 31.12.2011.

Investoren keine natürliche Präferenz haben, im Markt des Basiswertpapiers zu handeln. Im Gegenteil ist es möglich, durch SSF-Positionen einen höheren Verschuldungsgrad zu erreichen.

Folglich ist es von Interesse einen Datensatz zu analysieren für den das Substitutionsargument stärker ist und zu untersuchen, ob dies den erwarteten Effekt hat. Daher werden in der weiteren Analyse Indexfutures anstelle der SSF-Daten verwendet. Die abhängigen Variablen sind die XLM Maße $\left(X L M_{F}^{\text {Index }}\right)$ und die Geld-Brief-Spanne $\left(G B S_{F}^{\text {Index }}\right)$ des DAX30 Indexfutures. Die Variable $X L M_{F}^{\text {Index }}$ bezieht sich dabei auf ein Volumen von 250.000 und 500.000 Euro. Diese Volumina werden verwendet, da ein Euro Betrag von 50.000 bzw. 100.000 Euro nicht ausreichend ist, um einen DAX30 Indexfutureskontrakt zu kaufen. Wie in der vorherigen Analyse werden die Futureskontrakte mit der kürzesten und zweitkürzesten Laufzeit analysiert, wobei Beobachtungen im Verfallsmonat ausgeschlossen werden. Auch negative Geld-Brief-Spannen und Beobachtungen von über 1000 BP werden weiterhin nicht in der Analyse berücksichtigt.

Tabelle 4.22 zeigt deskriptive Statistiken der Illiquiditätsmaße des Indexfutures. Mit einer mittleren Geld-Brief-Spanne von 0,0136\% ist der Indexfuture als sehr liquide zu bezeichnen. Der Preiseinfluss für ein Volumen von 250.000 bzw. 500.000 Euro ist mit 0,0871 \% bzw. 0,1201\% entsprechend höher, jedoch immer noch deutlich geringer als der der SSF. Auch für den Indexfutures sind die Mittelwerte der Illiquiditätsmaße deutlich größer für langfristige als für kurzfristige Kontrakte.

Die erklärenden Variablen werden analog zum Basisfall definiert. Die Liquidität des Aktienmarktes wird dabei als arithmetisches Mittel der XLM Maße $\left(X L M_{A}^{\text {Index }}\right)$ und Geld-Brief-Spannen $\left(G B S_{A}^{\text {Index }}\right)$ der DAX30 Aktien gemessen. Das Preisrisiko (Volatili- 
tät $\left.t^{\text {Index }}\right)$ wird durch die annualisierte Standardabweichung der täglichen DAX30 Renditen über ein rollierendes Fenster der letzten 30 Handelstage berechnet. Das Liquiditätsrisiko (LiqRisiko ${ }^{\text {Index }}$ bzw. LiqRisiko $o_{G B S}^{\text {Index }}$ ) wird durch die annualisierte Standardabweichung der Variablen $X L M_{F}^{I n d e x}$ bzw. $G B S_{F}^{I n d e x}$ über ein rollierendes Fenster der letzten 30 Handelstage berechnet. Zur Überprüfung der Laufzeiteffekte wird analog zum bisherigen Vorgehen wieder eine Dummy Variable verwendet und der Beobachtungszeitraum reicht weiterhin vom 01.01.2010 bis zum 31.12.2011.

Die Regressionsergebnisse des DAX30 Indexfutures sind in Tabelle 4.23 abgebildet und stimmen mit einer bedeutenden Ausnahme mit den Ergebnissen der SSF überein. Die Illiquidität des Indexfutures gemessen durch die Variable $X L M_{F}^{I n d e x}$ steht wie in der vorangegangenen Analyse in einem positiven und signifikanten Zusammenhang zum Preisund Liquiditätsrisiko am Aktienmarkt. Auch wenn die Illiquidität durch die Geld-BriefSpanne gemessen wird, kann in Übereinstimmung mit dem Basisfall ein positiver Einfluss des Preisrisikos, jedoch kein Einfluss des Liquiditätsrisikos auf die Illiquidität am Indexfuturesmarkt nachgewiesen werden. Wie bereits erläutert, stimmt die Insignifikanz der Variable LiqRisiko $O_{G B S}^{I n d e x}$ mit den Modellvorhersagen überein, da ein messbarer Preiseinfluss für das Liquiditätsrisiko lediglich für größere Wert von $H_{*}$ entstehen sollte (vgl. Abbildung 3.6). Ebenfalls in Übereinstimmung mit den SSF Resultaten lässt sich für beide Illiquiditätsmaße eine signifikant höhere Illiquidität von langfristigen Indexfutures gegenüber kurzfristigen Indexfutures nachweisen.

Der wesentliche Unterschied zu den Ergebnissen der SSF liegt darin, dass die Bedeutung der Illiquidität am Aktienmarkt geringer ist. Wird die Liquidität anhand der Geld-BriefSpanne gemessen, kann weiterhin ein positiver signifikanter Einfluss der Illiquidität des Aktienmarktes auf die Illiquidität am Futuresmarkt nachgewiesen werden. Wird die Illiquidität jedoch durch das XLM gemessen, kann weder für ein Volumen von 250.000 noch von 500.000 Euro ein signifikanter Zusammenhang gefunden werden. Dieses Ergebnis kann durch zwei unterschiedliche Argumente erklärt werden. Nach dem ersten bereits erläuterten Argument besitzen informierte Investoren private Informationen über die Einzelaktien und handeln daher bevorzugt am Aktienmarkt. Uninformierte Investoren dagegen werden auf dem Indexfuturesmarkt handeln, sofern die Kosten adverser Selektion am Aktienmarkt hoch sind (Subrahmanyam (1991)). Dieses Verhalten führt zu einer inversen Beziehung von Illiquidität am Aktien und Indexfuturesmarkt. Diesem Effekt wirkt das Verhalten der Market Maker entgegen, welche sich gegenüber ihren Positionen am Indexfuturesmarkt absichern und dadurch eine gleichgerichtete Bewegung 
Tabelle 4.23: Determinanten der Futuresliquidität: Indexfutures

\begin{tabular}{|c|c|c|c|c|}
\hline & \multicolumn{2}{|c|}{$X L M_{F}^{I n d e x}$} & & \multirow{2}{*}{$G B S_{F}^{I n d e x}$} \\
\hline & 250.000 Euros & 500.000 Euros & & \\
\hline$X L M_{A}^{I n d e x}$ & $\begin{array}{r}-0,4791 \\
(0,695)\end{array}$ & $\begin{array}{l}0,4673 \\
(0,612)\end{array}$ & $G B S_{A}^{\text {Index }}$ & $\begin{array}{l}3,9214^{* * *} \\
(0,000)\end{array}$ \\
\hline Volatilität ${ }^{\text {Index }}$ & $\begin{array}{l}0,0186^{* * *} \\
(0,000)\end{array}$ & $\begin{array}{l}2,0249^{* *} \\
(0,000)\end{array}$ & Volatilität Index & $\begin{array}{l}0,0003^{* * *} \\
(0,000)\end{array}$ \\
\hline LiqRisiko Index & $\begin{array}{l}0,6971^{* * *} \\
(0,001)\end{array}$ & $\begin{array}{l}0,5536^{* * *} \\
(0,001)\end{array}$ & LiqRisiko $\underset{G B S}{I n d e x}$ & $\begin{array}{r}-0,1527 \\
(0,366)\end{array}$ \\
\hline Laufzeit & $\begin{array}{l}1,1154^{* * *} \\
(0,000)\end{array}$ & $\begin{array}{l}1,4146^{* * *} \\
(0,000)\end{array}$ & Laufzeit & $\begin{array}{l}0,0086^{* * *} \\
(0,000)\end{array}$ \\
\hline Konstante & $\begin{array}{c}-0,4224^{* * *} \\
(0,003)\end{array}$ & $\begin{array}{c}-0,6674^{* * *} \\
(0,000)\end{array}$ & Konstante & $\begin{array}{l}0,0046^{* * *} \\
(0,000)\end{array}$ \\
\hline $\begin{array}{l}\text { Beobachtungen } \\
\text { adj. }-R^{2}\end{array}$ & $\begin{array}{c}917 \\
0,3564\end{array}$ & $\begin{array}{c}888 \\
0,4169\end{array}$ & & $\begin{array}{c}930 \\
0,5162\end{array}$ \\
\hline
\end{tabular}

Die Tabelle zeigt die Ergebnisse der Regressionen mit der Liquidität des Indexfuturesmarktes als abhängiger Variable. Die abhängige Variable der ersten beiden Regressionen ist das Liquiditätsmaß $X L M_{F}^{I n d e x}$ für ein Volumen von 250.000 Euro und 500.000 Euro. Die erklärenden Variablen sind XLM $M_{A}^{\text {Index }}$, Volatilität ${ }^{\text {Index }}$, LiqRisiko ${ }^{\text {Index }}$, und Laufzeit. Die abhängige Variable der dritten Regression ist die Geld-Brief-Spanne $G B S_{F}^{\text {Index }}$. Die erklärenden Variablen sind $G B S_{A}^{\text {Index }}$, Volatilität ${ }^{\text {Index }}$, LiqRisiko ${ }_{G B S}^{\text {Index }}$, und Laufzeit. Der als Konstante ausgewiesene Wert ist der mean fixed effect. Die p-Werte sind in Klammern angegeben und wurden bestimmt durch robuste Standardfehler nach White (1980). Der Beobachtungszeitraum ist vom 01.01.2010 bis zum 31.12.2011. ***, **, und * geben die statistische Signifikanz auf dem $1 \%, 5 \%$, and $10 \%$ Niveau an.

der Illiquidität auf beiden Märkten bewirken. Sofern die Ausprägung dieser beiden Effekte vergleichbar ist, werden sie sich aufheben, was dazu führt, dass keine Beziehung nachweisbar ist. Es bleibt somit festzuhalten, dass die Substitutionshypothese und die „derivative-hedge-theory“ sich nicht gegenseitig ausschließen. Der zweite mögliche Grund für die Insignifikanz der Variable $X L M_{F}^{\text {Index }}$ ist, dass die Markt Maker ihre Indexfuturespositionen nicht am Aktienmarkt sondern am ETF-Markt hedgen. Da das XLM Maß für den ETF-Markt jedoch nicht verfügbar ist, kann die Hypothese, dass die Illiquidität von Indexfutures und ETFs in einem positiven Zusammenhang steht, nicht überprüft werden. 


\section{Schlussbetrachtung und Ausblick}

Ziel der vorliegenden Arbeit ist es, ein besseres Verständnis der Liquiditätszusammenhänge von Kassa- und Derivatemärkten zu gewinnen und mit der Fokussierung auf Futuresmärkte eine bestehende Lücke in der wissenschaftlichen Literatur zu schließen. In einer friktionslosen Welt sind die Preise auf Kassa- und Futuresmärkten durch die Costof-Carry-Formel verbunden. In der Realität sind diese Märkte jedoch durch Friktionen wie Illiquidität gekennzeichnet. Aufgrund der engen Verbindung von Kassa- und Futurespreisen ist davon auszugehen, dass auch die Illiquidität beider Mäkte in einer engen Beziehung steht und die Illiquidität des Kassamarktes die Abweichung der Futurespreise von den Cost-of-Carry-Preisen beeinflusst. Daher untersucht die vorliegende Arbeit die Auswirkungen der Illiquidität am Kassamarkt auf die Preise und Illiquidität am Futuresmarkt. Die Analyse erfolgt dabei im Rahmen eines theoretischen Modells sowie in einer empirischen Untersuchung.

Zunächst wird ein Modell entwickelt, das als Formalisierung der „derivative-hedge-theorie“" von Cho \& Engle (1999) verstanden werden kann. Anders als in der bestehenden Literatur wird sowohl die Illiquidität am Kassa- als auch am Futuresmarkt berücksichtigt. Das Modell geht von Wettbewerb unter Market Makern aus und beschreibt das Verhalten eines repräsentativen Market Makers. Dieser bedient die exogene Nachfrage nach Futures und kann sich über den Kassamarkt absichern. Aufgrund der Illiquidität am Kassamarkt verursacht das Absichern Kosten. Der Market Maker wägt zwischen diesen Kosten und den Risiken aus seiner offenen Futuresposition ab. Eine analytische Lösung des Modells wird für einen repräsentativen Market Maker mit CARA-Nutzenfunktion bestimmt. Im Optimum hält der Market Maker eine Kassamarktposition, die sich aus einer Komponente, die zur Absicherung dient, und aus einer Komponente, die er zu spekulativen Zwecken hält, zusammensetzt. Je höher die Illiquidität am Kassamarkt und damit die Hedgingkosten des Market Makers, umso weniger sichert er sich gegen die Risiken aus seiner Futuresposition ab. Für die Futurespreise kann eine Bewertungsgleichung in Abhängigkeit der Nachfrage bestimmt werden.

Die Bestimmung der Futurespreise für eine spezielle Nachfrage ermöglicht es, Hypothesen zur Abweichung der Futurespreise von den Cost-of-Carry-Preisen abzuleiten. Die Illiquidität am Kassamarkt und die Laufzeit der Kontrakte erhöhen die Risikoaversion des Market Makers und die Volatilität am Kassamarkt verringert den Absolutbetrag 
der Preisabweichungen. Diese Resultate sind auf die spekulative Komponente des Market Makers zurückzuführen. Die Auswirkungen der Risikoaversion des Market Makers und der Volatilität am Kassamarkt sind dadurch zu erklären, dass ein Anstieg beider Faktoren zu weniger Spekulation des Market Makers und damit zu einer geringeren Abweichung vom Cost-of-Carry-Preis führt.

Die Sensitivität der Futurespreise bzgl. der Nachfrage dient als Liquiditätsmaß, unter dessen Verwendung Hypothesen zur Liquiditätsübertragung abgeleitet werden. Zusammenfassend zeigt sich, dass die Illiquidität am Futuresmarkt in einem positiven Zusammenhang zu der Illiquidität, der Volatilität und dem Liquiditätsrisiko am Kassamarkt sowie der Risikoaversion des Market Makers und der Laufzeit der Kontrakte steht. Die Effekte der einzelnen Einflussfaktoren sind in der Modellvariante mit Liquiditätsrisiko stärker ausgeprägt je größer die Nachfrage nach Long- oder Shortpositionen am Futuresmarkt ist. Ein Anstieg der Korrelation von Preis- und Liquiditätsrisiko sowie der erwarteten Rendite führt bei einer Nachfrage nach Longpositionen (Shortpositionen) $\mathrm{zu}$ einer steigenden (sinkenden) Illiquidität. Dabei ist die ökonomische Bedeutung der erwarteten Rendite gering. Darüber hinaus lassen sich anhand verschiedener Modellvarianten weitere Aspekte der Liquiditätsübertragung analysieren. So können die Effekte der Liquiditätsdimensionen Markttiefe und Marktenge separiert werden. Die Marktenge führt dazu, dass sich der Market Maker bei einer geringen Nachfrage aufgrund der zu hohen Kosten für die erste Einheit nicht absichert, wodurch die Auswirkungen der Kassamarktilliquidität besonders stark sind. Es kann außerdem gezeigt werden, dass sich eine asymmetrische Form des Orderbuchs am Kassamarkt auf den Futuresmarkt überträgt. Diese Modellvariante ermöglicht es auch, die Auswirkungen von Leerverkaufsverboten als Spezialfall zu untersuchen. Bei einem Leerverkaufsverbot reagieren die Futurespreise besonders sensitiv auf eine Veränderung in der Nachfrage nach Shortpositionen. Im Rahmen des Mehrperiodenmodells können die Liquiditätsunterschiede von Futures mit unterschiedlichen Verfallszeitpunkten explizit analysiert sowie die Handelsstrategie des Market Makers genauer untersucht werden. Es wird nachgewiesen, dass der Market Maker lediglich zu Beginn am Kassamarkt handelt und es danach zu keiner Anpassung seiner Position kommt.

Die Arbeit ist die erste, die Preis- und Liquiditätseffekte am Futuresmarkt anhand von Aktien und der darauf gehandelten SSF analysiert. Konkret wird ein Paneldatensatz des DAX30 verwendet. Die empirische Untersuchung findet starke Evidenz dafür, dass eine höhere Illiquidität sowie Volatilität am Kassamarkt und eine längere Restlaufzeit der 
Kontrakte die Preisabweichungen am SSF-Markt verstärken. Die modelltheoretischen Vorhersagen bzgl. Illiquidität am Kassamarkt und Laufzeit der Kontrakte können folglich bestätigt werden. Das Ergebnis zur Volatilität ist im Kontext eines risikoaversen Market Makers ökonomisch plausibel. Es kommt zu keinen qualitativen Änderungen, wenn in den Regressionen simultan für das Liquiditätsrisiko am Kassamarkt, die Nachfrageeffekte aufgrund von Dividendenstrategien, im Zeitablauf schwankende Finanzierungskosten des Market Makers und die Illiquidität am Futuresmarkt kontrolliert wird, womit die Ergebnisse als sehr robust zu bezeichnen sind.

Die Ergebnisse der empirischen Analyse stützen ebenfalls die modelltheoretischen Vorhersagen zur Liquiditätsübertragung. Es zeigt sich, dass die Illiquidität am SSF-Markt mit der Illiquidität, der Volatilität und dem Liquiditätsrisiko am Kassamarkt sowie der Restlaufzeit der Kontrakte steigt. Die Ergebnisse sind robust, wenn alternative Liquiditäts- und Risikomaße verwendet werden sowie wenn für Nachfrageeffekte aufgrund von Dividendenstrategien und im Zeitablauf schwankende Finanzierungskosten des Market Makers kontrolliert wird. Die Resultate sind somit konsistent mit der „derivative-hedge-theory“. Gleichzeitig sind die Ergebnisse nicht vereinbar mit der Substitutionshypothese, die unterstellt, dass Aktien und Futures Substitute sind und die Liquidität in einem negativen Zusammenhang stehen sollte. Die Analyse wird darüber hinaus für Daten der DAX30 Indexfutures durchgeführt. Für diesen Datensatz sollte das Substitutionsargument von Subrahmanyam (1991) eine größere Bedeutung haben. Tatsächlich zeigt sich, dass die Illiquidität am Kassamarkt keinen signifikanten Einfluss auf die Illiquidität des Indexfutures hat. Die Bedeutung der übrigen Variablen bleibt weiterhin bestehen. Die Insignifikanz der Illiquidität am Kassamarkt kann dadurch erklärt werden, dass sich der Substitutions- und Hedgingeffekt aufheben. Eine andere mögliche Erklärung ist, dass sich die Market Maker nicht am Aktienmarkt gegenüber den Risiken aus ihrer Indexfuturesposition absichern, sondern über den ETF-Markt.

Die vorliegende Arbeit zeigt verschiedene Möglichkeiten für zukünftige Forschung auf. So liegt der Fokus des Modells nicht auf der asymmetrischen Information, die im Rahmen der Liquiditätsforschung eine bedeutende Rolle einnimmt. Es stellt sich daher die Frage der Integration dieser Friktion. Eine Weiterentwicklung des Modells könnte insofern die Effekte von Hedgingkosten, Inventarrisiken und asymmetrischer Information simultan analysieren. Als Ausgangspunkt zur Modellierung könnte eine positive Abhängigkeit zwischen der erwarteten Rendite am Kassamarkt und der Nachfrage nach Longpositionen am Futuresmarkt unterstellt werden. In diesem Fall würde der Market Maker aus 
der Nachfrage Informationen über die erwartete Rendite am Kassamarkt ableiten und in seinem Portfoliokalkül berücksichtigen, was die Ableitung weiterer empirisch testbarer Hypothesen zu den Preis- und Liquiditätseffekten am Futuresmarkt ermöglichen würde. Das Modell könnte außerdem auf nichtlineare Derivate wie Optionen übertragen werden. Im Rahmen des Mehrperiodenmodells konnte gezeigt werden, dass der Market Maker zur Absicherung seiner Futurespositionen eine statische Hedgingstrategie verfolgt und folglich keine Kosten durch die Umschichtung seiner Kassamarktposition anfallen. Die Studie von Goyenko et al. (2014) zeigt jedoch, dass bei der Absicherung gegenüber Risiken aus Optionspositionen den aus der Umschichtung resultierenden Kosten eine bedeutende Rolle zukommt. Folglich ist davon auszugehen, dass der Market Maker am Optionsmarkt anderen Hedgingkosten ausgesetzt ist und es folglich auch zu Unterschieden in der Liquiditätsübertragung kommt. 


\section{Literaturverzeichnis}

Acharya, V. V. \& Pedersen, L. H. (2005), 'Asset pricing with liquidity risk', Journal of Financial Economics 77(2), 375-410.

Admati, A. R. \& Pfleiderer, P. (1988), 'A theory of intraday patterns: Volume and price variability', Review of Financial Studies 1(1), 3-40.

Amihud, Y. (2002), 'Illiquidity and stock returns: Cross-section and time-series effects', Journal of Financial Markets 5(1), 31-56.

Amihud, Y. \& Mendelson, H. (1980), 'Dealership market: Market-making with inventory', Journal of Financial Economics 8(1), 31-53.

Amihud, Y. \& Mendelson, H. (1986), 'Liquidity and stock returns', Financial Analysts Journal 42(3), 43-48.

Amihud, Y. \& Mendelson, H. (1991), 'Liquidity, maturity, and the yields on US treasury securities', The Journal of Finance 46(4), 1411-1425.

Amihud, Y., Mendelson, H. \& Pedersen, L. H. (2005), 'Liquidity and asset prices', Foundations and Trends in Finance 1(4), 269-364.

Back, K., Cao, C. H. \& Willard, G. A. (2000), 'Imperfect competition among informed traders', The Journal of Finance 55(5), 2117-2155.

Bagehot, W. (1971), 'The only game in town', Financial Analysts Journal 27(2), 12-14.

Battalio, R. \& Schultz, P. (2011), 'Regulatory uncertainty and market liquidity: The 2008 short sale ban's impact on equity option markets', The Journal of Finance 66(6), 20132053.

Baule, R. (2011), 'The order flow of discount certificates and issuer pricing behavior', Journal of Banking \& Finance 35(11), 3120-3133.

Bekaert, G., Harvey, C. R. \& Lundblad, C. (2007), 'Liquidity and expected returns: Lessons from emerging markets', Review of Financial Studies 20(6), 1783-1831.

Bekaert, G., Hoerova, M. \& Lo Duca, M. (2013), 'Risk, uncertainty and monetary policy', Journal of Monetary Economics 60(7), 771-788. 
Bessembinder, H. \& Seguin, P. J. (1993), 'Price volatility, trading volume, and market depth: Evidence from futures markets', Journal of Financial and Quantitative Analysis 28(1), 21-39.

Bialkowski, J. \& Jakubowski, J. (2012), 'Determinants of trading activity on the singlestock futures market: Evidence from the Eurex exchange', Jounal of Derivatives 19(3), 29-47.

Black, F. \& Scholes, M. (1973), 'The pricing of options and corporate liabilities', Journal of Political Economy 81(3), 637-654.

Blau, B. M. \& Wade, C. (2013), 'Comparing the information in short sales and put options', Review of Quantitative Finance and Accounting 41(3), 567-583.

Bollen, N. P. B. \& Whaley, R. E. (2004), 'Does net buying pressure affect the shape of implied volatility functions?', The Journal of Finance 59(2), 711-753.

Bollen, N. P., Smith, T. \& Whaley, R. E. (2004), 'Modeling the bid/ask spread: Measuring the inventory-holding premium', Journal of Financial Economics 72(1), 97-141.

Boyle, P. P. \& Vorst, T. (1992), 'Option replication in discrete time with transaction costs', The Journal of Finance 47(1), 271-293.

Calamia, A., Deville, L. \& Riva, F. (2014), The determinants of ETF liquidity: Theory and evidence from European markets, Working paper, CNRS and EDHEC Business School.

Cao, C., Zhiwu, C. \& Griffin, J. M. (2005), 'Informational content of option volume prior to takeovers', Journal of Business 78(3), 1073-1109.

Cespa, G. \& Foucault, T. (2014), 'Illiquidity contagion and liquidity crashes', Review of Financial Studies 27(6), 1615-1660.

Cetin, U., Jarrow, R., Protter, P. \& Warachka, M. (2006), 'Pricing options in an extended Black Scholes economy with illiquidity: Theory and empirical evidence', Review of Financial Studies 19(2), 493-529.

Cho, Y.-H. \& Engle, R. F. (1999), Modeling the impacts of market activity on bid-ask spreads in the option market, Working paper, University of California. 
Chou, R. K., Chung, S.-L., Hsiao, Y.-J. \& Wang, Y.-H. (2011), 'The impact of liquidity on option prices', Journal of Futures Markets 31(12), 1116-1141.

Christoffersen, P., Goyenko, R., Jacobs, K. \& Karoui, M. (2014), Illiquidity premia in the equity options market, Working paper, University of Toronto.

Copeland, T. E. \& Galai, D. (1983), 'Information effects on the bid-ask spread', The Journal of Finance 38(5), 1457-1469.

Corwin, S. A. \& Schultz, P. (2012), 'A simple way to estimate bid-ask spreads from daily high and low prices', The Journal of Finance 67(2), 719-760.

Cox, J. C., Ingersoll, J. E. \& Ross, S. A. (1981), 'The relation between forward prices and futures prices', Journal of Financial Economics 9(4), 321-346.

Damodaran, A. \& Lim, J. (1991), 'The effects of option listing on the underlying stocks' return processes', Journal of Banking \& Finance 15(3), 647-664.

Danielsen, B. R. \& Sorescu, S. M. (2001), 'Why do option introductions depress stock prices? A study of diminishing short sale constraints', Journal of Financial and Quantitative Analysis 36(4), 451-484.

Danielsen, B. R., Van Ness, R. A. \& Warr, R. S. (2009), 'Single-stock futures as a substitute for short sales: Evidence from microstructure data', Journal of Business Finance \& Accounting 36(9-10), 1273-1293.

De Jong, F. \& Rindi, B. (2009), The microstructure of financial markets, Cambridge University Press.

De Roon, F. A., Nijman, T. E. \& Veld, C. (2000), 'Hedging pressure effects in futures markets', The Journal of Finance 55(3), 1437-1456.

Easley, D. \& O'Hara, M. (1987), 'Price, trade size, and information in securities markets', Journal of Financial Economics 19(1), 69-90.

Easley, D., O'Hara, M. \& Srinivas, P. S. (1998), 'Option volume and stock prices: Evidence on where informed traders trade', The Journal of Finance 53(2), 431-465.

Elstrodt, J. (2005), Maß- und Integrationtheorie, 4. Aufl., Springer.

Engle, R. \& Neri, B. (2010), The impact of hedging costs on the bid and ask spread in the options market, Working paper, New York University. 
Eurex (2014), 'Single stock futures at Eurex exchange'. http://www . eurexchange.com/ exchange-en/products/equ/fut/.

Eurex (2015), 'Eurex Exchange Marktmodell'. http://www.eurexchange.com/ exchange-de/handel/marktmodell.

Fedenia, M. \& Grammatikos, T. (1992), 'Options trading and the bid-ask spread of the underlying stocks', Journal of Business 65(3), 335-351.

Figlewski, S. \& Webb, G. P. (1993), 'Options, short sales, and market completeness', The Journal of Finance 48(2), 761-777.

Fong, K., Holden, C. \& Trzcinka, C. (2014), What are the best liquidity proxies for global research, Working paper, University of New South Wales and Indiana University.

Foster, F. D. \& Viswanathan, S. (1996), 'Strategic trading when agents forecast the forecasts of others', The Journal of Finance 51(4), 1437-1478.

Foucault, T., Kadan, O. \& Kandel, E. (2005), 'Limit order book as a market for liquidity', Review of Financial Studies 18(4), 1171-1217.

Frey, R. (1998), 'Perfect option hedging for a large trader', Finance and Stochastics $2(2), 115-141$.

Frey, R. (2000), Market illiquidity as a source of model risk in dynamic hedging, Working paper, University of Zürich.

Garman, M. B. (1976), 'Market microstructure', Journal of Financial Economics $3(3), 257-275$.

George, T., Kaul, G. \& Nimalendran, M. (1991), 'Estimation of the bid-ask spread and its components: a new approach', Review of Financial Studies 4(4), 623-656.

Glosten, L. R. \& Milgrom, P. R. (1985), 'Bid, ask and transaction prices in a specialist market with heterogeneously informed traders', Journal of Financial Economics 14(1), 71-100.

Gomber, P. \& Schweickert, U. (2002), 'Der Market Impact: Liquiditätsmaß im elektronischen Wertpapierhandel', Die Bank 7, 485-489. 
Gomber, P., Schweickert, U. \& Theissen, E. (2015), 'Liquidity dynamics in an electronic open limit order book: An event study approach', European Financial Management $\mathbf{2 1}(1), 52-78$.

Goyenko, R., Ortahanlai, C. \& Tang, S. (2014), Trading cost dynamics of market making in equity options, Working paper, McGill University.

Goyenko, R. Y., Holden, C. W. \& Trzcinka, C. A. (2009), 'Do liquidity measures measure liquidity?', Journal of Financial Economics 92(2), 153-181.

Gârleanu, N., Pedersen, L. H. \& Poteshman, A. M. (2009), 'Demand-based option pricing', Review of Financial Studies 22(10), 4259-4299.

Grundy, B. D., Lim, B. \& Verwijmeren, P. (2012), 'Do option markets undo restrictions on short sales? Evidence from the 2008 short-sale ban', Journal of Financial Economics 106(2), 331-348.

Hasbrouck, J. (2006), Empirical market microstructure: The institutions, economics, and econometrics of securities trading, Oxford University Press.

Hasbrouck, J. (2009), 'Trading costs and returns for US equities: Estimating effective costs from daily data', The Journal of Finance 64(3), 1445-1477.

Hasbrouck, J. \& Schwartz, R. A. (1988), 'Liquidity and execution costs in equity markets', The Journal of Portfolio Management 14(3), 10-16.

Hirshleifer, D. (1989), 'Determinants of hedging and risk premia in commodity futures markets', Journal of Financial and Quantitative Analysis 24(3), 313-331.

Ho, T. S. \& Stoll, H. R. (1983), 'The dynamics of dealer markets under competition', The Journal of Finance 38(4), 1053-1074.

Ho, T. \& Stoll, H. R. (1981), 'Optimal dealer pricing under transactions and return uncertainty', Journal of Financial Economics 9(1), 47-73.

Holden, C. W. \& Subrahmanyam, A. (1992), 'Long-lived private information and imperfect competition', The Journal of Finance 47(1), 247-270.

Huang, R. \& Stoll, H. (1997), 'The components of the bid-ask spread: A general approach', Review of Financial Studies 10(4), 995-1034. 
Irvine, P., Benston, G. \& Kandel, E. (2000), Liquidity beyond the inside spread: Measuring and using information in the limit order book, Working paper, Neeley School of Business.

Jegadeesh, N. \& Subrahmanyam, A. (1993), 'Liquidity effects of the introduction of the S\&P 500 index futures contract on the underlying stocks', The Journal of Business 66(2), 171-87.

Jones, C. M. \& Lipson, M. L. (2001), 'Sixteenths: Direct evidence on institutional execution costs', Journal of Financial Economics 59(2), 253-278.

Kadapakkam, P.-R. \& Kumar, U. (2013), 'Impact of liquidity on the futures-cash basis: Evidence from the Indian market', Journal of Futures Markets 33(3), 266-298.

Karakaya, M. (2014), Characteristics and expected returns in individual equity options, Working paper, University of Chicago.

Kaul, G., Nimalendran, M. \& Zhang, D. (2004), Informed trading and option spreads, Working paper, University of Michigan.

Korajczyk, R. A. \& Sadka, R. (2008), 'Pricing the commonality across alternative measures of liquidity', Journal of Financial Economics 87(1), 45-72.

Korn, O., Krischak, P. \& Theissen, E. (2015), Illiquidity transmission from spot to futures markets, Working paper, Georg-August-Universität Göttingen.

Kumar, R., Sarin, A. \& Shastri, K. (1995), 'The impact of index options on the underlying stocks: The evidence from the listing of Nikkei stock average options', Pacific-Basin Finance Journal 3(2), 303-317.

Kumar, R., Sarin, A. \& Shastri, K. (1998), 'The impact of options trading on the market quality of the underlying security: An empirical analysis', The Journal of Finance 53(2), 717-732.

Kyle, A. S. (1985), 'Continuous auctions and insider trading', Econometrica 53(6), 13151335 .

Landsiedl, F. (2005), The market microstructure of illiquid options markets and interrelations with the underlying market, Working paper, Vienna University of Economics and Business Administration. 
Leland, H. E. (1985), 'Option pricing and replication with transactions costs', The Journal of Finance 40(5), 1283-1301.

Lien, D., Lim, G., Yang, L. \& Zhou, C. (2013), 'Dynamic dependence between liquidity and the S\&P 500 index futures-cash basis', Journal of Futures Markets 33(4), 327-342.

Liu, H. \& Yong, J. (2005), 'Option pricing with an illiquid underlying asset market', Journal of Economic Dynamics and Control 29(12), 2125-2156.

Madhavan, A. \& Smidt, S. (1991), 'A Bayesian model of intraday specialist pricing', Journal of Financial Economics 30(1), 99-134.

Manaster, S. \& Mann, S. C. (1996), 'Life in the pits: Competitive market making and inventory control', Review of Financial Studies 9(3), 953-975.

Menyah, K. \& Paudyal, K. (2000), 'The components of bid-ask spreads on the london stock exchange', Journal of Banking \&6 Finance 24(11), 1767-1785.

Merton, R. C. (1980), 'On estimating the expected return on the market: An exploratory investigation', Journal of Financial Economics 8(4), 323-361.

O'Hara, M. (2008), Market microstructure theory, Cambridge, MA: Blackwell Publishers.

O'Hara, M. \& Oldfield, G. S. (1986), 'The microeconomics of market making', Journal of Financial and Quantitative Analysis 21(4), 361-376.

Pastor, L. \& Stambaugh, R. F. (2003), 'Liquidity risk and expected stock returns', Journal of Political Economy 111(3), 642-685.

Petersen, M. A. (2009), 'Estimating standard errors in finance panel data sets: Comparing approaches', Review of Financial Studies 22(1), 435-480.

Petrella, G. (2006), 'Option bid-ask spread and scalping risk: Evidence from a covered warrants market', Journal of Futures Markets 26(9), 843-867.

Roll, R. (1984), 'A simple implicit measure of the effective bid-ask spread in an efficient market', The Journal of Finance 39(4), 1127-1139.

Roll, R., Schwartz, E. \& Subrahmanyam, A. (2007), 'Liquidity and the law of one price: The case of the futures-cash basis', The Journal of Finance 62(5), 2201-2234. 
Schestag, R., Schuster, P. \& Uhrig-Homburg, M. (2013), Measuring liquidity in bond markets, Working paper, Karlsruhe Institute of Technology.

Seppi, D. J. (1990), 'Equilibrium block trading and asymmetric information', The Journal of Finance 45(1), 73-94.

Spiegel, M. \& Subrahmanyam, A. (1992), 'Informed speculation and hedging in a noncompetitive securities market', Review of Financial Studies 5(2), 307-329.

Stoll, H. R. (1978), 'The supply of dealer services in securities markets', The Journal of Finance 33(4), 1133-1151.

Stoll, H. R. (1979), 'Commodity futures and spot price determination and hedging in capital market equilibrium', Journal of Financial and Quantitative Analysis 14(4), 873894.

Stoll, H. R. (1989), 'Inferring the components of the bid-ask spread: Theory and empirical tests', Journal of Finance 44(1), 115-134.

Subrahmanyam, A. (1991), 'A theory of trading in stock index futures', Review of Financial Studies 4(1), 17-51.

Vijh, A. M. (1990), 'Liquidity of the CBOE equity options', The Journal of Finance 45(4), 1157-1179.

Watanabe, A. \& Watanabe, M. (2008), 'Time-varying liquidity risk and the cross section of stock returns', Review of Financial Studies 21(6), 2449-2486.

White, H. (1980), 'A heteroskedasticity-consistent covariance matrix estimator and a direct test for heteroskedasticity', Econometrica 48(4), 817-838.

Xetra (2015), 'Marktmodell Aktien'. http://www.xetra. com/blob/1208450/d0fd5549a52dab434f91ef0f73a12b2c/data/ Marktmodell-Aktien-Xetra-Release-16.0.pdf.

Yadav, P. K. \& Pope, P. F. (1994), 'Stock index futures mispricing: Profit opportunities or risk premia?', Journal of Banking \& Finance 18(5), 921-953. 


\section{A Anhang}

\section{A.1 Liquiditätsrisiko am Kassamarkt: Herleitung des Forwardpreises und der optimalen Kassamarktposition des Market Makers}

Das Optimierungsproblem des Market Makers ist gegeben als:

$$
\max _{H_{A}, X_{0}} E_{0}\left[\left(\widetilde{W}_{1}\right)\right]-\frac{1}{2} A \operatorname{Var}_{0}\left[\left(\widetilde{W}_{1}\right)\right]
$$

Unter Verwendung der Gleichung (3.25) für $\widetilde{W}_{1}$ sowie der gemeinsamen Verteilung von $\widetilde{P}_{1}$ und $\tilde{b}_{1}$, ergeben sich Erwartungswert und Varianz von $\widetilde{W}_{1}$ als

$$
\begin{gathered}
E_{0}\left[\widetilde{W}_{1}\right]=W_{0}+\mu X_{0}-2 b_{0} X_{0}^{2}-H_{A}\left(P_{0}+\mu-F_{0,1}\right), \\
\operatorname{Var}_{0}\left[\widetilde{W}_{1}\right]=\left(X_{0}-H_{A}\right)^{2} \sigma^{2}+X_{0}^{4} \eta^{2}-2\left(X_{0}-H_{A}\right) X_{0}^{2} \sigma \eta \rho .
\end{gathered}
$$

Mit diesen Momenten können die FOC geschrieben werden als

$$
\begin{aligned}
& \frac{\partial\left(E_{0}\left[\left(\widetilde{W}_{1}\right)\right]-\frac{1}{2} A \operatorname{Var}_{0}\left[\left(\widetilde{W}_{1}\right)\right]\right)}{\partial H_{A}} \stackrel{!}{=} 0 \\
\Leftrightarrow & -P_{0}-\mu+F_{0,1}-\frac{1}{2} A\left[-2\left(X_{0}-H_{A}\right) \sigma^{2}+2 X_{0}^{2} \sigma \eta \rho\right]=0, \\
\Leftrightarrow & \frac{\partial\left(E_{0}\left[\left(\widetilde{W}_{1}\right)\right]-\frac{1}{2} A \operatorname{Var}_{0}\left[\left(\widetilde{W}_{1}\right)\right]\right)}{\partial X_{0}} \stackrel{!}{=} 0 \\
& -\frac{1}{2} A\left[2 \left(b_{0} X_{0}\right.\right.
\end{aligned}
$$


Zur Bestimmung des Forwardpreises wird die Gleichung (A.3) nach $\mu$ aufgelöst und in Gleichung (A.2) eingesetzt. Das Auflösen dieser Gleichung nach $F_{0,1}$ in Kombination mit der Markträumungsbedingung ergibt Gleichung (3.26).

Gleichung (A.3) ist ein Polynom dritten Grades mit der eindeutigen (realen) Lösung $X_{0, *}:$

$$
\begin{aligned}
X_{0, *}= & -\frac{1}{6} \frac{-3^{\frac{1}{3}}\left(-\left(N_{1}+M_{1}-\sqrt{3} \sqrt{\frac{N_{2}+M_{2}}{A}}\right) A^{2}\right)^{\frac{2}{3}}+N_{3}+M_{3}}{\eta\left(-\left(N_{1}+M_{1}-\sqrt{3} \sqrt{\frac{N_{2}+M_{2}}{A}}\right) A^{2}\right)^{\frac{1}{3}}} \\
& +\frac{1}{2} \frac{\sigma \rho A\left(-\left(N_{1}+M_{1}-\sqrt{3} \sqrt{\frac{N_{2}+M_{2}}{A}}\right) A^{2}\right)^{\frac{1}{3}}}{\eta\left(-\left(N_{1}+M_{1}-\sqrt{3} \sqrt{\frac{N_{2}+M_{2}}{A}}\right) A^{2}\right)^{\frac{1}{3}}},
\end{aligned}
$$

mit

$$
\begin{aligned}
N_{1} \equiv & 36 \sigma \rho b_{0}+9 \sigma^{3} \rho A-18 \eta m-9 \sigma^{3} \rho^{3} A \\
M_{1} \equiv & \left(18 \sigma^{2} \rho^{2} A \eta-18 \eta A \sigma^{2}\right) H_{*}, \\
N_{2} \equiv & 512 b_{0}^{3}+384 b_{0}^{2} A \sigma^{2}-144 b_{0}^{2} \sigma^{2} \rho^{2} A+96 b_{0} A^{2} \sigma^{4}-72 b_{0} A^{2} \sigma^{4} \rho^{2}-432 A \sigma \rho b_{0} \eta \mu \\
& -9 A^{3} \sigma^{6} \rho^{2}+108 A \eta^{2} \mu^{2}+8 A^{3} \sigma^{6}-108 A^{2} \sigma^{3} \rho \eta \mu+108 A^{2} \eta \mu \sigma^{3} \rho^{3}, \\
& \\
M_{2} \equiv & {\left[768 b_{0}^{2} A \sigma \eta \rho+216 A^{2} \eta^{2} \mu \sigma^{2}-216 A^{2} \sigma^{2} \rho^{2} \eta^{2} \mu+72 A^{3} \sigma^{5} \eta \rho^{3}\right.} \\
& -48 b_{0} A^{2} \sigma^{3} \eta \rho-60 A^{3} \sigma^{5} \eta \rho-144 b_{0} A^{2} \sigma^{3} \eta \rho^{3}-120 A^{3} \sigma^{4} \eta^{2} \rho^{2} H_{*} \\
& -36 A^{3} \sigma^{4} \eta^{2} \rho^{4} H_{*}+108 A^{3} \eta^{2} \sigma^{4} H_{*}+384 b_{0} A^{2} \sigma^{2} \eta^{2} \rho^{2} H_{*} \\
& \left.+64 A^{3} \sigma^{3} \eta^{3} \rho^{3} H_{*}^{2}\right] H_{*}, \\
& \\
N_{3} \equiv & 83^{\frac{2}{3}} A b_{0}+23^{\frac{2}{3}} A^{2} \sigma^{2}-33^{\frac{2}{3}} A^{2} \sigma^{2} \rho^{2}, \\
M_{3} \equiv & 43^{\frac{2}{3}} A^{2} \sigma \eta \rho H_{*} .
\end{aligned}
$$




\section{A.2 Simultane Liquiditätseffekte}

Tabelle A.1: Determinanten der Futuresliquidität: Simultane Effekte

\begin{tabular}{|c|c|c|c|c|}
\hline & \multicolumn{2}{|c|}{$X M L_{F}$} & & \multirow{2}{*}{$G B S_{F}$} \\
\hline & 50,000 Euros & 100,000 Euros & & \\
\hline \multirow{2}{*}{$X L M_{A}$} & $0,249^{* * *}$ & $0,932^{* *}$ & $G B S_{A}$ & $0,816^{* * *}$ \\
\hline & $(0,000)$ & $(0,016)$ & & $(0,004)$ \\
\hline \multirow[t]{2}{*}{ Volatilität } & $0,010 * * *$ & $0,006^{* * *}$ & Volatilität & $0,016^{* * *}$ \\
\hline & $(0,000)$ & $(0,002)$ & & $(0,000)$ \\
\hline \multirow{2}{*}{ LiqRisiko } & $0,001^{* * *}$ & $0,001^{* * *}$ & LiqRisiko $_{G B S}$ & $-0,021$ \\
\hline & $(0,000)$ & $(0,001)$ & & $(0,886)$ \\
\hline \multirow{2}{*}{ Laufzeit } & $0,179 * * *$ & $0,153^{* * *}$ & Laufzeit & $0,104^{* * *}$ \\
\hline & $(0,000)$ & $(0,000)$ & & $(0,000)$ \\
\hline \multirow[t]{2}{*}{ Dividende (10 Tage) } & 0,112 & 0,075 & Dividende (10 Tage) & $0,248^{* * *}$ \\
\hline & $(0,157)$ & $(0,360)$ & & $(0,001)$ \\
\hline \multirow[t]{2}{*}{$L I B O R$} & $-0,377^{* * *}$ & $-0,214^{* * *}$ & $L I B O R$ & $0,870^{* * *}$ \\
\hline & $(0,001)$ & $(0,005)$ & & $(0,000)$ \\
\hline \multirow[t]{2}{*}{ Konstante } & $1,012^{* * *}$ & $0,921^{* * *}$ & Konstante & $-0,144$ \\
\hline & $(0,000)$ & $(0,000)$ & & $(0,294)$ \\
\hline \multirow{2}{*}{$\begin{array}{l}\text { Beobachtungen } \\
\text { within }-R^{2}\end{array}$} & 18.860 & 15.189 & & 21.988 \\
\hline & 0,0357 & 0,0727 & & 0,0806 \\
\hline
\end{tabular}

Die Tabelle zeigt die Ergebnisse der Fixed-Effects Panelregressionen mit der Liquidität des Futuresmarktes als abhängiger Variable. Die abhängige Variable der ersten beiden Regressionen ist das Liquiditätsmaß $X L M_{F}$ für ein Volumen von 50.000 Euro und 100.000 Euro. Die erklärenden Variablen sind $X L M_{S}$, Volatilität, LiqRisiko, Laufzeit, Dividende (10 Tage) und $L I B O R$. Die abhängige Variable der dritten Regression ist die Geld-Brief-Spanne $G B S_{F}$. Die erklärenden Variablen sind $G B S_{A}$, Volatilität, LiqRisiko ${ }_{G B S}$, Laufzeit, Dividende (10 Tage) und LIBOR. Der als Konstante ausgewiesene Wert ist der mean fixed effect. Die p-Werte sind in Klammern angegeben und wurden bestimmt durch geclusterte Standardfehler. Der Beobachtungszeitraum ist vom 01.01.2010 bis zum 31.12.2011. ***, **, und * geben die statistische Signifikanz auf dem $1 \%, 5 \%$, and $10 \%$ Niveau an. 


\section{Versicherung an Eides Statt}

Ich versichere an Eides Statt, dass ich die eingereichte Dissertation "Liquiditätszusammenhänge zwischen Kassa- und Derivatemärkten" selbstständig verfasst habe. Anderer als der von mir angegebenen Hilfsmittel und Schriften habe ich mich nicht bedient. Alle wörtlich oder sinngemäß den Schriften anderer Autorinnen und/ oder Autoren entnommenen Stellen habe ich kenntlich gemacht.

Ort, Datum

Paolo Krischak 